\title{
Bending and Shear Behavior of Pultruded Glass Fiber Reinforced Polymer Composite Beams With Closed and Open Sections
}

\author{
Daniel Douglas Estep \\ West Virginia University
}

Follow this and additional works at: https://researchrepository.wvu.edu/etd

\author{
Recommended Citation \\ Estep, Daniel Douglas, "Bending and Shear Behavior of Pultruded Glass Fiber Reinforced Polymer \\ Composite Beams With Closed and Open Sections" (2014). Graduate Theses, Dissertations, and Problem \\ Reports. 545. \\ https://researchrepository.wvu.edu/etd/545
}

This Thesis is protected by copyright and/or related rights. It has been brought to you by the The Research Repository @ WVU with permission from the rights-holder(s). You are free to use this Thesis in any way that is permitted by the copyright and related rights legislation that applies to your use. For other uses you must obtain permission from the rights-holder(s) directly, unless additional rights are indicated by a Creative Commons license in the record and/ or on the work itself. This Thesis has been accepted for inclusion in WVU Graduate Theses, Dissertations, and Problem Reports collection by an authorized administrator of The Research Repository @ WVU. For more information, please contact researchrepository@mail.wvu.edu. 


\title{
Bending and Shear Behavior of Pultruded Glass Fiber Reinforced Polymer Composite Beams With Closed and Open Sections
}

\author{
Daniel Douglas Estep
}

Thesis submitted to the

Benjamin M. Statler College of Engineering and Mineral Resource at West Virginia University in partial fulfillment of the requirements

for the degree of

\section{Master of Science \\ in \\ Civil Engineering}

Approved by

Hota V. S. GangaRao, Ph.D., Chair

Udaya B. Halabe, Ph.D.

P. V. Vijay, Ph.D.

\section{Department of Civil and Environmental Engineering}

\author{
Morgantown, West Virginia \\ 2014
}

Keywords: bending, shear, box beam, channel, wide flange, pultruded, glass fiber reinforced polymer composites, lateral torsional buckling 


\title{
ABSTRACT \\ Bending and Shear Behavior of Pultruded Glass Fiber Reinforced Polymer Composite Beams With Closed and Open Sections
}

\author{
Daniel Douglas Estep \\ Constructed Facilities Center, West Virginia University
}

Several advantages, such as high strength-to-weight ratio, high stiffness, superior corrosion resistance, and high fatigue and impact resistance, among others, make FRPs an attractive alternative to conventional construction materials for use in developing new structures as well as rehabilitating in-service infrastructure. As the number of infrastructure applications using FRPs grows, the need for the development of a uniform Load and Resistance Factor Design (LRFD) approach, including design procedures and examples, has become paramount.

Step-by-step design procedures and easy-to-use design formulas are necessary to assure the quality and safety of FRP structural systems by reducing the possibility of design and construction errors. Since 2008, the American Society of Civil Engineers (ASCE), in coordination with the American Composites Manufacturers Association (ACMA), has overseen the development of the Pre-Standard for Load and Resistance Factor Design (LRFD) of Pultruded Fiber Reinforced Polymer (FRP) Structures using probability-based limit states design. The fifth chapter of the pre-standard focuses on the design of members in flexure and shear under different failure modes, where the current failure load prediction models proposed within have been shown to be highly inaccurate based on experimental data and evaluation performed by researchers at the West Virginia University Constructed Facilities Center.

A new prediction model for determining the critical flexural load capacity of pultruded GFRP square and rectangular box beams is presented within. This model shows that the type of failure can be related to threshold values of the beam span-to-depth ratio $(L / h)$ and total flange width-to-thickness ratio $\left(b_{f} / t\right)$, resulting in three governing modes of failure: local buckling failure in the compression flange $(4 \leq L / h<6)$, combined strain failure at the web-flange junction $(6 \leq L / h \leq 10)$, and bending failure in the tension flange $(10<L / h \leq 42)$. Broadly, the proposed equations are predicting critical flexural load capacities within $\pm 22.3 \%$ of experimental data for all cases, with over $70 \%$ of all experimental data with within $\pm 10 \%$ error.

A second prediction model was developed for predicting the critical lateral-torsional buckling (LTB) load for pultruded GFRP open sections, including wide flange (WF) sections and channels. Multiple LTB equations from several sources were considered and applied but yielded inaccurate results, leading to the development of this new critical buckling load prediction model based on the well-established elastic LTB strength equation for steel. By making a series of modifications to equations for calculating the weak axis moment of inertia, torsional warping constant, and torsion constant for open sections, as well as recognizing the influence of the shear lag phenomenon, the critical LTB load is predicted within $\pm 15.2 \%$ of experimental data for all channel and WF specimens tested and evaluated in the study. 


\section{ACKNOWLEDGEMENTS}

I would first like to thank my advisor, Dr. Hota GangaRao, for providing me the opportunity to pursue a Master of Science degree, for his guidance throughout the completion of my projects, and for serving as the chair of my graduate advisory committee. His valuable experience and knowledge have made the last year and a half a tremendous learning experience. I thank him for his willingness to discuss any and all plans and challenges I faced throughout my post-baccalaureate work.

I would also like to thank Dr. Udaya Halabe for agreeing to serve on my graduate advisory committee, as well as for his advice throughout my graduate schooling. Additionally, I would like to thank Dr. P. V. Vijay for agreeing to serve on my graduate advisory committee, and providing valuable suggestions as needed.

Furthermore, I would like to extend a special thanks to Jerry Nestor and Brian Walker for the countless hours they spent helping me complete my experimental work in the lab, Mark Skidmore for his instruction on how to operate the lab equipment and properly install fixtures for testing, and Dr. David Dittenber for his advice and aid in showing me what it takes to be a successful graduate student.

Most importantly, I would like to thank my family for their constant support throughout the graduate school process and for their reassuring words during my most difficult challenges. Without their encouragement, none of this would be possible.

Lastly, I would like to thank Bedford Reinforced Plastics and Creative Pultrusions, Inc., both located in Pennsylvania, USA, for their generosity in funding and donating a wide variety of pultruded GFRP samples for use in the testing completed for this study, and also the National Science Foundation for partial funding of this research. 


\section{TABLE OF CONTENTS}

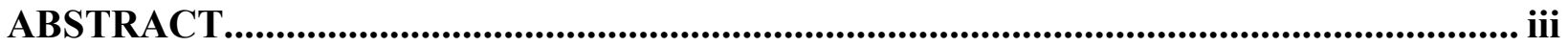

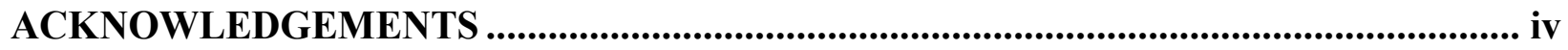

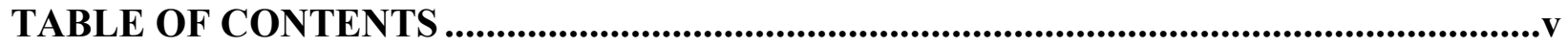

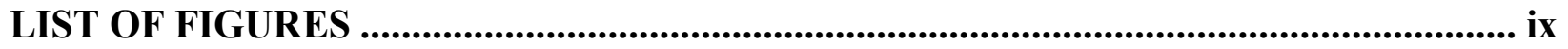

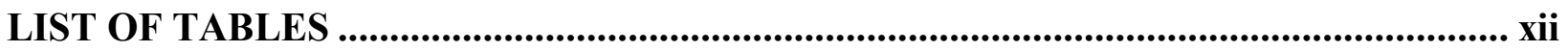

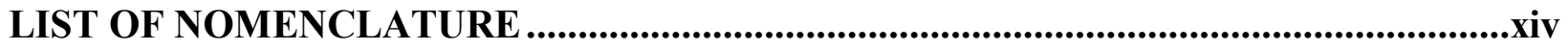

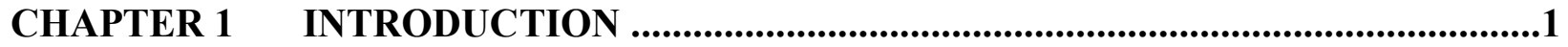

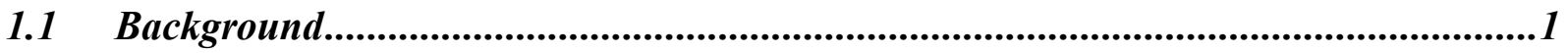

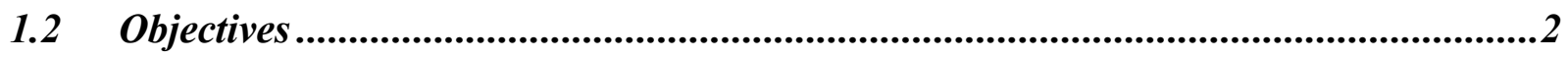

1.3 Scope and Organization of Thesis...............................................................................3

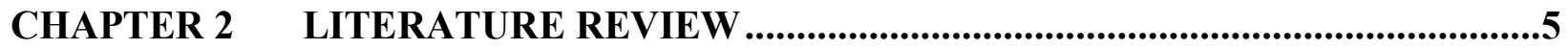

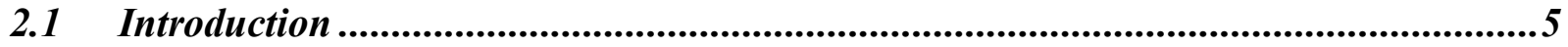

2.2 Flexural and Shear Moduli Determination..............................................................6

2.3 Bending Behavior of GFRP Box Beams...............................................................12

$2.4 \quad$ LTB of GFRP Open Sections ...............................................................................29

CHAPTER $3 \quad$ BOX BEAM FAILURE PREDICTION ................................................51

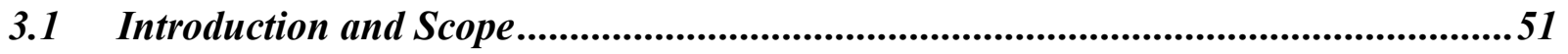

3.2 Samples and Test Procedure .........................................................................52

3.2.1 Test Procedure for Spans Less than 20 inches ..........................................54 
3.2.2 Test Procedure for Spans Ranging from 20 inches to 30 inches......

3.2.3 Test Procedure for Spans Greater than 30 inches

3.3 Governing Modes of Failure ....................................................................60

3.3.1 Local Buckling Failure in the Compression Flange .......................................60

3.3.2 Principal Strain Failure in Tension Zone of the Web ......................................66

3.3.3 Bending Failure in the Tension Flange .................................................... 72

$3.4 \quad$ Stress Intensity Factor ............................................................................ 75

3.5 Existing Critical Flexural Capacity Prediction Models.........................................78

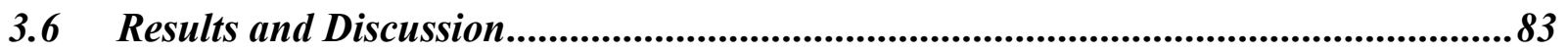

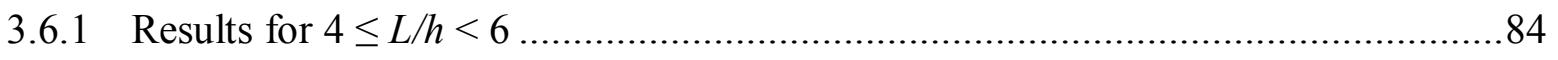

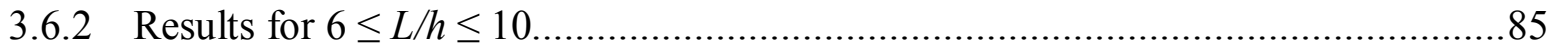

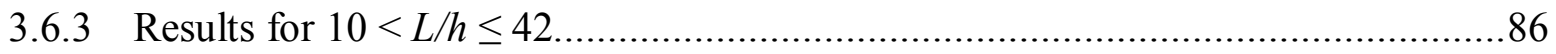

3.7 Results from Existing Prediction Models .................................................................89

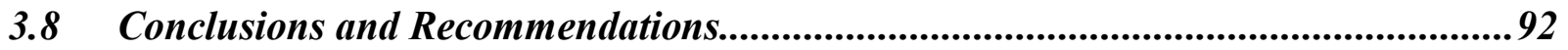

CHAPTER 4 LATERAL-TORSIONAL BUCKLING LOAD PREDICTION FOR

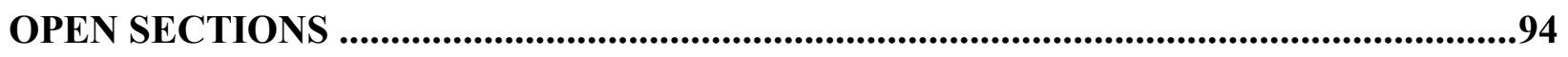

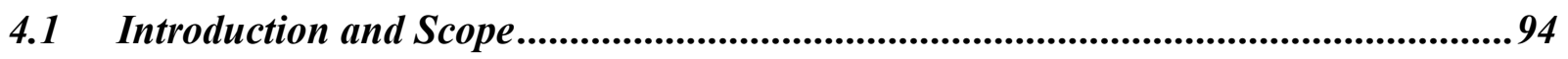

4.2 Samples and Test Procedure ..................................................................................95

4.3 Experimental Data Analysis............................................................................... 101

4.4 Critical LTB Load Prediction Model............................................................ 104 


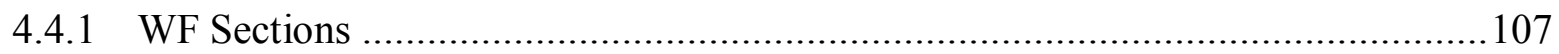

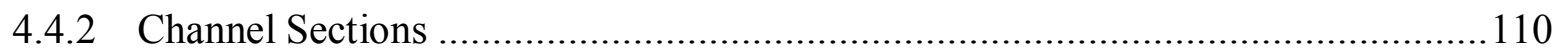

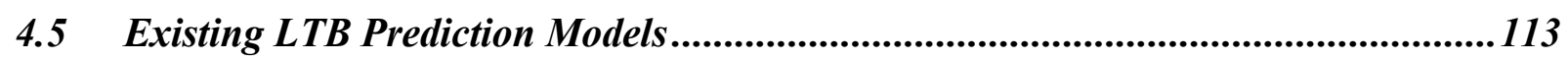

4.6 Results of Proposed LTB Prediction Model ..................................................................117

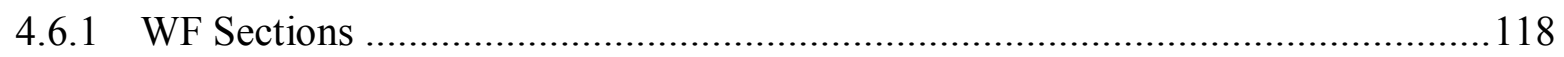

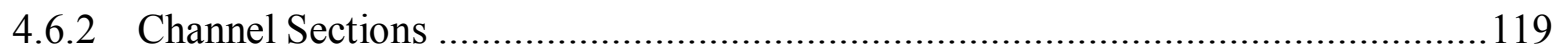

$4.7 \quad$ Results of Existing LTB Prediction Models .................................................................120

4.7.1 ASCE/ACMA LRFD Draft Pre-Standard Equation Results .................................120

4.7.2 Structural Plastics Design Manual Equation Results............................................121

$4.8 \quad$ Summary, Conclusions, and Recommendations .......................................................... 123

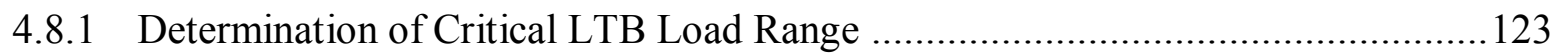

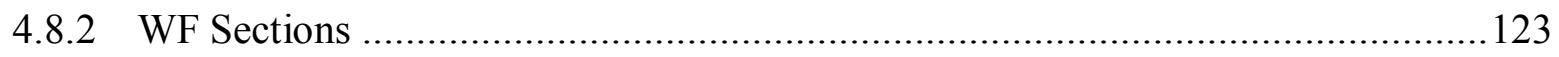

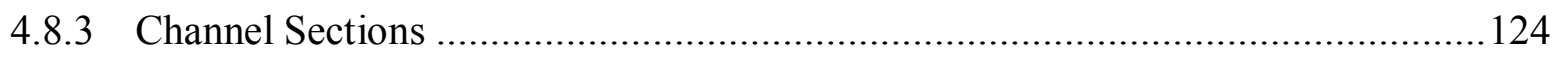

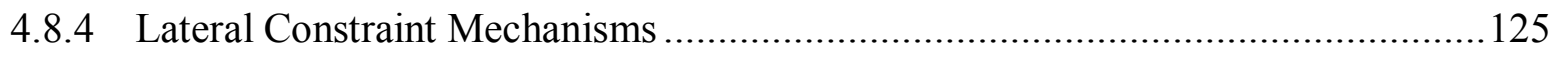

CHAPTER 5 CONCLUSIONS AND RECOMMENDATIONS.......................................127

5.1 Critical Flexural Capacity of GFRP Box Sections.....................................................128

5.2 Critical LTB Capacity of GFRP Open Sections ..........................................................130

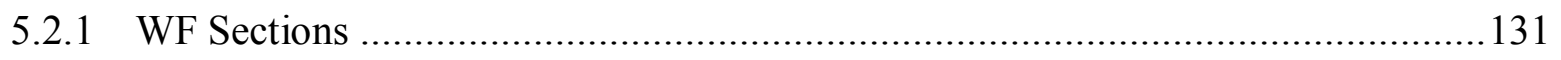

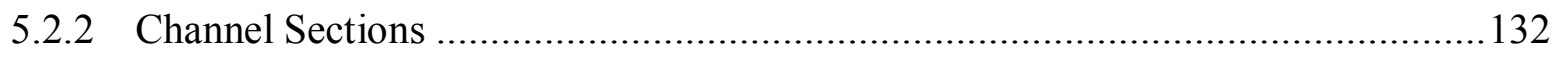

5.2.3 Analysis of Lateral Constraint Mechanisms ……..............................................133 
REFERENCES.

APPENDIX A - SAMPLE CALCULATION OF PRINCIPAL STRAINS.

APPENDIX B - DERIVATION OF STRESS INTENSITY FACTOR .144

APPENDIX C - COUPON LEVEL TENSION TEST RESULTS ..................................148 APPENDIX D - PLOTS USED FOR DETERMINATION OF CRITICAL LTB LOAD RANGE. .156 


\section{LIST OF FIGURES}

Figure 2-1 Beam at end of first stiffening phase (Palmer et al. 1998) ................................25

Figure 2-2 Tearing damage visible in the tension flange (Palmer et al. 1998) .......................26

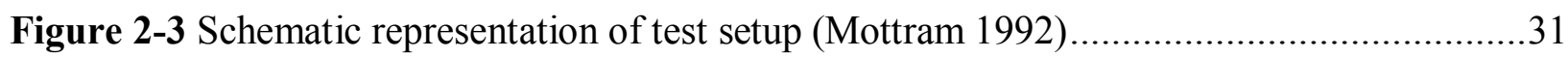

Figure 2-4 Critical loading for lateral-torsional buckling of simply-supported I-beams (Mottram 1992) .36

Figure 2-5 Critical load ratio $P_{o} / P_{L}$ versus ratio $l / r$ for load applied at the centroid 40

Figure 2-6 Critical load ratio $P_{o} / P_{L}$ versus ratio $b_{w} / b_{f}$ for load applied at the centroid generated by (a) using a fixed web depth $b_{w}$ and (b) using a fixed width $b_{f}$ (Barbero and Raftoyiannis 1994) .40

Figure 2-7 Diagram of loading disc system (a) prior to LTB failure and (b) after LTB failure ..48

Figure 3-1 Dimensions for use in proposed model for square and rectangular box sections .......52 Figure 3-2 Setup using Instron System 8500 Series and GFRP load distributing plate for testing of a $3 " \times 3 " \times 0.25 "$ sample at a test span of 18 inches. .56

Figure 3-3 Setup using Instron Industrial Series 1000HDX Model and steel load distributing plate for testing of a $3^{\prime \prime} \times 3 " \times 0.25^{\prime \prime}$ sample at a test span of 24 inches.

Figure 3-4 Setup using manually operated hydraulic actuator and steel load distributing spacer column for testing of a $3 " \times 3 " \times 0.25 "$ sample at a test span of 72 inches.

Figure 3-5 Web crushing caused by local instability in the compression flange 61

Figure 3-6 Percentage of shear influence on total deflection for specimen having $b_{c}=0.8 b_{f}$ and

$d_{w}=0.9 h$ 
Figure 3-7 Comparison of predicted flexural load capacity versus experimental data for local buckling in the compression flange failure mode (a) before SIF is applied and (b) after SIF is applied

Figure 3-8 Crack formation visible in the tension zone of the web due to principal strain failure

Figure 3-9 Comparison of predicted flexural load capacity versus experimental data for principal strain failure in the tension zone of the web (a) before SIF is applied and (b) after SIF is applied

Figure 3-10 Comparison of predicted flexural load capacity versus experimental data for bending failure in the tension flange (a) before SIF is applied and (b) after SIF is applied........74

Figure 3-11 Classic bending failure in the tension flange of a $6 " \times 4 " \times 0.25$ " box specimen (Qureshi 2012) .75

Figure 3-12 Predicted load vs. experimental load based on proposed flexural load capacity prediction model .84

Figure 4-1 Dimensions of (a) channel section and (b) WF section used in the proposed model 95

Figure 4-2 Approximate locations of shear center and center of gravity for WF and channel sections. .97

Figure 4-3 (a) Steel angle attached to the web of a $8 " \times 2.25^{\prime \prime} \times 0.375^{\prime \prime}$ channel using Pliogrip adhesive and (b) 1 kip load cell used to capture horizontal resistance provided by lateral bracing

Figure 4-4 Front view of test setup for $6 " \times 1.625 " \times 0.1875$ " channel section subjected to threepoint bending with intermediate lateral brace points $2.5 \mathrm{ft}$. from midspan. 100 
Figure 4-5 Rear view of test setup for $14 " \times 3.5 " \times 0.75$ " channel section subjected to three-point bending with intermediate lateral brace points $2.5 \mathrm{ft}$. from midspan 101

Figure 4-6 (a) and (b) Selection of lower and upper bounds of critical LTB load range by visual inspection. 103

Figure 4-7 Load vs. longitudinal strain in the compression flange at varying distances from the web for a $8 " \times 8^{\prime \prime} \times 0.375^{\prime \prime}$ WF section tested at a span of 108 in. .109 


\section{LIST OF TABLES}

Table 2-1 Comparison for simplified design analysis for ultimate load (modified from Mottram 1991) 18

Table 2-2 Elastic constants of composite material used in simulations (Palmer et al. 1998)......27

Table 2-3 Section properties and critical load values for I102/51/6 beams at $1500 \mathrm{~mm}$ span (modified from Mottram 1992). .34

Table 2-4 Theoretical predictions of critical loading for pultruded profile I102/51/6 at a span of $1500 \mathrm{~mm}$ (modified from Mottram 1992). .37

Table 3-1 Summary of samples tested for analysis of square and rectangular box sections ........52

Table 3-2 Laminate properties for box sections included in this study based on coupon level tension testing .53

Table 3-3 Flexural load capacity prediction results for box members where $4 \leq L / h<6$ using Equation (3-1) .85

Table 3-4 Flexural load capacity prediction results for box members where $6 \leq L / h \leq 10$ using Equation (3-8) .86

Table 3-5 Flexural load capacity prediction results for box members where $10<L / h<42$ using Equation (3-10) .88

Table 3-6 Results for critical flexural capacity prediction model from Nov. 2010 draft of LRFD

Pre-Standard .90

Table 3-7 Results for critical flexural capacity prediction model from May 2013 draft of LRFD

Pre-Standard .91

Table 4-1 Cross-sectional dimensions of samples tested for analysis of WF sections 96

Table 4-2 Cross-sectional dimensions of samples tested for analysis of channel sections .96 
Table 4-3 Laminate properties for WF sections included in this study from tension coupon testing

Table 4-4 Laminate properties for channel sections included in this study from tension coupon testing .97

Table 4-5 Comparison of $b_{f}$ and $b_{e f f}$ for channel specimens 113

Table 4-6 Critical LTB load prediction for WF sections using the proposed model 119

Table 4-7 Critical LTB load prediction for channel sections using proposed model 120

Table 4-8 Critical LTB load prediction for WF sections using the ASCE/ACMA equation ..... 121 Table 4-9 Critical LTB load prediction for channel sections using the ASCE/ACMA equation

Table 4-10 Critical LTB load prediction for WF sections using ASCE Plastics Design Manual equation 122

Table 4-11 Critical LTB load prediction for channel sections using ASCE Plastics Design Manual equation. 122 


\section{LIST OF NOMENCLATURE}

\section{Variables}

A total area of cross-section, in. ${ }^{2}$

$b_{c} \quad$ clear width of the flange, in.

$b_{\text {eff }}$ effective flange width, in.

$b_{f} \quad$ full width of the flange, in.

c distance from neutral axis to the outside tension or compression fiber of the beam, in.

$C_{1} \quad$ lateral buckling coefficient

$C_{w} \quad$ torsional warping constant, in. ${ }^{6}$

$C_{b} \quad$ modification factor for non-uniform bending moment variation for a beam segment laterally unbraced except at the segment ends

$d \quad$ overall depth of section, in. $=h-t_{f}$

$d_{w} \quad$ clear depth of web, in. $=h-2 t_{f}$

$D_{J} \quad$ torsional rigidity, kip $\cdot$ in. $^{2}$

E modulus of elasticity, psi

$E_{L} \quad$ characteristic value of the longitudinal modulus, psi

$E_{L, f} \quad$ characteristic longitudinal modulus of the flange, psi

$E_{L, w} \quad$ characteristic longitudinal modulus of the web, psi

$E_{T, f} \quad$ characteristic transverse modulus of the flange, psi

$E_{T, w} \quad$ characteristic transverse modulus of the web, psi

$f_{c r} \quad$ critical buckling stress, psi

$F_{L} \quad$ characteristic longitudinal strength of the member, psi

$F_{L f} \quad$ characteristic longitudinal strength in the flange, psi

$F_{L w} \quad$ characteristic longitudinal strength in the web, psi 
G $\quad$ shear modulus, psi

$G_{L T} \quad$ characteristic value of the in-plane shear modulus, psi

$G_{L T, f}$ characteristic value of the in-plane shear modulus of the flange, psi

$h \quad$ total height of section, in.

I moment of inertia of the member about the axis of bending, in. ${ }^{4}$

If moment of inertia of the flanges about the axis of bending, in. ${ }^{4}$

$I_{w} \quad$ moment of inertia of the webs about the axis of bending, in. ${ }^{4}$

$I_{X} \quad$ strong-axis moment of inertia, in. ${ }^{4}$

$I_{y}$ weak-axis moment of inertia of one flange, in. ${ }^{4}$

J torsion constant, in. ${ }^{4}$

$k \quad$ corner stress concentration factor

$k_{f} \quad$ shear lag effect factor

$k_{r} \quad$ rotational spring constant, $\mathrm{lbf} / \mathrm{rad}$

$K \quad$ effective length factor

$L \quad$ total span length, in.

$L_{b} \quad$ distance between lateral bracing points, in.

Mo applied moment, lbf $\cdot$ in.

$M_{c r} \quad$ elastic lateral-torsional buckling strength, $\mathrm{lbf} \cdot \mathrm{in}$.

$M_{n} \quad$ nominal strength of members due to lateral-torsional buckling, lbf.in.

$M_{x c} \quad$ critical buckling moment, lbf $\cdot$ in.

$P \quad$ applied load, lbf

$P_{c r} \quad$ critical flexural load capacity, lbf

$P_{e 2} \quad$ Euler column load for buckling in the weak direction, lbf

$r \quad$ average radius of the reentrant corner, in. 
$r_{i} \quad$ inner radius of the reentrant corner, in.

$S_{t} \quad$ transformed section modulus about the axis of bending, in. ${ }^{3}$

$S_{1} \quad$ strong-axis section modulus, in. ${ }^{3}$

SIF stress intensity factor

$t \quad$ wall thickness, in.

$t_{f} \quad$ thickness of flange, in.

$t_{w} \quad$ thickness of web, in.

$u \quad$ displacement of centroid in x-direction, in.

$v \quad$ displacement of centroid in y-direction, in.

$y$ distance from the neutral axis to the extreme fiber of the member, in.

$y_{f}$ distance from the neutral axis to the extreme fiber of the flange, in.

$y_{w}$ distance from the neutral axis to the extreme fiber of the web, in.

$\alpha \quad$ shape factor

$\beta \quad$ constant related to stress concentration factor

$\gamma_{\max }$ mean value of maximum shear strain at failure

$\gamma_{x y} \quad$ shear strain

$\delta \quad$ deflection, in.

$\varepsilon_{X X} \quad$ transverse strain

$\varepsilon_{y y} \quad$ longitudinal strain

$v_{L T} \quad$ characteristic value of Poisson's ratio

$\xi \quad$ coefficient of restraint

$\sigma \quad$ bending stress at outer fiber of cross section, psi

$\sigma_{x c} \quad$ critical flexural buckling stress, psi

$\varphi \quad$ angle of twist, radians 


\section{CHAPTER 1 INTRODUCTION}

\subsection{Background}

Fiber reinforced polymer (FRP) composites exhibit a multitude of beneficial properties which make them advantageous in a variety of applications in comparison to traditional construction materials, such as concrete and steel. These properties include: high strength-toweight ratio, high stiffness, high fatigue and impact resistance, superior corrosion resistance, low thermal and electrical conductivity, ease of fabrication, low cost of installation, and low lifecycle cost (Liang and GangaRao 2013). As FRPs gain wider acceptance for use in the development and rehabilitation of infrastructure, the need to develop Load and Resistance Factor Design (LRFD) specifications, including design approaches and examples for FRP composites, has become paramount.

Since 2008, the American Society of Civil Engineers (ASCE), in coordination with the American Composites Manufacturers Association (ACMA), has overseen the development of the Pre-Standard for Load and Resistance Factor Design (LRFD) of Pultruded Fiber Reinforced Polymer (FRP) Structures using probability-based limit states design. The fifth chapter of the pre-standard focuses on the design of members in flexure and shear under different failure modes. Through extensive research performed at the West Virginia University Constructed Facilities Center (WVU-CFC), the equations provided in the most recent draft version of the prestandard have been shown to be highly inaccurate for both closed and open sections.

The development of a more accurate critical load prediction model based on easy-to-use design formulas is necessary to assure the quality and safety of FRP structural systems by reducing the possibility of design and construction errors. Previous models proposed by the 
ASCE/ACMA pre-standard involve the application of multiple design formulas, taking the lowest calculated critical load to be the design load, which significantly increases the chance of design errors. Through extensive experimental work, it can be shown that the failure mode can be characterized based on threshold values related to certain ratios of the span length and crosssectional dimensions. This allows the designer to apply one simple equation to obtain the critical load needed for the design of a flexural member within a structural system.

\subsection{Objectives}

The objectives of this study are:

- To perform experimental evaluation of GFRP square and rectangular box members and analyze the associated critical flexural load capacity of each section.

- To perform experimental evaluation of GFRP wide flange (WF) and channel sections and analyze the acquired data in order to identify the critical lateraltorsional buckling (LTB) load of each section.

- To analyze the accuracy and consistency of existing prediction models for both the critical flexural load capacity of closed GFRP sections, and for the critical LTB load capacity of open GFRP sections.

- To develop more accurate and easy to compute prediction models for both the critical flexural load capacity of closed GFRP sections, as well as the critical LTB load of open GFRP sections. 


\subsection{Scope and Organization of Thesis}

Chapter 2 provides a literature review of published books and journal articles related to the experimental objectives of this study. Previous studies related to the flexural load capacity of closed and open GFRP sections are discussed, as well as brief discussion related to theories of elastic stability and the mechanics behind lateral-torsional buckling. A short review and comparison of full-scale and coupon-level methods for determining orthotropic elastic material properties is also provided.

Chapter 3 details the experimental testing and subsequent analysis of data for GFRP square and rectangular box sections. The testing procedure is covered in detail, and flexural load capacity of each test specimen is reported. The proposed critical flexural load capacity prediction model based on three governing modes of failure is presented, with derivations shown for the developed equations. Equations from two existing critical flexural load capacity models are explained and analyzed. Both models are compared with experimental flexural load capacity data from three-point bend testing of square and rectangular box sections with depths and widths ranging from 3 inches to 6 inches, and wall thicknesses ranging from $1 / 4$ inch to $3 / 8$ inch. Test spans ranged from 18 inches to 180 inches. The results from the proposed model and existing models compared to experimental data are shown and discussed.

Chapter 4 details the experimental testing and evaluation of GFRP channel and WF sections, and the analytical procedure used to develop the critical LTB load range of each specimen size. A critical LTB load prediction model, developed through a series of modifications to the elastic LTB strength equation for steel sections is presented in detail. Two existing equations for predicting the critical LTB load of FRP sections are explained and analyzed. These models are compared with experimental critical LTB load data from specimens 
ranging from 6 inches to 12 inches in depth for WF sections and from 4 inches to 14 inches in depth for channel specimens. WF section flange widths ranged from 4 inches to 12 inches, and channel legs ranged from 1.125 inches to 3.5 inches. Wall thicknesses ranged from 0.375 to 0.5 inches for WF sections, and from 0.1875 inches to 0.75 inches for channel specimens. The results from the proposed model and the existing equations compared to the experimental critical LTB load data are shown and discussed.

Chapter 5 provides a summary of the results presented within Chapters 3 and 4. Conclusions previously stated within those chapters are expanded on, with detailed recommendations provided for the future improvement of the model proposed within. 


\section{CHAPTER 2 LITERATURE REVIEW}

\subsection{Introduction}

As previously mentioned, the use of fiber-reinforced polymer composites for infrastructural applications is growing rapidly. One major hindrance, however, to the growth of FRPs is a better understanding of the thermo-mechanical behavior of these complex materials. This lack of fundamental understanding of the behavior makes the development of simple analytical and design procedures extremely difficult. Various design codes for pultruded FRP sections have been developed in the past on a theoretical basis with very little experimental verification. These codes have a tendency to be mostly conservative in nature, and even inconsistent and unsafe at times.

Specific attempts have been made to understand the overall behavior, including local and global stability of pultruded structural components. Numerical procedures, such as finite element (FE) modelling are widely considered to be accurate analytical tools for pultruded composite structures. With proper modelling including realistic boundary conditions and precise orthotropic material properties, finite element models can predict failure modes and calculate stresses with a high degree of accuracy. However, FE analysis is time consuming and requires in-depth training of technical personnel, and therefore is not suitable for design purposes, particularly in the preliminary phases. In spite of these sorts of technical hurdles, research in the area of simplistic theoretical design equations for pultruded FRP members remains limited.

To advance the development of simple (design) equations for polymer composite member behavior, limited research has been performed to better understand the strength and stiffness to failure of pultruded members. Despite incremental advances, there still exists a large amount of 
disagreement within the composite community in regards to calculating material and section properties. While coupon-level testing can provide a low cost solution for obtaining material properties, results of this testing do not always correlate well with full-scale test results, especially in establishing failure modes. Furthermore, the results of a coupon tested in tension will not offer the same results as a coupon tested in flexure, and certainly not the same when compared with data from full-scale component response.

To overcome some of the aforementioned difficulties in terms of design of FRP composite members and to gain better understanding based on current research data, a review of previous work related to these issues, with emphasis on flexural behavior including failure modes and lateral-torsional buckling is discussed herein. The organization of this chapter is as follows:

- Section 2.2 provides an overview of some of the various techniques used to measure material and section properties of pultruded FRP composite structural members and their limitations.

- Section 2.3 discusses previous prediction models, both numerical and analytical, for predicting the critical flexural load capacity of pultruded FRP box members.

- Section 2.4 evaluates the lateral-torsional buckling (LTB) behavior of pultruded FRP open sections, including prediction of the critical LTB load capacity.

\subsection{Flexural and Shear Moduli Determination}

Accurate characterization of strength and stiffness properties is essential for successful development and application of any closed-form design models for orthotropic pultruded FRP beams. While most manufacturers will provide nominal design data for their products, these

values tend to be highly conservative and at times unsafe. In order to truly capture the member 
behavior, including their properties, testing must be performed on full-size specimens and also at coupon-levels. Values obtained through tensile coupon testing will not yield the same results as coupons tested under flexure (Timoshenko and Gere 1961). Likewise, the resulting properties obtained from full-scale evaluation will also be different. Some reasons for this variation, as well as a brief analysis of each method are provided in this section. The focus of coupon-level testing will be on tensile test methods because of its applicability to this study.

The most significant advantage to coupon (cut from a component) testing is cost and the ability to harvest many coupons from a single member. Another advantage is the ability to determine the material properties in the individual components (flange or web) of a member where properties are likely to differ (Roberts and Masri 2003). This is particularly useful when the most likely mode of failure is anticipated, and the critical component of a member can be identified, such as the case of lateral-torsional buckling failures. There is some inherent inaccuracy involved with coupon-level testing, particularly in inhomogeneous materials such as pultruded FRPs, where properties may vary somewhat throughout the span length due to microscopic defects and manufacturing inconsistencies.

A major danger with tension testing for material properties stems from the effect of nonuniform ply thickness or spacing. When the thickness of the plies or the spacing between fabric layers is non-symmetric throughout the cross section of the coupon sample, the neutral axis no longer lies at mid-plane. As a tensile load is applied to the specimen, this non-uniformity leads to extensional-bending coupling, resulting in the introduction of both tensile and bending stresses in the sample, and therefore causing a non-uniform strain distribution (Zweben et al. 1979). This means that the maximum strain measured will be higher on one surface compared to the other. Therefore, by using a strain gage to calculate the elastic modulus, the resulting value on one side 
of the coupon will differ from the results on the opposite side, and thus be either higher or lower than the actual value. This source of error can be partially eliminated by installing gages on both sides of the sample, and taking the average of the two results (Zweben et al. 1979). Zweben et al. (1979) showed that an eccentricity, which is the measured distance between the centroid and the neutral axis, of only 2 percent could result in \pm 12 percent error in determining the tensile stiffness property.

Another source of error can be related to the thickness of the resin surface layer of a laminated composite coupon sample. The tensile modulus of the fiber in pultruded FRPs is significantly greater than that of the resin, to the point that the contribution of the resin can be neglected in approximating the tensile modulus of the material. However, because the resin layer is considered in calculating the area of the cross-section of the specimen, its effect on the computed tensile modulus can be significant (Zweben et al. 1979). A study performed by Zweben et al. (1979) proves that the effect of the thickness of the resin layers is less dramatic for tensile coupon testing compared to flexural testing, which can be very sensitive to changes in resin layer thickness. By this reasoning, it can be argued that tensile coupon testing offers greater reliability for use with composite materials and particularly for those manufactured using a hand layup method where the thickness is more likely to be inconsistent throughout the sample (Zweben et al. 1979).

Three-point flexure testing of full-scale beam specimens is another popular method for determining material properties. This type of testing is used in ASTM D790, the standard test method for calculating flexural properties of reinforced polymers. An accurate test for determining the elastic properties of a material should result in a uniform stress component across the gage section (Zweben et al. 1979). This criteria can be used to explain why values 
from tensile coupon testing have a tendency to differ from flexural moduli calculated using three-point bending. If you consider Euler-Bernoulli Beam Theory under pure bending moment of a simply-supported beam, it can be shown that there is a linear stress variation from compression in the upper face to tension in the bottom face of the specimen. The resulting shear stress is a parabolic distribution which is equal to zero at the top and bottom extreme fibers and reaches a maximum at the neutral axis, which is only valid for small deflections (Timoshenko 1962). Another assumption of this model is that the material is both homogeneous and isotropic, of which pultruded members are neither. Therefore, the neutral axis is not likely to coincide with the centroidal axis. Due to this eccentricity, the maximum tensile and compressive stress across the beam will not be equal. In theory, if these were identical, then the tensile and compressive moduli would be equal to the flexural modulus; this, however, is not often the case (Zweben et al. 1979). Nonetheless, we assume on average that the neutral axis coincides with the center of gravity, and the tensile and compressive moduli are equal.

Another major source of error in ASTM D790 is the failure to account for shear deformation. A study by Zweben et al. (1979) revealed that in order to obtain accurate values for the flexural modulus, a span-to-depth ratio of 60 must be used so that shear deformation is negligible. However, recent studies have shown that a span-to-depth ratio around 40 is adequate to neglect shear effects, and this ratio exceeding 50 results in geometrically non-linear response. In order to counteract this issue, simultaneous calculations of the flexural modulus and shear modulus of a full section accounting for shear deformation using Timoshenko Beam Theory were used (Bank 1989) where the deflection under static loading conditions is given by Equations (2-1) and (2-2). 


$$
\begin{gathered}
E_{b} I \frac{\delta \varphi}{\delta z}=M \\
\frac{\delta y}{\delta z}+\varphi=\frac{Q}{A G_{b}}
\end{gathered}
$$

In which

$E_{b}=$ Young's modulus

$I=$ second moment of area

$\varphi=$ bending slope

$Z=$ axial coordinate

$M=$ bending moment

$y=$ beam deflection

$Q=$ shear force

$A=$ cross-sectional area

$G_{b}=$ shear modulus

Here, the subscript $b$ denotes the property of a full-scale beam element. The equation for the shear modulus, $G_{b}$, is given by Equation (2-3) where the shear coefficient, $k$, is dependent on the shape and material properties of the section (Bank 1989).

$$
G_{b}=k G_{\text {material }}
$$

It should be noted that the shear coefficient is depended only on the relative dimensions of the cross-section. Therefore, in theory, all sections of the same relative dimensions, constructed from the same material and possessing the same microstructure would have identical values for the shear modulus (Bank 1987); but, expecting identical values is a bit much to expect in real 
situations. By solving Equations (2-1) and (2-2) for a three-point bend test, the maximum deflection at midspan, $w$, can be expressed as shown in Equation (2-4)

$$
w=\frac{P l}{4}\left(\frac{l^{2}}{12 E_{b} I}+\frac{1}{A G_{b}}\right)
$$

where $P$ is the applied load at the midpoint and $l$ is the effective length. Here, the term to the right side within parentheses represents the shear deformation effect coupled with the bending effect. By rearranging the terms in Equation (2-4), an equation for a straight line obtained as shown in Equation (2-5). In this equation, the slope is directly related to the flexural modulus, and the intercept is directly related to the section shear modulus (Bank 1989).

$$
\frac{4 A w}{P l}=\frac{1}{12 E_{b}}\left(\frac{l}{r}\right)^{2}+\frac{1}{G_{b}}
$$

A linear regression model can be used to produce a straight line fit through experimental data points on a plot of $(\mathrm{l} / \mathrm{r})^{2}$ versus $4 \mathrm{Aw} / \mathrm{Pl}$. From this plot, the section flexural and shear moduli can be calculated as shown in Equations (2-6) and (2-7), respectively (Bank 1989).

$$
\begin{aligned}
E_{b} & =\frac{1}{12 \times \text { slope }} \\
G_{b} & =\frac{1}{\text { intercept }}
\end{aligned}
$$

By accounting for the shear deformation, the section shear and flexural moduli can be determined with reasonable accuracy for a wide range of spans. The use of a full-section modulus is advantageous for designers because it eliminates the need for calculating the shear 
coefficient of the section, and also results in superior accuracy in calculating beam deflections using the Timoshenko Beam Theory (Bank 1989).

\subsection{Bending Behavior of GFRP Box Beams}

A limited amount of theoretical models have been developed for predicting the failure mode and critical flexural capacity of pultruded FRP box members. However, one of the early prediction models provided specifically for these types of specimens was developed by A. F. Johnson in 1985, which hypothesized that the failure of pultruded box members comprised of five distinct modes: (1) compression face buckling, (2) tension material failure, (3) compression material failure, (4) shear material failure, and (5) shear buckling in the side wall. This model assumes the interaction between flexural and shear failure modes to be nonexistent, thus the lowest calculated capacity between these failure modes is to be taken as the critical load. However, subsequent research has shown that other failure modes do exist in these complex materials with non-conventional shapes. Johnson's model, which was developed on the basis of linear elastic thin-wall theory, is applied as follows.

The ultimate capacity of the beam will be controlled by either a bending or shear failure, as determined by applying Equations (2-8) and (2-9) (Mottram 1991).

$$
\begin{gathered}
P_{B}=\left(\frac{16 a^{2} h}{3 l}\right) \varphi \sigma_{\max } \\
P_{S}=\frac{8}{3} a h \psi \tau_{\max }
\end{gathered}
$$

In which

$P_{B}=$ ultimate load at failure in bending

$P_{S}=$ ultimate load at failure in shear 
$a=$ side length

$h=$ wall thickness

$l=$ test span length

$\varphi=$ thin-walled compressive buckling coefficient, as calculated in Equation (2-10)

$\sigma_{\max }=$ material failure stress in either tension or compression

$\psi=$ thin-walled shear buckling coefficient

$\tau_{\max }=$ shear failure stress of the side wall material

For applying Equation (2-8), the thin-walled compressive buckling coefficient, $\varphi$, is calculated using Equation (2-10) (Mottram 1991).

$$
\varphi=\frac{1}{3} \pi^{2} K_{1}\left(\frac{h}{a}\right)^{2}\left[\frac{E_{L}}{\left(1-v_{L T} v_{T L}\right) \sigma_{\max }}\right]
$$

Where

$$
K_{1}=\frac{1}{2}\left[\left(\frac{D_{T}}{D_{L}}\right)^{0.5}+\frac{D_{O}}{D_{L}}\right]
$$

Where

$$
\begin{gathered}
D_{L}=\frac{E_{L} h^{3}}{\left(1-v_{L T} v_{T L}\right)} \\
D_{T}=\frac{E_{T} h^{3}}{\left(1-v_{L T} v_{T L}\right)} \\
D_{O}=\frac{v_{T L} E_{L} h^{3}}{\left(1-v_{L T} v_{T L}\right)}+\frac{G_{L T} h^{3}}{12}
\end{gathered}
$$

In which

$h=$ wall thickness

$a=$ side length 
$E_{L}=$ characteristic value of the longitudinal modulus

$v_{L T}=$ characteristic value of Poisson's ratio in the longitudinal direction

$v_{T L}=$ characteristic value of Poisson's ratio in the transverse direction

$G_{L T}=$ characteristic value of the in-plane shear modulus

$D_{L}, D_{T}, D_{O}=$ orthotropic plate flexural rigidities as calculated in Equations (2-12) through (2-14)

When $\varphi<1$, the mode of failure is characterized by compression buckling of the top face, and the ultimate bending capacity is calculated using Equation (2-8). When $\varphi>1$, the bending failure is controlled by the strength of the material, and the ultimate load is calculated using Equation (2-8) while taking $\varphi$ as equal to 1 (Mottram 1991).

For applying Equation (2-9), the thin-walled shear buckling coefficient, $\psi$, is calculated using Equation (2-15) (Mottram 1991).

$$
\psi=6.6 K_{2}\left(\frac{h}{a}\right)^{2}\left[\frac{E_{L}}{\left(1-v_{L T} v_{T L}\right) \sigma_{\max }}\right]
$$

Where

$$
K_{2}=\left(\frac{D_{T}}{D_{L}}\right)^{0.5}\left(\frac{D_{O}}{D_{L}}\right)^{0.5} f(\delta)
$$

Where

$$
\delta=\frac{\left(D_{L} D_{T}\right)^{0.5}}{D_{O}}
$$

and

$$
f(\delta)=\left\{\begin{array}{c}
\delta^{0.5}\left(0.62+\frac{0.38}{\delta}\right), \text { when } \delta>1 \\
0.89+0.04 \delta+0.07 \delta^{2}, \text { when } \delta<1
\end{array}\right.
$$

In which 
$h=$ wall thickness

$a=$ side length

$E_{L}=$ characteristic value of the longitudinal modulus

$v_{L T}=$ characteristic value of major Poisson's ratio

$v_{T L}=$ characteristic value of minor Poisson's ratio

$\tau_{\max }=$ shear failure stress of the side wall material

$D_{L}, D_{T}, D_{O}=$ orthotropic plate flexural rigidities as calculated in Equations (2-12) through (2-14)

When $\psi<1$, the mode of failure is characterized by shear buckling of the side walls and the ultimate shear capacity is calculated using Equation (2-9). When $\psi>1$, the shear failure is controlled by the strength of the material, and the ultimate load is calculated using Equation (2-9) while taking $\psi$ as equal to 1 (Mottram 1991).

Johnson's simplified design procedure was evaluated based on experimental testing by J. T. Mottram in 1991. Mottram collected experimental data from a series of twenty-three tests on four sizes of square pultruded box beams with side lengths ranging from $37.85 \mathrm{~mm}$ (1.5 in.) to $50.86 \mathrm{~mm}(2.0 \mathrm{in}$.$) and wall thicknesses ranging from 1.94 \mathrm{~mm}(0.076$ in.) to $6.14 \mathrm{~mm}(0.242$ in.). These specimens were constructed using vinylester resin and two different types of E-glass rovings. For additional details regarding the fiber architecture of the test specimens, please refer to the original document by Mottram (1991).

The test specimens were subjected to three-point bending at varying span-to-depth ratios. The experimental setup included flat end supports and a flat loading ram, each having a bearing surface length of $18 \mathrm{~mm}$ (0.71 in.) in order to prevent localized failure at these locations. Mottram cites previous issues of local material failure associated with cylindrical roller-type supports, and attests that the bearing surface length is an influential parameter on the critical 
capacity of pultruded members. The specimens were loaded at a constant displacement rate which varied from $0.05 \mathrm{~mm} / \mathrm{s}(0.002 \mathrm{in.} / \mathrm{s})$ or $0.1 \mathrm{~mm} / \mathrm{s}(0.004 \mathrm{in.} / \mathrm{s})$ at spans less than $900 \mathrm{~mm}$ (35.4 in.), to $0.2 \mathrm{~mm} / \mathrm{s}(0.008 \mathrm{in.} / \mathrm{s}$ ) for longer spans (Mottram 1991). Two of the box beam specimens included in the study do not meet the thin-wall criteria, therefore a thick-walled correction factor developed by Roark (Young 1989), as calculated in Equation (2-19), must be applied by multiplying the shear correction factor, $K_{S}$, by the thick-walled correction factor (Mottram 1991).

$$
1-2\left(\frac{h}{a}\right)+2\left(\frac{h}{a}\right)^{2}
$$

In order to apply the design equations of Johnson's model, the values of the orthotropic elastic constants $E_{L}, G_{L T}$, and $v_{L T}$ are required. For his analysis, Mottram considered the typical minimum values provided by the manufacturer based on coupon testing performed in accordance with ASTM test standards. Additionally, Mottram calculated properties from full-scale specimens using Bank's work (1989). The resulting full-section elastic properties obtained by Mottram, as well as the minimum design elastic properties provided by the manufacturer are given in the original paper by Mottram (1991). It should be noted that the full-section elastic modulus values obtained experimentally ranging from $22 \mathrm{kN} / \mathrm{mm}^{2}$ (3.19 Msi) to $35 \mathrm{kN} / \mathrm{mm}^{2}$ (5.08 Msi) was greater than that obtained through coupon testing by the manufacturer for all cases. Similarly, the shear modulus from full-scale testing ranging from $2.5 \mathrm{kN} / \mathrm{mm}^{2}(0.36 \mathrm{Msi})$ to $6.3 \mathrm{kN} / \mathrm{mm}^{2}(0.91 \mathrm{Msi})$ was greater than the manufacturers design value for nearly all samples, with the exception of the $37.85 \mathrm{~mm} \times 37.85 \mathrm{~mm} \times 1.94 \mathrm{~mm}(1.5 \mathrm{in} . \times 1.5 \mathrm{in} . \times 0.076$ in.) specimen which resulted in a slightly lower value. 
During his evaluation, Mottram (1991) discovered that by equating Equations (2-8) and (2-9) and solving for the critical length, it could be shown that the span-to-depth ratio must be less than 7 in order for a shear failure to occur based on this model. Subsequent research suggests the threshold value of the span-to-depth ratio for shear failure to occur is more likely in the range of 10 to 12. An analysis of his experimental observations also resulted in a number of conclusions. First, Mottram noted that when the span-to-depth ratio fell within a range of 17.7 to 27.8, the failure mode was instantaneous and occurred in the compression flange beneath the point of load application, with longitudinal cracks at the reentrant corners propagating towards the outer supports, and transverse cracks visible in the top flange. One specimen, measuring $50.30 \mathrm{~mm} \times 50.30 \mathrm{~mm} \times 6.08 \mathrm{~mm}(2.0$ in. $\times 2.0$ in. $\times 0.24$ in. $)$ exhibited failure in the tension flange at the highest experimental span-to-depth ratio of approximately 28. At these higher spans, the ultimate failure of the two thick-walled specimens appeared to be controlled by the strength in compression, whereas the failure mode of the two thin-walled samples appeared to be controlled by buckling of the compression face (Mottram 1991). Furthermore, Mottram observed that for shorter spans, where the span-to-depth ratio ranged from 5.9 to 11.9 , the failure mode was one which was not included in Johnson's design model. In the two thin-walled specimens, the failure mode was characterized by longitudinal cracks at the junction of the compression flange and the side walls. These cracks allowed for the side walls to detach from the top flange and move outward, thus preventing a catastrophic failure (Mottram 1991). For the thick-walled specimens tested at shorter spans, the failure mode was a localized punching failure of the loading nose through the top face (Mottram 1991). Although no specific criteria was specified, those specimens characterized by Mottram (1991) as being thick-walled each possessed a depth- 
to-thickness ratio of less than 10 . These specimens resulted in undesirable failure modes which were not included in the initial development of the simplified Johnson design model.

Table 2-1 Comparison for simplified design analysis for ultimate load (modified from Mottram 1991)

\begin{tabular}{|c|c|c|c|c|c|c|c|c|c|c|}
\hline Specimen & $\begin{array}{l}\text { Span } \\
(\mathbf{m m})\end{array}$ & $P_{B} \exp (\mathrm{kN})$ & $\begin{array}{c}\sigma_{c, \max } \\
\left(\mathbf{N} / \mathbf{m m}^{2}\right)\end{array}$ & $\begin{array}{c}P_{B}(\mathbf{a}) \\
(\mathrm{kN})\end{array}$ & $\begin{array}{c}P_{B}(\mathbf{b}) \\
(\mathbf{k N})\end{array}$ & $\begin{array}{c}P_{B}(\mathbf{c}) \\
(\mathbf{k N})\end{array}$ & $\begin{array}{c}P_{B}(\mathrm{~d}) \\
(\mathrm{kN})\end{array}$ & $\psi$ & $\begin{array}{c}P_{S}(\mathrm{e}) \\
(\mathrm{kN})\end{array}$ & $\begin{array}{l}I_{\text {crit }}(\mathbf{f}) \\
(\mathrm{mm})\end{array}$ \\
\hline B38/34 & 900 & $1.78,1.90,1.60$ & 239,219 & 1.44 & 1.23 & 1.47 & 1.26 & 2.29 & 11.75 & 94 \\
\hline B38/34 & 400 & 2.72 & 237,242 & 3.24 & 2.78 & 3.32 & 2.84 & $(1)$ & & \\
\hline B38/34 & 300 & $2.38,2.40$ & $(240)(\mathrm{g})$ & 4.32 & 3.70 & 4.41 & 3.78 & & & \\
\hline B44/32 & 900 & $19.4,18.6,18.2$ & 431,417 & 27.8 & 18.2 & 21.0 & 13.8 & 21.8 & 43.3 & 327 \\
\hline B44/32 & 300 & $26.6,27.6$ & $\begin{array}{c}417,305 \\
(390)\end{array}$ & 83.5 & 54.7 & 63.1 & 41.3 & $(1)$ & & \\
\hline B51/45 & 900 & $7.50,6.75,7.52$ & 365,327 & 7.15 & 5.94 & 4.97 & 4.13 & 3.65 & 25.2 & 213 \\
\hline B51/45 & 500 & 7.56 & (340) & 12.9 & 10.7 & 8.92 & 7.41 & $(1)$ & & \\
\hline B51/45 & 300 & $11.8,9.74$ & & 21.5 & 17.8 & 14.8 & 12.3 & & & \\
\hline B50/38 & 1400 & 9.2 & 144,251 & 10.4 & 7.22 & 11.4 & 7.91 & 12.9 & 48.9 & 206 \\
\hline $\mathrm{B} 50 / 38$ & 1000 & 11.75 & 174,142 & 14.6 & 10.1 & 16.1 & 11.1 & $(1)$ & & \\
\hline $\mathrm{B} 50 / 38$ & 600 & $13.6,13.5$ & (180) & 24.3 & 16.9 & 26.7 & 18.5 & & & \\
\hline B50/38 & 400 & $15.8,14.8$ & & 36.5 & 25.6 & 40.4 & 28.0 & & & \\
\hline
\end{tabular}
(a) measured data in Equations (2-8) and (2-10)
(b) measured data applying thick-walled correction factor to Equation (2-8)
(c) nominal design data in Equations (2-8) and (2-10)
(d) nominal design data applying thick-walled correction factor to Equation (2-8)
(e) measured data (except $\tau_{\max }$ ) in Equation (2-9)
(f) from measured data
(g) mean compressive strength of specimens is given in parentheses

An evaluation of the Johnson (1985) prediction model versus the experimental data revealed largely inconsistent and often inaccurate results, as shown in Table 2-1. As one would expect with the model being developed based on linear elastic thin-walled theory, the prediction results for the thin-walled samples were significantly more accurate than the thicker-walled profiles. It must also be noted that results of the prediction model were dramatically improved when utilizing the experimentally obtained elastic strength properties from full-section testing. Using this data, prediction was largely within \pm 20 percent of the experimental capacity at spanto-depth ratios greater than 10 for thin-walled samples. The results at long spans were drastically 
improved for the thicker box specimens with the application of the thick-walled correction factor shown in Equation (2-19), particularly the $44.12 \mathrm{~mm} \times 44.12 \mathrm{~mm} \times 6.14 \mathrm{~mm}(1.74 \mathrm{in} . \times 1.74 \mathrm{in}$. $\times 0.24$ in.) specimen which exhibited a maximum error of -6.2 percent at a span-to-depth ratio of 20.45, with one experimental replication matching the predicted value. Overall, the results based on the manufacturers' design values for the elastic properties yielded highly inaccurate results. The prediction model also proved inapplicable for short span-to-depth ratios (less than 11.9), where the predicted values were always much higher than the experimental values (Mottram 1991). Lastly, none of the twenty-three test specimens underwent a shear type failure mode, and the predicted value using Equation (2-9) was always at least double that of the experimental value, even at span-to-depth ratios as low as 5.88 .

Another prediction model worth discussing was developed by László Kollár (2003), which aimed to calculate the local buckling capacity of a number of FRP cross-sections, including box specimens. Kollár notes that this type of failure mode is most likely to occur at short span-to-depth ratios, and could occur at an intermediate span, where the combined interaction of local and global buckling modes must be considered (Barbero 2000). The model was developed based on the plate buckling theory originally developed by Bleich (1952). Due to the complexity of the model, Kollár notes that no explicit expressions which are simplistic enough to be used in a practical design scenario could be developed.

To begin, each wall of the specimen is assumed to be an individual plate with simplysupported edges. By utilizing Bleich's classical solving method, the critical stress can be calculated for each segment. By determining the critical axial strain in the webs and flanges, the component which is most susceptible to local buckling can be identified (Kollár 2003). This critical component is then evaluated by treating the wall segment as a plate which is rotationally 
restrained at edges that meet with adjacent wall segments. This restraint is dependent on the wall configuration, and two different cases are considered by Kollár (2003). The first case considers both edges to be restrained by neighboring wall segments, and the second case assumes one edge to be free; for a box member, the first case applies. In this scenario, the deformed shape of the segment is assumed to be cylindrical (Bleich 1952). Thus, the applied moment along the edge, $M_{y}$, can be expressed as shown in Equation (2-21)

$$
M_{y}=(\mp) k \frac{\partial w}{\partial y}
$$

Where

$$
k_{o}=\frac{c\left(D_{22}\right)_{r s}}{L_{r s}}
$$

In which the subscript $r$ s refers to the restraining wall, $w$ refers to the web, and

$k_{o}=$ rotational spring constant

$c=$ constant dependent on the edge conditions of the restraining wall

$D_{22}=$ element of the laminated plate bending stiffness matrix, calculated using Equation (2-22)

$L_{r s}=$ length of the restraining segment.

The second element of the laminated plate bending stiffness matrix for an orthotropic box members is calculated as shown in the following equation

$$
D_{22}=\frac{E_{2} h^{3}}{12\left(1-v^{2}\right)}
$$

In which

$E_{2}=$ transverse Young's modulus

$h=$ thickness of the plate

$v=$ Poisson's ratio 
Under these conditions, both webs will buckle simultaneously, leading to equal bending moments at each edge of the restraining segment. This expression is valid only in the absence of an applied axial load (Kollár 2003). Thus, the effect of axial loading is accounted for through the use of an amplification factor, $r$, developed by Timoshenko and Gere (1961), shown in Equation $(2-23)$.

$$
r=\frac{1}{1-\frac{\left(N_{x}\right)_{r s}}{\left(N_{x c r}\right)_{r S}^{S S}}}
$$

In which

$\left(N_{x}\right)_{r s}=$ applied buckling stress on the wall segment

$\left(N_{x c r}\right)_{r S}^{S S}=$ buckling stress of the simply supported restraining wall segment

It should be noted that when the applied axial load is zero, $r=1$, and when the applied axial load and buckling load are equal, $r=\infty$. In applying the amplification factor, the rotational spring constant is now expressed as shown in Equation (2-24) (Kollár 2003).

$$
k=\frac{c\left(D_{22}\right)_{r s}}{L_{r s}} \frac{1}{r}
$$

In this scenario, the resulting axial strains of the restraining segment and the buckled wall segments are the same, thus the expression for $\left(N_{x}\right)_{r s}$ can be written as shown in Equation (2-25) below (Kollár 2003).

$$
\left(N_{x}\right)_{r s}=\left(N_{x c r}\right)_{b u} \frac{\left(a_{11}\right)_{b u}}{\left(a_{11}\right)_{r s}}
$$

Where

$\left(N_{x c r}\right)_{b u}=$ buckling stress of the rotationally restrained buckled wall segment $\left(a_{11}\right)_{b u}=$ tensile compliance of the buckled plate 
$\left(a_{11}\right)_{r s}=$ tensile compliance of the restraining wall segment

The value of $\left(N_{x c r}\right)_{b u}$ is not known from theoretical deduction, and therefore must be approximated by the buckling stress of a simply supported wall, $\left(N_{x c r}\right)_{b u}^{s s}$. By making this approximation, the equation for the amplification factored is rewritten as shown in Equation (2-26) (Kollár 2003).

$$
r=\frac{1}{1-\frac{\left(N_{x c r}\right)_{b u}^{S S}\left(a_{11}\right)_{b u}}{\left(N_{x c r}\right)_{r s}^{S S}\left(a_{11}\right)_{r s}}}
$$

By applying this same procedure to a box specimen subjected to pure bending about the $y$-axis, the buckling stresses of the flange and web are calculated as shown in Equations (2-27) and (2-28), respectively (Kollár 2003).

$$
\begin{gathered}
\left(N_{x c r}\right)_{f}^{s s}=\frac{\pi^{2}}{b_{f}^{2}}\left(2 \sqrt{\left(D_{11}\right)_{f}\left(D_{22}\right)_{f}}+2\left[\left(D_{12}\right)_{f}+2\left(D_{66}\right)_{f}\right]\right) \\
\left(N_{x c r}\right)_{w}^{S S}=\frac{\pi^{2}}{b_{w}^{2}}\left(13.9 \sqrt{\left(D_{11}\right)_{w}\left(D_{22}\right)_{w}}+11.1\left[\left(D_{12}\right)_{w}+2\left(D_{66}\right)_{w}\right]\right)
\end{gathered}
$$

In which the subscripts $f$ and $w$ refer to the flange and web, and $b=$ base length $D_{i j}=$ elements of the laminated plate bending stiffness matrix

When $\left(N_{x c r}\right)_{f}^{S S}\left(a_{11}\right)_{f}<\left(N_{x c r}\right)_{w}^{s S}\left(a_{11}\right)_{w}$, the flange will be the first component to buckle. In this event, it is assumed that the tension flange stabilizes the web. Therefore, the constant $c$ used for calculating the rotational spring constant is equal to 4 , and the formula for calculating the local buckling stress of the box specimen is written as shown in Equation (2-29) (Kollár 2002). 


$$
N_{x c r}=\pi^{2}\left(2 \sqrt{1+4.139 \xi} \sqrt{D_{11} D_{22}}+\left(2+0.62 \xi^{2}\right)\left(D_{12}+2 D_{66}\right)\right) / L_{y}^{2}
$$

Where

$$
\xi=\frac{1}{(1+10 \zeta)}
$$

Where

$$
\zeta=\frac{D_{22}}{\left(k L_{y}\right)}
$$

In which

$L_{y}=$ plate width

$D_{i j}=$ elements of the laminated plate bending stiffness matrix

Conversely, when $\left(N_{x c r}\right)_{f}^{S S}\left(a_{11}\right)_{f}>\left(N_{x c r}\right)_{w}^{S S}\left(a_{11}\right)_{w}$, the web will be the first to buckle. Under this condition, the rotational spring constant, $k$, can be conservatively taken as zero, thus resulting in Equation (2-32) for calculating the local buckling stress of the box specimen (Lekhnitskii 1968).

$$
N_{x c r}=\pi^{2}\left(13.9 \sqrt{D_{11} D_{22}}+11.1\left(D_{12}+2 D_{66}\right)\right) / L_{y}^{2}
$$

In which

$L_{y}=$ plate width

$D_{i j}=$ elements of the laminated plate bending stiffness matrix

Kollár did a numerical analysis using the ANSYS finite element modelling software, and compared the results of this model to the prediction equations. The box member used for the FE model was taken from a previous experiment by Qiao et al. (2001). This particular specimen measured $203.2 \mathrm{~mm} \times 101.6 \mathrm{~mm} \times 6.35 \mathrm{~mm}$ ( 8 in. $\times 4$ in. $\times 0.25$ in.) and had orthotropic bending stiffness properties $D_{11}=444 \mathrm{~N} \cdot \mathrm{m}(327.5 \mathrm{lb} \cdot \mathrm{ft}), D_{22}=461 \mathrm{~N} \cdot \mathrm{m}(340.0 \mathrm{lb} \cdot \mathrm{ft}), D_{12}=103 \mathrm{~N} \cdot \mathrm{m}$ 
$(75.97 \mathrm{lb} \cdot \mathrm{ft})$, and $D_{66}=107 \mathrm{~N} \cdot \mathrm{m}(78.92 \mathrm{lb} \cdot \mathrm{ft})$. The prediction equation resulted in a critical buckling stress of $1,790 \mathrm{kN} / \mathrm{m}(122.7 \mathrm{kip} / \mathrm{ft})$, slightly less than the critical buckling stress calculated using the FE model of 1,960 kN/m (134.3 kip/ft) for a resulting error of -8.7 percent (Kollár 2003). One source of this error which is noted by Kollár is a failure to account for the resin-rich regions at the web-flange junctions, where the stiffness is lower than the overall stiffness of the plate. For improved accuracy, the reduced stiffness in this region must be accounted for through the use of a stress concentration factor.

Another prediction model which focuses on the accurate prediction of one particular failure mode through the use of DYNA3D finite element modelling software was developed by Palmer, Bank, and Gentry (1998). The authors aimed to recreate through a series of experimental tests what they described as a "progressive tearing failure mode," characterized by a separation of the web and flange at the junctions, typically beginning at the point of load application and propagating outwards towards the end supports (Palmer et al. 1998). Testing was performed on a $76.2 \mathrm{~mm} \times 76.2 \mathrm{~mm} \times 6.25 \mathrm{~mm}(3 \mathrm{in} . \times 3$ in. $\times 0.25 \mathrm{in}$. $)$ pultruded box section constructed of glass fiber rovings and a vinylester resin at a span length of $1905 \mathrm{~mm}$ (75 in.), for a resulting span-to-depth ratio of 25 . The end supports were two standard W6x9 steel I-sections with a mechanism which elastically restrained the specimen from rotation at the supports. The load was applied at midspan with a hydraulic loading nose $76.2 \mathrm{~mm}$ (3 in.) in diameter. The load was applied at a constant rate of displacement ranging from $5 \mathrm{~mm} / \mathrm{min}(0.20 \mathrm{in} . / \mathrm{min})$ to $10 \mathrm{~mm} / \mathrm{min}$ (0.39 in./min) (Palmer et al. 1998).

During testing, the load and vertical deflection were continuously recorded. As a result, Palmer et al. (1998) noted a stiffening-softening-stiffening trend in load versus deflection plot for all specimens, although each case exhibited mostly elastic behavior up until the first point of 
material rupture. Figure 2-1 demonstrates the typical rupture mode which marked the end of the stiffening phase during experimental evaluation (Palmer et al. 1998).

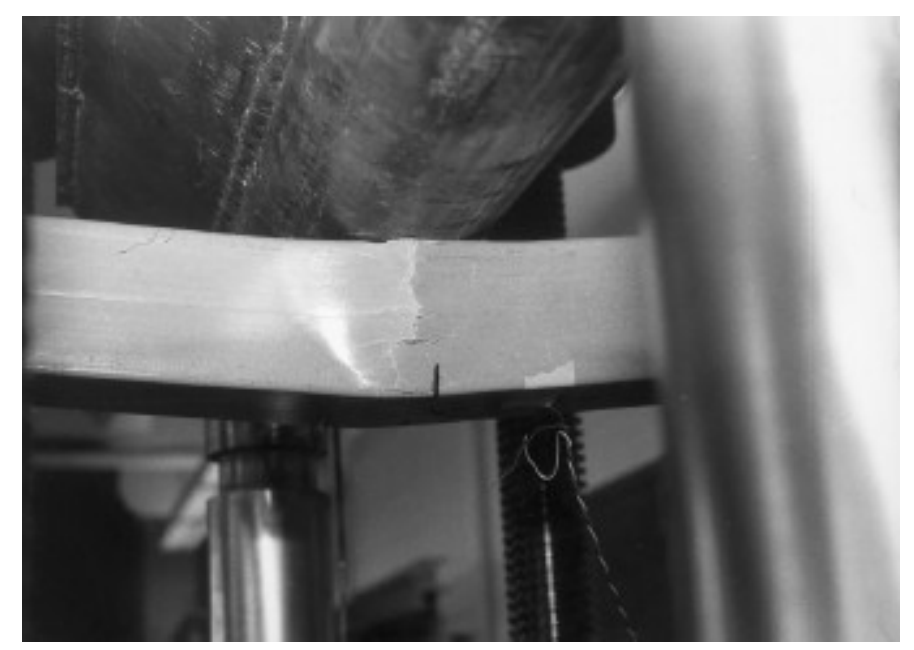

Figure 2-1 Beam at end of first stiffening phase (Palmer et al. 1998)

At this point of rupture, a sudden reduction in the bending stiffness is noted. As can be seen in the photo, there is an initial delamination of the webs from the compression flange directly under the applied load, with visible fiber breakage. As the load is further applied, lateral deflection of the webs occurs leading to continued tearing of the wall and an eventual delamination of the tension flange, as shown in Figure 2-2. As the loading ram continues to displace vertically, the tearing at the lower web-flange junctions propagates outward. This is a failure mode which is unique to pultruded thin-walled FRP box members under certain conditions (Palmer et al. 1998). 


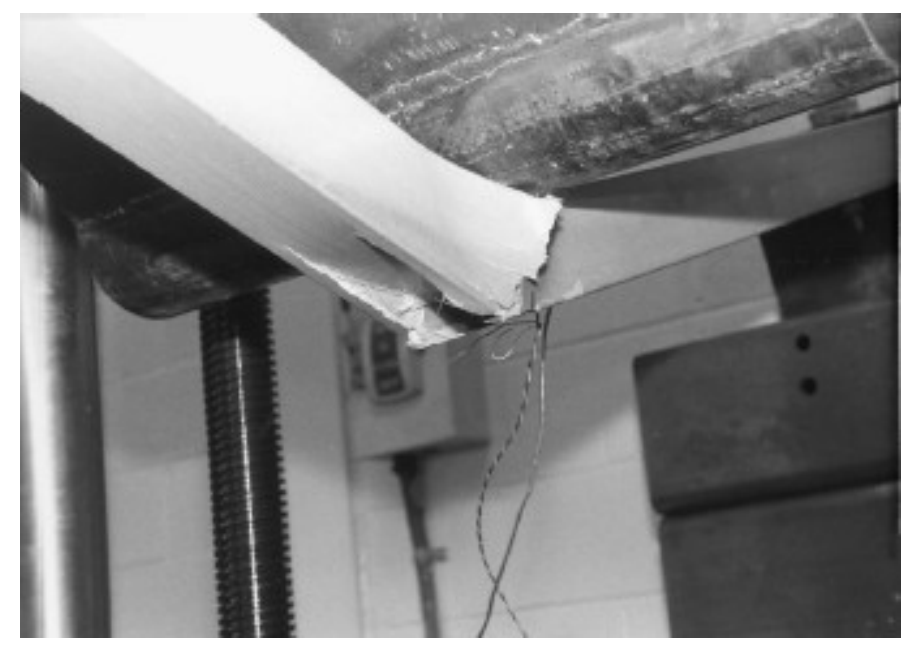

Figure 2-2 Tearing damage visible in the tension flange (Palmer et al. 1998)

As the tearing further progressed, a slight stiffening effect could be observed due to transfer of the load to the flanges, thus helping to prevent complete catastrophic failure. Palmer et al. (1998) also observed that occurrence of this progressive tearing mode is also a function of the wall thickness, where very little tearing occurred in thicker-walled specimens. Thus, the authors hypothesized that beyond a certain ratio of an unknown cross sectional dimension-towall thickness, the tearing mode of failure would be nonexistent and give way to a more catastrophic failure mode (Palmer et al. 1998).

Palmer et al. (1998) also developed a finite element model using LS-DYNA3D to simulate the same test as performed in experimental work. The elastic material stiffness properties used in constructing the model were supplied by the manufacturer. A summary of these material properties and their equivalent values in U.S. customary units can be seen in Table 2-2. In this table $Y$ is the transverse strength of the material, $T$ is the shear strength of the material, and $\rho$ is the density (Palmer et al. 1998). 
Table 2-2 Elastic constants of composite material used in simulations (Palmer et al. 1998)

\begin{tabular}{ccc}
\hline $\boldsymbol{E}_{\boldsymbol{x} \boldsymbol{x}}$ & $20.69 \mathrm{GPa}$ & $3.00 \mathrm{Msi}$ \\
$\boldsymbol{E}_{\boldsymbol{y} \boldsymbol{y}}$ & $6.89 \mathrm{GPa}$ & $1.00 \mathrm{Msi}$ \\
$\boldsymbol{G}_{\boldsymbol{x} \boldsymbol{y}}$ & $2.89 \mathrm{GPa}$ & $0.42 \mathrm{Msi}$ \\
$\boldsymbol{y}_{\boldsymbol{y} \boldsymbol{x}}$ & 0.12 & 0.12 \\
$\boldsymbol{Y}$ & $0.055 \mathrm{GPa}$ & $7.97 \mathrm{ksi}$ \\
$\boldsymbol{T}$ & $0.041 \mathrm{GPa}$ & $5.95 \mathrm{ksi}$ \\
$\boldsymbol{\rho}$ & $1.94 \times 10^{3} \mathrm{~kg} / \mathrm{m}^{3}$ & $121 \mathrm{lb} / \mathrm{ft}^{3}$ \\
\hline
\end{tabular}

For further details regarding the development of the FE model used in this study, please refer to the original paper by Palmer et al. (1998). The model was run four times at varying standard displacement rates of 5,50,100, and $500 \mathrm{~cm} / \mathrm{s}(1.97,19.7,39.4$, and $197 \mathrm{in} / \mathrm{s})$. The results of the $\mathrm{FE}$ model at 50 and $100 \mathrm{~cm} / \mathrm{s}$ matched quite well with the experimental data. Although the numerical results produced good correlation, the tearing pattern observed during experimental evaluation was not consistently matched for the four different loading ram displacement rates used in the FE simulation. For the three slowest displacement rates, the tearing mode occurred only at the junctions of the webs and compression flange. Surprisingly, the fastest displacement rates produced the worst correlation numerically, but resulted in a tearing mode of failure which most resembled the experimental observations. A conclusion can be drawn that the beam response depends largely on the displacement rate of the applied load (Palmer et al. 1998).

A fourth and final model related to pultruded FRP box beams discussed in this literature review is based on a buckling analysis using finite element modelling software performed by Chen and El-Hacha (2012). In this study, and experimental evaluation was performed using a $152.4 \mathrm{~mm} \times 224.8 \mathrm{~mm} \times 11.11 \mathrm{~mm}(6.0 \mathrm{in} \times 8.9 \mathrm{in} . \times 0.44$ in. $)$ pultruded GFRP box specimen subjected to four-point bending. The sample was tested at a span length of $2900 \mathrm{~mm}$ (114.2 in.), 
with the two point loads applied $300 \mathrm{~mm}$ (11.8 in.) from each outer support, for a resulting spanto-depth ratio of 12.9. The ends of the specimen were filled with Ultra-High Performance Concrete (UHPC) to prevent localized crushing at the end supports. No discussion of the stiffening effect caused by the UHPC end blocks is provided by the authors. The resulting failure mode of this testing varied, including: compressive failure in the top flange, buckling of the webs at midspan, and rupture of the web-flange junctions (Chen and El-Hacha 2012).

For comparison, a finite element model was constructed using the ABAQUS 6.9 software. The elastic constants for developing the model were obtained using coupon-level tension testing. The results of this testing revealed that the longitudinal modulus of elasticity differed for the flanges, the webs, and the corners; thus, these components of GFRP box beam were each modelled separately. An initial preload of $100 \mathrm{kN}$ (22.5 kipf) was applied to the model prior to the buckling analysis in order to incorporate geometric nonlinearities caused by large deformations into the model to increase the numerical accuracy of the prediction. The failure mode exhibited by the FE model was compared with experimental observations. Many of the features of the deformed shape agree with the experimental, including: transverse displacement of the beam specimen away from the longitudinal axis of symmetry, significant vertical deformation of the compression flange near the points of load application, and buckling of the web below the point loads (Chen and El-Hacha 2012).

After confirming the accuracy of the failure mode, predicted critical buckling load, $P_{c r}$, was calculated to be $138.8 \mathrm{kN}$ (31.2 kipf) based on the FE model. This resulted in an overprediction of the experimental data by 12 percent. The authors believe this overestimation is the result of a failure to account for the additional geometric nonlinearity due to the coupling of inplane membrane deformation with the global deformation of the specimen; a further explanation 
for this error is manufacturing imperfections (Chen and El-Hacha 2012). The use of a preload step helps to account for the imperfect linear-elastic behavior which is characteristic of pultruded FRP members. Overall, the use of the classic linear eigenvalue approach with the incorporation of the preload step shows good approximation for the critical buckling load of the pultruded GFRP box member used in this study.

\subsection{LTB of GFRP Open Sections}

A significant amount of research has been carried out relating to lateral-torsional buckling (LTB) of FRP composite members. The behavior of pultruded GFRP open sections, such as channels and wide flange beams, is often influenced by large local deformations due to a high strength-to-stiffness ratio, making them highly susceptible to global buckling failures such as LTB (Mottram 1991). While studies on LTB of steel open sections has been carried out for the better part of the last century, some of the early work with LTB of polymeric composites was performed by J. T. Mottram in the early part of the 1990's. Mottram (1992) recognized the importance in understanding the LTB behavior of pultruded FRP members from a design standpoint. Mottram began his research by modifying the classic one-dimensional formula for calculating the critical LTB moment for a doubly-symmetric isotropic steel I-beam originally developed by Timoshenko and Gere (1961), shown in Equation (2-33), to be used in the design of an orthotropic member such as pultruded FRP beams. This equation has been proven applicable for use in the design of slender isotropic beams with concentrated loading applied at the centroid, perpendicular to the strong axis (Mottram 1992).

$$
M_{c r}=\frac{C_{1} \pi}{k l}\left[E I_{y y} G J\right]^{0.5}\left[1+\frac{\pi^{2} E I_{w}}{\left(k_{w} l\right)^{2} G J}\right]^{0.5}
$$


In which

$E I_{y y}=$ minor axis flexural rigidity

$G J=$ torsional rigidity

$E I_{w}=$ warping rigidity, where $I_{w}=$ warping constant $=D^{2} I_{f} / 4$

$C_{1}=$ factor to allow for the type of loading

$k l=$ effective length, where $k$ refers to the end support restraint about the minor $y-y$ axis, and varies from 1.0 for no fixity to 0.5 for full fixity

$k_{w}=$ constant related to end support warping, which varies from 1.0 for no fixity to 0.5 for full fixity

$D=$ depth of beam

$I_{f}=$ second moment of area about the minor $y-y$ axis

The modifications made to Equation (2-33) by Mottram (1992) were based on the following assumptions. Figure 2-3 demonstrates the alignment of the $x y z$ plane for this discussion.

- The end supports restrain the beam specimen from twisting about the $z-z$ axis.

- The end supports do not allow for rotation about the minor $y-y$ axis.

- The end supports allow for free rotation about the major $x-x$ axis.

- The beam is prevented from warping at the end supports. 


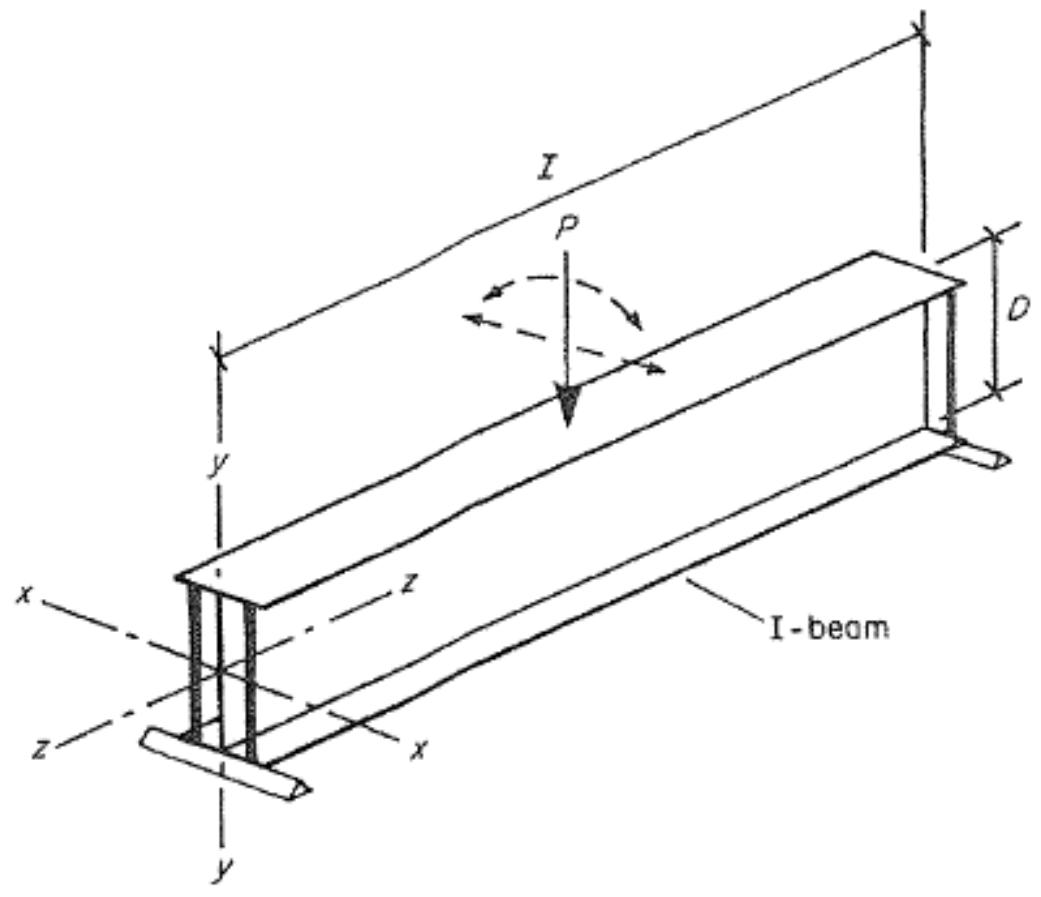

Figure 2-3 Schematic representation of test setup (Mottram 1992)

By making these assumptions and substituting in the relevant elastic strength properties for an orthotropic member, the resulting formula for the critical LTB capacity of a pultruded FRP Isection can be written as shown in Equation (2-34) (Mottram 1992). In this formulation, the values of $k$ and $k_{w}$ have both been set equal to 0.5 , as is practiced in the MMFG design manual (1988).

$$
P_{c r}=\frac{8.56 \pi}{l^{2}}\left[E_{z, y y} I_{y y} G_{x y} J\right]^{0.5}\left[1+\frac{4 \pi^{2} E_{z, y y} I_{w}}{l^{2} G_{x y} J}\right]^{0.5}
$$

In which

$E_{z, y y} I_{y y}=$ minor axis flexural rigidity

$G_{x y} J=$ torsional rigidity

$E_{z, y y} I_{w}=$ warping rigidity, where $I_{w}=$ warping constant $=D^{2} I_{f} / 4$

$C_{1}=1.07$ for central point load 
$l=$ span length

$D=$ depth of beam

$I_{f}=$ second moment of area about the minor $y-y$ axis

In addition to the previously mentioned assumptions, the development of the critical buckling load formulation shown in Equation (2-34) is also inherently dependent on the following fundamental assumptions associated with the classic beam flexure formula.

- The beam is initially straight.

- The beam behaves linear elastically.

- The loading initially acts in the plane of the web.

- The beam is initially free of residual stresses.

Like Equation (2-33), the modified equation is only valid for transverse loading applied at the centroid of the beam specimen. It should also be noted here that the subscripts $x x$ and $y y$ denote properties which act either in the major or minor axis direction; likewise, the subscript $x y$ refers to the in-plane shear modulus associated with torsion about the $z$-axis (Mottram 1992).

In order to apply Equation (2-34), the values of the orthotropic elastic strength properties $E_{z, y y}$ and $G_{x y}$ must be known. For his analysis, Mottram considered both the design values provided by the manufacturer based on coupon testing performed in accordance with ASTM test standards for measuring the elastic moduli, and a non-standard test method of full-scale members for determining the shear modulus. Full-scale section elastic properties were also calculated by Mottram (1992) through the use of the procedure developed by Bank (1989), which was discussed in detail in Section 2.2. As one would expect, this analysis resulted in an elastic modulus value higher than that of the more conservative design values provided by the 
manufacturer. However, the shear modulus obtained by Mottram (1992) through his own analysis was actually lower than the manufacturer's design value. This difference is attributed to the use of a different method by the manufacturer for calculating the contribution of shear deformation, as described in detail in the paper by Mottram (1992). At the time of his study, no standard method for calculating the shear modulus existed. Based on the results of his own analysis of the elastic section properties, Mottram notes that the ratio of the shear-to-longitudinal elastic modulus is less than 0.07, which exemplifies the importance of including shear deformation in the analysis of pultruded FRP composite members (Mottram 1992). It should be pointed out that more recent studies have found the value of this ratio to lie somewhere in the range of 0.10 to 0.14 .

The results of the prediction equation are compared against experimental results from three-point bend tests performed on three wide flange test specimens measuring $102 \mathrm{~mm} \times 52$ $\mathrm{mm} \times 6.4 \mathrm{~mm}(4$ in. $\times 2$ in. $\times 0.25$ in. $)$. Testing was carried out at a span length of $1500 \mathrm{~mm}(63$ in.). The transverse load was transferred to the top flange using a $51 \mathrm{~mm} \times 57 \mathrm{~mm}(2 \mathrm{in} . \times 2.24$ in.) metal plate, which served to reduce the bearing stress in order to prevent the possibility of a localized failure. A $16 \mathrm{~mm}$ (0.63 in.) ball bearing rested in a rounded cup at the center of the load transfer plate, thus allowing for a moving point of contact. The initial point of load application was at the centroid. As assumed in the development of the prediction equation, the end supports prevented the test specimen from twisting. Lateral displacement was measured using a $25 \mathrm{~mm}$ strain gage displacement transducer. The loading ram was applied at a constant rate of displacement set to $0.02,0.05$, or $0.1 \mathrm{~mm} / \mathrm{sec}$. A total of 35 tests were performed, and the buckling load was defined as the point when lateral displacement reached $2 \mathrm{~mm}(0.08 \mathrm{in}$.). This was assumed for convenience, and also because the theoretical model assumes the lateral 
displacement to be infinitesimally small (Mottram 1992). A summary of the measured section properties and resulting experimental buckling load values can be seen in Table 2-3.

Table 2-3 Section properties and critical load values for I102/51/6 beams at $1500 \mathrm{~mm}$ span (modified from Mottram 1992)

\begin{tabular}{|c|c|c|c|c|c|}
\hline Specimen & $\mathrm{E}_{\mathrm{z}}(\mathrm{GPa})$ & $\begin{array}{c}\mathbf{G}_{\mathrm{xz}} \\
(\mathrm{GPa})\end{array}$ & $\begin{array}{l}\text { Stroke Rate } \\
(\mathrm{mm} / \mathrm{s})\end{array}$ & $\begin{array}{l}\text { Maximum } \\
\text { Load }(k N)\end{array}$ & $\begin{array}{l}\text { Mode } \\
\text { Shape }\end{array}$ \\
\hline \multirow{9}{*}{$\mathbf{I 1 0 2} / 51 / 6 \mathrm{~A}$} & 22.5 & 1.3 & 0.05 & 3.85 & Third \\
\hline & 22.5 & 1.3 & 0.05 & 3.22 & Third \\
\hline & 22.5 & 1.3 & 0.05 & 3.45 & Third \\
\hline & 22.5 & 1.3 & 0.05 & 4.05 & Third \\
\hline & 22.5 & 1.3 & 0.02 & 3.95 & Third \\
\hline & 22.5 & 1.3 & 0.10 & 4.97 & Third \\
\hline & 22.5 & 1.3 & 0.05 & 5.74 & Third \\
\hline & 22.5 & 1.3 & 0.05 & 2.80 & First \\
\hline & 22.5 & 1.3 & 0.05 & 4.25 & Third \\
\hline \multirow{14}{*}{$\mathrm{I} 102 / 51 / 6 \mathrm{~B}$} & 22.6 & 1.2 & 0.10 & 3.75 & Third \\
\hline & 22.6 & 1.2 & 0.10 & 3.67 & Third \\
\hline & 22.6 & 1.2 & 0.05 & 2.82 & Third \\
\hline & 22.6 & 1.2 & 0.10 & 3.29 & Third \\
\hline & 22.6 & 1.2 & 0.05 & 2.81 & Third \\
\hline & 22.6 & 1.2 & 0.10 & 3.24 & Third \\
\hline & 22.6 & 1.2 & 0.05 & 5.75 & Third \\
\hline & 22.6 & 1.2 & 0.10 & 4.93 & Third \\
\hline & 22.6 & 1.2 & 0.05 & 2.99 & First \\
\hline & 22.6 & 1.2 & 0.02 & 2.92 & First \\
\hline & 22.6 & 1.2 & 0.05 & 2.94 & First \\
\hline & 22.6 & 1.2 & 0.05 & 3.60 & First \\
\hline & 22.6 & 1.2 & 0.05 & 2.84 & First \\
\hline & 22.6 & 1.2 & 0.05 & 2.91 & First \\
\hline \multirow{11}{*}{$\mathrm{I} 102 / 51 / 6 \mathrm{C}$} & 22.3 & 1.2 & 0.05 & 2.99 & First \\
\hline & 22.3 & 1.2 & 0.05 & 2.88 & First \\
\hline & 22.3 & 1.2 & 0.05 & 4.63 & Third \\
\hline & 22.3 & 1.2 & 0.05 & 3.76 & Third \\
\hline & 22.3 & 1.2 & 0.05 & 3.68 & Third \\
\hline & 22.3 & 1.2 & 0.05 & 3.42 & Third \\
\hline & 22.3 & 1.2 & 0.05 & 3.37 & First \\
\hline & 22.3 & 1.2 & 0.05 & 3.30 & First \\
\hline & 22.3 & 1.2 & 0.05 & 3.48 & First \\
\hline & 22.3 & 1.2 & 0.10 & 3.30 & First \\
\hline & 22.3 & 1.2 & 0.10 & 3.19 & First \\
\hline
\end{tabular}


As shown in the table, the recorded experimental buckling loads ranged from $2.80 \mathrm{kN}$ (629.5 lbf) to $5.75 \mathrm{kN}(1292.6 \mathrm{lbf})$, thus signifying the sensitivity of the buckling behavior based on potential small differences in the test setup. The initial deformed shape varied between a first and third mode buckling response throughout testing, but the final deformed shape exhibited a typical first mode buckling response for all replications. Typically the resulting experimental buckling load was higher for specimens demonstrating an initial third mode buckling response; this pattern led the author to consider the maximum buckling load resulting from an initial first mode buckling shape as the lower bound of the critical buckling load. Mottram considered the experimental buckling loads associated with an initial third mode buckling response to be the more accurate measure of the critical buckling load, therefore taking these values as the upper bound of a critical LTB load range (Mottram 1992).

Several models were compared with the experimental data found in Table 2-3, and each was plotted for $\frac{P_{c r} l^{2}}{4 \sqrt{E_{z, y y} I_{y y} G_{x y} J}}$ versus $\frac{l^{2} G_{x y} J}{E_{z, y y} I_{w}}$ as shown in Figure 2-4. The value of $\frac{l^{2} G_{x y} J}{E_{z, y y} I_{w}}$ shown along the $x$-axis indicates the overall contribution of warping to the torsional resistance of the specimen. In this figure, curves 1 through 3 represent the critical buckling resistance of a simplysupported beam for loading applied at the top flange, shear center, and bottom flange, respectively, calculated using Equation (2-33). Curve 4 is generated using Equation (2-34) where the ends of the beam are fully fixed from warping and rotation about the minor $y-y$ axis. As evidenced by the plot in Figure 2-4, the buckling resistance it dramatically increased for fixed conditions at the end supports (Mottram 1992). Curve 5 comes from a theoretical solution obtained by Timoshenko and Gere (1961) for warping and minor $y-y$ axis rotation restrained at the supports, and loading applied at the centroid of the section. Curve 6 is obtained by Mottram (1992) using a finite-difference approach for solving the fourth-order differential equation 
formulated by Timoshenko and Gere (1961) for loading applied at the shear center; curve 7 is obtained using this same approach for top flange loading. Lastly, curves 8 and 9 come from Eurocode 3 (1988) for fully fixed and simply-supported end conditions, respectively.

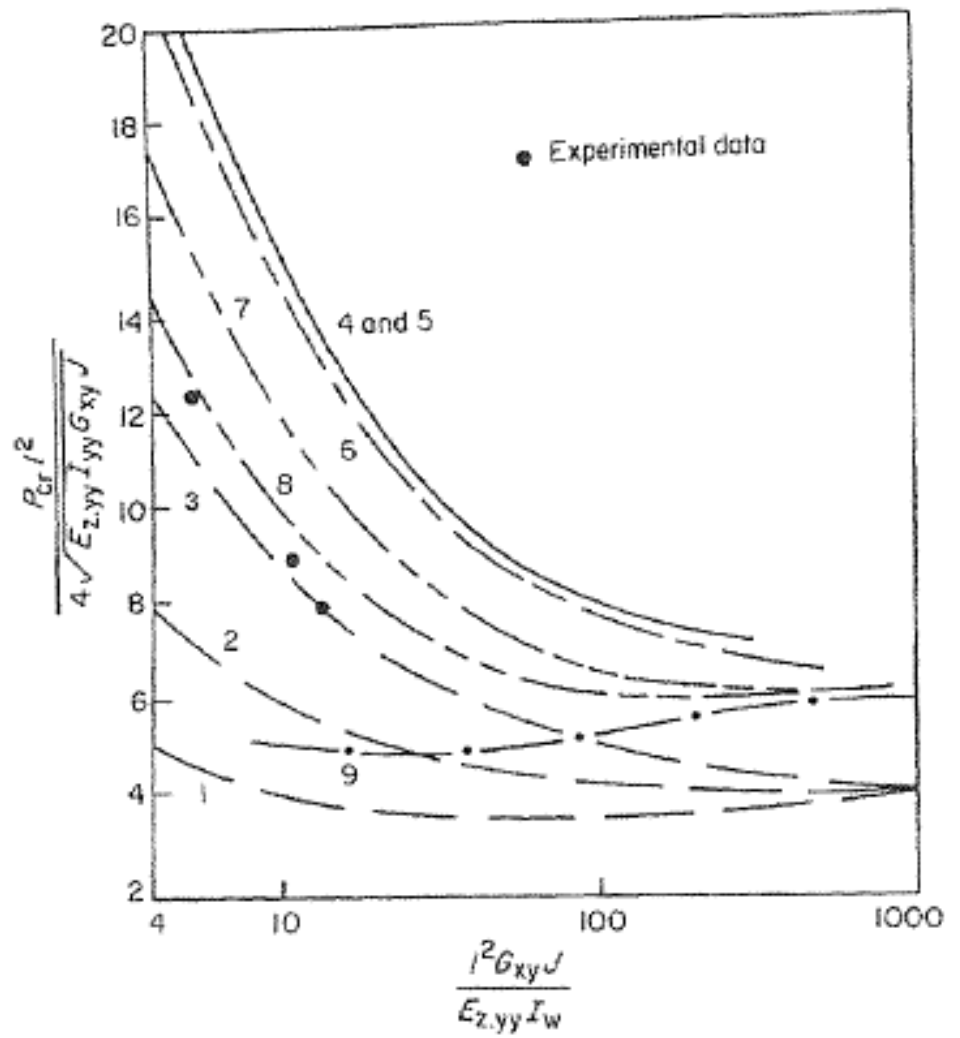

Figure 2-4 Critical loading for lateral-torsional buckling of simply-supported I-beams (Mottram 1992)

Because of the large difference in shear modulus values - $1.2 \mathrm{GPa}(0.17 \mathrm{Msi})$ from the fullscale testing and $3.0 \mathrm{GPa}(0.44 \mathrm{Msi})$ from the manufacturer - a parametric study was performed using three values of $G_{x y}$ within this range. The numerical results of this study can be found in Table 2-4. As seen in the table, even when using the minimum design values for $G_{x y}$ provided by the manufacturer of $1.2 \mathrm{GPa}(0.17 \mathrm{Msi})$, the results of each prediction model, with the exception of Eurocode 3 (1988) for top flange loading and free rotation at the end supports, vastly 
overestimated the maximum experimental critical LTB load. Taking the minimum design value for the shear modulus, the resulting error using Equation (2-34) is calculated as 14.6 percent.

Table 2-4 Theoretical predictions of critical loading for pultruded profile I102/51/6 at a span of $1500 \mathrm{~mm}$ (modified from Mottram 1992)

\begin{tabular}{|c|c|c|c|c|}
\hline \multirow{2}{*}{ Analysis } & \multirow[b]{2}{*}{$G_{x y}(G P a)$} & \multicolumn{3}{|c|}{$P_{\mathrm{cr}}(\mathrm{kN})$} \\
\hline & & 1.2 & 2.3 & 3.0 \\
\hline $\begin{array}{l}\text { Finite Difference, } \\
\text { (10 elements), } \\
\text { Figure 2-4 Curve } 7, \\
\text { top flange loading }\end{array}$ & & 6.59 & 7.43 & 7.71 \\
\hline $\begin{array}{l}\text { Finite Difference, } \\
\text { (10 elements), } \\
\text { Figure } 2-4 \text { Curve } 6, \\
\text { shear center loading }\end{array}$ & & 8.60 & 9.10 & 9.40 \\
\hline $\begin{array}{l}\text { MMFG Design } \\
\text { Manual, } \\
\text { Equation (2-34), } \\
\text { Figure 2-4 Curve 4, } \\
\text { shear center loading, } \\
k=k_{w}=0.5\end{array}$ & & 8.90 & 9.40 & 9.55 \\
\hline $\begin{array}{l}\text { Eurocode } 3, \\
\text { Figure } 2-4 \text { Curve } 9, \\
\text { top flange loading, } \\
k=0.5, k_{w}=1.0\end{array}$ & & 2.62 & 3.29 & 3.69 \\
\hline $\begin{array}{l}\text { Eurocode } 3, \\
\text { bottom flange loading, } \\
k=k_{w}=0.5\end{array}$ & & 10.46 & 11.13 & 11.53 \\
\hline
\end{tabular}

One cause of this error is the lack of full fixity at the end supports with respect to warping as well as rotation about the $y-y$ axis. Another major cause of error is failure to account for shear deformation in the prediction equation. Results of the experimental data suggest that as much as 15 percent of the total deflection was due to shear. 
A second early study of lateral-torsional buckling of pultruded I-sections worth noting was performed by Barbero and Raftoyiannis (1994). This study investigated the effects of crosssectional geometry on the overall failure mode of these types of specimens, including local buckling, lateral-torsional buckling, as well as a combined type of failure known as distortional buckling. Due to low stiffness in the transverse direction, pultruded open sections of certain dimensions are susceptible to this type of coupled failure which can result in a drastic reduction in the overall buckling capacity of the member (Barbero and Raftoyiannis 1994). At the time of this study, very little research existed related to distortional buckling failures, especially for FRP composites.

By employing von-Karman non-linear strain equations in terms of displacements to model the kinematics of the system, the total potential energy for the orthotropic plate components can be expressed in terms of displacements. This, combined with the Classical Lamination Theory, allowed the authors to develop an expression for the second variation of the total potential energy of the pultruded beam, shown in Equation (2-35). The critical buckling condition occurs when the first term in this equation, $\delta^{2} V$, is equal to zero (Barbero and Raftoyiannis 1994).

$$
\delta^{2} V=\delta^{2} V_{w}+\delta^{2}\left(V_{f}^{b}\right)_{T, B}+\delta^{2}\left(V_{f}^{p}\right)_{T, B}
$$

In which

$\delta^{2} V_{w}=$ second variation of the total potential energy of the web

$\delta^{2}\left(V_{f}^{b}\right)_{T, B}=$ second variation of the total potential energy for the flanges under bending $\delta^{2}\left(V_{f}^{p}\right)_{T, B}=$ second variation of the total potential energy of the flanges

In developing this equation, several assumptions were made, including that the beam is under pure bending about the strong axis, there is no distortion in the flanges (i.e., no bending in the $\mathrm{z}$ - 
direction), and axial forces are only carried by the flanges (Barbero and Raftoyiannis 1994). For a full derivation of this equation, refer to the original document by Barbero and Raftoyiannis (1994).

A numerical solution to this problem was attempted using the Raylaigh-Ritz method, in which the displacement of the web was modelled using the typical lateral-buckling distortion of the web of a simply supported I-section subjected to transverse loading. From this, an eigenvalue problem of the order $p \times q$ is formed in which the critical buckling load can be obtained as a function of the prebuckling stress resultants of the web $\left(N_{x}^{w}, N_{y}^{w}, N_{x y}^{w}\right)$ and flanges $\left(N_{x}^{f}\right)$, respectively (Barbero and Raftoyiannis 1994).

From this formulation, a study of the effect of ratios of various cross-sectional geometric properties as well as the span length on the overall buckling failure mode can be performed. For this study, Barbero and Raftoyiannis (1994) considered a total of five different size I-sections: $100 \mathrm{~mm} \times 100 \mathrm{~mm} \times 6.35 \mathrm{~mm}(3.94$ in. $\times 3.94$ in. $\times 0.25$ in. $), 150 \mathrm{~mm} \times 150 \mathrm{~mm} \times 6.35 \mathrm{~mm}$ (5.91 in. $\times 5.91$ in. $\times 0.25$ in. $), 150 \mathrm{~mm} \times 150 \mathrm{~mm} \times 9.53 \mathrm{~mm}(5.91$ in. $\times 5.91$ in. $\times 0.375$ in. $)$, $200 \mathrm{~mm} \times 200 \mathrm{~mm} \times 9.53 \mathrm{~mm}(7.87$ in. $\times 7.87$ in. $\times 0.375$ in. $)$, and $200 \mathrm{~mm} \times 100 \mathrm{~mm} \times 9.53$ $\mathrm{mm}(7.87$ in. $\times 3.94$ in. $\times 0.375$ in.). These specimens were examined at a number of test spans. A resulting plot of the critical load ratio of the critical lateral-distortional buckling load-to-flange local buckling load $\left(P_{o} / P_{L}\right)$ versus the ratio of the span length-to-radius of gyration $(l / r)$ is shown in Figure 2-5. From this plot, it can be seen that at higher ratios of $l / r$ (i.e., longer spans), the governing failure mode is lateral-torsional buckling, and at shorter spans, the failure mode is controlled by distortional buckling (Barbero and Raftoyiannis 1994). Similarly, two plots of the critical load ratio versus the height-to-width ratio were generated. The plot shown in Figure 2-6(a) exhibits a fixed depth, with a varying width; conversely, Figure 2-6(b) shows was created 
using a fixed width, with a varying member height. From these plots, it can be concluded that at high depth-to-width ratios, the governing mode of failure is most likely to be caused by LTB; meanwhile, specimens having a low depth-to-width ratio are controlled by lateral-distortional buckling.

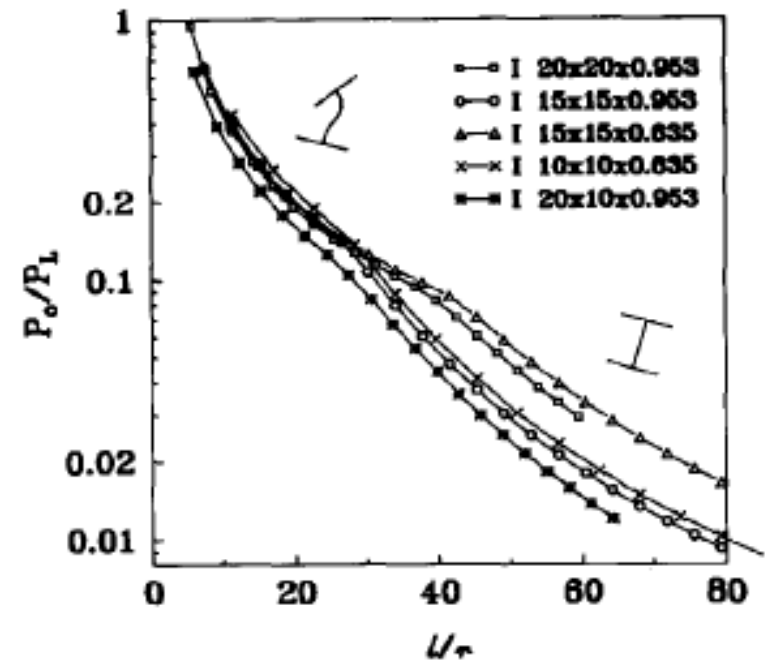

Figure 2-5 Critical load ratio $P_{o} / P_{L}$ versus ratio $l / r$ for load applied at the centroid (Barbero and Raftoyiannis 1994)

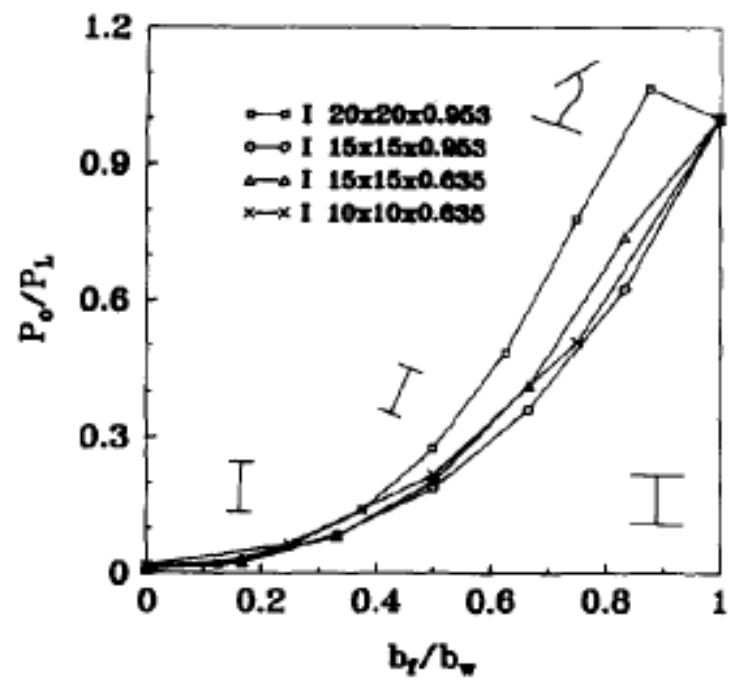

(a)

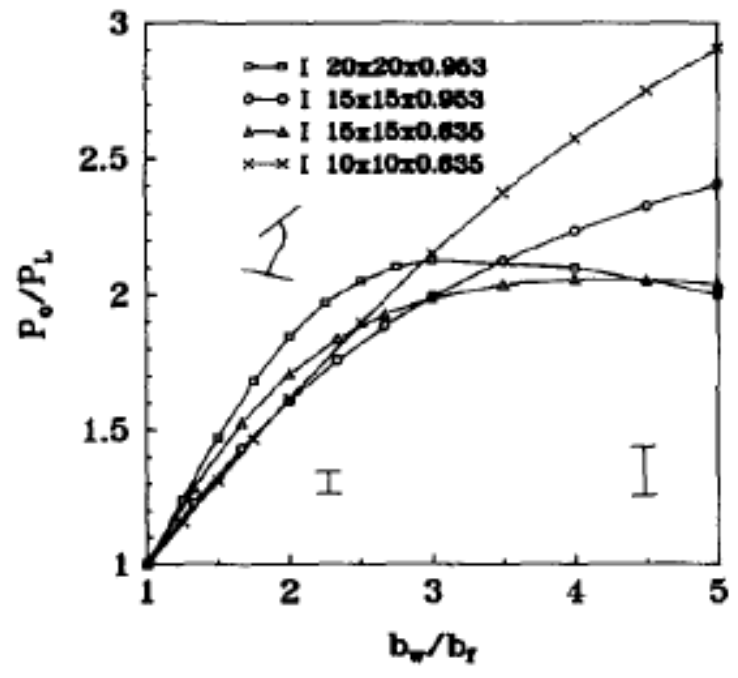

(b)

Figure 2-6 Critical load ratio $P_{o} / P_{L}$ versus ratio $b_{w} / b_{f}$ for load applied at the centroid generated by (a) using a fixed web depth $b_{w}$ and (b) using a fixed width $b_{f}$ (Barbero and Raftoyiannis 1994) 
Further evaluation of both lateral-torsional buckling and lateral-distortional buckling was carried out in a doctoral dissertation by Qiao (1997). In this study, design equations were derived for the prediction of both LTB and distortional buckling failures in a similar manner to Barbero and Raftoyiannis (1994), through the use of energy principles and nonlinear elastic plate theory. Due to the complexity and impractical nature of the distortional buckling equation which requires the use of computer software for a solution and does not fall under the scope of this report, only the LTB equation and results will be discussed herein. By expanding on the original derivation, a direct equation can be arrived at for the prediction of the critical LTB load capacity. In deriving this equation, the cross-section of the beam was assumed to remain straight (i.e., no distortion), therefore the lateral displacement components can be coupled. There resulting design formula for LTB is shown in Equation (2-36) (Qiao 1997).

$$
P_{c r}=\frac{2 \pi^{3} h \sqrt{\left(6 D_{11}+a_{11} h^{2}\right)\left[(\pi h)^{2}\left(2 D_{11}+4 d_{11}+a_{11} h^{2}\right)+48\left(2 D_{66}+d_{66}\right) L^{2}\right]}}{3\left(\pi^{2}+4\right) L^{3}}
$$

In which

$h=$ height and width of the beam (assumed to be equal in this study)

$D_{11}, D_{66}=$ values from the bending stiffness matrix $\left[D_{i j}\right]$

$a_{11}=1 / \alpha_{11}$ where $\left[\alpha_{i j}\right]$ is the inverse of the stretching stiffness matrix $\left[A_{i j}\right]$

$d_{11}=1 / \delta_{11}$ where $\left[\delta_{i j}\right]$ is the inverse of the bending stiffness matrix $\left[D_{i j}\right]$

$d_{66}=1 / \delta_{66}$ where $\left[\delta_{i j}\right]$ is the inverse of the bending stiffness matrix $\left[D_{i j}\right]$

$L=$ span length

In this study, the values of the stretching stiffness matrix $\left[A_{i j}\right]$ and the bending stiffness matrix $\left[D_{i j}\right]$, as well as the material and section stiffness properties are measured using micro/macromechanics models (Qiao 2007). 
The accuracy of this equation was evaluated based on experimental testing performed on two $304.8 \mathrm{~mm} \times 304.8 \mathrm{~mm} \times 12.7 \mathrm{~mm}(12$ in. $\times 12 \mathrm{in} . \times 0.5$ in. $)$ pultruded WF sections with two different fiber architectures. The first specimen consisted of rovings, continuous strand mats (CSM), and \pm 45 degree angle-ply stitched fabrics. The second specimen had this same fiber layup with the addition of 0/90 degree stitched fabric layers. Both specimens were tested under three-point bending at a span of $4.42 \mathrm{~m}(14.5 \mathrm{ft})$. Lateral restraint was provided at the end supports by preventing rotation of the flanges from the top and bottom of the specimen; the lateral restraining mechanism did not touch the outer edges of the flanges, which is a more popular approach. The ends of the beam were allowed to rotate freely along the major axis. Wooden stiffeners were fit between the flanges and web at midspan in order to induce a LTB failure (Qiao 1997).

In order to capture the initiation of LTB, transverse bars were attached to both the tension and compression flanges, so that LVDTs could measure the angle of rotation during testing; an additional LVDT was provided to record the midspan vertical deflection directly under the load. Linear strain gages were installed on the outer ends of the compression flange to detect the point of buckling instability at these locations. The loading ram was applied to a steel spacer beam which distributed the load across the width top flange, reducing the possibility of localized crushing. Plots of the load versus displacement and load versus strain in the flanges were generated, and the critical buckling load was determined by the point of slope change in these diagrams (Qiao 1997). Further verification of this model was attempted using an FE model created using the ANSYS software package. The test specimens were modelled using 8-node isoparametric layered shell elements. Further details of the constructed FE model are discussed in Qiao's dissertation (1997). 
The design equation was varied to assume the load was applied at three different locations: at the centroid, halfway between the centroid and the top flange, and at the top flange. When assuming the load to be applied halfway between the centroid and top flange the resulting error compared with the average experimental value was -6.25 percent for the first specimen type, and -9.98 percent for the second specimen which included 0/90 degree fabric layers. This type of loading also resulted in good correlation with the finite element model. However, when the loading was assumed to be applied at the top of the compression flange, which is the case in structural engineering applications, the resulting errors grew to +23.4 percent and +18.0 percent, respectively (Qiao 1997).

Another approach to developing an analytical model for calculating the critical LTB load capacity of pultruded FRP open sections was performed by Ascione et al. (2011). This model is modified from the work of Vlasov (1961) related to thin-walled isotropic beams. The original Vlasov model is only applicable when the influence of shear deformation is negligible, which is rarely the case for pultruded sections. By assuming the total torsional rotation to be comprised of two main components - one associated with axial warping and the other with shear strain - to be accounted for using Timoshenko's beam theory, a model can be developed which considers the effect of shear strain combined with the Vlasov model (Ascione et al. 2011). This model serves as an expansion on previous work performed by Roberts and Al-Ubaidi (2002). The result of this derivation for the case of a thin-walled I-section can be written in expanded form, from which a system of differential equations can be developed for predicting the buckling mode as well as calculating the critical LTB load capacity. This is solvable using a finite element approximation using a two-node one-dimensional element, with the kinematic unknowns approximated using Hermite cubic interpolation functions (Ascione et al. 2011). The results of this analytical model 
are compared to results obtained by Roberts (2002) from three different size I-sections for two loading cases: (1) two equal applied end moments and (2) uniformly distributed load along the top flange of the beam along the centroidal $x$-axis. The results of the model proposed by Ascione et al. (2011) are found to be more conservative than those calculated using the Roberts (2002) model for both load cases. Furthermore, a parametric study was used to conclude that as the ratio of the longitudinal elastic modulus-to-in-plane shear modulus $\left(E_{z} / G_{t z}\right)$ increases, the influence of shear deformation also increases (Ascione et al. 2011). Neither of the models considered in this study could be considered practical for use in a design scenario.

A final study used for discussion in this report is based on some of the most recent research of LTB of pultruded profiles carried out by Nguyen et al. (2014). Similar to the aim of this report, the scope of the study by Nguyen et al. was to collect data from a large number of tests from various cross-sections at numerous test spans under multiple loading conditions, and develop simplistic formulae suitable for use in a design code. As attempted by other researchers, Nguyen et al. began with a widely accepted design formula for isotropic steel open sections, shown in Equation (2-37) (Clark and Hill 1960), and attempted to make appropriate substitutions of orthotropic elastic constants for use in the design of pultruded FRP members.

$$
M_{c r}=C_{1} \frac{\pi^{2} E I_{z}}{(k L)^{2}}\left(\sqrt{\left(\frac{k}{k_{w}}\right)^{2} \frac{I_{w}}{I_{z}}+\frac{(k L)^{2} G I_{T}}{\pi^{2} E I_{z}}+\left(C_{2} z_{g}\right)^{2}}-C_{2} z_{g}\right)
$$

In which

$M_{c r}=$ elastic critical buckling moment of resistance

$C_{1}=$ factor to account for the type of moment distribution and support conditions

$C_{2}=$ factor to account for the vertical position of the load with respect to the shear center $E=$ modulus of elasticity 
$G=$ shear modulus

$I_{w}=$ warping rigidity

$I_{z}=$ second moment of area for flexure about the beam's minor axis

$I_{T}=$ torsional rigidity

$k=$ restraint factor for lateral flexural bending at the end supports

$k_{w}=$ restraint factor for warping at end supports

$L=$ span length

$z_{g}=$ vertical distance from the shear center to the point of load application

The values of $k$ and $k_{w}$ both vary from 0.5 for complete fixity to 1.0 for unrestrained. The values of $C_{1}$ and $C_{2}$ are dependent on the displacement boundary conditions (Nguyen et al. 2014).

There are two distinct combinations of end support conditions evaluated in this study. The first, which will be referred to as EC1 for simplicity, is a simply supported beam subjected to three-point bending; the second, which likewise will be referred to as EC2, provided lateral restraint at the end supports. Thus, for EC1, the value of both $k$ and $k_{w}$ will be 1.0, and for EC2 $k$ is equal to 0.5 and $k_{w}$ will be taken as 0.5 . Although an investigation of the values of $C_{1}$ and $C_{2}$ by the authors yield contradictory results, the most recently available values were used. Therefore, for EC1, the values of $C_{1}$ and $C_{2}$ are taken as 1.348 and 0.630 , respectively. For the second end support condition, EC2, the values of $C_{1}$ and $C_{2}$ will be 1.07 and 0.432 . These chosen values were originally developed for thin-walled cross-sections of isotropic material, and therefore may not be valid for pultruded profiles (Nguyen et al. 2014). By substituting in the values of these constants, two distinct equations for predicting the critical buckling capacity of pultruded members based on the end support conditions are developed. The first, shown in 
Equation (2-38), applies to a simply supported member; the second, shown in Equation (2-39), is valid for a specimen where lateral movement if fully fixed at the ends (Nguyen et al. 2014).

$$
\begin{gathered}
P_{c r}=\frac{4 M_{c r}}{L}=\frac{5.39 \pi^{2} E_{L} I_{z}}{L^{3}}\left(\sqrt{\frac{I_{w}}{I_{z}}+\frac{L^{2} G_{L T} I_{T}}{\pi^{2} E_{L} I_{z}}+0.40 z_{g}^{2}}-0.63 z_{g}\right) \\
P_{c r}=\frac{8.56 \pi^{2} E_{L} I_{z}}{L^{3}}\left(\sqrt{\frac{I_{w}}{I_{z}}+\frac{L^{2} G_{L T} I_{T}}{\pi^{2} E_{L} I_{z}}+0.75 z_{g}^{2}}-0.86 z_{g}\right)
\end{gathered}
$$

In which

$P_{c r}=$ elastic critical buckling load

$M_{c r}=$ elastic critical buckling moment of resistance

$E_{L}=$ longitudinal modulus of elasticity

$G_{L T}=$ in-plane shear modulus

$I_{w}=$ warping rigidity

$I_{z}=$ second moment of area for flexure about the beam's minor axis

$I_{T}=$ torsional rigidity

$L=$ span length

$z_{g}=$ vertical distance from the shear center to the point of load application

The values $E_{L}=23 \mathrm{kN} / \mathrm{mm}^{2}$ and $G_{L T}=3 \mathrm{kN} / \mathrm{mm}^{2}$ were provided by the manufacturer for use in the prediction equations.

The accuracy of these equations was tested by comparing the predicted values versus experimental data from 114 tests on four different size specimens: one I-section measuring 120 $\mathrm{mm} \times 60 \mathrm{~mm} \times 6 \mathrm{~mm}(4.72 \mathrm{in} . \times 2.36 \mathrm{in} . \times 0.24$ in. $)$ and three channel specimens measuring 120 $\mathrm{mm} \times 50 \mathrm{~mm} \times 6 \mathrm{~mm}(4.72$ in. $\times 1.97$ in. $\times 0.24$ in. $), 100 \mathrm{~mm} \times 50 \mathrm{~mm} \times 6 \mathrm{~mm}(3.94$ in. $\times 1.97$ 
in. $\times 0.24$ in.), and $100 \mathrm{~mm} \times 30 \mathrm{~mm} \times 6 \mathrm{~mm}(3.94$ in. $\times 1.18$ in. $\times 0.24$ in.), respectively. With the exception of the smallest channel specimen which was not tested at the longest span, each section size was tested at five span lengths ranging from $1828 \mathrm{~mm}$ (72 in.) to $4064 \mathrm{~mm}$ (160 in.). Each specimen was tested under both end conditions (EC1 and EC2), with the load height applied at three distinct positions: at the top flange, at the shear center, and at the bottom flange. In order to achieve this, the test set-up consisted of a steel disc mounted at midspan which acts as a pulley from which a steel cable is hung to transfer the load in the vertical direction. The pulley is equipped with a ball bearing such that loading will remain vertical with the point of load application remaining consistent with the center of the steel disc, even as LTB begins to initiate. Therefore, different loading heights may be achieved by repositioning the center of the steel disc along the depth of the test specimen (Nguyen et al. 2014). The load was applied by manually attaching dead weights to the load hanger, up to $2 \mathrm{kN}$ (450 lbf). When the load necessary to cause buckling exceeded $2 \mathrm{kN}$ (450 lbf), a hydraulic tension jack was used to pull on the steel cables. Steel angles were fixed $20 \mathrm{~mm}(0.79 \mathrm{in}$.) to each side of the test specimen. When the specimen had rotated enough to make contact with either of these angles, the test was concluded. During testing, the rotation at midspan was continuously recorded using an inclinometer attached to the steel loading disc. Vertical and horizontal deflection was also recorded using $50 \mathrm{~mm}$ strain gage displacement transducers. The resulting data was used in determining the load at which LTB occurs. Figure 2-7 illustrates the load configuration (Nguyen et al. 2014). 


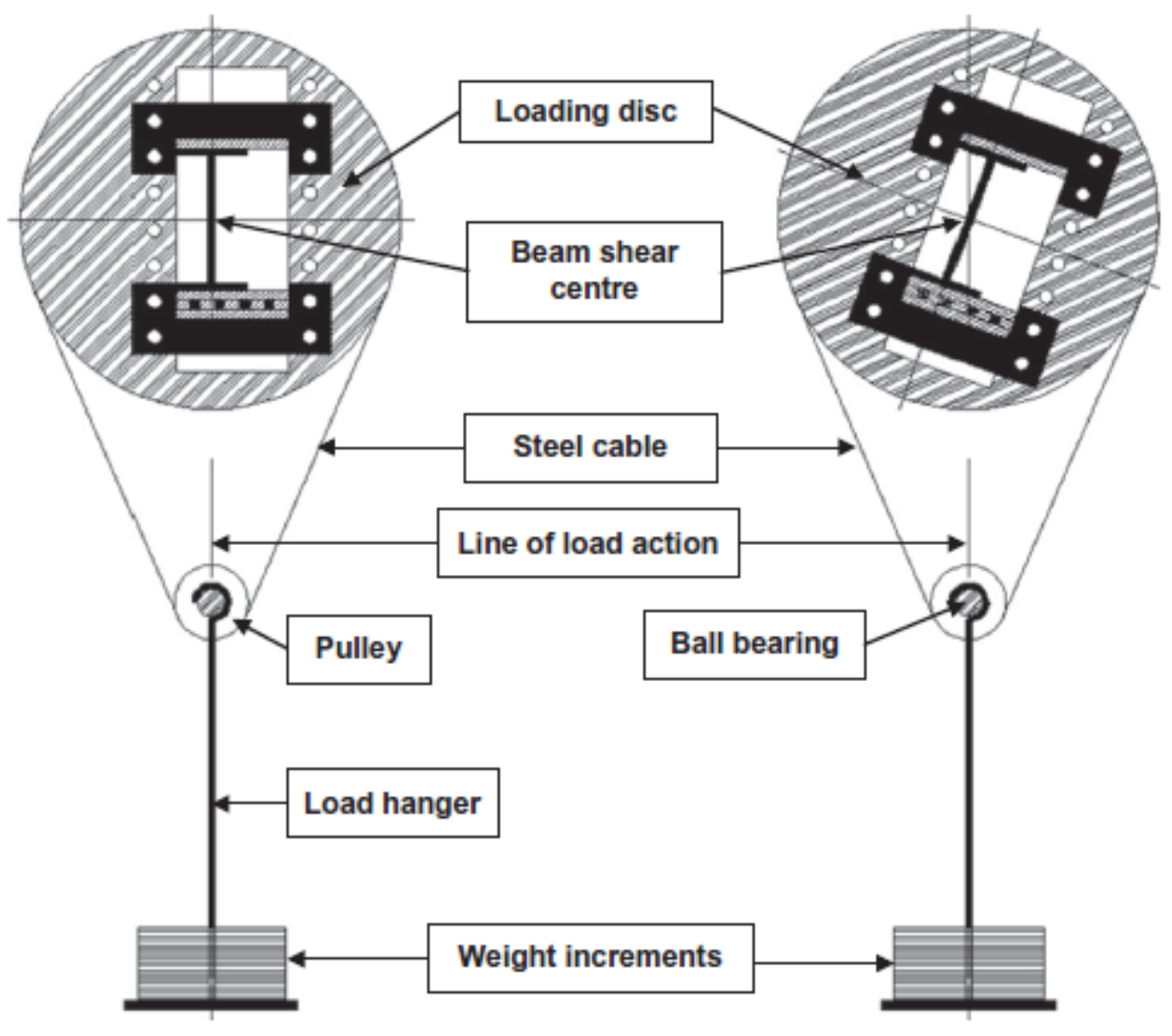

(a)

(b)

Figure 2-7 Diagram of loading disc system (a) prior to LTB failure and (b) after LTB failure (Nguyen et al. 2014)

According to theories of elastic stability, a LTB failure should occur suddenly when the critical buckling capacity is reached. This type of failure is known as 'bifurcation buckling' (Timoshenko and Gere 1961). This type of failure can only exist under ideal conditions where the beam is perfectly straight, homogeneous, and the loading is applied directly through the shear center (Nguyen et al. 2014). Therefore, it is rare to observe this type of failure in real engineering applications, particularly with the use of pultruded FRPs which, by nature, are inhomogeneous. A more common type of LTB failure is characterized by a gradual twisting and deformation as the applied load increases, making it difficult to pinpoint an exact critical buckling load (Nguyen 
et al. 2014). During this study, a select number of test specimens exhibited a failure similar to 'bifurcation buckling,' although the more gradual LTB failure was more prevalent.

The results of the prediction model given by Equations (2-38) and (2-39) were compared to 57 tests performed under EC1, and an additional 57 performed using EC2. When buckling occurred instantaneously, the peak load was taken to be the critical LTB load value. When lateral deformation occurred more gradually, the critical LTB load was determined using the Southwell plot method, where the critical LTB load level is equal to the gradient of the plot of $\varphi P$ versus $\varphi$, where $\varphi$ denotes the rotation of the steel loading disc (Southwell 1932).

The results provided by Nguyen et al. (2014) show that the analytical results were always significantly lower than the experimental results. For the lone I-section included in this study, under simply-supported end conditions, the percent error ranged from 70.7 to 92.9 percent for loading applied to the top flange. Similarly, the percent difference ranged from 63.6 to 74.9 percent for shear center loading, and 49.7 to 65.2 percent for loading at the bottom flange. The results were similar for the three channel specimens. Taking into account all three channels tested under EC1, the percent error ranged from 33.3 to 102.5 percent for loading applied to the top flange, 26.7 to 80.0 percent for shear center loading, and 20.8 to 64.9 percent for loading at the bottom flange. Under restrained end conditions (EC2), results were inconsistent, although somewhat improved, particularly for loading at the bottom flange. For the I-section, the percent error ranged from 71.7 to 107.6 percent for loading applied to the top flange, 54.0 to 64.1 percent for shear center loading, and 29.3 to 44.1 percent for loading at the bottom flange. As for the channel specimens, the error percentage for all three samples ranged from 35.6 to 104.1 percent for loading applied to the top flange, 27.4 to 66.2 percent for shear center loading, and 4.6 to 58.6 percent for loading at the bottom flange. It should be noted that the highest error 
percentages occurred for top flange loading, which is the most likely load scenario for structural engineering applications.

From the experimental data, Nguyen et al. (2014) concluded that both the effect of the load height as well as changing end conditions has a dramatic effect on the buckling load, where buckling resistance was increased under fixed end conditions. The data also suggests that the influence of warping restraint at the end supports has a significant influence on buckling behavior. The authors believe that the predicted values were always lower than the experimental buckling loads due to conservative elastic property values provided by the manufacturer. It is also believed that the values of $C_{2}$ must be modified for FRP composite sections. Additional sources of error proclaimed by the author include the presence of initial geometric imperfections in the pultruded members, and also an inability to fully satisfy the theoretical boundary conditions at the end supports (Nguyen et al. 2014). While Nguyen et al. (2014) believe that the conservative nature of these closed-form equations make them potentially suitable for a design standard, they suggest that accounting for geometric imperfections, shear deformation, viscoelasticity, and the influence of local buckling mechanisms in a revised form of these equations could result in improved accuracy and consistency. 


\section{CHAPTER 3 BOX BEAM FAILURE PREDICTION}

\subsection{Introduction and Scope}

When compared with experimental data from three-point bend testing conducted at the West Virginia University Constructed Facilities Center, the current model found in Chapter 5 of the most recently publicly available ASCE/ACMA Pre-Standard for Load and Resistance Factor Design (LRFD) of Pultruded Fiber Reinforced Polymer (FRP) Structures, the proposed equations for the design of members in flexure and shear were found to grossly over-predict the failure load capacity by as much as a factor of four (Qureshi 2012). The failure modes proposed within the chapter include: material rupture, local instability, lateral-torsional buckling, web shear buckling, tensile rupture of the web, web crippling, web compression buckling, and flange flexural failure. While the other failure modes may exist under certain geometric conditions of thin-walled FRP box shapes, it was determined by the author through extensive experimental and theoretical evaluation that there are only three primary governing modes of failure controlling the behavior of pultruded GFRP box members in flexure and shear under transverse loading.

The objective of the testing was to relate the failure mode to threshold values based on span length and/or cross-sectional dimensions. It was hypothesized prior to testing that the changes in failure mode could be related to previously undetermined ratios of beam span-to-depth $(L / h)$, flange width-to-thickness $\left(b_{f} / t_{f}\right)$, and web depth-to-thickness $\left(d_{w} / t_{w}\right)$. Therefore, the test matrix was designed to covered several variations in beam sizes and test spans during testing, and evaluated the data for a wide range of potential failure modes. 


\subsection{Samples and Test Procedure}

All of the beam specimens tested and evaluated for static load cases in this study were pultruded square or rectangular GFRP box sections, with depths and widths ranging from 3 inches to 6 inches, and wall thicknesses ranging from $1 / 4$ inch to $3 / 8$ inch. The beams were constructed from E-glass fibers and mats, and also some with fabrics, having a modulus of elasticity, $E_{f}$, of $\sim 10.4$ Msi. Similarly, vinyl ester or polyester resin, with a modulus of elasticity, $E_{m}$, of $\sim 0.49$ Msi was used as a binder. Three-point bending tests to failure were performed on the samples over spans ranging from 18 inches to 180 inches. A list of those sections included in the study can be seen in Table 3-1, with the dimensions shown in the table explained by Figure 3-1.

Table 3-1 Summary of samples tested for analysis of square and rectangular box sections

\begin{tabular}{|c|c|c|c|c|c|c|}
\hline Sample & $\mathbf{t}$ (in.) & $\mathbf{b}_{\mathrm{f}}$ (in.) & $\mathbf{b}_{\mathbf{c}}$ (in.) & $\mathbf{h}$ (in.) & $\mathbf{d}_{\mathrm{w}}$ (in.) & Area (in. $\left.{ }^{2}\right)$ \\
\hline $3^{\prime \prime} \times 3^{\prime \prime} \times 0.25^{\prime \prime}$ & 0.25 & 3 & 2.5 & 3 & 2.5 & 2.75 \\
\hline $3.5^{\prime \prime} \times 3.5^{\prime \prime} \times 0.25^{\prime \prime}$ & 0.25 & 3.5 & 3 & 3.5 & 3 & 3.25 \\
\hline $4^{\prime \prime} \times 4^{\prime \prime} \times 0.375^{\prime \prime}$ & 0.375 & 4 & 3.25 & 4 & 3.25 & 5.44 \\
\hline $6 " \times 4 " \times 0.25 "$ & 0.25 & 4 & 3.5 & 6 & 5.5 & 4.75 \\
\hline $6^{\prime \prime} \times 6 " \times 0.375^{\prime \prime}$ & 0.375 & 6 & 5.25 & 6 & 5.25 & 8.44 \\
\hline
\end{tabular}

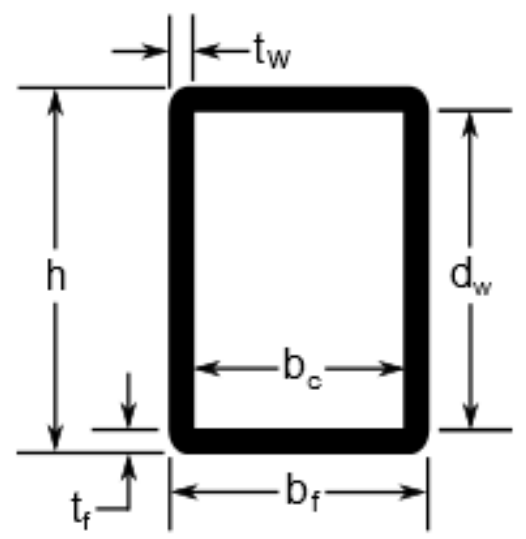

Figure 3-1 Dimensions for use in proposed model for square and rectangular box sections 
As will be seen in Section 3.3, the proposed model for predicting the critical flexural load capacity of closed GFRP full-size sections is largely dependent on laminate properties and the total number of laminae. These characteristic material properties include the longitudinal elastic modulus of both the web and the flange, the transverse elastic modulus of the web, the in-plane shear modulus, Poisson's ratio, and longitudinal strength in the flange. Due to the high cost of full-size member testing, these properties are often obtained by coupon level testing. In this study, tests are performed on full-size sections up to spans of 180 inches, and also on coupons to establish data correlations with the proposed theory. Because the failure modes most often exhibited in these types of members were occurred in the tension flange or tension zone of the web, the elastic moduli used in the proposed model were obtained from coupon level tension testing as discussed in Appendix C. The values for each section are shown in Table 3-2.

Table 3-2 Laminate properties for box sections included in this study based on coupon level tension testing

\begin{tabular}{|c|c|c|c|c|c|c|c|}
\hline Sample & $\mathbf{E}_{\mathrm{L}, \mathrm{f}}$ (Msi) & $\mathbf{E}_{\mathrm{L}, \mathrm{w}}(\mathbf{M s i})$ & $\mathbf{E}_{\mathrm{T}, \mathrm{f}}(\mathbf{M s i})$ & $\mathbf{E}_{\mathrm{T}, \mathrm{w}}(\mathbf{M s i})$ & $\mathbf{G}_{\mathrm{LT}}(\mathbf{M s i})$ & $v_{L T}$ & $F_{\text {Lf }}(\mathbf{k s i})$ \\
\hline $3^{\prime \prime} \times 3^{\prime \prime} \times 0.25^{\prime \prime}$ & 5.12 & 5.12 & 1.548 & 1.548 & 0.50 & 0.43 & 48.7 \\
\hline $3.5^{\prime \prime} \times 3.5^{\prime \prime} \times 0.25^{\prime \prime *}$ & 4.30 & 4.30 & 1.300 & 1.300 & 0.42 & 0.33 & 40.0 \\
\hline $4^{\prime \prime} \times 4 " \times 0.375^{\prime \prime *}$ & 4.30 & 4.30 & 1.300 & 1.300 & 0.42 & 0.33 & 40.0 \\
\hline $6 " \times 4^{\prime \prime} \times 0.25 " *$ & 4.30 & 4.30 & 1.300 & 1.300 & 0.42 & 0.33 & 40.0 \\
\hline $6 " \times 6 " \times 0.375^{\prime \prime *}$ & 4.30 & 4.30 & 1.300 & 1.300 & 0.42 & 0.33 & 40.0 \\
\hline
\end{tabular}

*denotes typical laminate property values based on data shown by Qureshi (2012)

While some of these values were obtained through coupon level testing performed by the author, shown in Appendix C, many of the values shown are typical values based on longitudinal, transverse, and shear elastic moduli obtained through static coupon tension tests performed by Qureshi (2012), as noted within the table. The shear modulus for the $3^{\prime \prime} \times 3^{\prime \prime} \times 0.25^{\prime \prime}$ box section is a generalized value based on a similar ratio of $E / G$ observed in data from Qureshi 
(2012). It should also be noted that this study assumes elastic modulus values in the flange do not differ from those in the web in the longitudinal direction (along the span) of a specimen. However, average values transverse to the longitudinal direction were determined using coupon tests, as shown in the data presented in Appendix C.

For all tests, the support conditions were steel cylinder roller-type supports under the ends, which varied in diameter depending on the test span, as detailed in Sections 3.2.1 through 3.2.3. The load was applied at the center of the span by a hydraulic actuator. Strain gages having a $1 / 4$ inch gage length were installed on the tension flange at midspan and mid-width of each specimen to record longitudinal bending strain. Additional strain gages were installed at a 40 degree angle within the tension zone of the web on a select number of $3 " \times 3 " \times 0.25$ " samples tested in the range of $6 \leq L / h \leq 10$ in order to confirm the principal strain range, as further discussed in Section 3.3.2. All specimens were tested to rupture. It should be noted that currently no ASTM standard exists for this type of testing.

\subsubsection{Test Procedure for Spans Less than 20 inches}

For test spans less than 20 inches, specimens were tested using an Instron System 8500 Series Servohydraulic Testing Machine. This testing machine allows for the midspan vertical load to be applied at a constant head displacement rate of $0.2 \mathrm{in} . / \mathrm{min}$., while continuously recording the load and displacement. This information was then transferred continuously to a StrainSmart system utilizing a Vishay System 5000 data acquisition system. At the same time, the data acquisition system was being used to record strain levels at the locations discussed in Section 3.2. The data acquisition system allowed for all data to be recorded at the same rate, and exported into one comprehensive data spreadsheet. 
When using this machine, supports were steel cylinder roller-type supports having a diameter of $3 / 8$ inch. Because of the minimal contact area of the roller supports, a $1 / 8$ inch thick piece of ultra-strength neoprene rubber elastomeric bearing padding having approximate durometer hardness 30A was draped over the roller in order to prevent localized crushing at the supports. The load was distributed from the loading nose using a $1 / 4$ inch thick steel load distribution plate 3 inches in length along the test span. Under the steel load distribution was two $1 / 4$ inch thick pieces of ultra-strength neoprene rubber elastomeric bearing padding having durometer hardness 50A stacked one on top of the other for a total elastomeric pad thickness of $1 / 2$ inch. The area of the elastomeric padding exceeded the area of the steel load distribution plate. The added thickness of padding was used to protect against a localized punching failure. It should be noted that some tests were also performed using a GFRP load distribution plate for comparison, although no significant difference in results was observed. The loading plate and elastomeric padding was aligned with the centerline of the specimen during all tests. A photo of this test setup can be seen in Figure 3-2. 


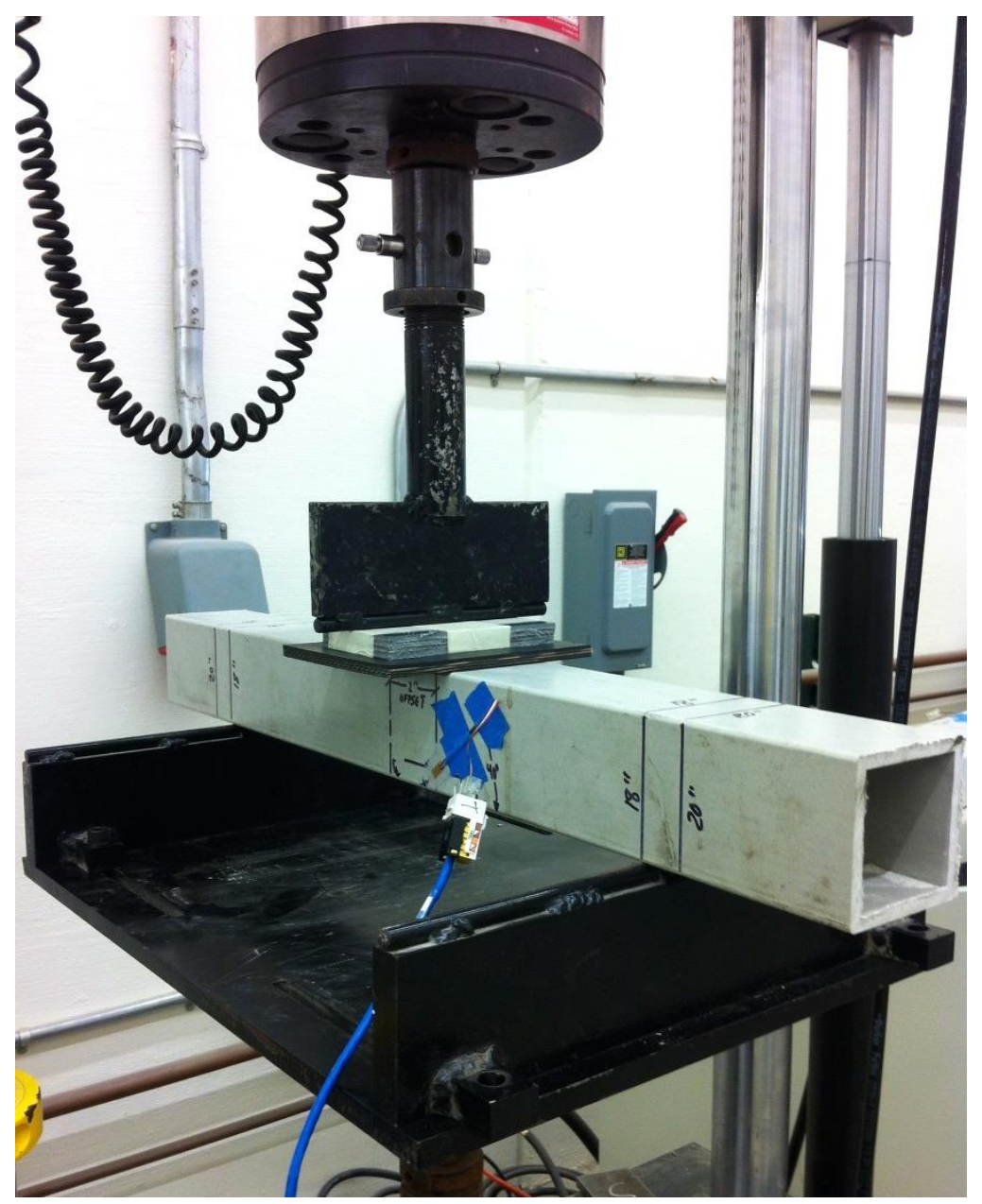

Figure 3-2 Setup using Instron System 8500 Series and GFRP load distributing plate for testing of a $3 " \times 3 " \times 0.25 "$ sample at a test span of 18 inches

\subsubsection{Test Procedure for Spans Ranging from 20 inches to 30 inches}

For test spans ranging from 20 inches to 30 inches, specimens were tested using an Instron Industrial Series 1000HDX Model Servohydraulic Testing Machine. This testing machine allowed for the midspan vertical load to be applied at a constant head displacement rate of $0.2 \mathrm{in.} / \mathrm{min}$., while continuously recording the load and displacement using the built-in Partner $^{\mathrm{TM}}$ Universal Materials Testing Software provided by Instron. This information was then transferred continuously to a StrainSmart system utilizing a Vishay System 5000 data acquisition system. At the same time, the data acquisition system was being used to record strain levels 
obtained by the strain gage at a location as discussed in Section 3.2. This allowed for all data to be recorded continuously at a rate of 10 recordings per second, and exported into one comprehensive data spreadsheet.

When using this machine, supports were steel cylinder roller-type supports having a diameter of 2 inches. The load was distributed from the loading nose using a 1/4 inch thick steel load distribution plate 3 inches in length along the test span. Under the steel load distribution was a $1 / 4$ inch thick piece of ultra-strength neoprene rubber elastomeric bearing padding having durometer hardness $50 \mathrm{~A}$ to protect against a localized punching failure. The area of the elastomeric padding exceeded the area of the steel load distribution plate. The loading plate and elastomeric padding was aligned with the centerline of the specimen during all tests. A photo of this test setup can be seen in Figure 3-3.

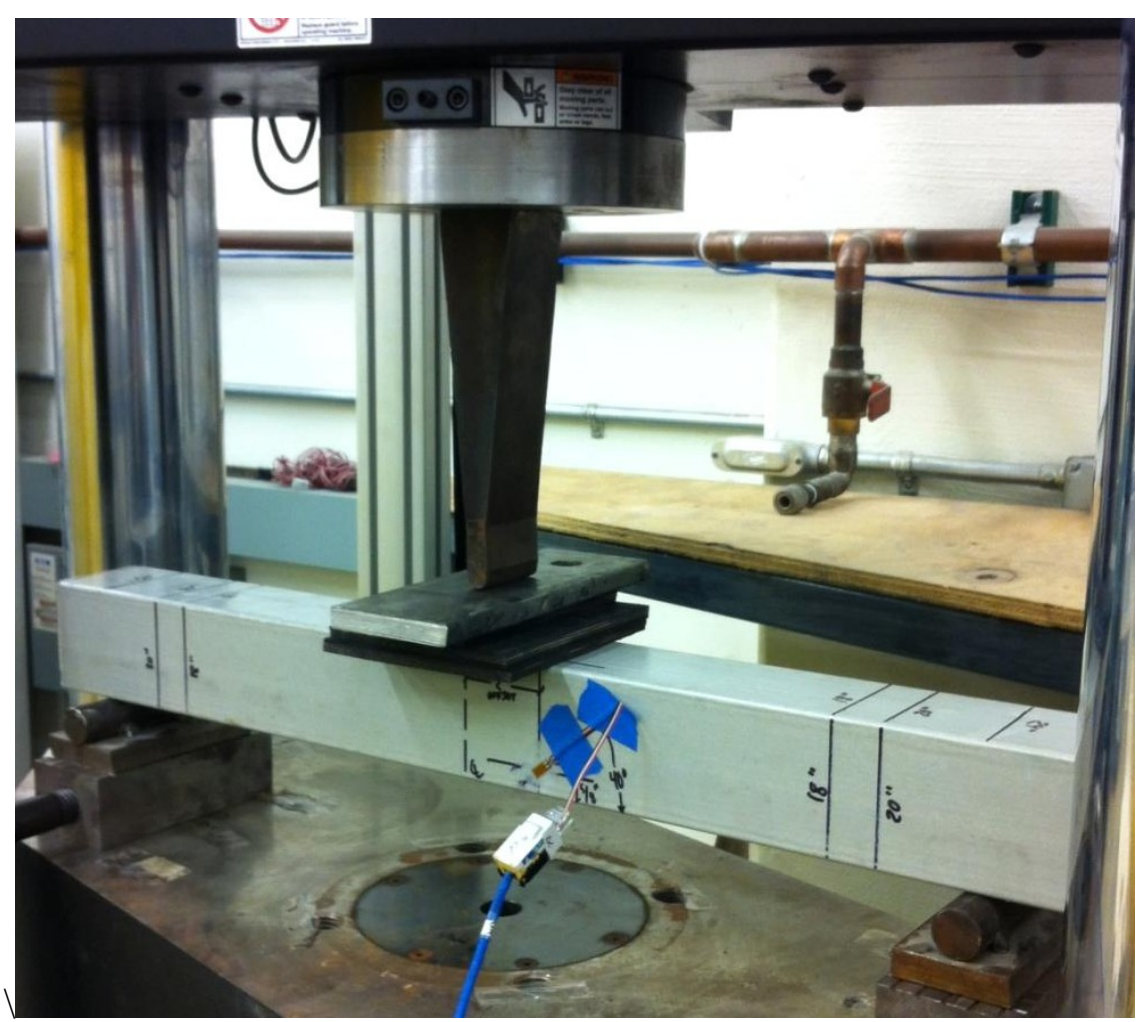

Figure 3-3 Setup using Instron Industrial Series 1000HDX Model and steel load distributing plate for testing of a $3 " \times 3 " \times 0.25 "$ sample at a test span of 24 inches 


\subsubsection{Test Procedure for Spans Greater than 30 inches}

For specimens tested at spans exceeding 30 inches, specimens rested on steel cylinder roller-type supports having a diameter of 4 inches. These steel rollers were placed on concrete balusters which allowed for adequate room for deflection measuring devices, and could be

moved to meet a variety of test span requirements. The midspan vertical load was applied manually using hydraulic actuator operated by a hand pump, and therefore loading rate could not be held constant throughout testing at spans greater than 30 inches. The load was recorded using a load cell. During testing of smaller sections where the expected flexural load capacity fell well below 10,000 lbf, a 10 kip load cell (Omega LC101-10K) was used. For testing of larger specimens where the expected flexural load capacity was near or above 10,000 lbf, a 40 kip load cell (Omega LC101-40K) was used.

The vertical deflection at midspan was continuously recorded on both walls of the test specimens using two linear variable displacement transducers (LVDTs) having a measurable range of \pm 3 inches (Schavitz/MS: $3000 \mathrm{HCD}$ ) which were placed under the test specimen. The strings connecting the two LVDTs to the test specimen were run through one pulley on each side, so as to prevent damage to the LVDTs at the point of rupture. All load, strain, and deflection data was continuously recorded using a StrainSmart computer program utilizing a Vishay System 5000 data acquisition system during each of the trials. The load was distributed from the loading nose using a $1 / 4$ inch thick steel load distribution plate 6 inches in length along the test span. Under the steel load distribution was a $1 / 4$ inch thick piece of ultra-strength neoprene rubber elastomeric bearing padding having durometer hardness $50 \mathrm{~A}$ to protect against a localized punching failure. The area of the elastomeric padding exceeded the area of the steel load distribution plate. The loading plate and elastomeric padding were aligned with the 
centerline of the specimen during all tests. In some instances, the load was distributed using a steel spacer column as can be seen in Figure 3-4, which was also about 6 inches in length along the test span. This spacer column was used to fill excess space during testing of samples having a small total depth, so that the stroke limit of the hydraulic actuator would not be exceeded. It should be noted that some small variations in the loading plate length existed throughout testing in this span range. A photo of this test setup can be seen in Figure 3-4, where the 40 kip load cell can be seen on top of the spacer column, and the LVDTs are clearly visible near the bottom of the photo.

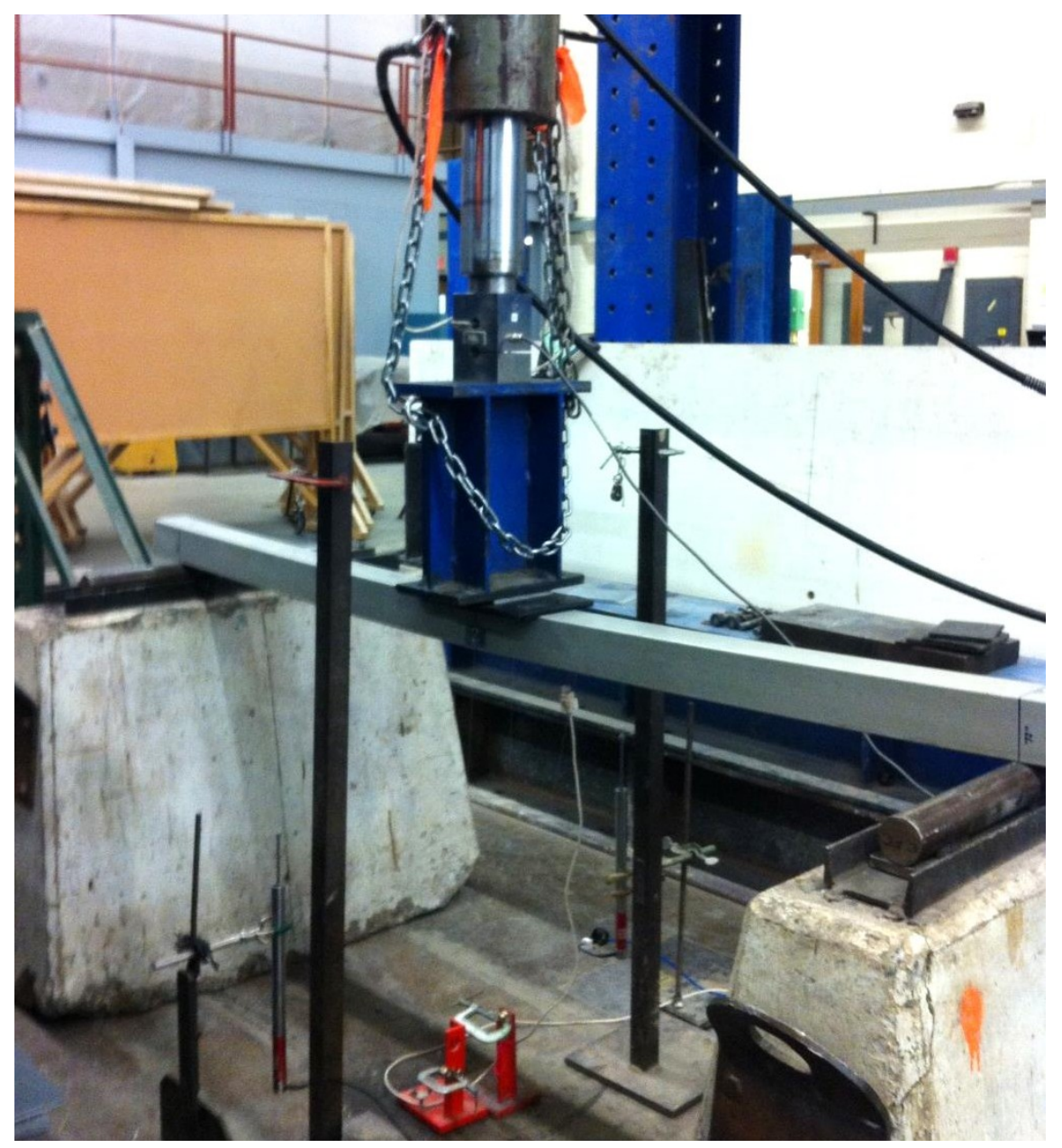

Figure 3-4 Setup using manually operated hydraulic actuator and steel load distributing spacer column for testing of a $3 " \times 3 " \times 0.25 "$ sample at a test span of 72 inches 


\subsection{Governing Modes of Failure}

The evaluation of differing cross-sectional dimensions of box sections at various spans revealed that the changes in failure modes corresponded with changes in span, depth, and web and flange thicknesses. Specifically, the failure mode was found to be most dependent on the span-to-depth $(L / h)$ ratio and the depth-to-thickness $(h / t)$ ratio. Observation of the physical nature of the failures and analysis of the experimental data led to an approximation of $L / h$ ratio thresholds for predicting the mode of failure. The three governing modes of failure controlling the flexural capacity of pultruded FRP box sections, relative to their approximate $L / h$ ratios, are: local buckling failure in the compression flange $(4 \leq L / h<6)$, combined strain failure at the webflange junction $(6 \leq L / h \leq 10)$, and bending failure in the tension flange $(10<L / h \leq 42)$. The beam response at $L / h$ ratios exceeding 42 is believed to be a function of geometric nonlinearity due to a relatively low longitudinal elastic modulus (approximately equal to $4.3 \mathrm{Msi}$ ), which is not further evaluated in this study.

\subsubsection{Local Buckling Failure in the Compression Flange}

For short-span beams $(4 \leq L / h<6)$, the failure mode is characterized as local instability caused by in-plane buckling in the compression flange. Local instability initiates when an individual component of a member fails, thus ultimately leading to global failure of the structural member. This is especially true in the case of thin-walled GFRP box beams. At very short spans, behavior is largely influenced by shear deformations because the shear modulus ( $G_{L T} \approx 0.42 \mathrm{Msi}$ ) is small in comparison to the longitudinal modulus ( $E_{L} \approx 4.3 \mathrm{Msi}$ ) (Ascione et al. 2012). Deformation in the compression flange under transverse loading leads to cracking at the web and compression flange junction, where the stress concentration is very high. Propagation of this localized cracking failure and the transference of the vertical load leads to crushing in the web 
between the top flange and the neutral axis. This phenomenon can be seen in Figure 3-5, where crushing of the web is clearly visible and the deformation of the elastomeric pads is an exaggeration of the deformed shape of the compression flange below it.

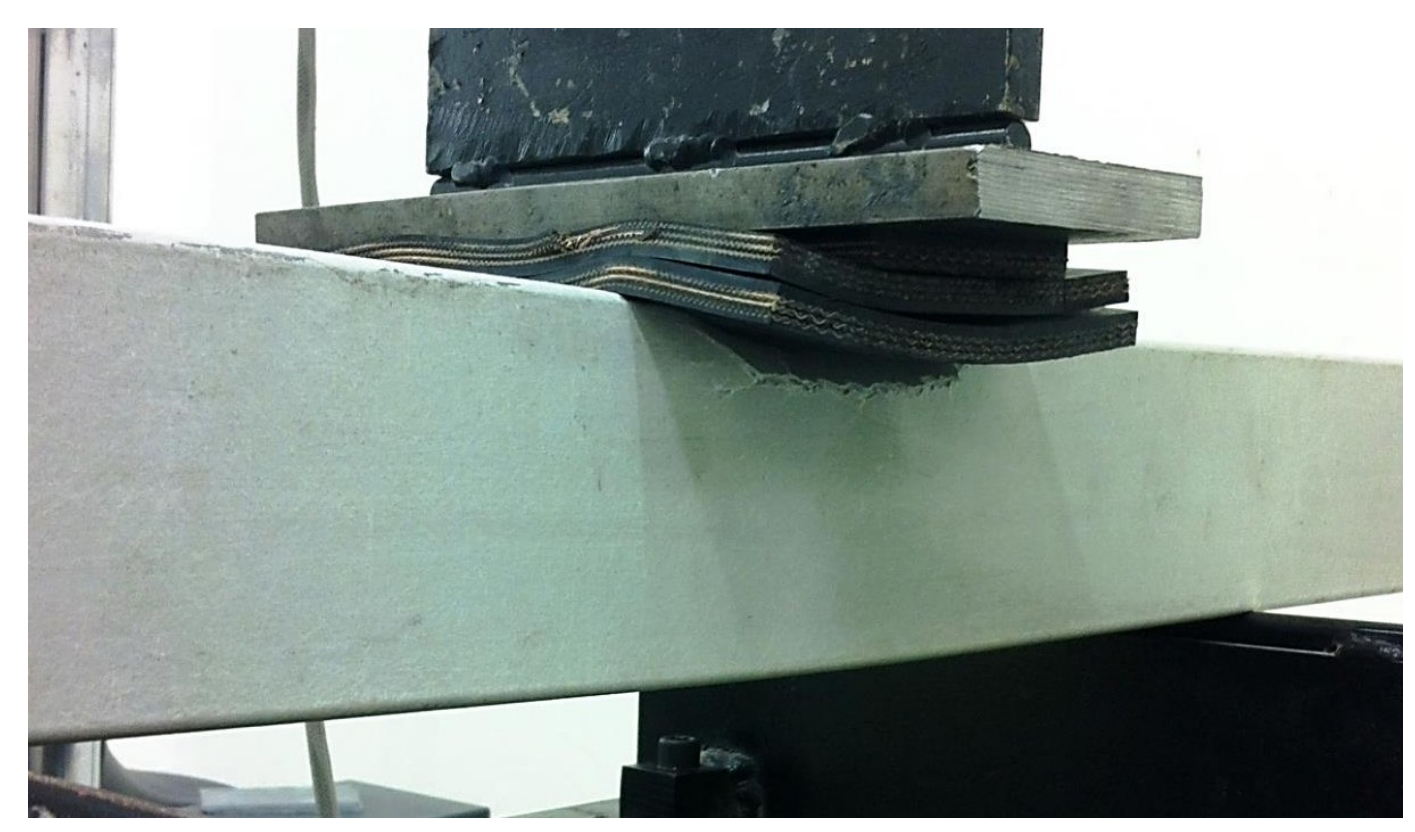

Figure 3-5 Web crushing caused by local instability in the compression flange

A variation of Equation (3-1), used to predict this mode of failure, was included in the proposed model of the most recently publicly available version of the ASCE/ACMA LRFD PreStandard (Nov. 2010) and was found by the author to be accurate at low $L / h$ ratios. Derived from the flange buckling equations for a rectangular box profile in axial compression by Bank (2006), and modified using Equation (3-9) where $M=P L / 4$ for a three-point bending scenario, the critical flexural load capacity, $P_{c r}$, can be calculated as shown in Equation (3-1)

$$
P_{c r}=\frac{\left(\frac{2 \pi^{2}}{3}\right)\left[\sqrt{0.88 E_{L, f} E_{T, f}}+v_{L T} E_{T, f}+2 G_{L T}\right] t_{f}{ }^{3} d}{L b_{f}} \times \frac{1}{(S I F)}
$$


In which

$E_{L, f}=$ characteristic value of the longitudinal modulus of the flange (psi)

$E_{T, f}=$ characteristic value of the transverse modulus of the flange (psi)

$v_{L T}=$ characteristic value of Poisson's ratio

$G_{L T}=$ characteristic value of the in-plane shear modulus (psi)

$t_{f}=$ compression flange thickness (in.)

$d=$ overall depth of section (in.) $=h-t_{f}$

$b_{f}=$ total flange width (in.)

$S I F=$ stress intensity factor, as computed using Equation (3-14)

In the case of local instability, the shear influence on deflection must also be considered given the low $L / h$ ratios. To keep calculations simple, a shear correction factor of $12 \%$ was used based on a study by Nagaraj and GangaRao (1997), and applied to the longitudinal modulus of the compression flange for all of the box beam prediction results within the span $4 \leq L / h<6$. This correction must be applied due to the large effect of shear on overall deflection at low $L / h$ ratios. The total deflection of a simply supported beam under three-point bending with load applied at the centerline can be expressed as shown in Equation (3-2)

$$
\delta_{\text {total }}=\delta_{\text {bending }}+\delta_{\text {shear }}=\frac{P L^{3}}{48 E I}+\frac{P L}{4 \alpha A G}
$$

In which

$\delta=$ deflection (in.)

$P=$ applied load (lbf)

$L=$ total span length (in.)

$E=$ modulus of elasticity (psi) 
$I=$ moment of inertia (in. ${ }^{4}$ )

$\alpha=$ shape factor

$A=$ cross-sectional area $\left(\right.$ in. $\left.^{2}\right)$

$G=$ shear modulus (psi)

Shape factors indirectly reveal the mechanical efficiency of material usage; that is, sections having equivalent cross-sectional area but differing in shape will not carry the same load in bending and shear. In general, shaped sections such as tubes, I-sections, or box beams are more efficient in resisting bending, torsional, and axial-compressive loads as compared to solid sections (Ashby 2005). The load condition and deformation region also has an effect; for example, the bending shape factor in the elastic region will not be the same as the bending shape factor at the onset of plasticity. This factor is dimensionless, dependent on the cross-sectional shape of the specimen, and accounts for the non-uniform shear stress distribution within the cross-section (Kassimali 2012). For box beams, the shape factor, $\alpha$, is considered to be in the range of $5 \%$ to $35 \%$ in the elastic bending region (Ashby 2005).

Structures are generally composed of beams and other structural elements having relatively large span-to-depth $(L / h)$ ratios to ensure that shear deformations are negligible in comparison to bending deformations. However, as span-to-depth ratio decreases, the magnitude of shear deformations in a member become considerable, and therefore must be considered in analysis. In order to simply illustrate the shear effect on deflection based on $L / h$ ratio, an example in which $b_{c}=0.8 b_{f}$ and $d_{w}=0.9 h$ is considered, where $b_{f}=$ total width of the flange, $b_{c}=$ clear spacing of the flange, $h=$ total height of the box section, and $d_{w}=$ clear depth of the web. Based on this example, a plot showing the percent of total deflection due to shear versus $L / h$ ratio can be seen in Figure 3-6. The curve in Figure 3-6 is generated by solving for $I / A$ in terms of $h$ and factoring 
out most of the material and shape constants in Equation (3-2). The ratio of $E / G$ is typically in the range of 5 to 7 for pultruded GFRP sections. For the purpose of this example, $E / G$ is assumed to be equal to 6. $E / G$ and $I / A$ can then be substituted back into Equation (3-2) to isolate the individual effects of bending and shear on total deflection, independent of material constants. The shear deflection is then found to be a function of the ratio $L / h$, indicating that as $L / h$ increases, the shear influence on deflection will decrease. From this plot, it can be seen clearly that at low $L / h$ ratios, the influence of shear on total deflection is significant; conversely, at large $L / h$ ratios, the influence of shear on total deflection is negligible. This critical stress prediction may not be accurate for highly anisotropic materials, where $E_{L} / E_{T}>5$ (Pre-Standard 2010).

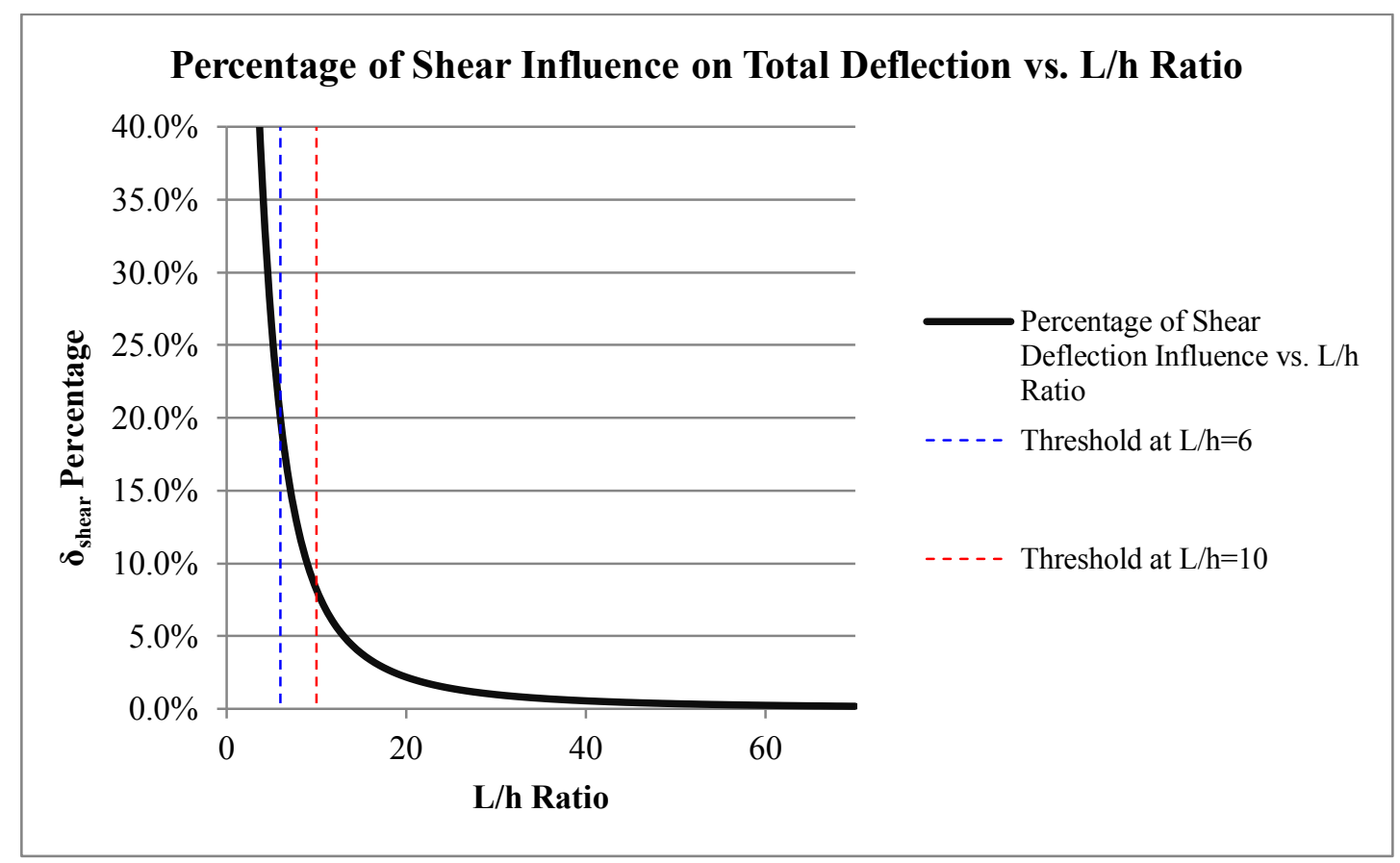

Figure 3-6 Percentage of shear influence on total deflection for specimen having $b_{c}=0.8 b_{f}$ and $d_{w}=0.9 h$

The application of the stress intensity factor, calculated using Equation (3-14) is crucial to the accuracy of this equation, as can be seen in Figure 3-7, which shows the difference in results before and after applying the SIF. 


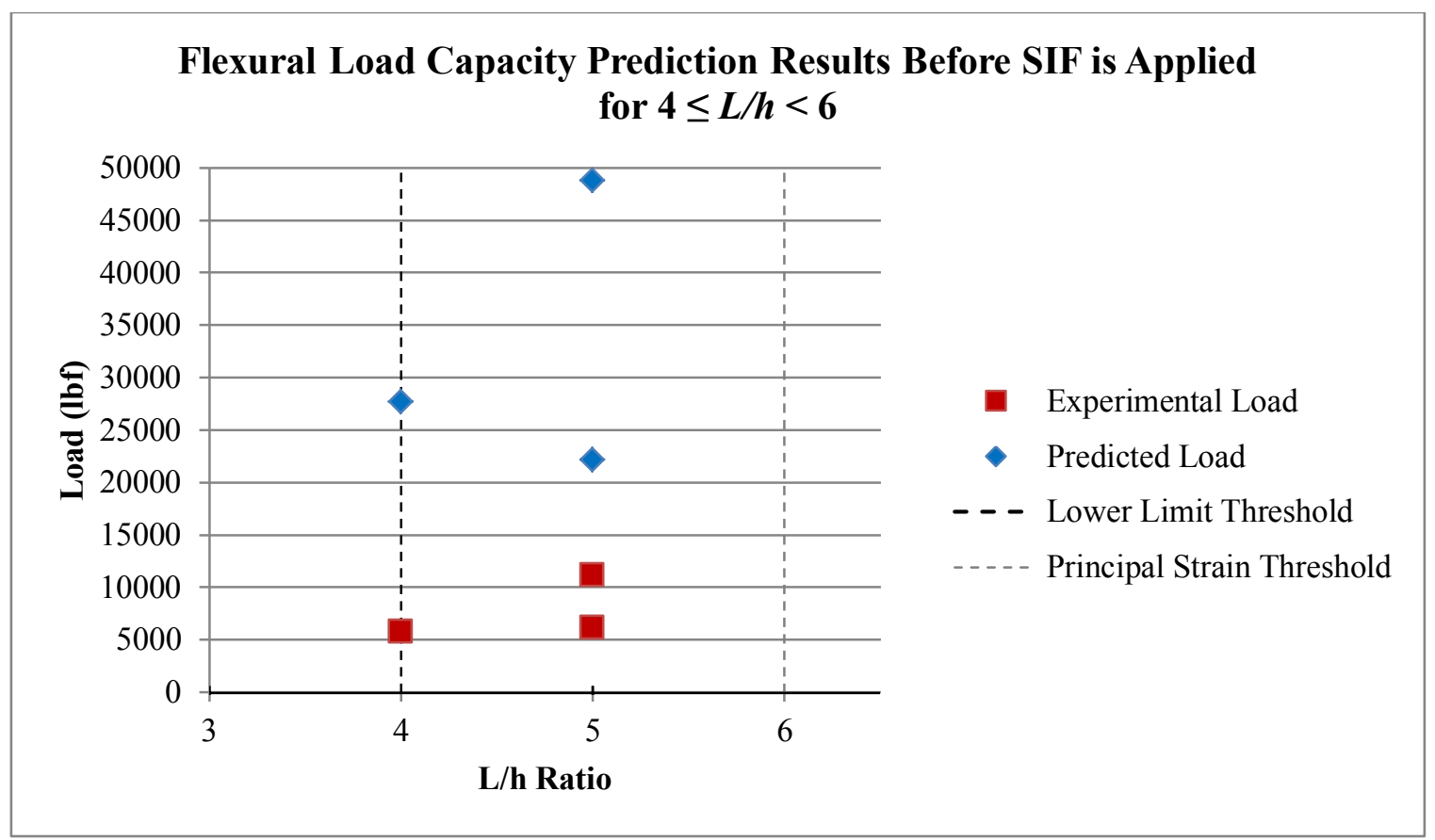

(a)

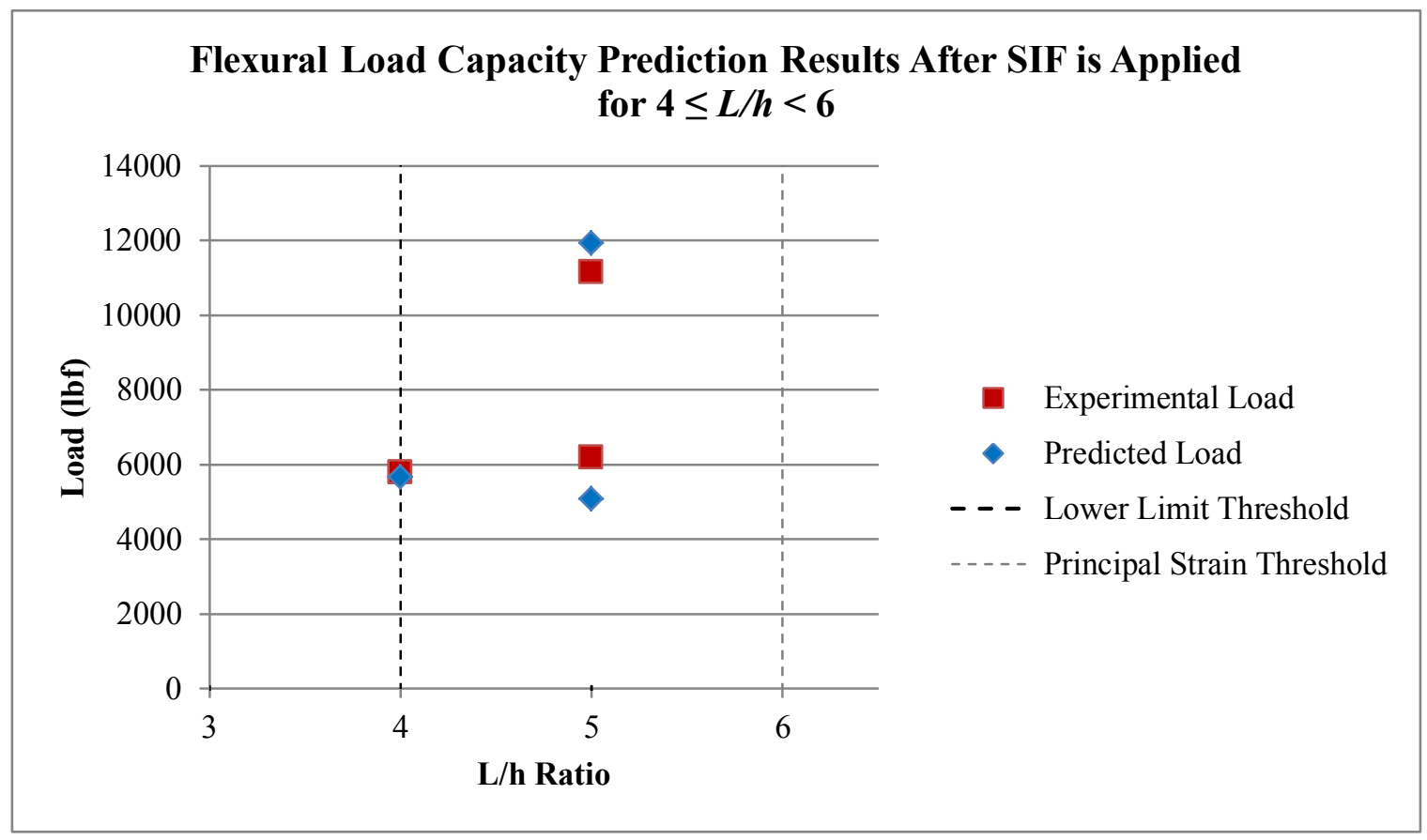

(b)

Figure 3-7 Comparison of predicted flexural load capacity versus experimental data for local buckling in the compression flange failure mode (a) before SIF is applied and (b) after SIF is applied 
By using Equation (3-1), prediction of the critical flexural capacity fell within $\pm 18.0 \%$ of experimental data for all points within the range $4 \leq L / h<6$. Prior to applying the stress intensity factor to this equation, the predicted flexural load capacity has a maximum error of $+379.4 \%$ when compared with experimental data for pultruded GFRP box sections included in this study.

\subsubsection{Principal Strain Failure in Tension Zone of the Web}

For intermediate span-to-depth beams $(6 \leq L / h \leq 10)$, the failure mode is characterized by a cracking failure at the reentrant corners of the tensile flange near midspan. Consequently, the near maximum longitudinal strains, transverse strains, and shear strains are induced at the reentrant corners of the flange, which results in higher magnitude strain-to-failure under combined effects (principal strain to failure). The combined strain is further amplified by the effect of stress concentration caused by the steep change in strain values at or near the webflange junction. Shear cracking begins to develop in the web near the tensile flange, and further propagates along the orientation angle of the principal compression strain through the tension zone of the web, leading to rupture due to opening of the crack caused by longitudinal tensile strain. Although the author observed only this type of tension-induced rupture during testing, the possibility of buckling due to localized compression strains exists in members having thinner walls, such as those found in carbon FRP box specimens. This type of failure mode is driven by the quantum jump in the shear strain at the web-flange junction.

In isotropic materials, the orientation of principal stresses and principal strains coincide at an angle of 45 degrees; this is not true for orthotropic materials, including pultruded GFRP beams, where principal stresses and strains do not necessarily coincide with the reinforcement axis (Hart-Smith 1989). Also, in comparison to steel, shear deformation is very high at low $L / h$ ratios due to the low shear modulus characteristic of an FRP, which allows for excessive angular 
deformation in the beam, known as shearing strain (Timoshenko 1962). Shear strain poses a major problem in pultruded GFRP beams where, since the main contributor to stiffness is the longitudinal fibers, the longitudinal elastic modulus is often about eight to ten times larger than the shear modulus. Unlike in isotropic materials, this imbalance in directional strength properties leads to disproportionate angular distortion (Popov 1954). By this reasoning, the maximum combined strain does not occur at the neutral axis in anisotropic box beams, but rather lies somewhere between the neutral axis and the tensile face of the beam.

Experimental evaluation by the author suggests that the orientation angle, $\theta$, for principal compression strain in pultruded GFRP composite beams lies somewhere between 35 and 40 degrees with respect to the longitudinal (pultruded) fiber axis. Critical shear cracking forms just above the web-flange junction, propagating perpendicular to the principal tensile strain, or in the direction of the aforementioned principal compression strain. This development of cracking was visible on a number of samples tested with $6 \leq \mathrm{L} / \mathrm{h} \leq 10$, such as the one shown in Figure 3-8.

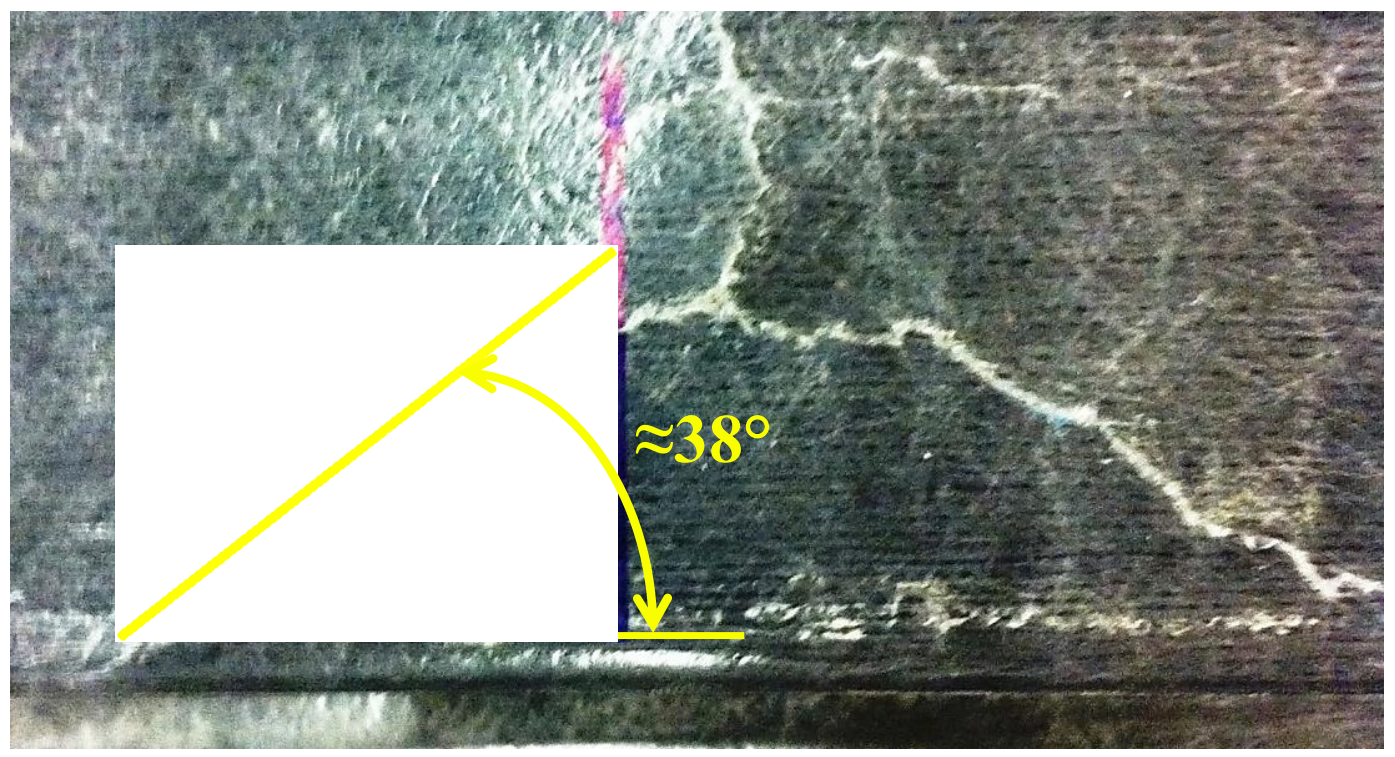

Figure 3-8 Crack formation visible in the tension zone of the web due to principal strain failure 
The mechanics of a combined strain is best explained using Mohr's circle for strain, which can be used to express the total strain at any point within a continuum. The Mohr's circle for strain can be drawn from the longitudinal strain, $\varepsilon_{x x}$, the transverse strain, $\varepsilon_{y y}$ (equal to longitudinal strain multiplied by Poisson's ratio), and the shear strain, $\gamma_{x y}$ (Beer et al. 2002). The center of the Mohr's circle is equal to the average normal strain, $\varepsilon_{\text {avg }}$, and can be expressed as shown in Equation (3-3)

$$
\varepsilon_{a v g}=\frac{\varepsilon_{x x}+\varepsilon_{y y}}{2}
$$

where the $\varepsilon_{\mathrm{xx}}$ and $\varepsilon_{\mathrm{yy}}$ strains are positive in tension and negative in compression. For most of the data collected, $\varepsilon_{\mathrm{yy}}$ was very close to $-\varepsilon_{\mathrm{xx}}$ multiplied by the Poisson's ratio, $\nu$. This relationship observation is based on the strain data collected by Constructed Facilities Center (CFC) researchers on numerous test specimens, and reported by Qureshi (2012).

The radius of Mohr's circle, $R$, is equal to one-half of the maximum shear strain, $\gamma_{\max }$, and can be expressed as shown in Equation (3-4) (Beer et al. 2002).

$$
R=\frac{\gamma_{\max }}{2}=\sqrt{\left(\frac{\varepsilon_{x x}-\varepsilon_{y y}}{2}\right)^{2}+\left(\frac{\gamma_{x y}}{2}\right)^{2}}
$$

The theoretical maximum shearing strains calculated from experimental load data and coupon-based material properties ranged from $16,400 \mu \varepsilon$ to $19,500 \mu \varepsilon$ after applying a stress intensity factor (SIF), discussed in depth later. These theoretical web shear strain values match well with the range recorded by the author during testing. An example of principal strain and maximum shear strain calculations is shown in Appendix A.

Based on Equation (3-4), an equation was developed to directly relate the ultimate shearing strain to the failure load. This was accomplished by substituting basic mechanics of materials 
strain relations, shown in Equations (3-5) through (3-7), into Equation (3-4), and applying a stress intensity factor (SIF) which is further explained in Section 3.4.

$$
\begin{gathered}
\varepsilon_{x x}=\frac{P L c}{4 E_{L} I} \\
\varepsilon_{y y}=-v \varepsilon_{x x} \\
\gamma_{x y}=\frac{1.5 P}{2 t_{w} h G_{L T}} \times \frac{1}{2}
\end{gathered}
$$

In which

$\varepsilon_{X X}=$ transverse strain

$\varepsilon_{y y}=$ longitudinal strain

$\gamma_{x y}=$ shear strain

$P=$ applied load (lbf)

$L=$ total span length (in.)

$c=$ distance to the neutral axis from inside of flange (in.) $=d_{w} / 2$ for symmetric box sections

$E_{L}=$ characteristic value of the longitudinal modulus (psi)

$v_{L T}=$ characteristic value of Poisson's ratio

$G_{L T}=$ characteristic value of the in-plane shear modulus (psi)

$t_{w}=$ thickness of one web (in.)

$h=$ total height of section (in.)

It should be noted here that the factor of one-half is applied to Equation (3-7) to account for the presence of two webs in symmetrical box sections. By making these substitutions, the variables can be rearranged to isolate the predicted critical flexural load capacity, $P_{c r}$, as shown in Equation (3-8) 


$$
P_{c r}=\left[\frac{4 \gamma_{\max } G_{L T} h t_{w}}{(S I F)}\right] \times \frac{1}{\sqrt{2.25+\left[\frac{\left(1+v_{L T}\right) L c G_{L T} h t_{w}}{E_{L, w} I_{X}}\right]^{2}}}
$$

In which

$P_{c r}=$ predicted critical flexural load capacity (lbf)

$\gamma_{\max }=$ mean value of maximum shear strain at failure based on experimental data

$G_{L T}=$ characteristic value of the in-plane shear modulus (psi)

$h=$ total height of the section (in.)

$t_{w}=$ thickness of the web (in.)

SIF $=$ stress intensity factor, as computed using Equation (3-14)

$v_{L T}=$ characteristic value of Poisson's ratio

$L=$ total span length (in.)

$c=$ distance from the neutral axis to inner wall of the flange (in.)

$E_{L, w}=$ characteristic value of the longitudinal modulus of the web (psi)

$I_{x}=$ strong-axis moment of inertia of the section (in. ${ }^{4}$ )

By simplifying the equation based on relevant numerical assumptions, it can be shown that the load at failure is largely dependent on the ratio of span length, $L$, multiplied by the wall thickness, $t_{w}$, and divided by the area of the flange, $A_{f}$, written as $L t / A_{f}$. Application of the stress intensity factor yields consistently accurate results, as shown in Figure 3-9. In design, strength properties and the typical shearing strain to failure of the specimen will be provided by the manufacturer. This cataloged data becomes necessary because the strength properties are largely dependent on the fiber architecture of the specimens, which varies widely between manufacturers. 


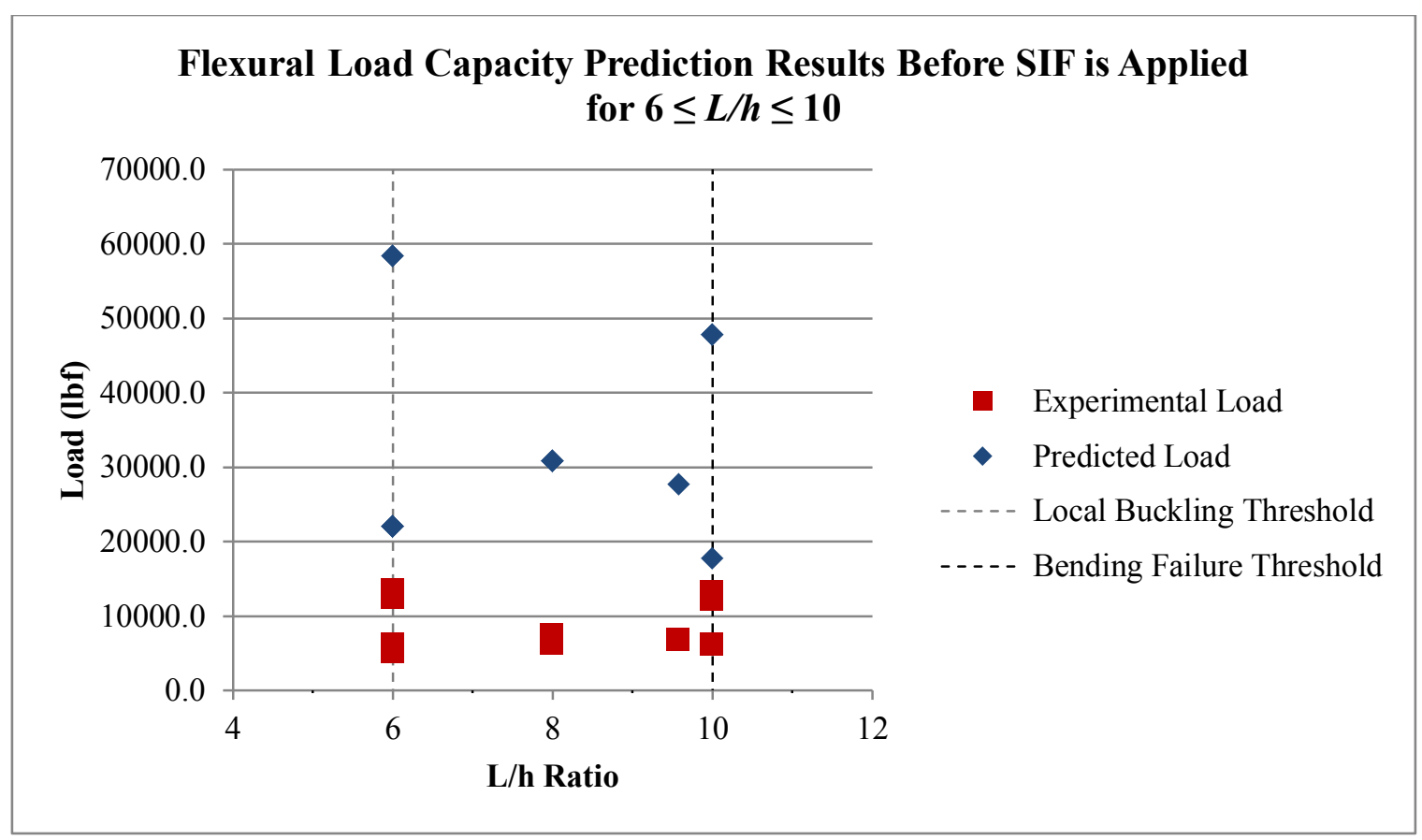

(a)

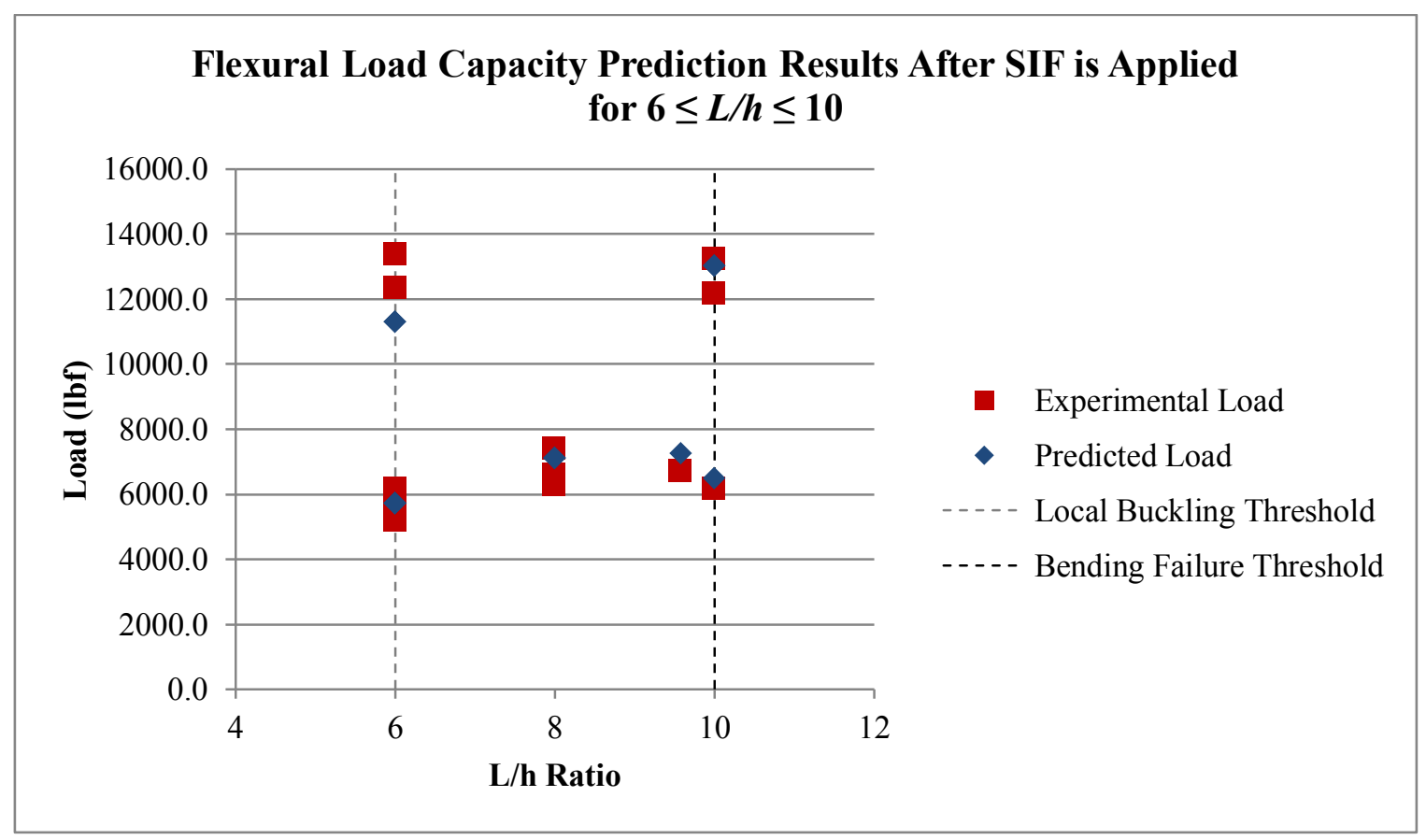

(b)

Figure 3-9 Comparison of predicted flexural load capacity versus experimental data for principal strain failure in the tension zone of the web (a) before SIF is applied and (b) after SIF is applied 
By substituting a mean value for $\gamma_{\max }$ based on experimental data, and dividing by the stress intensity factor as calculated in Equation (3-14), prediction of the critical flexural load capacity fell within $\pm 15.5 \%$ for all specimens tested within the $L / h$ range of 6 to 10 . Prior to applying the SIF, prediction of the critical flexural load capacity is highly inaccurate, yielding a maximum error of $+392.1 \%$.

\subsubsection{Bending Failure in the Tension Flange}

Beams tested at long spans $(10<L / h \leq 42)$ exhibited classic bending failure in the tension flange, as shown in Figure 3-11. From stress-strain data, it is known that GFRP box beams display mostly elastic behavior leading up to failure. When stresses remain in the elastic range, the neutral axis coincides with the centroid of the cross-section (Beer et al. 2002). Therefore, accurate prediction of the critical flexural load capacity can be achieved using the classic pure bending formula with coupon-based material properties and applying a stress intensity factor (SIF). The classic bending stress formula is as shown in Equation (3-9)

$$
\sigma=\frac{M c}{I}
$$

In which

$\sigma=$ flexural stress (psi)

$M=$ moment about the neutral axis $=P L / 4$ for three-point bending $(\mathrm{lbf} \cdot \mathrm{in}$.

$c=$ distance to the neutral axis $=h / 2$ for symmetric box sections for extreme fibers (in.)

$I=$ moment of inertia about the neutral axis $=\frac{1}{12}\left(b_{f} h^{3}-b_{c} d_{w}^{3}\right)$ for symmetric box sections (in. $\left.{ }^{4}\right)$

$L=$ total span length (in.)

$h=$ total depth of section (in.)

$b_{f}=$ full width of flange (in.) 
$b_{c}=$ clear spacing of flange (in.)

$d_{w}=$ depth of web $=h-2 t$ (in.)

By substituting in the relations shown above, and allowing the flexural stress to be taken as the longitudinal strength in the flange, $F_{L f}$, the predicted critical flexural load capacity, $P_{c r}$, can be calculated as shown in Equation (3-10). The longitudinal strength in the flange shall be obtained from tension testing of coupon samples, as described in Appendix C.

$$
P_{c r}=\frac{8 F_{L f} I_{x}}{L h} \times \operatorname{SIF}
$$

In which

$P_{c r}=$ predicted critical flexural load capacity $(\mathrm{lbf})$

$F_{L f}=$ characteristic longitudinal strength in the flange acquired from tension coupon testing (psi)

$I_{x}=$ strong-axis moment of inertia of the section (in. ${ }^{4}$ )

$L=$ total span length (in.)

$h=$ total height of the section (in.)

$S I F=$ stress intensity factor, as computed using Equation (3-14)

Prior to application of the stress intensity factor (SIF), prediction using this equation is highly inaccurate, especially as $L / h$ ratio approaches the proposed threshold at $L / h=10$. Once the SIF has been applied, prediction of the critical flexural load capacity is vastly improved. This phenomenon is clearly visible in Figure 3-10, which shows a comparison of the accuracy of the prediction prior to, and after, the stress intensity factor has been applied. As can be seen in the figure, the predicted flexural load capacity agrees well with experimental load data included in this study once the SIF is applied, with over $71 \%$ of all predicted values within $\pm 10 \%$ error from the experimental value. Before applying the SIF, the maximum experimental error is $+226.5 \%$. 


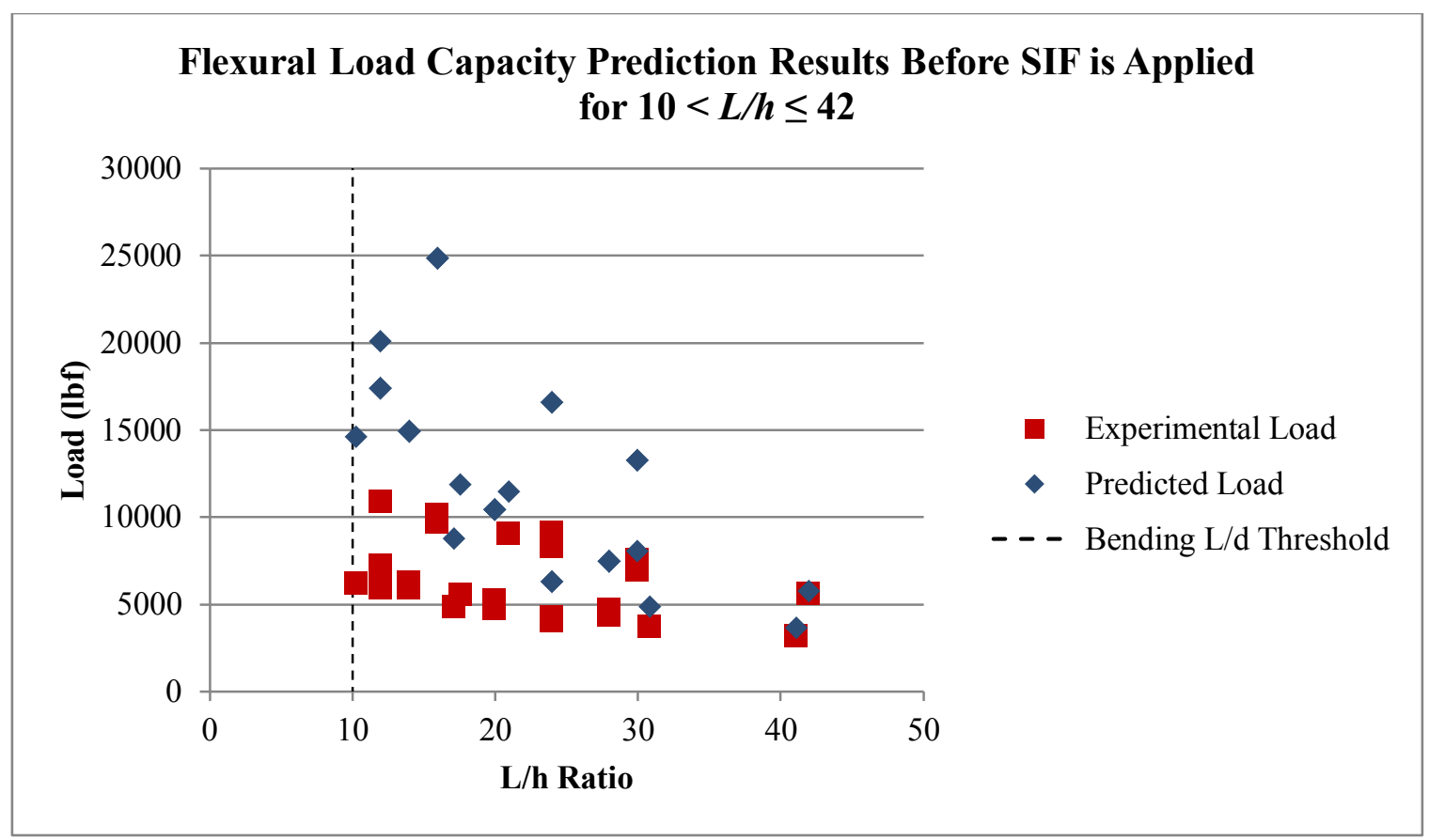

(a)

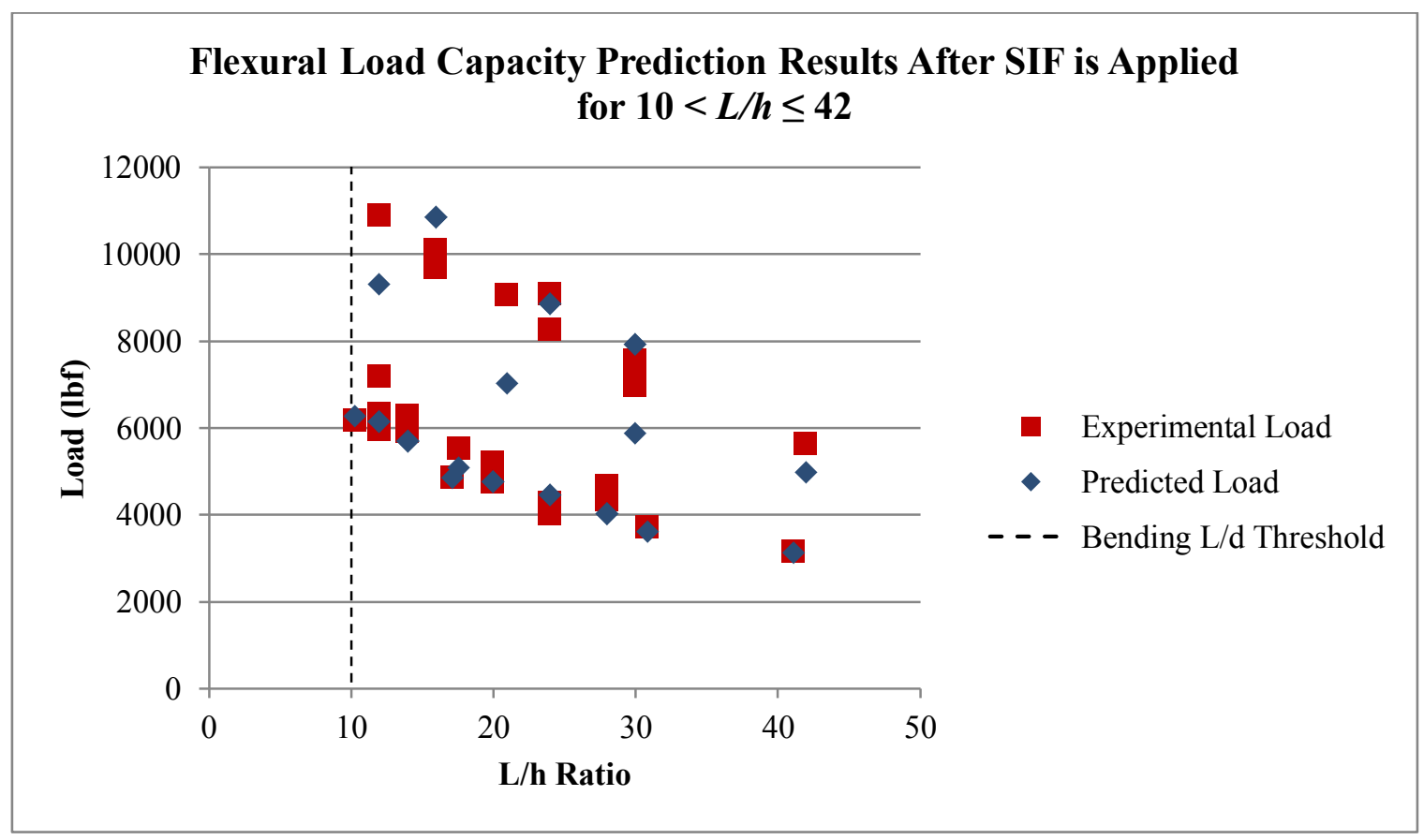

(b)

Figure 3-10 Comparison of predicted flexural load capacity versus experimental data for bending failure in the tension flange (a) before SIF is applied and (b) after SIF is applied 


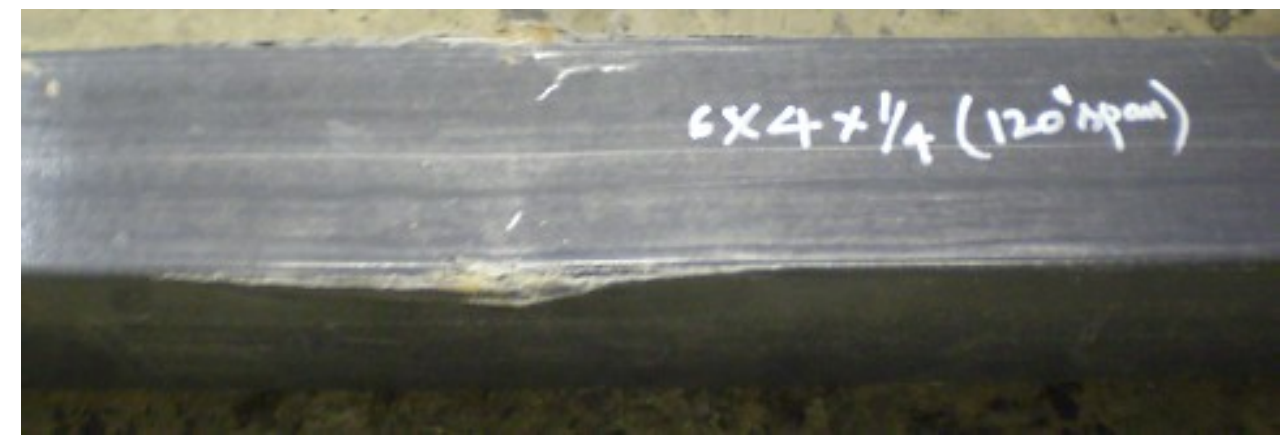

Figure 3-11 Classic bending failure in the tension flange of a $6 " \times 4 " \times 0.25 "$ box specimen (Qureshi 2012)

\subsection{Stress Intensity Factor}

Stress intensity factors are particularly important in materials whose stress-strain relationship at failure is revealed to be brittle. Most brittle materials possess a fracture strength lower than calculated theoretical values based on atomic bonding energies, caused by microscopic interior and surface defects which exist under normal conditions. Stresses become amplified both at the location of such flaws and at macroscopic discontinuities such as sharp corners or notches. As the stress at one of these cracks or flaws exceeds the critical stress value, the crack will extend or propagate until fracture occurs (Callister 2007). A stress intensity factor is used to predict the stress state at a defect location caused by the loading conditions. Because FRP composites exhibit mostly elastic behavior prior to failure, local stress intensity effects become a crucial component of design (Timoshenko 1966). This component is further complicated by the bi-axial or tri-axial stress conditions characteristic of FRP composites under increasing strains (ASCE 1984).

Sudden changes in cross-section, such as reentrant corners in tubular members, cause the formation of irregularities in stress distribution. These irregularities translate to stresses exceeding the average value, which often leads to progressive crack formation at these locations 
(Timoshenko 1966). For rolled steel profile sections, Trefftz (1922) stated that the maximum stress at reentrant corners in members subjected to torsion could be predicted by multiplying the calculated stress by an amplification factor, $k$, given by Equation (3-11)

$$
k=1.74 \times \sqrt[3]{\frac{t}{r}}
$$

In which

$t=$ thickness of the flange (in.)

$r=$ radius of the fillet (in.)

Through empirical analysis, it was found that this same equation, omitting the factor of 1.74 which applies only to metals, could be modified and applied to pultruded GFRP box sections subjected to transverse loading. Thus, the equation can then be written as shown in Equation (3-12)

$$
k \propto \sqrt[3]{\frac{t}{r_{i}}}
$$

In which

$t=$ wall thickness (in.)

$r_{i}=$ inner radius of the reentrant corner (in.)

It should be noted that $k$ increases as $r_{i}$ decreases, implying that sharper corners result in higher stress concentrations (Timoshenko 1966). The stress intensity factor, $k_{i}$ is also considered to be a function of both the $L / h$ ratio and $h / t$ ratio, which can be combined into a single empirical stress intensity factor calculated as shown in Equation (3-13) 


$$
k_{i}=\sqrt{\frac{h / t}{L / h}}=\frac{h}{\sqrt{L t}}
$$

In which

$h=$ total height of section (in.)

$L=$ total span length (in.)

$t=$ wall thickness (in.)

This component of the stress intensity factor results from understanding that strain can be written as a function of the $L / h$ ratio and the shape factor for failure under bending is a function of the $h / t$ ratio. A loose derivation of this factor is provided in Appendix B. A more rigorous study of this factor is required to confirm the relationship and derivation presented within. By multiplying the effect of the stress amplification at the reentrant corner by the combined effect of the $L / h$ and $h / t$ ratios, the result is one quantifiable, dimensionless stress intensity factor (SIF) that is dependent on known section properties and span length, as shown in Equation (3-14).

$$
S I F=k k_{i}=\frac{k h}{\sqrt{L t}}
$$

In which

$k=$ corner stress amplification factor, as calculated using Equation (3-12)

$h=$ total height of section (in.)

$L=$ total span length (in.)

$t=$ wall thickness (in.)

Inclusion of all significant dimensions of the cross-section of the specimen makes the stress intensity factor applicable to multiple modes of failure. Initial development of the stress 
intensity factor was based on empirical evaluation of data, and has been linked to the principal strain due to flexure as shown in Appendix B. Further evaluation of the stress intensity factor is necessary to fully describe the mechanics behind its application.

\subsection{Existing Critical Flexural Capacity Prediction Models}

For comparison purposes, two models from previous draft versions of the ASCE/ACMA Pre-Standard for Load and Resistance Factor Design (LRFD) of Pultruded Fiber Reinforced Polymer (FRP) Structures were applied and equated to the experimental data obtained for this study. The first model comes from the most recently publicly available draft of the ASCE/ACMA LRFD Pre-Standard proposed in November 2010. It should be noted that for

direct comparison to the model proposed in Section 3.3, no resistance factors will be applied. For square and rectangular box members, two equations must be applied, and the minimum critical load value from these two equations is taken as the critical flexural load capacity. The first equation is used to calculate the nominal strength of member due to material rupture in tension or compression in the flanges or webs of members subjected to flexure, as calculated using Equation (3-15)

$$
M_{n}=\min \left\{\frac{F_{L, f}\left(E_{L, f} I_{f}+E_{L, w} I_{w}\right)}{y_{f} E_{L, f}}, \frac{F_{L, w}\left(E_{L, f} I_{f}+E_{L, w} I_{w}\right)}{y_{w} E_{L, w}}\right\}
$$

In which

$F_{L, f}=$ characteristic longitudinal strength of the flange (psi)

$F_{L, w}=$ characteristic longitudinal strength of the web (psi)

$E_{L, f}=$ characteristic value of the longitudinal modulus of the flange (psi)

$E_{L, w}=$ characteristic value of the longitudinal modulus of the web (psi) 
$I_{f}=$ moment of inertia of the flanges about the axis of bending (in. $\left.{ }^{4}\right)$

$I_{w}=$ moment of inertia of the webs about the axis of bending (in. $\left.{ }^{4}\right)$

$y_{f}=$ distance from the neutral axis to the extreme fiber of the flange (in.)

$y_{w}=$ distance from the neutral axis to the extreme fiber of the web (in.)

It is noted that when members have a longitudinal elastic modulus in the flange that is within 15 percent of the longitudinal elastic modulus of the web, the equation can be simplified as shown in Equation (3-16). For this study, it is assumed that the longitudinal elastic moduli in the flange and web are equal, therefore, the equation becomes

$$
M_{n}=\frac{F_{L} I}{y}
$$

In which

$F_{L}=$ characteristic longitudinal strength of the member (psi)

$I=$ moment of inertia of the member about the axis of bending (in. ${ }^{4}$ )

$y=$ distance from the neutral axis to the extreme fiber of the member (in.)

The second equation required by this draft version is for the nominal strength of members due to local instability. All members that undergo compressive stresses due to flexure must be checked for local buckling of the flanges and webs. The local instability of the flange or web of a square or rectangular box section shall be determined by either Equation (3-17), for compression flange local buckling, or Equation (3-18), for web local buckling.

$$
\begin{aligned}
& M_{n}=f_{c r} \frac{E_{L, f} I_{f}+E_{L, w} I_{w}}{y E_{L, f}} \\
& M_{n}=f_{c r} \frac{E_{L, f} I_{f}+E_{L, w} I_{w}}{y_{f} E_{L, w}}
\end{aligned}
$$


In which

$f_{c r}=$ critical buckling stress taken as the minimum of compression flange local buckling from Equation (3-20), and web local buckling from Equation (3-23)

$E_{L, f}=$ characteristic value of the longitudinal modulus of the flange (psi)

$E_{L, w}=$ characteristic value of the longitudinal modulus of the web (psi)

$I_{f}=$ moment of inertia of the flanges about the axis of bending (in. $\left.{ }^{4}\right)$

$I_{w}=$ moment of inertia of the webs about the axis of bending (in. ${ }^{4}$ )

It is noted that when members have a longitudinal elastic modulus in the flange that is within 15 percent of the longitudinal elastic modulus of the web, the Equations (3-17) and (3-18) can be simplified as shown in Equation (3-19). For this study, it is assumed that the longitudinal elastic moduli in the flange and web are equal, therefore, the equation becomes

$$
M_{n}=\frac{f_{c r} I}{y}
$$

In which

$f_{c r}=$ critical buckling stress taken as the minimum of compression flange local buckling from Equation (3-20), and web local buckling from Equation (3-23)

$I=$ moment of inertia of the member about the axis of bending (in. $\left.{ }^{4}\right)$

$y=$ distance from the neutral axis to the extreme fiber of the member (in.)

The critical buckling stress, $f_{c r}$, due to compression flange local buckling is given by Equation (3-20)

$$
f_{c r}=\frac{4 \pi^{2} t_{f}^{2}}{b_{f}^{2}}\left[\frac{\sqrt{\left(E_{L, f} E_{T, f}\right)(1+4.1 \xi)}}{6}+\left(2+0.62 \xi^{2}\right)\left(\frac{E_{T, f} v_{L T}}{12}+\frac{G_{L T}}{6}\right)\right]
$$


Where

$$
\begin{gathered}
\xi=\frac{1}{1+\frac{4 E_{T, f} t_{f}^{3}}{5 k_{r} b_{f}}} \\
k_{r}=\frac{E_{T, w} t_{w}^{3}}{3 h}\left\{1-\left[\left(\frac{2 t_{f}^{2} h^{2} E_{L, f}}{11.1 b_{f}^{2} t_{w}^{2} E_{L, f}}\right)\left(\frac{\sqrt{E_{L, f} E_{T, f}}+E_{T, f} v_{L T}+2 G_{L T}}{1.25 \sqrt{E_{L, w} E_{T, w}}+E_{T, w} v_{L T}+2 G_{L T}}\right)\right]\right\}
\end{gathered}
$$

The critical buckling stress, $f_{c r}$, due to web local buckling is given by Equation (3-23)

$$
f_{c r}=\frac{11.1 \pi^{2} t_{w}^{2}}{6 h^{2}}\left(1.25 \sqrt{E_{L, w} E_{T, w}}+E_{T, w} v_{L T}+2 G_{L T}\right)
$$

In which

$E_{L, f}=$ characteristic value of the longitudinal modulus of the flange (psi)

$E_{L, w}=$ characteristic value of the longitudinal modulus of the web (psi)

$E_{T, f}=$ characteristic value of the transverse modulus of the flange (psi)

$E_{T, w}=$ characteristic value of the transverse modulus of the web (psi)

$G_{L T}=$ characteristic value of the in-plane shear modulus (psi)

$v_{L T}=$ characteristic longitudinal Poisson's ratio (in absence of available data $v_{L T}=0.3$ )

$b_{f}=$ full width of the flange (in.)

$h=$ full height of the member (in.)

$t_{f}=$ thickness of the flange (in.)

$t_{w}=$ thickness of the web (in.)

$\xi=$ coefficient of restraint

$k_{r}=$ rotational spring constant $(\mathrm{lbf} / \mathrm{rad})$

The second model comes from a more recent draft of the ASCE/ACMA LRFD PreStandard proposed in May 2013. It should be noted that for direct comparison to the model 
proposed in Section 3.3, no resistance factors will be applied. In this newer draft, only one equation must be applied to determine the critical flexural load capacity of a square or rectangular box member. This single equation is based on the nominal strength of members due to local buckling, calculated as shown in Equation (3-24)

$$
M_{n}=S_{t} F_{c r}
$$

In which

$S_{t}=$ transformed section modulus about the axis of bending (in. ${ }^{3}$ ), taking into account conditions of force equilibrium and strain compatibility $F_{c r}=$ critical buckling stress, taken as the lower value of local buckling stress of the compression flange from Equation (3-25) and local buckling stress of the web from Equation (3-26)

The local buckling stress of the compression flange, $F_{c r f}$, shall be calculated as shown in Equation (3-25), while the local buckling stress of the web, $F_{c r w}$, is defined as shown in Equation

$$
\begin{gathered}
F_{c r f}=\frac{\left(\frac{\pi^{2}}{6}\right)\left[\sqrt{E_{L, f} E_{T, f}}+E_{T, f} v_{L T}+2 G_{L T}\right]}{\left(\frac{b_{f}}{t_{f}}\right)^{2}} \\
F_{c r w}=\frac{15 \sqrt{E_{L, w} E_{T, w}}}{\left(\frac{d}{t_{w}}\right)^{2}}
\end{gathered}
$$

In which

$E_{L, f}=$ characteristic value of the longitudinal modulus of the flange (psi)

$E_{L, w}=$ characteristic value of the longitudinal modulus of the web (psi)

$E_{T, f}=$ characteristic value of the transverse modulus of the flange (psi)

$E_{T, w}=$ characteristic value of the transverse modulus of the web (psi) 
$G_{L T}=$ characteristic value of the in-plane shear modulus (psi)

$v_{L T}=$ characteristic longitudinal Poisson's ratio (in absence of available data $v_{L T}=0.3$ )

$b_{f}=$ full width of the flange (in.)

$t_{f}=$ thickness of the flange (in.)

$d=$ overall depth of section (in.) $=h-t_{f}$

$t_{w}=$ thickness of the web (in.)

Results of the critical flexural capacity prediction models from the aforementioned drafts of the ASCE/ACMA LRFD Pre-Standard, compared with the experimental load capacities obtained for this study, can be found in Section 3.7.

\subsection{Results and Discussion}

Under the proposed model, the failure mode and flexural load at failure can be accurately predicted for pultruded GFRP box sections possessing span-to-depth $(L / h)$ ratios ranging from 4 to approximately less than 42. Figure 3-12 demonstrates the accuracy of the prediction model for each of the three governing modes of failure discussed in Sections 3.3.1 through 3.3.3. As seen in the plot below, nearly all of the data lies within $\pm 15 \%$ experimental error. 


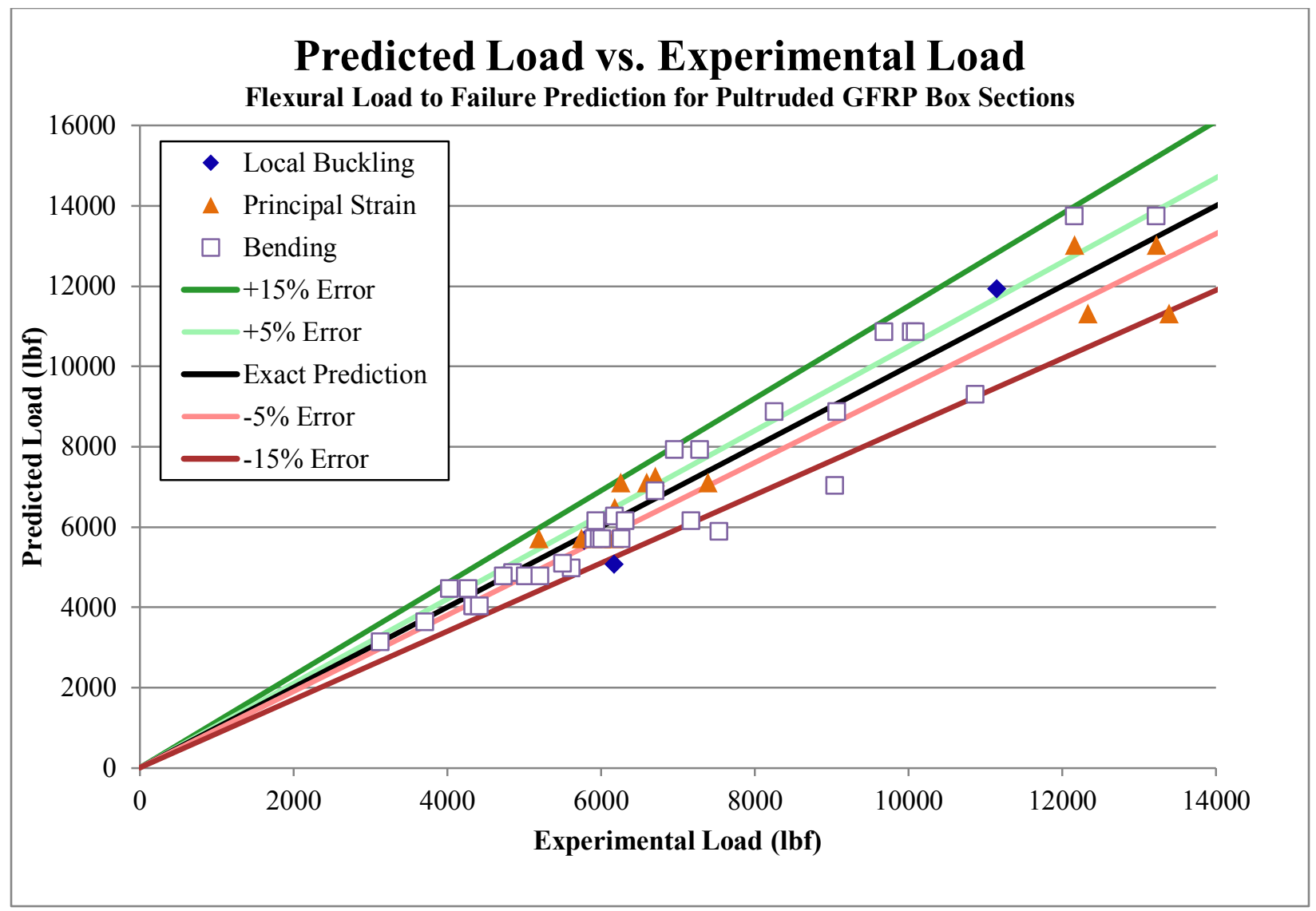

Figure 3-12 Predicted load vs. experimental load based on proposed flexural load capacity prediction model

\subsubsection{Results for $4 \leq L / h<6$}

At very short spans, a local instability leading to local buckling in the compression flange controls the failure of the member. Using Equation (3-1), which is a modified version of the equation found in the ASCE/ACMA LRFD Pre-Standard (Pre-Standard 2010), flexural load capacity prediction fell within $\pm 18.0 \%$ of experimental data for sections tested within this range of $L / h$ ratios, as shown in Table 3-3. The mean error for Equation (3-1) for the range $4 \leq L / h<6$ based on experimental data included in this study is $-9.7 \%$. 
Table 3-3 Flexural load capacity prediction results for box members where $4 \leq L / h<6$ using Equation (3-1)

\section{Equation (3-1)}

\begin{tabular}{|c|c|c|c|c|c|c|c|c|c|}
\hline Section & L (in.) & b $_{\text {f }}$ (in.) & h (in.) & $\mathbf{t}$ (in.) & L/h & $\begin{array}{c}\text { Number of } \\
\text { Replications }\end{array}$ & $\mathbf{P}_{\text {expt }}$ (lbf) & $\mathbf{P}_{\text {cr }}$ (lbf) & \% Error \\
\hline \hline \multirow{2}{6}{$" \times \mathbf{4} \times \mathbf{0 . 2 5 "}$} & 24 & 4 & 6 & 0.25 & 4 & 1 & 5783 & 5662.0 & $-2.1 \%$ \\
\cline { 2 - 10 } & 30 & 4 & 6 & 0.25 & 5 & 1 & 6178 & 5064.2 & $-18.0 \%$ \\
\hline 6"×6"×0.375" & 30 & 6 & 6 & 0.375 & 5 & 1 & 11153 & 11926.1 & $6.9 \%$ \\
\hline
\end{tabular}

Note: Experimental loads highlighted in yellow were tested by Qureshi (2012)

As can be seen in Table 3-3, overall the prediction based on the proposed model within this range of $L / \mathrm{h}$ ratios. The source of this error could be due to a variety of reasons, including variances in test setup and loading rate, as well as manufacturing inconsistencies. However, it should be noted that Equation (3-1) neglects resistance provided by the web, which could result in as much as a $25 \%$ reduction in prediction of the critical flexural load, $P_{c r}$. Another possible source of error stems from unknown (exact) boundary condition effects between the compression flange and the web leading to the plate buckling phenomenon. It is very likely that as the threshold at $L / h$ equal to four is approached, the boundary condition is changing, resulting in a more significant compression effect in the web, and less influence overall due to bending. Only a few replications were performed within this range of $L / h$ ratio; therefore, further testing would refine the accuracy of the local buckling in the compression flange prediction equation.

\subsubsection{Results for $6 \leq L / h \leq 10$}

For intermediate spans $(6 \leq L / h \leq 10)$, failure is governed by combined (axial and shear) strain failure at the web-flange junction. Using Equation (3-8), flexural load capacity prediction fell within $\pm 15.5 \%$ of experimental data for pultruded box sections within this range of $L / h$ ratios, as shown in Table 3-4. The results shown in Table 3-4 are based on generalized strength and stiffness properties based on the manufacturer. By inserting specific strength and stiffness 
properties provided for each section size based on coupon testing by the manufacturer, the flexural load capacity can be more accurately predicted. Some of the resulting error could also be attributed to variances in test setup and loading rate, as well as manufacturing inconsistencies. The mean error for Equation (3-8) for the range $6 \leq L / h \leq 10$ based on experimental data included in this study is $+0.7 \%$.

Table 3-4 Flexural load capacity prediction results for box members where $6 \leq L / h \leq 10$ using Equation (3-8)

\begin{tabular}{|c|c|c|c|c|c|c|c|c|c|}
\hline \multirow{2}{*}{ Section } & \multirow[b]{2}{*}{ L (in) } & \multirow[b]{2}{*}{$b_{\mathrm{f}}$ (in.) } & \multirow[b]{2}{*}{$h$ (in.) } & \multirow[b]{2}{*}{ t (in.) } & \multirow[b]{2}{*}{$\mathrm{L} / \mathrm{h}$} & \multirow[b]{2}{*}{$\begin{array}{l}\text { Number of } \\
\text { Replications }\end{array}$} & \multirow[b]{2}{*}{$\mathbf{P}_{\text {expt }}(\mathrm{lbf})$} & \multicolumn{2}{|c|}{ Equation (3-8) } \\
\hline & & & & & & & & $\mathbf{P}_{\mathrm{cr}}(\mathrm{lbf})$ & \% Error \\
\hline \multirow[t]{5}{*}{$3 " \times 3 " \times 0.25 "$} & 18 & 3 & 3 & 0.25 & 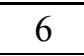 & 1 & 5194 & 5719.4 & $10.1 \%$ \\
\hline & 18 & 3 & 3 & 0.25 & 6 & 1 & 5749 & 5719.4 & $-0.5 \%$ \\
\hline & 18 & 3 & 3 & 0.25 & 6 & 1 & 6177 & 5719.4 & $-7.4 \%$ \\
\hline & 18 & 3 & 3 & 0.25 & 6 & 1 & 6015 & 5719.4 & $-4.9 \%$ \\
\hline & 30 & 3 & 3 & 0.25 & 10 & 1 & 6187 & 6478.7 & $4.7 \%$ \\
\hline \multirow[t]{4}{*}{$6 " \times 4 " \times 0.25 "$} & 48 & 4 & 6 & 0.25 & 8 & 1 & 7395 & 7109.9 & $-3.9 \%$ \\
\hline & 48 & 4 & 6 & 0.25 & 8 & 1 & 6264 & 7109.9 & $13.5 \%$ \\
\hline & 48 & 4 & 6 & 0.25 & 8 & 1 & 6600 & 7109.9 & $7.7 \%$ \\
\hline & 57.5 & 4 & 6 & 0.25 & 9.6 & 1 & 6713 & 7252.2 & $8.0 \%$ \\
\hline \multirow[t]{4}{*}{$6 " \times 6 " \times 0.375^{\prime \prime}$} & 36 & 6 & 6 & 0.375 & 6 & 1 & 13397 & 11314.8 & $-15.5 \%$ \\
\hline & 36 & 6 & 6 & 0.375 & 6 & 1 & 12340 & 11314.8 & $-8.3 \%$ \\
\hline & 60 & 6 & 6 & 0.375 & 10 & 1 & 13231 & 13023.6 & $-1.6 \%$ \\
\hline & 60 & 6 & 6 & 0.375 & 10 & 1 & 12169 & 13023.6 & $7.0 \%$ \\
\hline
\end{tabular}

Note: Experimental loads highlighted in yellow were tested by Qureshi (2012)

\subsubsection{Results for $10<L / h \leq 42$}

Long span prediction has proven to be the most polished component of the prediction model. Several series of refinements have been made to Equation (3-10), which has been fit to over 50 lab tests, including multiple replications of test specimen sizes and spans within the applicable range of span-to-depth ratios $(10<L / h \leq 42)$. This equation produced a maximum flexural load capacity prediction error of $-22.3 \%$, with over $71 \%$ of all of the data points within $\pm 10 \%$ of the experimental value, as shown in Table 3-5 on the following page. The mean error 
for Equation (3-10) for the range $10<L / h \leq 42$ based on experimental data included in this study is $-3.0 \%$.

Here, much of the associated error of the equation can be attributed to imperfections in testing procedure, manufacturing inconsistencies, and the assumption that the characteristic longitudinal strength in the flange, $F_{L f}$, is consistently equal for all specimens based only on the manufacturer. In design, this value would be obtained from manufacturers' specifications based on coupon testing for each specimen size and type, thus resulting in greater accuracy and consistency in predicting flexural load capacity. 
Table 3-5 Flexural load capacity prediction results for box members where $10<L / h<42$ using Equation (3-10)

\begin{tabular}{|c|c|c|c|c|c|c|c|c|c|}
\hline \multirow{2}{*}{ Section } & \multirow[b]{2}{*}{ L (in) } & \multirow[b]{2}{*}{$b_{f}$ (in.) } & \multirow[b]{2}{*}{$h$ (in.) } & \multirow[b]{2}{*}{$t$ (in.) } & \multirow[b]{2}{*}{$\mathrm{L} / \mathrm{h}$} & \multirow[b]{2}{*}{$\begin{array}{l}\text { Number of } \\
\text { Replications }\end{array}$} & \multirow[b]{2}{*}{$\mathbf{P}_{\text {expt }}$ (lbf) } & \multicolumn{2}{|c|}{ Equation (3-10) } \\
\hline & & & & & & & & $\mathbf{P}_{\mathrm{cr}}(\mathrm{lbf})$ & \% Error \\
\hline \multirow{2}{*}{$3 " \times 3 " \times 0.25 "$} & $\overline{72}$ & 3 & 3 & 0.25 & 24 & 1 & 4033 & 44459.7 & $10.6 \%$ \\
\hline & 72 & 3 & 3 & 0.25 & 24 & 1 & 4280 & 4459.7 & $4.2 \%$ \\
\hline \multirow[t]{4}{*}{$3.5^{\prime \prime} \times 3.5^{\prime \prime} \times 0.25^{\prime \prime}$} & 36 & 3.5 & 3.5 & 0.25 & 10.3 & 3 & 6185 & 6267.5 & $1.3 \%$ \\
\hline & 60 & 3.5 & 3.5 & 0.25 & 17.1 & 3 & 4857 & 4854.8 & $0.0 \%$ \\
\hline & 108 & 3.5 & 3.5 & 0.25 & 30.9 & 3 & 3714 & 3618.5 & $-2.6 \%$ \\
\hline & 144 & 3.5 & 3.5 & 0.25 & 41.1 & 3 & 3137 & 3133.8 & $-0.1 \%$ \\
\hline \multirow[t]{4}{*}{$4^{\prime \prime} \times 4^{\prime \prime} \times 0.375^{\prime \prime}$} & 48 & 4 & 4 & 0.375 & 12 & 3 & 10875 & 9298.6 & $-14.5 \%$ \\
\hline & 84 & 4 & 4 & 0.375 & 21 & 3 & 9049 & 7029.1 & $-22.3 \%$ \\
\hline & 120 & 4 & 4 & 0.375 & 30 & 3 & 7540 & 5880.9 & $-22.0 \%$ \\
\hline & 168 & 4 & 4 & 0.375 & 42 & 3 & 5624 & 4970.3 & $-11.6 \%$ \\
\hline \multirow[t]{15}{*}{$6^{\prime \prime} \times 4^{\prime \prime} \times 0.25^{\prime \prime}$} & 72 & 4 & 6 & 0.25 & 12 & 1 & 5941 & 6150.9 & $3.5 \%$ \\
\hline & 72 & 4 & 6 & 0.25 & 12 & 1 & 7180 & 6150.9 & $-14.3 \%$ \\
\hline & 72 & 4 & 6 & 0.25 & 12 & 1 & 6319 & 6150.9 & $-2.7 \%$ \\
\hline & 84 & 4 & 6 & 0.25 & 14 & 1 & 5915 & 5694.6 & $-3.7 \%$ \\
\hline & 84 & 4 & 6 & 0.25 & 14 & 1 & 5981 & 5694.6 & $-4.8 \%$ \\
\hline & 84 & 4 & 6 & 0.25 & 14 & 1 & 6267 & 5694.6 & $-9.1 \%$ \\
\hline & 84 & 4 & 6 & 0.25 & 14 & 3 & 6019 & 5694.6 & $-5.4 \%$ \\
\hline & 105.6 & 4 & 6 & 0.25 & 17.6 & 1 & 5507 & 5078.9 & $-7.8 \%$ \\
\hline & 120 & 4 & 6 & 0.25 & 20 & 1 & 4738 & 4764.5 & $0.6 \%$ \\
\hline & 120 & 4 & 6 & 0.25 & 20 & 1 & 5022 & 4764.5 & $-5.1 \%$ \\
\hline & 120 & 4 & 6 & 0.25 & 20 & 3 & 5215 & 4764.5 & $-8.6 \%$ \\
\hline & 168 & 4 & 6 & 0.25 & 28 & 1 & 4355 & 4026.7 & $-7.5 \%$ \\
\hline & 168 & 4 & 6 & 0.25 & 28 & 1 & 4343 & 4026.7 & $-7.3 \%$ \\
\hline & 168 & 4 & 6 & 0.25 & 28 & 1 & 4665 & 4026.7 & $-13.7 \%$ \\
\hline & 168 & 4 & 6 & 0.25 & 28 & 3 & 4424 & 4026.7 & $-9.0 \%$ \\
\hline \multirow[t]{7}{*}{$6 " \times 6 " \times 0.375 "$} & 96 & 6 & 6 & 0.375 & 16 & 1 & 10048 & 10850.9 & $8.0 \%$ \\
\hline & 96 & 6 & 6 & 0.375 & 16 & 1 & 10101 & 10850.9 & $7.4 \%$ \\
\hline & 96 & 6 & 6 & 0.375 & 16 & 1 & 9688 & 10850.9 & $12.0 \%$ \\
\hline & 144 & 6 & 6 & 0.375 & 24 & 1 & 9080 & 8859.7 & $-2.4 \%$ \\
\hline & 144 & 6 & 6 & 0.375 & 24 & 1 & 8261 & 8859.7 & $7.2 \%$ \\
\hline & 180 & 6 & 6 & 0.375 & 30 & 1 & 7294 & 7924.4 & $8.6 \%$ \\
\hline & 180 & 6 & 6 & 0.375 & 30 & 1 & 6966 & 7924.4 & $13.8 \%$ \\
\hline
\end{tabular}

Note: Experimental loads highlighted in yellow were tested by Qureshi (2012) 


\subsection{Results from Existing Prediction Models}

The results from the November 2010 draft version of the ASCE/ACMA Pre-Standard for Load and Resistance Factor Design (LRFD) of Pultruded Fiber Reinforced Polymer (FRP) Structures are shown in Table 3-6. As previously mentioned, this model requires the application of equations for two failure modes, and the lesser of the two predicted critical flexural capacities is to be taken as the design capacity. For direct comparison, the controlling critical buckling stress is converted to the critical flexural capacity using Equation (3-9), where $M=P L / 4$ for three-point bending. Based on this same equation for the maximum moment under three-point bending, the nominal flexural strength, $M_{n}$, is converted to the critical flexural capacity by multiplying by $4 / L$. It should be noted that when applying this model to the square and rectangular box members included in this study, Equation (3-16) was always the controlling design equation, as can be seen in the table.

A second set of results, from the May 2013 draft version of the ASCE/ACMA PreStandard for Load and Resistance Factor Design (LRFD) of Pultruded Fiber Reinforced Polymer (FRP) Structures, is shown in Table 3-7. This model requires only one mode of failure to be checked for the nominal flexural strength of square and rectangular box members based on the minimum values of local buckling in the compression flange and web local buckling, calculated using Equation (3-25) and Equation (3-26), respectively. When applying this model to the square and rectangular box members included in this study, the critical buckling stress of the compression flange always controlled the design. This controlling critical buckling stress is then converted to the critical flexural capacity using Equation (3-9), where $M=P L / 4$ for three-point bending. All loads highlighted in yellow in Table 3-6 and Table 3-7 were tested by Qureshi (2012). 
Table 3-6 Results for critical flexural capacity prediction model from Nov. 2010 draft of LRFD Pre-Standard

\begin{tabular}{|c|c|c|c|c|c|c|c|c|}
\hline \multirow{2}{*}{ Sample } & \multirow[b]{2}{*}{ L (in) } & \multirow[b]{2}{*}{$\mathbf{P}_{\text {expt }}(\mathrm{lbf})$} & \multicolumn{2}{|c|}{ Eq. (3-16) } & \multicolumn{2}{|c|}{ Eq. (3-19) } & \multicolumn{2}{|c|}{$\operatorname{Min}(3-16,3-19)$} \\
\hline & & & $M_{n}$ (lbfoin.) & $\mathbf{P}_{\mathrm{cr}}(\mathrm{lbf})$ & $\mathrm{f}_{\mathrm{cr}}(\mathrm{psi})$ & $\mathbf{P}_{\mathrm{cr}}(\mathrm{lbf})$ & $\mathbf{P}_{\mathrm{cr}}(\mathrm{lbf})$ & $\%$ Error \\
\hline \multirow{8}{*}{$3 " \times 3 " \times 0.25 "$} & 15 & 7931 & 113464 & 30257 & 259772 & 161395 & 30257 & $281.5 \%$ \\
\hline & 18 & 5749 & 113464 & 25214 & 259772 & 134496 & 25214 & $338.6 \%$ \\
\hline & 18 & 6177 & 113464 & 25214 & 259772 & 134496 & 25214 & $308.2 \%$ \\
\hline & 18 & 6015 & 113464 & 25214 & 259772 & 134496 & 25214 & $319.2 \%$ \\
\hline & 30 & 6187 & 113464 & 15129 & 259772 & 80698 & 15129 & $144.5 \%$ \\
\hline & 30 & 4783 & 113464 & 15129 & 259772 & 80698 & 15129 & $216.3 \%$ \\
\hline & 72 & 4033 & 113464 & 6304 & 259772 & 33624 & 6304 & $56.3 \%$ \\
\hline & 72 & 4280 & 113464 & 6304 & 259772 & 33624 & 6304 & $47.3 \%$ \\
\hline \multirow[t]{4}{*}{$3.5^{\prime \prime} \times 3.5^{\prime \prime} \times 0.25^{\prime \prime}$} & 36 & 6185 & 131547 & 14616 & 156261 & 57099 & 14616 & $136.3 \%$ \\
\hline & 60 & 4857 & 131547 & 8770 & 156261 & 34260 & 8770 & $80.6 \%$ \\
\hline & 108 & 3714 & 131547 & 4872 & 156261 & 19033 & 4872 & $31.2 \%$ \\
\hline & 144 & 3137 & 131547 & 3654 & 156261 & 14275 & 3654 & $16.5 \%$ \\
\hline \multirow[t]{4}{*}{$4 " \times 4 " \times 0.375 "$} & 48 & 10875 & 240723 & 20060 & 269185 & 134998 & 20060 & $84.5 \%$ \\
\hline & 84 & 9049 & 240723 & 11463 & 269185 & 77142 & 11463 & $26.7 \%$ \\
\hline & 120 & 7540 & 240723 & 8024 & 269185 & 53999 & 8024 & $6.4 \%$ \\
\hline & 168 & 5624 & 240723 & 5732 & 269185 & 38571 & 5732 & $1.9 \%$ \\
\hline \multirow[t]{21}{*}{$6 " \times 4 " \times 0.25 "$} & 24 & 5783 & 312986 & 52164 & 109368 & 142628 & 52164 & $802.0 \%$ \\
\hline & 30 & 6178 & 312986 & 41731 & 109368 & 114102 & 41731 & $575.5 \%$ \\
\hline & 48 & 7395 & 312986 & 26082 & 109368 & 71314 & 26082 & $252.7 \%$ \\
\hline & 48 & 6264 & 312986 & 26082 & 109368 & 71314 & 26082 & $316.4 \%$ \\
\hline & 48 & 6600 & 312986 & 26082 & 109368 & 71314 & 26082 & $295.2 \%$ \\
\hline & 57.5 & 6713 & 312986 & 21773 & 109368 & 59532 & 21773 & $224.3 \%$ \\
\hline & 72 & 5941 & 312986 & 17388 & 109368 & 47543 & 17388 & $192.7 \%$ \\
\hline & 72 & 7180 & 312986 & 17388 & 109368 & 47543 & 17388 & $142.2 \%$ \\
\hline & 72 & 6319 & 312986 & 17388 & 109368 & 47543 & 17388 & $175.2 \%$ \\
\hline & 84 & 5915 & 312986 & 14904 & 109368 & 40751 & 14904 & $152.0 \%$ \\
\hline & 84 & 5981 & 312986 & 14904 & 109368 & 40751 & 14904 & $149.2 \%$ \\
\hline & 84 & 6267 & 312986 & 14904 & 109368 & 40751 & 14904 & $137.8 \%$ \\
\hline & 84 & 6019 & 312986 & 14904 & 109368 & 40751 & 14904 & $147.6 \%$ \\
\hline & 105.6 & 5507 & 312986 & 11856 & 109368 & 32415 & 11856 & $115.3 \%$ \\
\hline & 120 & 4738 & 312986 & 10433 & 109368 & 28526 & 10433 & $120.2 \%$ \\
\hline & 120 & 5022 & 312986 & 10433 & 109368 & 28526 & 10433 & $107.7 \%$ \\
\hline & 120 & 5215 & 312986 & 10433 & 109368 & 28526 & 10433 & $100.1 \%$ \\
\hline & 168 & 4355 & 312986 & 7452 & 109368 & 20375 & 7452 & $71.1 \%$ \\
\hline & 168 & 4343 & 312986 & 7452 & 109368 & 20375 & 7452 & $71.6 \%$ \\
\hline & 168 & 4665 & 312986 & 7452 & 109368 & 20375 & 7452 & $59.7 \%$ \\
\hline & 168 & 4424 & 312986 & 7452 & 109368 & 20375 & 7452 & $68.4 \%$ \\
\hline \multirow[t]{12}{*}{$6 " \times 6 " \times 0.375 "$} & 30 & 11153 & 595898 & 79453 & 119638 & 237640 & 79453 & $612.4 \%$ \\
\hline & 36 & 13397 & 595898 & 66211 & 119638 & 198034 & 66211 & $394.2 \%$ \\
\hline & 36 & 12340 & 595898 & 66211 & 119638 & 198034 & 66211 & $436.6 \%$ \\
\hline & 60 & 13231 & 595898 & 39727 & 119638 & 118820 & 39727 & $200.3 \%$ \\
\hline & 60 & 12169 & 595898 & 39727 & 119638 & 118820 & 39727 & $226.5 \%$ \\
\hline & 96 & 10048 & 595898 & 24829 & 119638 & 74263 & 24829 & $147.1 \%$ \\
\hline & 96 & 10101 & 595898 & 24829 & 119638 & 74263 & 24829 & $145.8 \%$ \\
\hline & 96 & 9688 & 595898 & 24829 & 119638 & 74263 & 24829 & $156.3 \%$ \\
\hline & 144 & 9080 & 595898 & 16553 & 119638 & 49508 & 16553 & $82.3 \%$ \\
\hline & 144 & 8261 & 595898 & 16553 & 119638 & 49508 & 16553 & $100.4 \%$ \\
\hline & 180 & 7294 & 595898 & 13242 & 119638 & 39607 & 13242 & $81.5 \%$ \\
\hline & 180 & 6966 & 595898 & 13242 & 119638 & 39607 & 13242 & $90.1 \%$ \\
\hline
\end{tabular}


Table 3-7 Results for critical flexural capacity prediction model from May 2013 draft of LRFD Pre-Standard

\begin{tabular}{|c|c|c|c|c|c|c|}
\hline & & & Eq. (3-25) & Eq. (3-24) & & \\
\hline Sample & L (in) & $\mathbf{P}_{\text {expt }}$ (lbf) & $\mathrm{F}_{\mathrm{crf}}(\mathrm{psi})$ & $M_{n}$ (lbf•in.) & $P_{\text {cr }}(\mathbf{l b f})$ & $\%$ Error \\
\hline \multirow{8}{*}{$3 " \times 3 " \times 0.25 "$} & 15 & $\overline{77931}$ & $\bar{~} 51186$ & 1119257 & 31802 & $301.0 \%$ \\
\hline & 18 & 5749 & 51186 & 119257 & 26501 & $361.0 \%$ \\
\hline & 18 & 6177 & 51186 & 119257 & 26501 & $329.0 \%$ \\
\hline & 18 & 6015 & 51186 & 119257 & 26501 & $340.6 \%$ \\
\hline & 30 & 6187 & 51186 & 119257 & 15901 & $157.0 \%$ \\
\hline & 30 & 4783 & 51186 & 119257 & 15901 & $232.4 \%$ \\
\hline & 72 & 4033 & 51186 & 119257 & 6625 & $64.3 \%$ \\
\hline & 72 & 4280 & 51186 & 119257 & 6625 & $54.8 \%$ \\
\hline \multirow[t]{4}{*}{$3.5^{\prime \prime} \times 3.5^{\prime \prime} \times 0.25^{\prime \prime}$} & 36 & 6185 & 30493 & 100281 & 11142 & $80.2 \%$ \\
\hline & 60 & 4857 & 30493 & 100281 & 6685 & $37.6 \%$ \\
\hline & 108 & 3714 & 30493 & 100281 & 3714 & $0.0 \%$ \\
\hline & 144 & 3137 & 30493 & 100281 & 2786 & $-11.2 \%$ \\
\hline \multirow{4}{*}{$4^{\prime \prime} \times 4 " \times 0.375^{\prime \prime}$} & 48 & 10875 & 52528 & 316120 & 26343 & $142.2 \%$ \\
\hline & 84 & 9049 & 52528 & 316120 & 15053 & $66.4 \%$ \\
\hline & 120 & 7540 & 52528 & 316120 & 10537 & $39.8 \%$ \\
\hline & 168 & 5624 & 52528 & 316120 & 7527 & $33.8 \%$ \\
\hline \multirow{21}{*}{$6 " \times 4 " \times 0.25 "$} & 24 & 5783 & 23346 & 182674 & 30446 & $426.5 \%$ \\
\hline & 30 & 6178 & 23346 & 182674 & 24357 & $294.2 \%$ \\
\hline & 48 & 7395 & 23346 & 182674 & 15223 & $105.9 \%$ \\
\hline & 48 & 6264 & 23346 & 182674 & 15223 & $143.0 \%$ \\
\hline & 48 & 6600 & 23346 & 182674 & 15223 & $130.6 \%$ \\
\hline & 57.5 & 6713 & 23346 & 182674 & 12708 & $89.3 \%$ \\
\hline & 72 & 5941 & 23346 & 182674 & 10149 & $70.8 \%$ \\
\hline & 72 & 7180 & 23346 & 182674 & 10149 & $41.3 \%$ \\
\hline & 72 & 6319 & 23346 & 182674 & 10149 & $60.6 \%$ \\
\hline & 84 & 5915 & 23346 & 182674 & 8699 & $47.1 \%$ \\
\hline & 84 & 5981 & 23346 & 182674 & 8699 & $45.4 \%$ \\
\hline & 84 & 6267 & 23346 & 182674 & 8699 & $38.8 \%$ \\
\hline & 84 & 6019 & 23346 & 182674 & 8699 & $44.5 \%$ \\
\hline & 105.6 & 5507 & 23346 & 182674 & 6919 & $25.6 \%$ \\
\hline & 120 & 4738 & 23346 & 182674 & 6089 & $28.5 \%$ \\
\hline & 120 & 5022 & 23346 & 182674 & 6089 & $21.2 \%$ \\
\hline & 120 & 5215 & 23346 & 182674 & 6089 & $16.8 \%$ \\
\hline & 168 & 4355 & 23346 & 182674 & 4349 & $-0.1 \%$ \\
\hline & 168 & 4343 & 23346 & 182674 & 4349 & $0.1 \%$ \\
\hline & 168 & 4665 & 23346 & 182674 & 4349 & $-6.8 \%$ \\
\hline & 168 & 4424 & 23346 & 182674 & 4349 & $-1.7 \%$ \\
\hline \multirow[t]{12}{*}{$6 " \times 6 " \times 0.375 "$} & 30 & 11153 & 23346 & 347796 & 46373 & $315.8 \%$ \\
\hline & 36 & 13397 & 23346 & 347796 & 38644 & $188.5 \%$ \\
\hline & 36 & 12340 & 23346 & 347796 & 38644 & $213.2 \%$ \\
\hline & 60 & 13231 & 23346 & 347796 & 23186 & $75.2 \%$ \\
\hline & 60 & 12169 & 23346 & 347796 & 23186 & $90.5 \%$ \\
\hline & 96 & 10048 & 23346 & 347796 & 14491 & $44.2 \%$ \\
\hline & 96 & 10101 & 23346 & 347796 & 14491 & $43.5 \%$ \\
\hline & 96 & 9688 & 23346 & 347796 & 14491 & $49.6 \%$ \\
\hline & 144 & 9080 & 23346 & 347796 & 9661 & $6.4 \%$ \\
\hline & 144 & 8261 & 23346 & 347796 & 9661 & $16.9 \%$ \\
\hline & 180 & 7294 & 23346 & 347796 & 7729 & $6.0 \%$ \\
\hline & 180 & 6966 & 23346 & 347796 & 7729 & $11.0 \%$ \\
\hline
\end{tabular}


As exhibited by Table 3-6 and Table 3-7, both of the analyzed models from draft versions of the ASCE/ACMA LRFD Pre-Standard are highly inconsistent. The November 2010 model showed very little accuracy, predicting with 20 percent of the experimental critical flexural capacity for only two data points. The mean error percentage for this model is +184.6 percent based on the experimental data collected for this study. The May 2013 model showed fairly accurate prediction at high $L / h$ ratios, but proved to be inaccurate at shorter spans. The mean error percentage for this newer model is +100.2 percent based on the experimental data.

\subsection{Conclusions and Recommendations}

Compared with previous models shown in draft versions of the ASCE/ACMA PreStandard for Load and Resistance Factor Design (LRFD) of Pultruded Fiber Reinforced Polymer (FRP) Structures, the model proposed by the author for predicting the critical flexural capacity of pultruded GFRP square and rectangular box members in Section 3.3 has shown superior accuracy. Whereas the November 2010 and May 2013 ASCE/ACMA LRFD Pre-Standard prediction models exhibit average error percentages of +184.6 percent and +100.2 percent, respectively, the mean error for the proposed prediction model across all failure modes is calculated to be -2.06 percent. The proposed model also exhibits a standard deviation of the percent error across all test specimens of 9.153 percent, thus implying that there is very little dispersion from the mean error percentage, indicating excellent consistency. This model is also very simplistic and easy to apply. Based on the $L / h$ ratio, the designer is easily able to identify one equation which will be provide the flexural design load of a pultruded GFRP box member for use as a structural component. Overall, the proposed prediction model exhibits excellent accuracy and consistency, as well as simplicity, in comparison to the models found in previous 
draft versions of the ASCE/ACMA Pre-Standard for Load and Resistance Factor Design (LRFD) of Pultruded Fiber Reinforced Polymer (FRP) Structures. The simple but effective nature of this model makes it highly suitable for use in a design code with applicable resistance factors applied.

Continued testing and analysis is being carried out at the West Virginia University Constructed Facilities Center (WVU-CFC) in order to further refine the prediction model. Due to the limited amount of critical flexural load capacity data within the range $4 \leq L / h<6$, extensive testing is required to confirm the applicability of the local buckling in the compression flange failure mode. Additional testing is also needed within the range $6 \leq L / h \leq 10$ to confirm the influence and orientation angle of the principal strains. The bending failure in the tension flange failure mode for the range $10<L / h \leq 42$ is the most refined equation included in the prediction model, having been fit with good accuracy to a large number of replications, and therefore does not require any further development.

As evidenced by Figure 3-7, Figure 3-9, and Figure 3-10, the stress intensity factor works well with the prediction model, but further investigation into the mechanics of the equation may be needed to prove its applicability to pultruded FRP box beams under transverse load conditions. At this point, the derivation shown in Appendix B serves as the theoretical basis for this stress intensity factor.

Lastly, further testing at the coupon level is needed to ensure the accuracy of the prediction model. For this study, values for the strength and stiffness properties of the sections were based on average values for each manufacturer obtained from a limited number of coupon tension testing. In design practice, values for these properties would be provided for each specimen size and type by the manufacturer. Better precision in the laminate properties should result in greater accuracy in prediction of the critical flexural load capacity. 


\section{CHAPTER 4 LATERAL-TORSIONAL BUCKLING LOAD PREDICTION FOR OPEN SECTIONS}

\subsection{Introduction and Scope}

Lateral-torsional buckling (LTB) is one of several limit states that has to be satisfied in the design of open cross section beams, even with lateral supports spaced at certain distances along the beam span length. Commonly known as open sections, these beams include wide flange (WF) sections, channels, and angles, among others. LTB is a type of geometric instability which develops in the compression zone of a transversely loaded beam at a critical load (Popov 1954). As the load approaches this limit, the compression flange begins to buckle laterally and the web begins to twist, leading to torsion moving the beam out of its vertical plane (Bank 2006). The resulting failure mode is commonly referred to as lateral-torsional buckling. A failure mode of this type cannot occur when the moment of inertia about the major bending axis is less than or equal to the out-of-plane moment of inertia, i.e., $I_{x} \leq I_{y}$ (AISC 2001). The issue of LTB is especially important in the case of pultruded glass fiber-reinforced polymer (GFRP) beams possessing a low modulus of rigidity (about 8 to 10 times lower than steel), where thin-walled profiles tend to be slender. The LTB limit state is generally addressed by providing lateral bracing along the length of the member.

The objective of this research is to (1) acquire data from three-point bend testing of pultruded GFRP channel and WF sections in a variety of sizes, (2) determine the critical buckling load of these specimens, and (3) develop an accurate prediction model for the critical buckling load. Testing was performed for beam spans using multiple kinds of lateral bracing mechanisms placed at several different bracing positions along the test specimen span lengths. 
Due to some variation in data plots, subsequent analysis of this data led to the development of a critical buckling load range defined by an upper and lower bound. Multiple LTB equations from several sources were considered and applied but yielded inaccurate results, leading to the development of a new critical buckling load prediction model by the author.

\subsection{Samples and Test Procedure}

The beams in this study ranged from 6 inches to 12 inches in depth for wide flange (WF) sections and from 4 inches to 14 inches in depth for channel specimens. WF section flange widths ranged from 4 inches to 12 inches, and channel legs ranged from 1.125 inches to 3.5 inches. Wall thicknesses ranged from 0.375 to 0.5 inches for WF sections, and from 0.1875 inches to 0.75 inches for channel specimens. This covers a wide range of sizes of commonly produced pultruded GFRP open sections. The beams were constructed from E-glass fibers and mats with vinyl ester resin used as a binder. Three-point bend tests were performed on the samples over spans ranging from 72 inches to 108 inches. The majority of this study focuses on samples tested at a span of 108 inches, unless otherwise noted. A summary of testing is provided in Table 4-1 and Table 4-2, with the nomenclature for the given dimensions explained by Figure $4-1$.

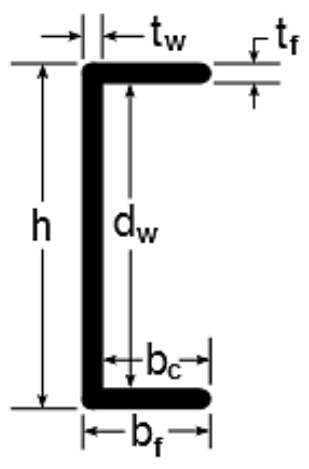

(a)

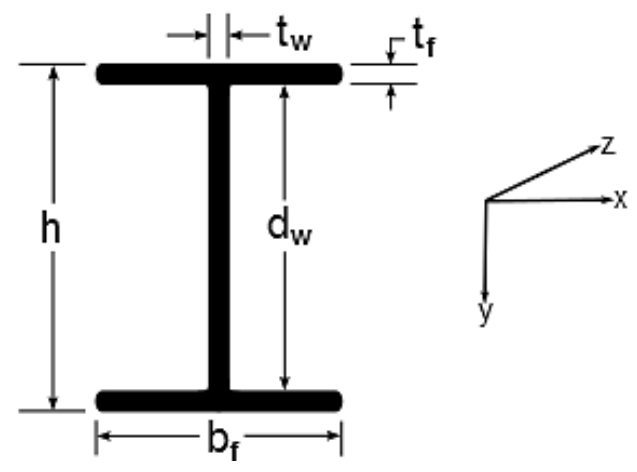

(b)

Figure 4-1 Dimensions of (a) channel section and (b) WF section used in the proposed model 
Table 4-1 Cross-sectional dimensions of samples tested for analysis of WF sections

\begin{tabular}{|c|c|c|c|c|c|c|c|c|}
\hline Sample & $\mathbf{L}$ (in.) & $\mathbf{L}_{\mathbf{b}}$ (in.) & $\mathbf{t}_{\mathrm{w}}$ (in.) & $\mathbf{t}_{\mathbf{f}}$ (in.) & $\mathbf{b}_{\mathbf{f}}$ (in.) & $\mathbf{h}$ (in.) & $\mathbf{d}_{\mathbf{w}}$ (in.) & Area $\left(\mathrm{in}^{2}\right)$ \\
\hline $12 " \times 12 " \times 0.5 " *$ & 180 & $\begin{array}{c}-- \\
-\end{array}$ & 0.5 & 0.5 & 12 & 12 & 11 & 17.5 \\
\hline $10^{\prime \prime} \times 5 " \times 0.5 "$ & 108 & 60 & 0.5 & 0.5 & 5 & 10 & 9 & 9.5 \\
\hline $8^{\prime \prime} \times 8^{\prime \prime} \times 0.375^{\prime \prime}$ & 108 & 60 & 0.375 & 0.375 & 8 & 8 & 7.25 & 8.71875 \\
\hline $8^{\prime \prime} \times 4^{\prime \prime} \times 0.375^{\prime \prime}$ & 72 & 36 & 0.375 & 0.375 & 4 & 8 & 7.25 & 5.71875 \\
\hline $6 " \times 6 " \times 0.375 "$ & 96 & 48 & 0.375 & 0.375 & 6 & 6 & 5.25 & 6.46875 \\
\hline
\end{tabular}

* tested under four-point bending by Bendidi (1996) (discussed later)

Table 4-2 Cross-sectional dimensions of samples tested for analysis of channel sections

\begin{tabular}{|c|c|c|c|c|c|c|c|c|c|}
\hline Sample & $\mathbf{L}$ (in.) & $\mathbf{L}_{\mathbf{b}}$ (in.) & $\mathbf{t}_{\mathrm{w}}$ (in.) & $\mathbf{t}_{\mathbf{f}}$ (in.) & $\mathbf{b}_{\mathbf{f}}$ (in.) & $\mathbf{b}_{\mathbf{c}}$ (in.) & $\mathbf{h}$ (in.) & $\mathbf{d}_{\mathrm{w}}$ (in.) & Area $\left(\mathrm{in}^{2}\right)$ \\
\hline $14^{\prime \prime} \times 3.5^{\prime \prime} \times 0.75^{\prime \prime}$ & 108 & 60 & 0.75 & 0.75 & 3.5 & 2.75 & 14 & 12.5 & 14.625 \\
\hline $10^{\prime \prime} \times 2.75 " \times 0.5 "$ & 108 & 60 & 0.5 & 0.5 & 2.75 & 2.25 & 10 & 9 & 7.250 \\
\hline $8^{\prime \prime} \times 2.25^{\prime \prime} \times 0.375^{\prime \prime}$ & 108 & 60 & 0.375 & 0.375 & 2.25 & 1.875 & 8 & 7.25 & 4.406 \\
\hline $6 " \times 1.625 " \times 0.1875 "$ & 108 & 60 & 0.1875 & 0.1875 & 1.625 & 1.4375 & 6 & 5.625 & 1.664 \\
\hline $5.5^{\prime \prime} \times 1.5^{\prime \prime} \times 0.25^{\prime \prime}$ & 108 & 60 & 0.25 & 0.25 & 1.5 & 1.25 & 5.5 & 5 & 2.000 \\
\hline $4 " \times 1.125 " \times 0.1875 "$ & 108 & 60 & 0.1875 & 0.1875 & 1.125 & 0.9375 & 4 & 3.625 & 1.102 \\
\hline
\end{tabular}

Application of the model proposed herein requires the laminate properties of the tested sections. These values are based on average values from tension coupon testing, as discussed in Appendix C, as well as values reported by Bendidi (1996). Based on observation of this data, the transverse modulus was taken to be equal to the longitudinal elastic modulus divided by 2.45 for open sections. In actual design practices, these values would be obtained through catalogued strength and stiffness properties for each sections provided by the manufacturer. The laminate properties for GFRP open sections included in this study can be seen in Table 4-3 and Table 4-4. It should be noted that this study assumes elastic modulus values in the flange do not differ from those in the web. 
Table 4-3 Laminate properties for WF sections included in this study from tension coupon testing

\begin{tabular}{|c|c|c|c|c|c|}
\hline Sample & $\mathbf{E}_{\mathrm{L}, \mathrm{f}}(\mathbf{M s i})$ & $\mathbf{E}_{\mathrm{L}, \mathrm{w}}(\mathrm{Msi})$ & $\mathrm{E}_{\mathrm{T}, \mathrm{f}}(\mathrm{Msi})$ & $\mathbf{E}_{\mathrm{T}, \mathrm{w}}(\mathbf{M s i})$ & $\mathbf{G}_{\mathrm{LT}}$ (Msi) \\
\hline $12^{\prime \prime} \times 12^{\prime \prime} \times 0.5 " *$ & 4.235 & 4.235 & 1.729 & 1.729 & 0.761 \\
\hline $10^{\prime \prime} \times 5^{\prime \prime} \times 0.5^{\prime \prime}$ & 5.120 & 5.120 & 2.090 & 2.090 & 0.490 \\
\hline $8 " \times 8 " \times 0.375 "$ & 5.120 & 5.120 & 2.090 & 2.090 & 0.490 \\
\hline $8^{\prime \prime} \times 4^{\prime \prime} \times 0.375^{\prime \prime}$ & 5.120 & 5.120 & 2.090 & 2.090 & 0.490 \\
\hline $6 " \times 6 " \times 0.375 "$ & 5.120 & 5.120 & 2.090 & 2.090 & 0.490 \\
\hline
\end{tabular}

* tested under four-point bending by Bendidi (1996) (discussed later)

Table 4-4 Laminate properties for channel sections included in this study from tension coupon testing

\begin{tabular}{|c|c|c|c|c|c|}
\hline Sample & $\mathbf{E}_{\mathbf{L}, \mathbf{f}}$ (Msi) & $\mathbf{E}_{\mathrm{L}, \mathrm{w}}(\mathbf{M s i})$ & $\mathbf{E}_{\mathrm{T}, \mathrm{f}}$ (Msi) & $\mathbf{E}_{\mathrm{T}, \mathrm{w}}(\mathbf{M s i})$ & $\mathbf{G}_{\mathrm{LT}}$ (Msi) \\
\hline $14 " \times 3.5^{\prime \prime} \times 0.75^{\prime \prime}$ & 4.30 & 4.30 & 1.755 & 1.755 & 0.42 \\
\hline $10^{\prime \prime} \times 2.75^{\prime \prime} \times 0.5 "$ & 4.30 & 4.30 & 1.755 & 1.755 & 0.42 \\
\hline $8^{\prime \prime} \times 2.25^{\prime \prime} \times 0.375^{\prime \prime}$ & 5.12 & 5.12 & 2.090 & 2.090 & 0.49 \\
\hline $6 " \times 1.625 " \times 0.1875 "$ & 5.12 & 5.12 & 2.090 & 2.090 & 0.49 \\
\hline $5.5^{\prime \prime} \times 1.5^{\prime \prime} \times 0.25^{\prime \prime}$ & 4.30 & 4.30 & 1.755 & 1.755 & 0.42 \\
\hline $4 " \times 1.125 " \times 0.1875 "$ & 5.12 & 5.12 & 2.090 & 2.090 & 0.49 \\
\hline
\end{tabular}

All samples were equipped with strain gages having a $1 / 4$ inch gage length which were installed prior to setting up the test. In addition, all channel specimens were fitted with a steel angle attached to the outer web, level with the top of the specimen in order to accommodate loading at the shear center, i.e., just outside (less than one inch) of the outer face of the web of the section. Figure 4-2 shows the approximate location of the shear center and center of gravity for WF and channel sections.

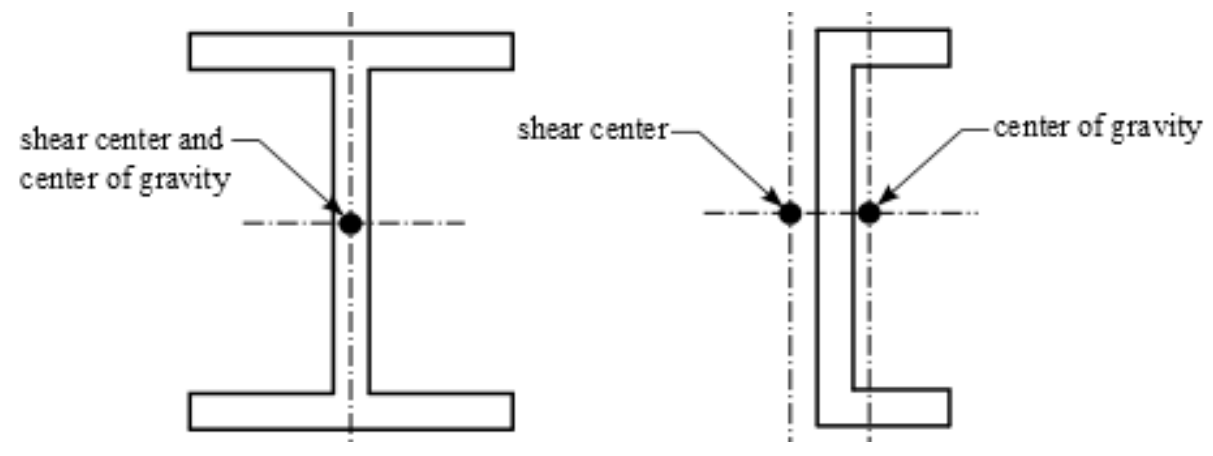

Figure 4-2 Approximate locations of shear center and center of gravity for WF and channel sections. 
The leg of the steel angle attached to the web was cut to a height equal to one-third of the total depth of the specimen, up to a maximum of 6 inches. The other leg of the angle was cut to a length sufficiently long enough to accommodate the load cell centered above the shear center. These angles were attached to the outer web using Pliogrip structural adhesive manufactured by Ashland Inc., a specialty chemical company. A 1 " $\times 1$ " grid of holes was drilled into the angle, and horizontal grooves were ground between the rows of holes to improve bond strength and adhesion. The surface area of the web where the angle was to be attached was also slightly roughened using sand paper. Figure 4-3a shows the placement of the steel angle attached to the outer web of an $8^{\prime \prime} \times 2.25^{\prime \prime} \times 0.375^{\prime \prime}$ channel specimen.

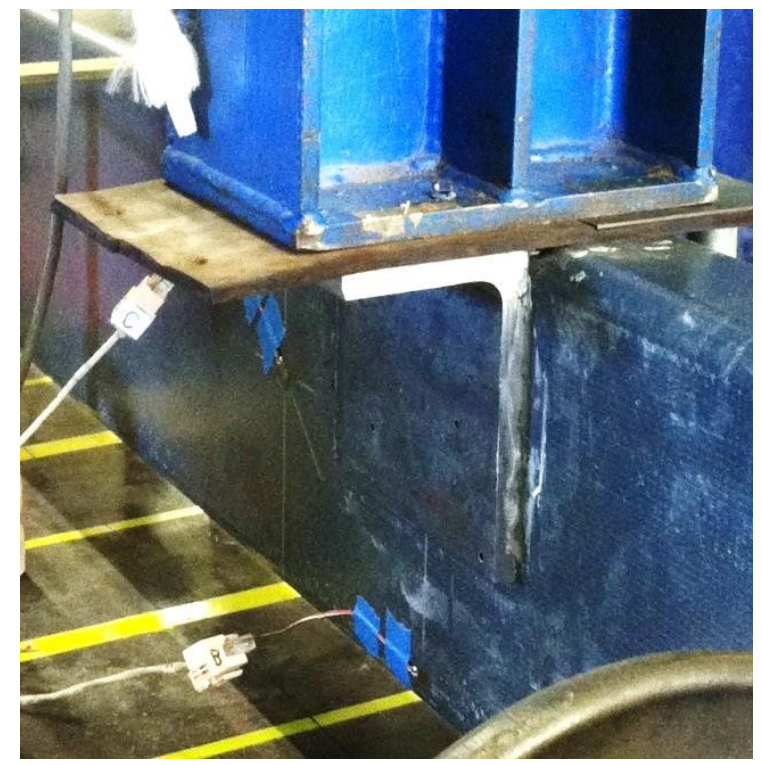

(a)

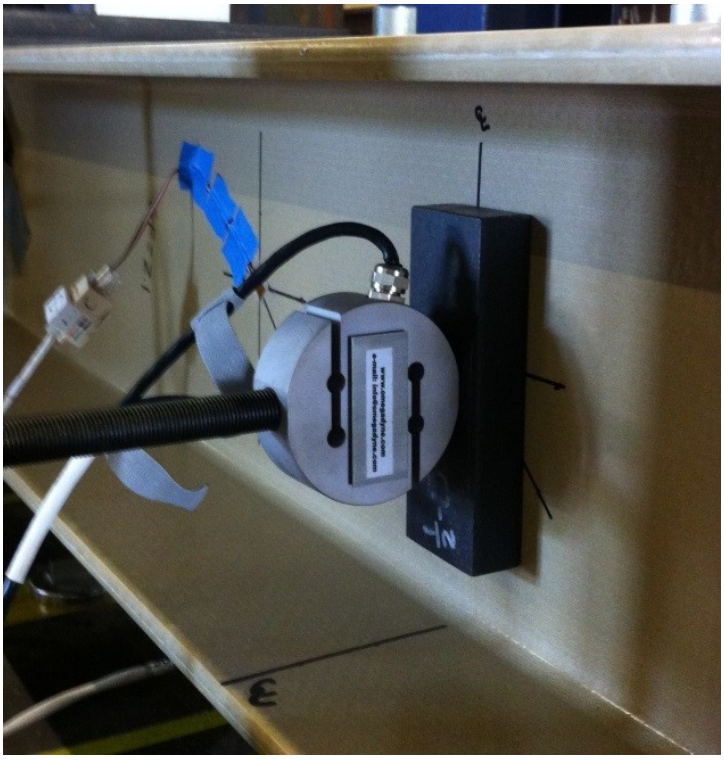

(b)

Figure 4-3 (a) Steel angle attached to the web of a 8" $\times 2.25 " \times 0.375 "$ channel using Pliogrip adhesive and (b) 1 kip load cell used to capture horizontal resistance provided by lateral bracing

Data collected from tests performed with loading at the shear center is not considered in this report. In theory, shear center loading should result in pure bending (i.e., zero torsion). Although pure bending was never achieved during testing, the observed effect of LTB was 
somewhat less when compared to loading at the center of gravity. Loading at the center of gravity represents a more critical load scenario, and therefore was used in the development of the prediction model.

The support conditions were steel roller-type supports at the ends with lateral constraints set up to allow warping and vertical rotation about the roller, but to restrain the beam end from twisting. Lateral constraints were provided by steel bar cut to about 65 percent of the depth of the web, secured firmly to the web using threaded rod attached to a fixed steel angle using nuts. Wooden lateral constraints bracing the outside edges of the flanges from moving were also considered, but found to be ineffective, as discussed in Section 4.8.4.

The load was applied at the center of the span by a hydraulic ram. The load was applied manually using a hand pump, and therefore loading rate could not be held constant throughout testing. The applied load was distributed uniformly over the entire width of the flange by a $1 / 4$ inch thick steel plate, with ultra-strength neoprene rubber elastomeric bearing padding having durometer hardness $50 \mathrm{~A}$ between the steel plate and the flange. The loading plate and elastomeric padding were aligned with the centerline of the specimen during all tests. The length of the steal load distribution plate was typically 6 inches for all tests.

The test set-up included five spring loaded LVDTs (RDP: DCTH400AG, Range \pm 0.4 in.) placed horizontally along the web during testing in hopes of capturing the point at which warping initiates, as shown in Figure 4-4. Three additional LVDTs were used to record the vertical deflection. One of these LVDTs was used to record the overall deflection at midspan of the tension flange (RDP: HDC-DCTH2000, Range \pm 2 in.), and two others, each attached to opposite ends of a GFRP bar overhanging both sides of the compression flange (Schavitz/MS: 3000 HCD, Range \pm 3 in.), as shown in Figure 4-5. By placing a vertical LVDT on each side of 
the top flange, lateral displacement of the flange could be identified by observing a divergence in plotted load versus deflection data. Two one kip tension-compression load cells (Omega LCR$1 \mathrm{~K})$ were used at the lateral supports to determine the horizontal resistance absorbed by the lateral bracing mechanism with increasing load, as shown in Figure 4-3b. A third load cell was used to record the value of the transverse load as it was applied. Here, a 10 kip load cell (Omega LC101-10K) was used when testing smaller sections, and a 40 kip load cell (Omega LC10140K) was used when testing larger sections. Strain gages having a $1 / 4$ inch gage length were installed on each specimen to record longitudinal strain in the tension flange of WF sections, and in the tension zone of the web near the web-flange junction for channel sections. Additional strain gages were installed at a 45 degree angle at mid-depth of the web at locations at or near the midspan, and also 2.5 feet away from the midspan. All data was continuously recorded using a StrainSmart computer program utilizing a Vishay System 7000 data acquisition system during each of the trials.

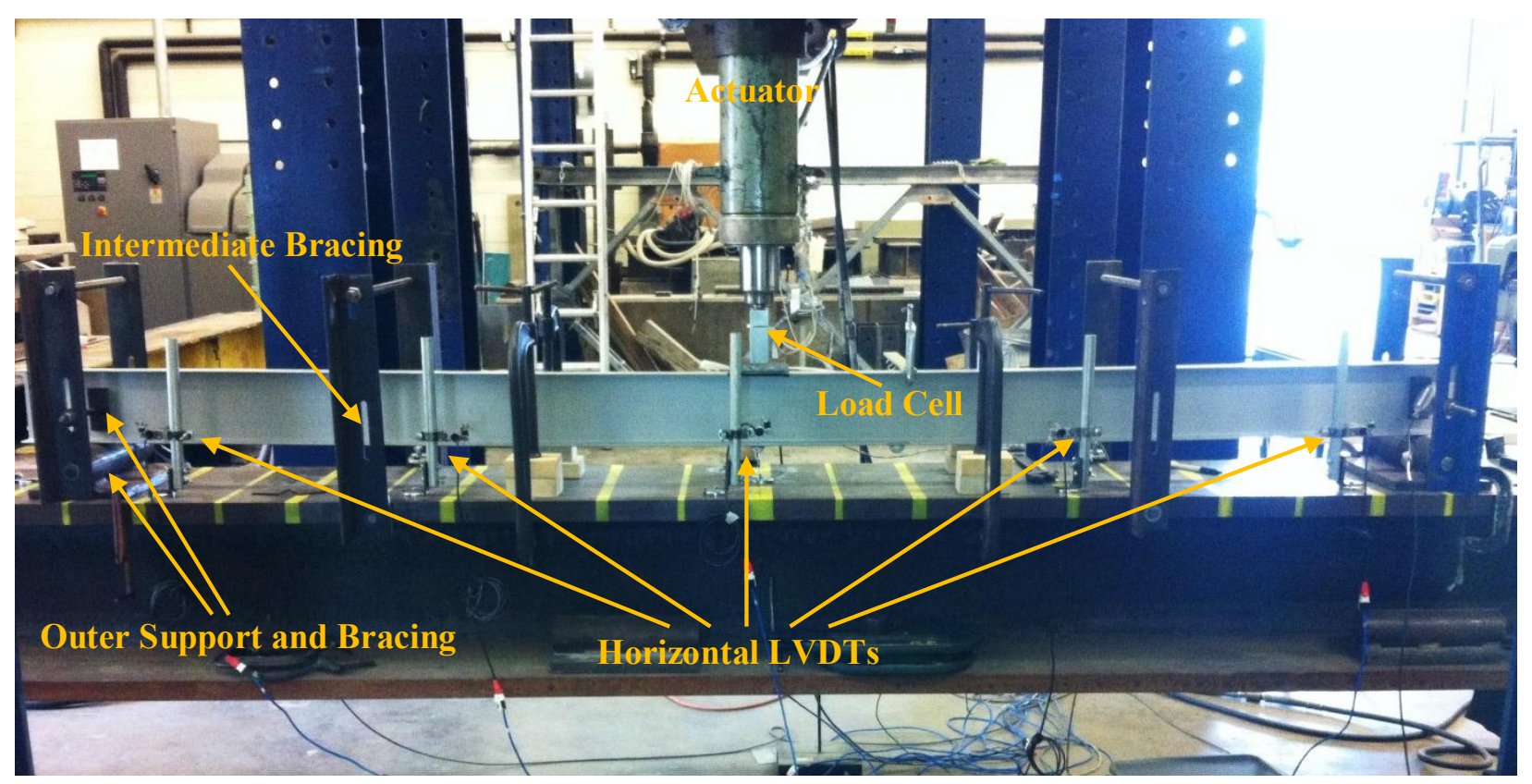

Figure 4-4 Front view of test setup for 6 " $\times 1.625$ " $\times 0.1875$ " channel section subjected to three-point bending with intermediate lateral brace points $2.5 \mathrm{ft}$. from midspan 


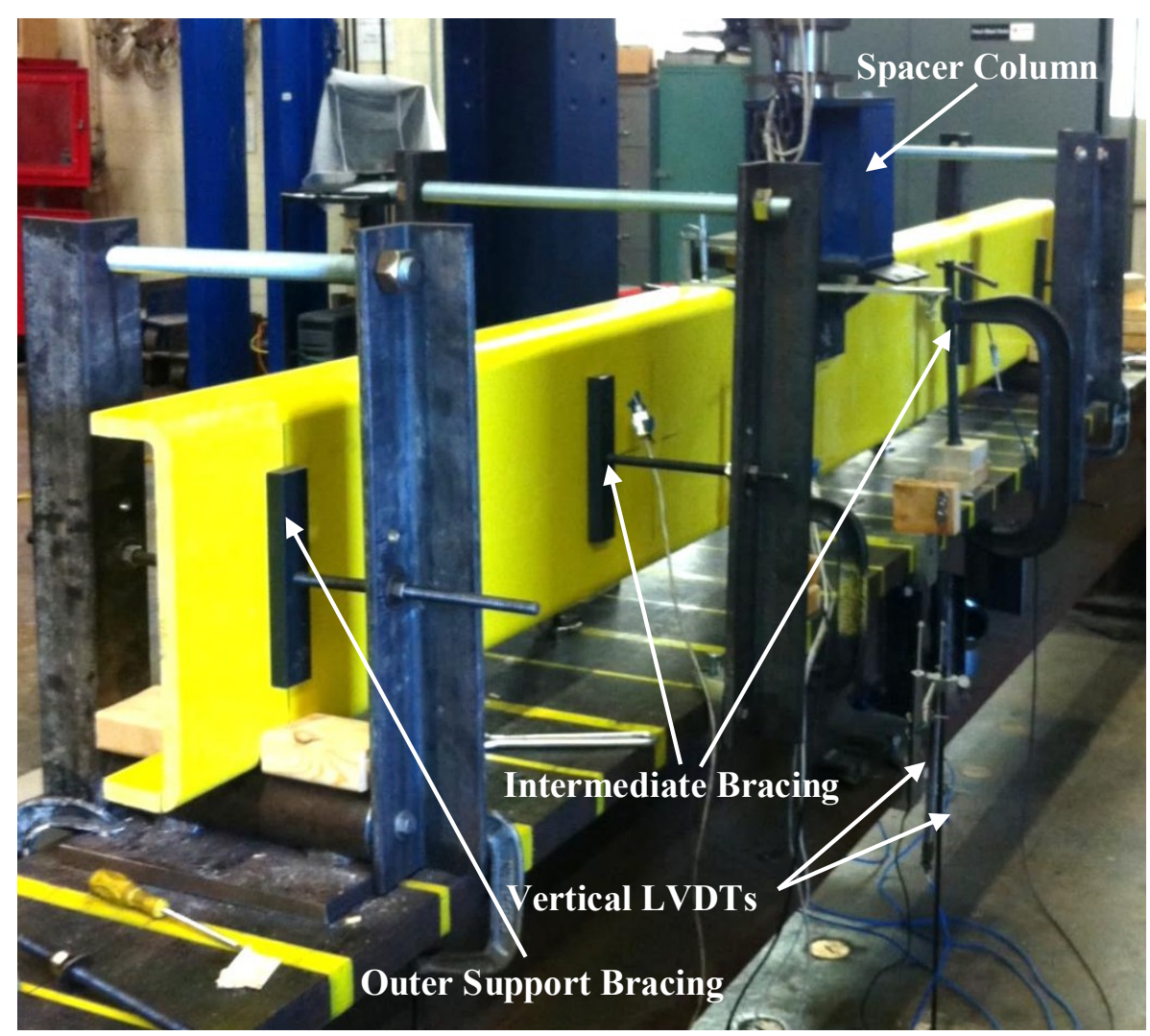

Figure 4-5 Rear view of test setup for 14 " $\times 3.5 " \times 0.75$ " channel section subjected to three-point bending with intermediate lateral brace points $2.5 \mathrm{ft}$. from midspan

\subsection{Experimental Data Analysis}

The analysis of the experimental data generated by the author from over 100 tests performed on a number of channel and WF sections led to the identification of a critical LTB load range defined by lower and upper bounds for each test specimen. The decision to classify the critical LTB load by a range, rather than one specific load level, was a result of some variations in plotted data throughout the test matrix. The lower and upper bounds were selected based on slope changes observed in plots of vertical applied load versus 45 degree strain, and verified by plots of vertical applied load versus horizontal deflection, generated using collected experimental data. By defining these bounds based on test data where the setup consisted of no intermediate bracing, the critical LTB load is captured. It should be noted that each of the 
specimens tested by the author underwent a number of replications in which lateral bracing was applied using different techniques, and at different locations. The subsequent analysis revealed that although overall lateral movement was reduced, the intermediate lateral bracing techniques used in this study did not drastically increase the critical LTB load. Development of the prediction model intended to accurately predict the upper bound of this critical LTB load range. This process safeguards against over-prediction of the critical LTB load.

Development of the critical LTB load range is performed strictly based on visual inspection of the aforementioned plots. Plots of strain measured at 45 degrees at mid-depth of the web will be linear leading up to the point at which buckling initiates. By tracing the initial linear portion of the plot, an initial minor change in slope can be identified - this point serves as the lower bound of the critical LTB load range. Then, by tracing the linearity of the portion of the plot just beyond the lower bound, a second major change in slope can be pinpointed - this point serves as the upper bound of the critical LTB load range. A distinct percent change in slope which would indicate the exact location of the lower and upper bounds of the critical LTB load range has not been quantified. Two examples of this analysis process is demonstrated in Figure 4-6 for (a) a test of a $5.5 " \times 5.5 " \times 0.25$ " channel specimen at a span of 108 inches and (b) a test of $8 " \times 8 " \times 0.375$ " WF specimen at a span of 108 inches. Any variation in experimental data which deviated from the typical 45 degree strain plot led to visual verification using the load versus horizontal deflection plot. Using this plot, the initiation of deformation in the web can be identified and used to confirm the critical LTB load range. Additional experimental vertical applied load versus 45 degree strain and vertical applied load versus horizontal deflection plots can be found in Appendix D. Plots shown in this Appendix exemplify some of aforementioned variations in plotted data which led to the development of the critical LTB load range. 


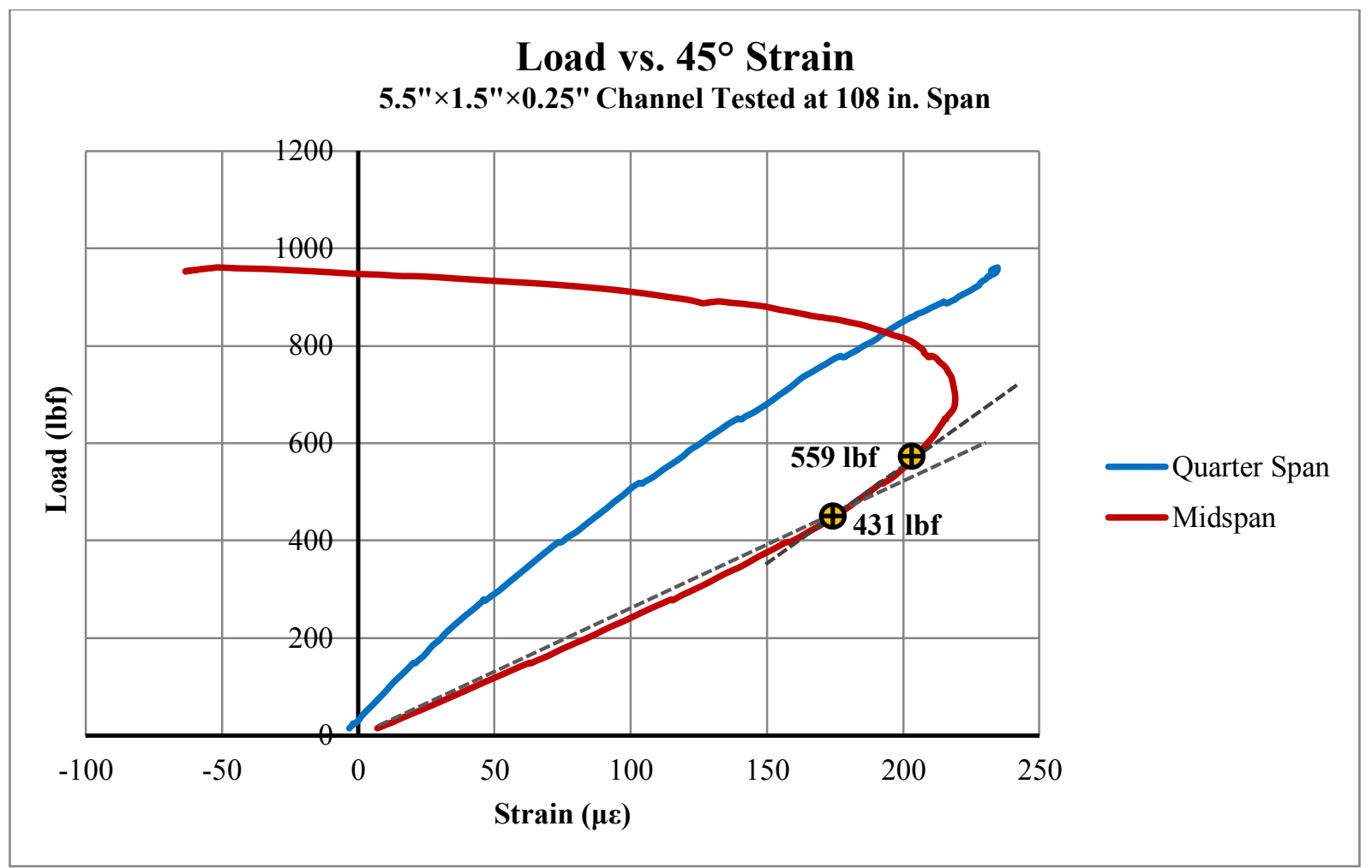

(a)

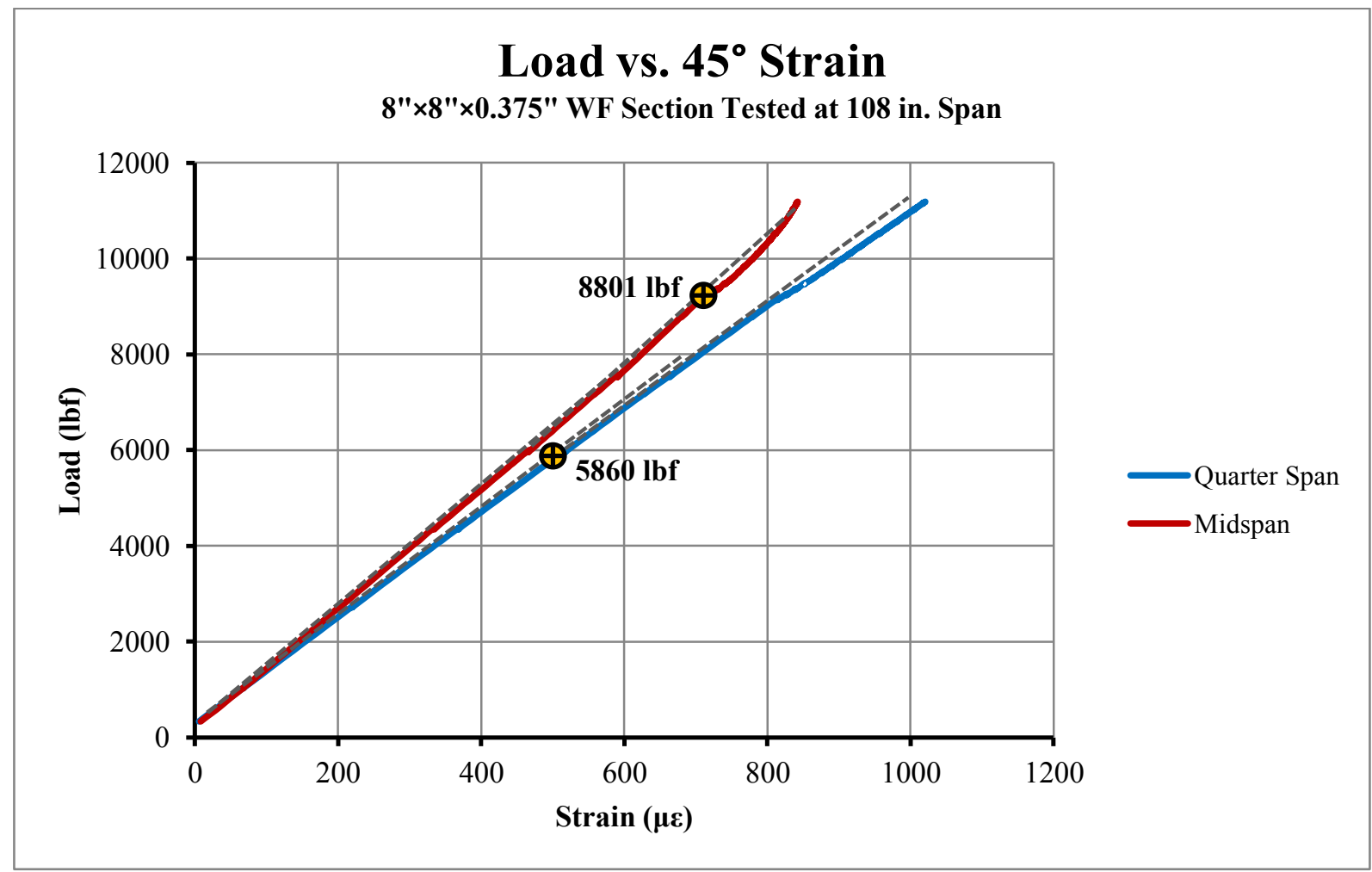

(b)

Figure 4-6 (a) and (b) Selection of lower and upper bounds of critical LTB load range by visual inspection 


\subsection{Critical LTB Load Prediction Model}

An equation for the elastic lateral-torsional buckling strength can be derived from the moment curvature relationship for beams subjected to transverse loads, as shown in Equations (4-1) and (4-2), as well as the torsional differential equation shown in Equation (4-3) (Salmon and Johnson 1996)

$$
\begin{gathered}
E I_{x} \frac{d^{2} v}{d z^{2}}=M_{0} \\
E I_{y} \frac{d^{2} u}{d z^{2}}=M_{0} \varphi \\
-\frac{d u}{d z} M_{0}=G J \frac{d \varphi}{d z}-E C_{w} \frac{d^{3} \varphi}{d z^{3}}
\end{gathered}
$$

In which

$E=$ modulus of elasticity (psi)

$I_{x}=$ strong-axis moment of inertia (in. $\left.{ }^{4}\right)$

$I_{y}=$ weak-axis moment of inertia (in. $\left.{ }^{4}\right)$

$u=$ displacement of centroid in $\mathrm{x}$-direction (in.)

$v=$ displacement of centroid in $\mathrm{y}$-direction (in.)

$M_{0}=$ applied moment (lbf·in.)

$G=$ shear modulus (psi)

$J=$ torsion constant $\left(\right.$ in. $\left.^{4}\right)$

$C_{w}=$ torsional warping constant (in.6)

$\varphi=$ angle of twist (radians)

The $x y z$ coordinate system is shown in Figure 4-1. Two assumptions that led to the development of Equations (4-1) and (4-2) related to small deformations are: (1) the moments of 
inertia, $I_{x}$ and $I_{y}$, remain unchanged after the section has begun to move out-of-plane, and (2) the strong-axis moment of inertia, $I_{x}$, is much larger than the weak-axis moment of inertia, $I_{y}$. From this derivation, a direct equation for calculating the elastic LTB strength, $M_{c r}$, of an open section subjected to a constant moment in the plane of the web is well established for steel and is shown in Equation (4-4) (Salmon and Johnson 1996)

$$
M_{c r}=C_{b} \frac{\pi}{L} \sqrt{\left(\frac{\pi E}{L}\right)^{2} C_{w} I_{y}+E I_{y} G J}
$$

In which

$C_{b}=$ modification factor for non-uniform bending moment variation for a beam segment laterally unbraced except at the ends of the beam segment

$L=$ laterally unbraced length (in.)

$E=$ compression modulus of elasticity (psi)

$C_{w}=$ torsional warping constant $\left(\right.$ in. $^{6}$ )

$I_{y}=$ weak-axis moment of inertia of one flange (in. ${ }^{4}$ )

$G=$ shear modulus (psi)

$J=$ torsion constant (in. $\left.{ }^{4}\right)$

The compressive $E$ modulus is used here because LTB initiates in the compression zone of the web. In the absence of compressive E modulus data, the characteristic longitudinal modulus of the web, $E_{L, w}$, based on coupon tension testing is used for calculations in this study. The resulting error should be minimal because the compressive and tensile $E$ moduli are nearly equal at low strain levels, which are characteristic of LTB failures. It should be noted here that the modification factor, $C_{b}$, can be conservatively taken to be equal to one for GFRP sections. This is because GFRP sections are much more flexible than steel counterparts, and therefore the 
effect of triangular moment induced under three-point bending is not providing a substantial increase to the critical moment compared to a uniform bending moment application. The critical LTB moment can then be converted to a critical LTB load based on the fundamental moment equation for three-point bending with the concentrated load at midspan, shown in Equation (4-5)

$$
M=\frac{P L}{4}
$$

In which

$P=$ applied load (lbf)

$L=$ total span length (in.)

By substituting Equation (4-5) into Equation (4-4), Equation (4-6) is obtained which may be used to calculate the predicted critical LTB load due to concentrated load acting at the midspan

$$
P_{c r}=C_{b} \frac{4 \pi}{L^{2}} \sqrt{\left(\frac{\pi E}{L}\right)^{2} C_{w} I_{y}+E I_{y} G J}
$$

In which

$P_{c r}=$ predicted critical LTB load (lbf)

$C_{b}=$ modification factor for non-uniform bending moment variation for a beam segment laterally unbraced except at the ends of the beam segment

$L=$ laterally unbraced length (in.)

$E=$ compression modulus of elasticity (psi)

$C_{w}=$ torsional warping constant (in. ${ }^{6}$ )

$I_{y}=$ weak-axis moment of inertia of one flange (in. ${ }^{4}$ )

$G=$ shear modulus (psi)

$J=$ torsion constant (in. $\left.{ }^{4}\right)$ 
The properties $I_{y}, J$, and $C_{w}$ are dependent on the dimensions and type of the specimen cross section, as discussed in Sections 4.4.1 and 4.4.2.

\subsubsection{WF Sections}

Accurate prediction of the critical buckling load for pultruded GFRP wide flange sections is partially dependent on effective flange width $\left(b_{\text {eff }}\right)$. Longitudinal stresses are distributed across the compression flange. Due to the influence of shear deformation, plane sections do not remain plane, and therefore the longitudinal stress distribution in the compression flange is non-uniform. Longitudinal stresses in the compression flange decrease as the distance from the web increases due to the shear lag phenomenon. The effective flange width represents the portion of the compression flange over which the member moments resulting from the assumed uniform longitudinal stress distribution calculated from elementary beam theory are equal to those produced by the experimental non-uniform stress distribution (AASHTO 2013). A direct equation for calculating the effective flange width in a pultruded wide flange section was presented by Lopez-Anido and GangaRao (1996), as shown in Equation (4-7)

$$
b_{e f f} \cong \frac{b_{f}}{k_{f}^{2}+1}
$$

where

$$
k_{f}=\frac{b_{f}}{L} \sqrt{\frac{E_{L, f}}{G_{L T, f}}}
$$

In which

$k_{f}=$ shear lag effect factor (equal to zero for no shear lag effects)

$b_{f}=$ full width of the flange (in.)

$L=$ total span length (in.) 
$E_{L, f}=$ characteristic longitudinal modulus of the flange (psi)

$G_{L T, f}=$ characteristic value of the in-plane shear modulus of the flange (psi)

For wide flange GFRP sections, the compression flange behaves elastically, and bends about the weak axis due to the low transverse modulus value characteristic of pultruded sections. Therefore, the flange is assumed to be two separate half-flanges on either side of the web acting independently of one another, especially as fiber continuity between web and flange (at the junction) is typically non-existent. The bending axis of the half-flange is not about its center, nor is it about the end of the half-flange; rather, the bending axis occurs somewhere between the web-flange junction and the center of the half-flange due to lack of 100 percent rigidity between the flange and the web. This theory has been demonstrated through experimental data collected by utilizing a series of strain gages on the underside of the compression flange during testing. This phenomenon is exemplified by the plot in Figure 4-7 which shows the longitudinal strain on the underside of the compression flange of an 8 in. $x 8$ in. $x 0.375$ in. section at locations 1,2 , and 3 inches from the web. It should be noted that negative strain values indicate compression. Visual inspection during testing also suggests that the significant fillet at the reentrant webflange corners contributes added stiffness due to the high resin-volume fraction at these locations. 


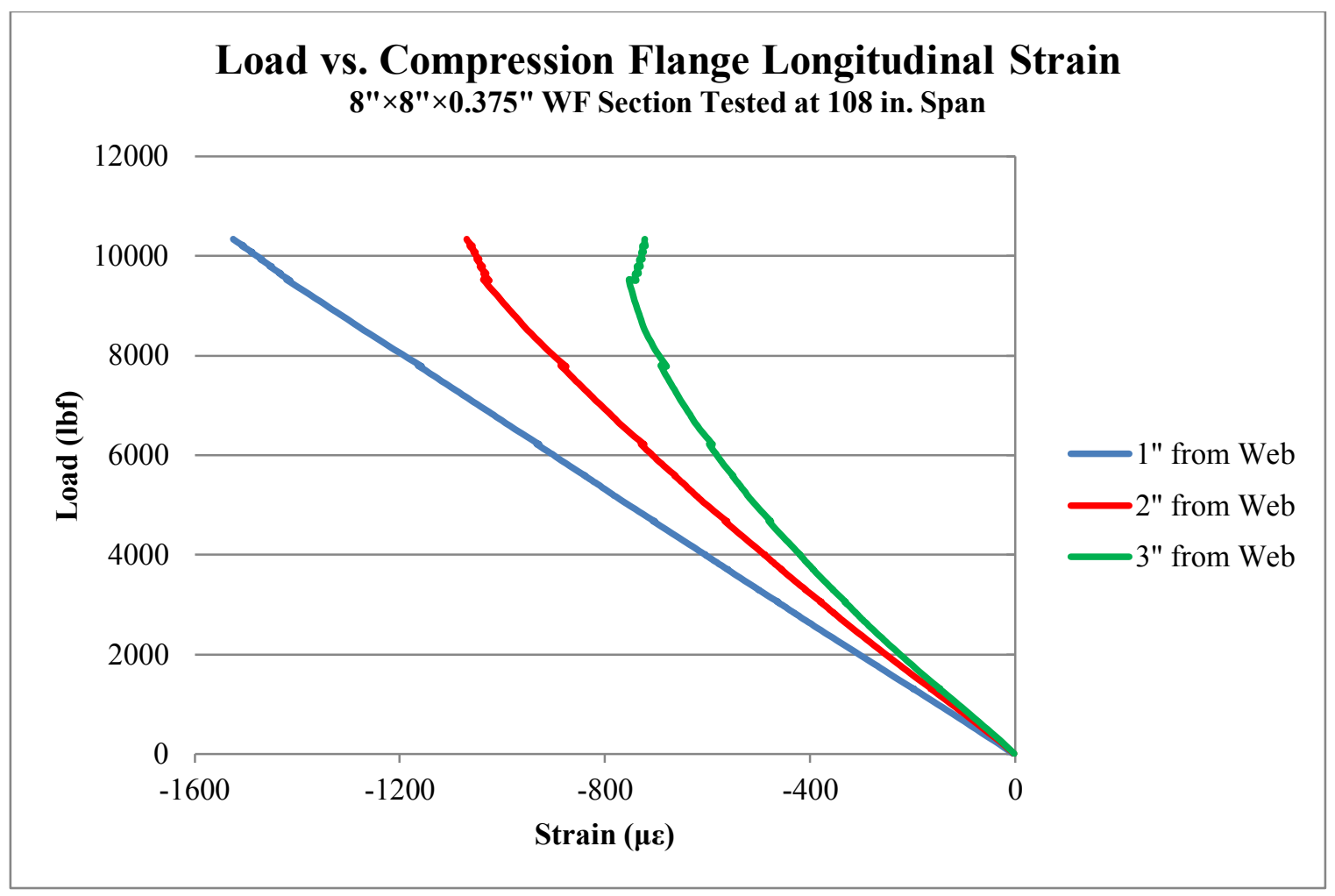

Figure 4-7 Load vs. longitudinal strain in the compression flange at varying distances from the web for a $8^{\prime \prime} \times 8^{\prime \prime} \times 0.375 "$ WF section tested at a span of $108 \mathrm{in}$.

This bending of the half-flanges is related to the torsional response of the compression flange under bending and related to the torsional warping constant, $C w$, and the torsion constant, $J$, for wide flange sections. Conversely, the weak axis moment of inertia of one flange, $I_{y}$, used to apply Equation (4-6) is related to the global bending resistance of the compression flange. Therefore, the equation for the weak axis moment of inertia, to account for web stiffness based on experimental observations, is postulated to be

$$
I_{y}=\frac{1}{6} t_{f} b_{e f f}^{3}
$$

In which

$t_{f}=$ flange thickness (in.)

$b_{\text {eff }}=$ effective flange width (in.), as calculated in Equation (4-7) 
The postulated value of web stiffness is twice that of the conventional value, which is justified because of the additional stiffness offered by the fillet at the web-flange junction. Equations for calculating the torsional warping constant and the torsion constant of wide flange sections are shown in Equations (4-10) and (4-11)

$$
\begin{gathered}
C_{\omega}=\frac{1}{12} t_{f}\left(\frac{b_{\text {eff }}}{2}\right)^{3} \times \frac{d_{w}{ }^{2}}{2} \times 2 \\
J=2 \times \frac{1}{3} b_{f} t_{f}{ }^{3}+\frac{1}{3} t_{w}{ }^{3} d_{w}
\end{gathered}
$$

In which

$t_{f}=$ thickness of the flange (in.)

$b_{\text {eff }}=$ effective flange width (in.), as calculated in Equation (4-7)

$d_{w}=$ depth of the web (in.)

$b_{f}=$ full width of the flange (in.)

$t_{w}=$ thickness of the web (in.)

It should be noted that Equations (4-10) and (4-11) include a factor of 2 due to the two half-flanges undergoing torsional response. It should also be noted that the effective flange width pertains to resistance of the bending moment and not to torsional resistance; therefore, the full width of the flange $\left(b_{f}\right)$ is used when calculating the torsion constant, $J$, as shown in Equation (4-11).

\subsubsection{Channel Sections}

Bending behavior is significantly different for channel specimens. This is because the cross-section is not symmetric about the major bending axis, making the section more susceptible to movement out-of-plane during bending when the transverse load is away from the 
shear center. This issue of twisting greatly reduces the overall capacity of the section. Because shear center loading is unrealistic in most field applications, consistently accurate prediction of the critical LTB load based on loading at the center of gravity is of utmost importance in designing with these types of sections. This explains why channels are rarely used as beams, except where two channel sections are used back-to-back; rather, these sections are used most often as both end and intermediate lateral bracing members. Similarly, LTB causes a fraction of the bending moment to be transferred to the weak axis, which is absorbed mostly by the flanges. Therefore, contribution of the web can be neglected in calculating the weak-axis moment of inertia as will be shown.

Accurate prediction of the critical LTB load for GFRP channels is dependent on the ratio of the thickness of the compression flange to the average radius, $r$, of the reentrant corner $\left(t_{f} / r\right)$, which was determined to be very influential in determining the significance of the added stiffness of the web-flange junction of these sections. The effect of stress concentration at the fillet is much larger in more flexible (smaller) sections than that of more stiff (larger) cross sections due to larger deformations under the applied load. Thus, the presence of stress concentration at the fillet cannot be neglected when discussing lateral-torsional buckling, especially in the case of GFRP sections. A stress concentration for a rounded fillet at a corner of a rectangular tubular steel member subjected to torsion was developed by Trefftz (1922). It should be noted that this corner stress concentration factor increases as the average radius of the corner decreases, therefore typically resulting in a larger stress concentration factor for smaller, more flexible sections which is consistent with the theory presented herein. The corner stress concentration factor, $k$, as derived by Trefftz (1922), is calculated according to Equation (4-12) 


$$
k=\beta \sqrt[3]{\frac{t_{f}}{r}}
$$

where $\beta$ is a constant dependent on the torsional stiffness of the compression flange, $J_{f}$, which can be calculated as shown in Equation (4-13)

$$
J_{f}=\frac{1}{3} b_{f} t_{f}^{3}
$$

In which

$b_{f}=$ full width of the flange (in.)

$t_{f}=$ thickness of the flange (in.)

The constant, $\beta$, was originally defined for closed steel sections. Preliminary results obtained through empirical analysis show that when $J_{f}$ is greater than or equal to $0.01, \beta$ is equal to 1 . Similarly, this analysis also shows that when $J_{f}$ is less than $0.01, \beta$ is equal to 2 . This constant has to be modified for different values of $J_{f}$ to provide good accuracy for open sections. The corner stress concentration factor, $k$, is then applied to the flange thickness in calculating the torsional warping constant, $C_{w}$, the torsion constant, $J$, and the weak-axis moment of inertia of one flange, $I_{y}$, as shown in Equations (4-14) through (4-16).

$$
\begin{gathered}
C_{w}=\frac{1}{3} k t_{f}\left(b_{f}-\frac{t_{w}}{2}\right)^{3} \times \frac{\left(h-k t_{f}\right)^{2}}{2} \\
J=2 \times \frac{1}{3}\left(b_{f}-\frac{t_{w}}{2}\right)\left(k t_{f}\right)^{3}+\frac{1}{3} t_{w}^{3} d_{w} \\
I_{y}=2 \times \frac{1}{3} k t_{f}\left(b_{f}-\frac{t_{w}}{2}\right)^{3}
\end{gathered}
$$


In which

$k=$ corner stress concentration factor

$t_{f}=$ thickness of the flange (in.)

$b_{f}=$ full width of the flange (in.)

$t_{w}=$ thickness of the web (in.)

$h=$ total height of the section (in.)

$d_{w}=$ depth of the web (in.)

It should be noted that the concept of effective flange width does not need to be applied for channel sections. This is because for channel specimens similar to those presented within this report tend to have very short flanges, therefore $b_{\text {eff }} \cong b_{f}$, as shown in Table 4-5. It is possible that for larger channel specimens having longer flanges the influence of the effective flange width could be significant, but the effect is considered as negligible for this study.

Table 4-5 Comparison of $b_{f}$ and $b_{e f f}$ for channel specimens

\begin{tabular}{|l|c|c|c|}
\multicolumn{1}{|c|}{ Sample } & b $_{\text {f }}$ (in.) & beff $_{\text {(in) }}$ & \% Change \\
\hline $\mathbf{1 4} " \times \mathbf{3 . 5} " \times \mathbf{0 . 7 5 "}$ & 3.5 & 3.462767 & $-1.06 \%$ \\
\hline $\mathbf{1 0 "} \times \mathbf{2 . 7 5 "} \times \mathbf{0 . 5}$ & 2.75 & 2.731866 & $-0.66 \%$ \\
\hline $\mathbf{8}^{\prime \prime} \times \mathbf{2 . 2 5} " \times \mathbf{0 . 3 7 5 "}$ & 2.25 & 2.24 & $-0.44 \%$ \\
\hline $\mathbf{6}^{\prime \prime} \times \mathbf{1 . 6 2 5} " \times \mathbf{0 . 1 8 7 5 "}$ & 1.625 & 1.621225 & $-0.23 \%$ \\
\hline $\mathbf{5 . 5} \times \mathbf{1 . 5}^{\prime \prime} \times \mathbf{0 . 2 5 "}$ & 1.5 & 1.497043 & $-0.20 \%$ \\
\hline $\mathbf{4}^{\prime \prime} \times \mathbf{1 . 1 2 5} \times \mathbf{0 . 1 8 7 5 "}$ & 1.125 & 1.123746 & $-0.11 \%$ \\
\hline
\end{tabular}

\subsection{Existing LTB Prediction Models}

Two other existing equations for the prediction of the critical LTB load were considered in this study. The first equation is from the most recently proposed draft version of the ASCE/ACMA Pre-Standard for Load and Resistance Factor Design (LRFD) of Pultruded Fiber 
Reinforced Polymer (FRP) Structures, as modified by Dan Witcher. This version of the critical LTB moment, shown in Equation (4-17), is not given in the most recent publicly available version of the Pre-Standard, but is currently in consideration for inclusion in the LRFD design model.

$$
M_{c r}=C_{b} \sqrt{\frac{\pi^{2} E_{L, f} I_{y} D_{J}}{L_{b}^{2}}+\frac{\pi^{4} E_{L, f}^{2} I_{y} C_{w}}{L_{b}^{4}}}
$$

In which

$M_{c r}=$ elastic lateral-torsional buckling strength (lbf $\cdot$ in)

$C_{b}=$ modification factor for non-uniform bending moment variation for a beam segment laterally unbraced except at the ends of the beam segment

$E_{L, f}=$ characteristic longitudinal modulus of the flange (psi)

$I_{y}=$ weak-axis moment of inertia of one flange (in. ${ }^{4}$ )

$D_{J}=$ torsional rigidity $\left(\mathrm{lbf} \cdot \mathrm{in}^{2}{ }^{2}\right)$, calculated as shown in Equation (4-18) (Timoshenko 1961)

$L_{b}=$ distance between lateral bracing points (in.)

$C_{w}=$ torsional warping constant $\left(\right.$ in. $\left.{ }^{6}\right)$, calculated as shown in Equation (4-19)

$$
\begin{gathered}
D_{J}=G_{L T} \sum_{i} \frac{1}{3} b_{f} t_{f}^{3} \\
C_{w}=\frac{t_{f} h^{2} b_{f}^{3}}{24}
\end{gathered}
$$

In which

$G_{L T}=$ characteristic value of the in-plane shear modulus (psi)

$b_{f}=$ full width of the flange (in.)

$t_{f}=$ thickness of the flange (in.) 
$h=$ full height of the section (in.)

The nominal moment can be converted to a critical LTB load by substituting Equation (4-5) into Equation (4-17), as shown in Equation (4-20)

$$
P_{c r}=\frac{4 C_{b}}{L} \sqrt{\frac{\pi^{2} E_{L, f} I_{y} D_{J}}{L_{b}^{2}}+\frac{\pi^{4} E_{L, f}^{2} I_{y} C_{w}}{L_{b}^{4}}}
$$

In which

$P_{c r}=$ predicted critical LTB load (lbf)

$C_{b}=$ modification factor for non-uniform bending moment variation for a beam segment laterally unbraced except at the ends of the beam segment

$L=$ laterally unbraced length (in.)

$E_{L, f}=$ characteristic longitudinal modulus of the flange (psi)

$I_{y}=$ weak-axis moment of inertia $\left(\right.$ in. $\left.^{4}\right)$

$D_{J}=$ torsional rigidity $\left(\mathrm{lbf} \cdot \mathrm{in}^{2}{ }^{2}\right)$, calculated as shown in Equation (4-18) (Timoshenko 1961)

$L_{b}=$ distance between lateral bracing points (in.)

$C_{w}=$ torsional warping constant (in. ${ }^{6}$ ), calculated as shown in Equation (4-19)

A major issue with Equation (4-17) is that it is derived for doubly-symmetric cross sections, and is therefore not applicable to channel sections.

The second equation considered comes from the Structural Plastics Design Manual (ASCE 1984), which was developed for composites, shown in Equation (4-21).

$$
\sigma_{x c}=\frac{C_{1}}{S_{1}} \sqrt{M_{x c}^{2}+\frac{h^{2}}{4} P_{e 2}^{2}}
$$


In which

$\sigma_{x c}=$ critical LTB stress (psi)

$C_{l}=$ lateral buckling coefficient, taken to be 1.35 for a triangular moment

$S_{l}=$ strong-axis section modulus (in. $\left.{ }^{3}\right)$

$M_{x c}=$ critical buckling moment (lbf $\cdot$ in.), as calculated in Equation (4-22)

$h=$ full height of the section (in.)

$P_{e 2}=$ Euler column load for buckling in the weak direction (lbf), as calculated in Equation (4-23)

$$
\begin{gathered}
M_{x c}=\frac{\pi}{K L_{b}} \sqrt{E I_{y} G J} \\
P_{e 2}=\frac{\pi^{2} E I_{y}}{\left(K L_{b}\right)^{2}}
\end{gathered}
$$

Where

$\mathrm{K}=$ effective length factor, taken as equal to 1 for the case of a simply supported beam

$L_{b}=$ distance between lateral bracing points (in.)

$E=$ compression modulus of elasticity (psi)

$I_{y}=$ weak-axis moment of inertia (in. $\left.{ }^{4}\right)$

$G=$ shear modulus (psi)

$J=$ torsion constant (in. ${ }^{4}$ )

The critical buckling flexural stress can be converted to the critical LTB load by combining Equations (4-5) and (4-24), which is the classic flexure formula where $c=$ distance from neutral axis to the outside tension or compression fiber of the beam (in.) and $I_{x}=$ strongaxis moment of inertia, and substituting into Equation (4-21). By making these substitutions, Equation (4-25) is obtained. 


$$
\begin{gathered}
\sigma=\frac{M c}{I} \\
P_{c r}=\frac{4 I_{x}}{L c} \frac{C_{1}}{S_{1}} \sqrt{M_{x c}^{2}+\frac{h^{2}}{4} P_{e 2}^{2}}
\end{gathered}
$$

In which

$I_{x}=$ strong-axis moment of inertia (in. $\left.{ }^{4}\right)$

$L=$ laterally unbraced length (in.)

$c=$ distance from neutral axis to the outside tension or compression fiber of the beam (in.) $=h / 2$

$C_{l}=$ lateral buckling coefficient, taken to be 1.35 for a triangular moment

$S_{I}=$ strong-axis section modulus (in. $\left.{ }^{3}\right)$

$M_{x c}=$ critical buckling moment (lbf $\cdot$ in.), as calculated in Equation (4-22)

$h=$ full height of the section (in.)

$P_{e 2}=$ Euler column load for buckling in the weak direction (lbf), as calculated in Equation (4-23)

Equation (4-21) assumes the beams to be doubly-symmetric with load applied at the shear center, and thus is also not applicable for channel sections. Results of the existing prediction models compared to the experimental results of this study can be found in Section 4.7.

\subsection{Results of Proposed LTB Prediction Model}

The results from the model proposed by the author using Equation (4-6) with modifications to section property calculations as detailed in Sections 4.4.1 and 4.4.2 are discussed herein in the following two sections. 


\subsubsection{WF Sections}

Consistent and accurate prediction of the critical LTB load is achieved when the effective flange width is taken into consideration when applying the proposed model for WF sections. Using Equation (4-6), error in critical buckling load prediction fell within $\pm 15 \%$ error of the experimental upper bound of the critical LTB load range for all specimens. In addition to those samples tested by the author, a 12 " $\times 12$ " $\times 0.5$ " section tested under four-point bending at a span length of 180 inches by Bendidi (1996) was also included in the analysis. For this section, critical lateral-torsional buckling moment must be converted to a critical LTB load using Equation (4-26), which represents the maximum moment for a beam subjected to four-point bending with the applied loads located a distance $L / 3$ from the outer supports.

$$
M_{c r}=\frac{P_{c r} L}{6}
$$

In which

$M_{c r}=$ elastic lateral-torsional buckling strength (lbf·in), as calculated in Equation (4-4)

$P_{c r}=$ predicted critical LTB load (lbf)

$L=$ total span length (in.)

As can be seen in Table 4-6, the proposed model provides consistent accuracy in predicting the critical LTB load based on the average of multiple tests of four WF sections performed by Estep. The model also proves applicable to the 12 " $\times 12$ " $\times 0.5$ " WF specimen tested under four-point bending by appropriately modifying the equation for four-point bending loads. Using Equation (4-6), with modifications as discussed in Section 4.4.1, prediction of the critical LTB load fell within \pm 13.7 percent of the experimental upper bound of the critical LTB load 
range for all WF sections. The mean error for prediction of the upper bound of the critical LTB load range for WF sections based on this equation is +3.7 percent.

Table 4-6 Critical LTB load prediction for WF sections using the proposed model

\begin{tabular}{|c|c|c|c|c|c|c|c|c|c|}
\hline \multirow[b]{2}{*}{ Sample } & \multirow[b]{2}{*}{$\mathbf{I}_{\mathbf{y}}\left(\mathrm{in}^{4}\right)$} & \multirow[b]{2}{*}{$C_{w}\left(i^{6}\right)$} & \multirow[b]{2}{*}{$\mathbf{J}\left(\right.$ in $\left.^{4}\right)$} & \multicolumn{2}{|c|}{ Predicted Values } & \multicolumn{2}{|c|}{$\begin{array}{c}\text { Experimental } \\
\text { Data }\end{array}$} & \multicolumn{2}{|c|}{ Percent Error } \\
\hline & & & & $\begin{array}{c}\mathbf{M}_{\mathrm{cr}} \\
(\text { lbf } \cdot \text { in })\end{array}$ & $\mathbf{P}_{\mathrm{cr}}(\mathrm{lbf})$ & $\begin{array}{l}\text { LB } \\
(\mathrm{lbf})\end{array}$ & $\begin{array}{l}\text { UB } \\
\text { (lbf) }\end{array}$ & $\begin{array}{c}\% \text { Error } \\
\text { LB }\end{array}$ & $\begin{array}{c}\text { \% Error } \\
\text { UB }\end{array}$ \\
\hline $12 " \times 12 " \times 0.5 " *$ & 133.83 & 1012.08 & 1.46 & 645864 & 21529 & 15174 & 19334 & $41.9 \%$ & $11.4 \%$ \\
\hline $10^{\prime \prime} \times 5 " \times 0.5 "$ & 9.76 & 49.39 & 0.792 & 159399 & 5904 & 4541 & 5242 & $28.7 \%$ & $12.6 \%$ \\
\hline $8 " \times 8 " \times 0.375^{\prime \prime}$ & 27.14 & 89.16 & 0.409 & 262060 & 9706 & 5860 & 8801 & $63.8 \%$ & $10.3 \%$ \\
\hline $8^{\prime \prime} \times 4^{\prime \prime} \times 0.375^{\prime \prime}$ & 3.64 & 11.96 & 0.268 & 93726 & 5207 & 3520 & 6032 & $46.4 \%$ & $-13.7 \%$ \\
\hline $6 " \times 6 " \times 0.375 "$ & 12.00 & 20.66 & 0.303 & 131069 & 5461 & 2718 & 5573 & $98.9 \%$ & $-2.0 \%$ \\
\hline
\end{tabular}

*denotes section which was tested under four-point bending by Bendidi (1996)

Note: LB denotes lower bound and UB denotes upper bound of critical lateral-torsional buckling load range.

\subsubsection{Channel Sections}

By applying a modified version of the equation for calculating the corner stress concentration factor, $k$, to the thickness of the flange, as discussed in Section 4.4.2, the proposed critical LTB load prediction model exhibits good consistency and accuracy for all channel specimens tested, as exhibited by the results shown in Table 4-7. Using Equation (4-6), prediction of the critical LTB load fell within \pm 10 percent of the experimental upper bound of the critical LTB load range for nearly all tested channel specimens, with the exception of the $10 " \times 2.75$ " $\times 0.5$ " channel section, which exhibited a percent error of -15.2 percent. The mean error for prediction of the upper bound of the critical LTB load range for channel sections based on this equation is -0.7 percent. 
Table 4-7 Critical LTB load prediction for channel sections using proposed model

\begin{tabular}{|c|c|c|c|c|c|c|c|c|c|}
\hline \multirow[b]{2}{*}{ Sample } & \multirow[b]{2}{*}{$\mathbf{I}_{\mathbf{y}}\left(\mathrm{in}^{4}\right)$} & \multirow[b]{2}{*}{$\mathrm{C}_{\mathrm{w}}\left(\mathrm{in}^{6}\right)$} & \multirow[b]{2}{*}{$\mathbf{J}\left(\mathrm{in}^{4}\right)$} & \multicolumn{2}{|c|}{$\begin{array}{l}\text { Predicted } \\
\text { Values }\end{array}$} & \multicolumn{2}{|c|}{$\begin{array}{c}\text { Experimental } \\
\text { Data }\end{array}$} & \multicolumn{2}{|c|}{ Percent Error } \\
\hline & & & & $\begin{array}{c}\mathbf{M}_{\mathrm{cr}} \\
(\text { lbf } \cdot \mathrm{in})\end{array}$ & $\begin{array}{r}\mathbf{P}_{\mathrm{cr}} \\
(\mathrm{lbf})\end{array}$ & $\begin{array}{l}\text { LB } \\
\text { (lbf) }\end{array}$ & $\begin{array}{l}\text { UB } \\
\text { (lbf) }\end{array}$ & $\begin{array}{c}\% \text { Error } \\
\text { LB }\end{array}$ & $\begin{array}{l}\text { \% Error } \\
\text { UB }\end{array}$ \\
\hline $14 " \times 3.5 " \times 0.75 "$ & 16.21 & 706.64 & 2.81 & 470511 & 17426 & 14022 & 16050 & $24.3 \%$ & $8.6 \%$ \\
\hline $10 " \times 2.75 " \times 0.5 "$ & 5.21 & 117.51 & 0.583 & 112896 & 4181 & 3735 & 4929 & $11.9 \%$ & $-15.2 \%$ \\
\hline $8 " \times 2.25 " \times 0.375^{\prime \prime}$ & 2.24 & 32.55 & 0.205 & 48461 & 1795 & 1168 & 1870 & $51.8 \%$ & $-4.0 \%$ \\
\hline $6 " \times 1.625 " \times 0.1875 "$ & 0.837 & 6.68 & 0.056 & 14299 & 530 & 351 & 506 & $49.1 \%$ & $4.7 \%$ \\
\hline $5.5^{\prime \prime} \times 1.5 " \times 0.25 "$ & 0.867 & 5.42 & 0.141 & 15759 & 584 & 431 & 559 & $35.4 \%$ & $4.4 \%$ \\
\hline $4 " \times 1.125^{\prime \prime} \times 0.1875 "$ & 0.291 & 0.945 & 0.051 & 6083 & 225 & 131 & 232 & $70.4 \%$ & $-2.9 \%$ \\
\hline
\end{tabular}

Note: LB denotes lower bound and UB denotes upper bound of critical lateral-torsional buckling load range.

\subsection{Results of Existing LTB Prediction Models}

The results from the existing critical LTB load prediction models analyzed by the author for comparison, as discussed in Section 4.5, are discussed herein in the following two sections.

\subsubsection{ASCE/ACMA LRFD Draft Pre-Standard Equation Results}

Results from the draft ASCE/ACMA Pre-Standard for Load and Resistance Factor Design (LRFD) of Pultruded Fiber Reinforced Polymer (FRP) Structures equation with modifications by Dan Witcher, shown in Equation (4-20), proved to be inconsistent for WF section critical LTB load prediction, showing good accuracy for some sections, while highly inaccurate for others, as shown in Table 4-8. The model proved to be more consistent, but still inaccurate, in predicting the critical LTB load for channel sections, as shown in Table 4-9, and overall was not as accurate as the model proposed by the author. It should be noted that by applying the modified equations for $I_{y}, C_{w}$, and $J$ for both channel and WF sections, as used in the model proposed by the author, to Equation (4-20), slightly better accuracy is obtained, but results remain inconsistent. 
Table 4-8 Critical LTB load prediction for WF sections using the ASCE/ACMA equation

\begin{tabular}{|c|c|c|c|c|c|c|}
\hline & \multicolumn{2}{|c|}{ Predicted Values } & \multicolumn{2}{|c|}{ Experimental Data } & \multicolumn{2}{|c|}{ Percent Error } \\
\hline Sample & $\mathbf{M}_{\mathbf{n}}(\mathrm{lbf} \cdot \mathrm{in})$ & $\mathbf{P}_{\mathrm{cr}}(\mathrm{lbf})$ & LB (lbf) & UB (lbf) & $\begin{array}{c}\% \text { Error } \\
\text { LB }\end{array}$ & $\begin{array}{l}\% \text { Error } \\
\text { UB }\end{array}$ \\
\hline $12 " \times 12 " \times 0.5 " *$ & 1129068 & 37636 & 15174 & 19334 & $148.0 \%$ & $94.7 \%$ \\
\hline $10^{\prime \prime} \times 5 " \times 0.5^{\prime \prime}$ & 249628 & 9245 & 4541 & 5242 & $103.6 \%$ & $76.4 \%$ \\
\hline $8^{\prime \prime} \times 8 " \times 0.375^{\prime \prime}$ & 546190 & 20229 & 5860 & 8801 & $245.2 \%$ & $129.9 \%$ \\
\hline $8^{\prime \prime} \times 4^{\prime \prime} \times 0.375^{\prime \prime}$ & 163294 & 9072 & 3520 & 6032 & $157.7 \%$ & $50.4 \%$ \\
\hline $6 " \times 6 " \times 0.375 "$ & 230032 & 9585 & 2718 & 5573 & $252.6 \%$ & $72.0 \%$ \\
\hline
\end{tabular}

*denotes section which was tested under four-point bending by Bendidi (1996)

Table 4-9 Critical LTB load prediction for channel sections using the ASCE/ACMA equation

\begin{tabular}{|c|c|c|c|c|c|c|}
\hline \multirow[b]{2}{*}{ Sample } & \multicolumn{2}{|c|}{ Predicted Values } & \multicolumn{2}{|c|}{ Experimental Data } & \multicolumn{2}{|c|}{ Percent Error } \\
\hline & $\mathbf{M}_{\mathbf{n}}(\mathrm{lbf} \cdot \mathrm{in})$ & $\mathbf{P}_{\mathrm{cr}}(\mathrm{lbf})$ & LB (lbf) & UB (lbf) & $\begin{array}{c}\% \text { Error } \\
\text { LB }\end{array}$ & $\begin{array}{l}\% \text { Error } \\
\text { UB }\end{array}$ \\
\hline $14 " \times 3.5 " \times 0.75 "$ & 364401 & 13496 & 14022 & 16050 & $-3.7 \%$ & $-15.9 \%$ \\
\hline $10 " \times 2.75 " \times 0.5 "$ & 89485 & 3314 & 3735 & 4929 & $-11.3 \%$ & $-32.8 \%$ \\
\hline $8 " \times 2.25 " \times 0.375 "$ & 36963 & 1369 & 1168 & 1870 & $17.2 \%$ & $-26.8 \%$ \\
\hline $6 " \times 1.625 " \times 0.1875 "$ & 5332 & 197 & 351 & 506 & $-43.7 \%$ & $-61.0 \%$ \\
\hline $5.5^{\prime \prime} \times 1.5 " \times 0.25 "$ & 5472 & 203 & 431 & 559 & $-53.0 \%$ & $-63.7 \%$ \\
\hline $4 " \times 1.125 " \times 0.1875 "$ & 1853 & 69 & 131 & 232 & $-47.6 \%$ & $-70.4 \%$ \\
\hline
\end{tabular}

\subsubsection{Structural Plastics Design Manual Equation Results}

Results from the Structural Plastics Design Manual (ASCE 1984) equation also proved to be inaccurate. For WF sections, this equation was within +225 percent of the experimental upper bound of the critical LTB load range, as shown in Table 4-10. The equation did display some consistency and accuracy in predicting the critical LTB load for larger, more rigid channel specimens, but was inaccurate for smaller, more flexible channels (as shown in Table 4-11) and 
overall did not show the same consistency when compared to the model proposed by the author. It should be noted that by applying the modified equations for $I_{y}, C_{w}$, and $J$ for both channel and WF sections, as used in the model proposed by the author, to Equation (4-25), slightly better accuracy is obtained, but results remain inconsistent.

Table 4-10 Critical LTB load prediction for WF sections using ASCE Plastics Design Manual equation

\begin{tabular}{|c|c|c|c|c|c|c|}
\hline \multirow[b]{2}{*}{ Sample } & \multicolumn{2}{|c|}{ Predicted Values } & \multicolumn{2}{|c|}{ Experimental Data } & \multicolumn{2}{|c|}{ Percent Error } \\
\hline & $\boldsymbol{\sigma}_{\mathrm{xc}}(\mathrm{psi})$ & $\mathbf{P}_{\text {cr }}(\mathrm{lbf})$ & LB (lbf) & UB (lbf) & $\%$ Error LB & $\%$ Error UB \\
\hline $12 " \times 12 " \times 0.5 " *$ & 21563 & 54203 & 15174 & 19334 & $257.2 \%$ & $180.4 \%$ \\
\hline $10^{\prime \prime} \times 5 " \times 0.5^{\prime \prime}$ & 12249 & 13002 & 4541 & 5242 & $186.3 \%$ & $148.0 \%$ \\
\hline $8 " \times 8 " \times 0.375 "$ & 31080 & 28545 & 5860 & 8801 & $387.1 \%$ & $224.3 \%$ \\
\hline $8 " \times 4 " \times 0.375 "$ & 16563 & 12778 & 3520 & 6032 & $263.0 \%$ & $111.8 \%$ \\
\hline $6 " \times 6 " \times 0.375 "$ & 24446 & 13639 & 2718 & 5573 & $401.8 \%$ & $144.7 \%$ \\
\hline
\end{tabular}

*denotes section which was tested under four-point bending by Bendidi (1996)

Table 4-11 Critical LTB load prediction for channel sections using ASCE Plastics Design Manual equation

\begin{tabular}{|c|c|c|c|c|c|c|}
\hline \multirow[b]{2}{*}{ Sample } & \multicolumn{2}{|c|}{ Predicted Values } & \multicolumn{2}{|c|}{ Experimental Data } & \multicolumn{2}{|c|}{ Percent Error } \\
\hline & $\boldsymbol{\sigma}_{\mathbf{x c}}(\mathrm{psi})$ & $\mathbf{P}_{\mathrm{cr}}(\mathrm{lbf})$ & LB (lbf) & UB (lbf) & \% Error LB & $\%$ Error UB \\
\hline $14 " \times 3.5 " \times 0.75 "$ & 10268 & 19164 & 14022 & 16050 & $36.7 \%$ & $19.4 \%$ \\
\hline $10^{\prime \prime} \times 2.75 " \times 0.5 "$ & 6902 & 4728 & 3735 & 4929 & $26.6 \%$ & $-4.1 \%$ \\
\hline $8 " \times 2.25 " \times 0.375 "$ & 5769 & 1947 & 1168 & 1870 & $66.7 \%$ & $4.1 \%$ \\
\hline $6 " \times 1.625 " \times 0.1875 "$ & 2902 & 284 & 351 & 506 & $-19.1 \%$ & $-43.9 \%$ \\
\hline $5.5^{\prime \prime} \times 1.5^{\prime \prime} \times 0.25 "$ & 2698 & 283 & 431 & 559 & $-34.4 \%$ & $-49.4 \%$ \\
\hline $4 " \times 1.125 " \times 0.1875 "$ & 2242 & 95 & 131 & 232 & $-27.8 \%$ & $-59.2 \%$ \\
\hline
\end{tabular}




\subsection{Summary, Conclusions, and Recommendations}

\subsubsection{Determination of Critical LTB Load Range}

The decision to classify the critical LTB load by a range was based on variations in plotted data throughout the test matrix. The lower and upper bounds were selected based on slope changes observed in plots of vertical applied load versus 45 degree strain, and verified by plots of vertical applied load versus horizontal deflection, generated using collected experimental data. At this point, development of the critical LTB load range is performed based on visual inspection as described in Section 4.3, and demonstrated by Figure 4-6 (a) and (b). To improve the accuracy of the experimental evaluation, the critical LTB range should be defined by an exact percent change in slope in either the load versus 45 degree strain plot or load versus horizontal deflection plot, with the lower bound representing a more gradual change in slope than that of the upper bound.

\subsubsection{WF Sections}

The proposed prediction model for WF sections exhibits better accuracy and consistency than existing models for all tested specimens. By modifying the classical elastic LTB equation, the author achieved consistently accurate prediction within \pm 15 percent error of the experimental upper bound of the critical LTB load range for four different sizes of WF sections based on an average taken from a number of three point bending tests. This model also displayed accuracy by predicting within +11.4 percent of the experimental upper bound for the 12 " $\times 12$ " $\times 0.5$ " WF specimen subjected to a four point bend by making the appropriate modification for the maximum moment shown in Equation (4-26). 
Experimental data has shown that the strain in the flange is not uniform and not 100 percent of the peak bending strain, which explains why the effective flange width is used in calculations; however, a more rigorous analysis is required in order to better understand the effect of shear lag and bending of the flanges for accurately representing $C_{w}, I_{y}$, and $J$ of a WF specimen. Furthermore, a variation of the corner stress concentration factor proposed for channel sections may need to be implemented to account for the added stiffness at the web-flange junctions witnessed during experimental observation, and also detected during analysis of data acquired from the testing of WF sections. In design, the values for the transverse and longitudinal elastic moduli, as well as the shear modulus, would be provided for each section size based on catalogued data provided by the manufacturer, allowing for greater accuracy in applying the critical LTB load prediction model. Further refinement of the calculations associated with this model will likely result in improved accuracy in predicting the critical LTB load of wide flange specimens.

\subsubsection{Channel Sections}

The proposed prediction model for channels using Equation (4-20) and applying the corner stress concentration factor, $k$, to the thickness of the flange in calculating $C_{w}, I_{y}$, and $J$, exhibits very good accuracy and consistency. By modifying the classical elastic LTB equation and applying a corner stress concentration factor, $k$, the proposed model displayed accurate prediction of the experimental upper bound of the critical LTB load range for six channel sections based on a number of three point bending tests. Prediction fell within \pm 10 percent of the upper bound for nearly all of the sections tested, with the exception of the 10 " $\times 2.75$ " $\times 0.5$ " channel section which exhibited -15.2 percent error. This section may have damaged during 
previous testing. This data proves the model proposed by the author to be superior to existing models which were originally derived for WF sections.

The influence of the effective flange width is taken to be negligible for channel specimens similar in size to those presented within this report. As shown in Table 4-5, the percent change between $b_{f}$ and $b_{\text {eff }}$ for the specimens presented in this report is less than 1.1 percent. By taking this as negligible, calculation of the critical TLB load is greatly simplified. It is, however, likely that as specimens grow in size (namely, as the channel legs grow in length) the effective flange width will need to be accounted for. At this point, no threshold value has been identified which would determine when $b_{\text {eff }}$ needs to be used in these calculations. Further analysis is also needed to determine the exact relationship between the torsional stiffness of the compression flange, $J_{f}$, and the constant, $\beta$, used in calculating the magnitude of corner stress concentration factor. A more rigorous solution could result in a direct equation for calculating the value of the $\beta$ factor that would consistently match with experimental data.

\subsubsection{Lateral Constraint Mechanisms}

In addition to the development of the critical lateral-torsional buckling load prediction model, the author also did a brief analysis of the effectiveness of different lateral bracing mechanisms. Prior to the initial stages of testing, a mechanism was fabricated allowing for $1 / 2$ inch thick steel bar, cut to about 65 percent of the depth of the web, to be held firmly against the web using threaded rod attached to a fixed steel angle using nuts. This mechanism proved to be effective, especially in the case of more slender sections susceptible to a large amount of horizontal deflection once geometric instability occurs in the compression zone of the web. This mechanism, although not very significant, often resulted in an increase in critical LTB load. 
However, this type of mechanism is difficult to achieve in practice because cross-bracing is placed between two beams which are each susceptible to lateral deformation.

A second mechanism was considered using wooden blocks to brace the outside edges of the flanges by lodging the wood between the sample and a fixed steel angle using wooden construction shims. This method, which is more similar to common practice, proved to be ineffective based on a number of reasons, including the rigidity of the wood being far less than that of the tested sample. This allowed for some deformation in the wooden blocks, therefore resulting in slight movement and insufficient fixity at the lateral bracing positions. Additionally, the mechanism for fixing the wooden blocks into place was ineffective. In a number of tests, the wooden shims fractured, allowing unexpected movement at the lateral bracing positions. This led to irregularities in the slope of vertical applied load versus 45 degree strain plots, and also small jumps in the horizontal deflection plots.

The author believes that a more efficient system for bracing the outer edges of the flanges and web, using fixed steel or FRP lateral constraints, would be an effective bracing mechanism for large, rigid channel specimens. This is based on experimental observation in which stiffer channel specimens had a tendency to roll on the large outer radius of the reentrant corner, allowing the strong-axis for bending of the specimen to move out of plane at the outer supports. 


\section{CHAPTER 5 CONCLUSIONS AND RECOMMENDATIONS}

Chapter five of the ASCE/ACMA Pre-Standard for Load and Resistance Factor Design (LRFD) of Pultruded Fiber Reinforced Polymer (FRP) Structures, for design of members in flexure and shear, has undergone multiple series of revisions since its development began in 2008. When compared with experimental flexural load capacities obtained through extensive testing performed at the West Virginia University Constructed Facilities Center (WVU-CFC), the equations provided in the most recent draft version of chapter five of the Pre-Standard have been shown to be highly inaccurate for both closed and open sections. The objective of this research is to develop a more accurate and easy to compute prediction model for both the critical flexural load capacity of closed GFRP sections, as well as the critical LTB load of open GFRP sections.

This study includes an extensive literature review of previous studies related to prediction of the flexural load capacity of closed and open GFRP sections, as well as hypothetical models related to lateral-torsional buckling based on theories of elastic stability. A series of tests was performed on pultruded GFRP square and rectangular box members, as well as pultruded GFRP

channel and WF sections. Based on the obtained experimental data, as well as data acquired by past WVU-CFC researchers, two prediction models were formulated for determining the critical flexural load capacity of closed GFRP sections and the critical lateral-torsional buckling capacity of GFRP channel and WF sections. Results of these prediction models exhibit good correlation with experimental data over a wide range of specimen sizes and span lengths. 


\subsection{Critical Flexural Capacity of GFRP Box Sections}

Pultruded GFRP square and rectangular box sections of several different sizes were tested under three-point bending to determine the critical flexural capacity at varying test spans. The collected experimental data, as well as data obtained through similar testing by previous WVUCFC researchers, was compared with existing critical flexural load capacity prediction models, and also used in the development of a new prediction model. Detailed results from this testing and analysis can be found in Chapter 3.

Analysis of previous models proposed in draft versions of the ASCE/ACMA Pre-Standard for Load and Resistance Factor Design (LRFD) of Pultruded Fiber Reinforced Polymer (FRP) Structures yielded highly inconsistent and inaccurate results. Thus, a new model for predicting the flexural load capacity of these sections was developed based on experimental observations of failure modes and correlation with test data. This model, presented in Section 3.3, has shown superior accuracy for all section sizes and test spans in comparison to the analyzed existing models. While other failure modes may exist under certain geometric conditions, extensive evaluation of the experimental data revealed that only three primary failure modes control the behavior of pultruded thin-walled GFRP box members in flexure and shear under transverse loading. These three modes of failure are described as: local buckling failure in the compression flange, principal strain failure in the tension zone of the web, and bending failure in the tension flange.

Critical flexural load capacity prediction for local buckling failure in the compression flange yielded results in within $\pm 18.0 \%$ of experimental data for sections tested within the range of $4 \leq L / h<6$. The mean error for flexural capacity prediction within this range of $L / h$ ratios based on experimental data included in this study is $-9.7 \%$. Due to the limited amount of critical 
flexural load capacity data within the range $4 \leq L / h<6$, extensive testing is required to confirm the applicability of the local buckling in the compression flange failure mode.

The prediction equation for principal strain failure in the tension zone of the web predicted the flexural load capacity of thin-walled pultruded GFRP box sections tested in the range $6 \leq L / h \leq 10$ within $\pm 15.5 \%$ of experimental data. The mean error prediction of the flexural load capacity within this range of $L / h$ ratios is $+0.7 \%$ based on experimental data included in this study. For this study, values for the strength and stiffness properties, as well as the mean value of maximum shear strain at failure, of the sections were based on average values from a limited amount of testing. By inserting characteristic values for these properties provided for each section size by the manufacturer, the flexural load capacity can be more accurately predicted.

Long span prediction based on the equation for bending failure in the tension flange has proven to be the most refined component of the prediction model. This equation has been fit to over 50 lab tests, including multiple replications of test specimen sizes and spans within the range of $10<L / h \leq 42$, resulting in a maximum error for prediction of the flexural load capacity of $-22.3 \%$, with over $71 \%$ of all of the data points within $\pm 10 \%$ of the experimental value. The mean error prediction of the flexural load capacity within this range of $L / h$ ratios is $-3.0 \%$.

The accuracy of this prediction model is excellent in comparison to the November 2010 and May 2013 drafts of the ASCE/ACMA Pre-Standard for Load and Resistance Factor Design (LRFD) of Pultruded Fiber Reinforced Polymer (FRP) Structures, which based on the experimental data included in this study exhibit average error percentages of $+184.6 \%$ and $+100.2 \%$, respectively. Conversely, the mean error for the proposed prediction model across all failure modes is calculated to be $-2.06 \%$, with a standard deviation of $9.15 \%$. Much of this associated error stems from the use of average values for the strength and stiffness properties of 
the test specimens obtained from a limited number of coupon tension tests. In design practice, more precise values for these properties would be provided for each specimen size and type by the manufacturer, which should result in greater accuracy in prediction of the critical flexural load capacity. Additional sources of error include variances in test setup and loading rate, as well as manufacturing inconsistencies.

\subsection{Critical LTB Capacity of GFRP Open Sections}

Pultruded GFRP channel and WF sections of a variety of sizes were tested under threepoint bending and then analyzed to determine the critical buckling load of these specimens. Testing was performed at numerous span lengths using multiple kinds of lateral bracing mechanisms placed at several different bracing positions along the test span. Observed variation in the plotted test data led to the development of a critical buckling load range defined by an upper and lower bound. These lower and upper bounds were selected based on slope changes observed during visual inspection of experimental data plots of vertical applied load versus 45 degree strain, and verified by plots of vertical applied load versus horizontal deflection. The acquired experimental data was then compared to two existing critical LTB load prediction models: one from the May 2013 draft of the ASCE/ACMA Pre-Standard for Load and Resistance Factor Design (LRFD) of Pultruded Fiber Reinforced Polymer (FRP) Structures, and one from the ASCE Structural Plastics Design Manual (1984). The results from analysis of these two models yielded highly inconsistent and inaccurate results, leading to the development of a more accurate and easy to apply critical LTB load prediction model. Detailed results from this testing and analysis can be found in Chapter 4. 


\subsubsection{WF Sections}

The proposed prediction model for the critical LTB load capacity of WF sections exhibits better accuracy and consistency than existing models for all tested specimens. By modifying the shape properties used in the classical elastic LTB equation, the proposed model predicted within $\pm 15 \%$ error of the experimental upper bound of the critical LTB load range for four different sizes of WF sections based on an average taken from a number of three point bending tests. This model also displayed accuracy by predicting within $+11.4 \%$ of the experimental upper bound of the critical LTB load range for a 12 " $\times 12$ " $\times 0.5$ " WF specimen tested in a four-point bend by Bendidi (1996). The mean error percentage for prediction of the upper bound of the critical LTB load range for WF sections using the proposed model is $+3.7 \%$, with a standard deviation of 11.4\%. In comparison, the existing critical LTB load prediction equations from the May 2013 draft of the ASCE/ACMA LRFD Pre-Standard and from the ASCE Structural Plastics Design Manual (1984), yielded mean error percentages for prediction of the upper bound of the critical LTB load range for WF sections of $+84.7 \%$, and $+161.8 \%$, respectively.

Due to shear lag, experimental data has shown that the strain in the flange is not uniform and not 100 percent of the peak bending strain, which explains why the effective flange width is used in calculations. Further analysis is required in order to better understand this effect and the resulting bending of the flanges for accurately representing $C_{w}, I_{y}$, and $J$ of a WF specimen. Furthermore, a variation of the corner stress concentration factor proposed for channel sections may need to be implemented to account for the added stiffness at the web-flange junctions.

A main source of error in this study stems from the use of average values for the strength and stiffness properties of the test specimens obtained from a limited number of coupon tension tests. In design, these values for the transverse and longitudinal elastic moduli, as well as the 
shear modulus, would be provided for each section size based on catalogued data provided by the manufacturer. Use of this data in applying the model should result in greater accuracy in predicting the critical LTB load capacity of pultruded GFRP wide flange specimens.

\subsubsection{Channel Sections}

The proposed prediction model for the critical LTB load capacity of channels exhibits better accuracy and consistency than existing models for all tested specimens. By modifying the calculations for the shape properties $C_{w}, I_{y}$, and $J$, for use in the classical elastic LTB equation and applying a corner stress concentration factor, $k$, to the thickness of the flange, the proposed yielded accurate prediction results for the experimental upper bound of the critical LTB load range for six channel sections based on a number of three point bending tests. Prediction fell within \pm 10 percent of the upper bound for nearly all of the sections tested, with the exception of the $10 " \times 2.75 " \times 0.5$ " channel section which exhibited -15.2 percent error. The mean error percentage for prediction of the upper bound of the critical LTB load range for channel sections using the proposed model is $-0.74 \%$, with a standard deviation of $8.6 \%$. In comparison, the existing critical LTB load prediction equations from the May 2013 draft of the ASCE/ACMA LRFD Pre-Standard and from the ASCE Structural Plastics Design Manual (1984), yielded mean error percentages for prediction of the upper bound of the critical LTB load range for channel sections of $-45.1 \%$, and $-22.2 \%$, respectively. Although the ASCE Plastics Manual yielded accurate prediction for some specimen sizes, it exhibited inaccurate results for others, resulting in an error percentage standard deviation of $32.7 \%$.

For this study, the influence of the effective flange width is taken to be negligible for channel specimens, resulting in more simplified calculations associated with this model. It is, however, likely that for larger specimens possessing longer channel legs that the effective flange 
width will need to be accounted for. Further analysis is also needed to determine the exact relationship between the torsional stiffness of the compression flange, $J_{f}$, and the constant, $\beta$, which could result in a direct equation for calculating the magnitude of corner stress concentration factor.

A main source of error in this study stems from the use of average values for the strength and stiffness properties of the test specimens obtained from a limited number of coupon tension tests. In design practices, characteristic values of these properties would be provided for each section size based on catalogued data provided by the manufacturer. Use of this data in applying the model should result in greater accuracy in predicting the critical LTB load capacity of pultruded GFRP channel specimens. Secondary sources of error include variances in test setup and loading rate, as well as manufacturing inconsistencies.

\subsubsection{Analysis of Lateral Constraint Mechanisms}

Prior to the initial stages of testing, a mechanism was fabricated allowing for $1 / 2$ inch thick steel bar, cut to about 65 percent of the depth of the web, to be held firmly against the web using threaded rod attached to a fixed steel angle using nuts. A second mechanism was considered using wooden blocks to brace the outside edges of the flanges by lodging the wood between the sample and a fixed steel angle using wooden construction shims. Neither mechanism proved to particularly effective in increasing the resistance to lateral-torsional buckling.

The first mechanism described, using steel bar pressed to the web, often resulted in a small, though insignificant, increase in critical LTB load capacity. However, this type of mechanism is difficult to achieve in practice because cross-bracing is placed between two beams which are each susceptible to lateral deformation. The second method described is more similar to common practice, but proved to be ineffective, mainly due to the rigidity of the wood being 
far less than that of the tested sample. This allowed for deformation in the wooden blocks resulting in slight movement and insufficient fixity at the lateral bracing positions. In a number of tests, the wooden construction shims fractured, allowing sudden unexpected movement at the lateral bracing positions.

A more efficient system would involve bracing the outer edges of the flanges and web using fixed steel or FRP lateral constraints. Based on experimental observation, larger and more rigid channel specimens had a tendency to roll on the large outer radius of the reentrant corner, allowing the strong-axis for bending of the specimen to move out of plane at the outer supports. Thus, by restraining movement in both the web and flanges, a more effective bracing mechanism is achieved. 


\section{REFERENCES}

American Association of State Highway and Transportation Officials (2012). AASHTO LRFD Bridge Design Specifications, 6th ed., FHWA/AASHTO, Washington, DC.

American Institute of Steel Construction (2001). LRFD Manual of Steel Construction, 3rd ed., AISC, Chicago, IL.

American Society of Civil Engineers (1984). Structural Plastics Design Manual, ASCE, Reston, VA.

American Society of Civil Engineers / American Composite Manufacturers Association (2010). "Pre-Standard for Load and Resistance Factor Design (LRFD) of Pultruded Fiber Reinforced Polymer (FRP) Structures.” Chapter 5.

Ascione, L., Giordano, A., and Spadea, S. (2011). "Lateral buckling of pultruded FRP beams." Composites: Part B, 42, 819-824.

Ascione, L., Berardi, V. P., Giordano, A., and Spadea, S. (2012). "Local Buckling Analysis of Pultruded FRP Thin-Walled Beams and Columns.” Proceedings of Composites in Civil Engineering (CICE), 1-10.

Ashby, M.F. (2005). Materials Selection in Mechanical Design, 3rd ed., ButterworthHeinermann, San Diego, CA, 285-295.

Bank, L. C. (1987). "Shear coefficients for thin-walled composite beams." Composite Structures, $8,47-61$.

Bank, L. C. (1989). "Flexural and Shear Moduli of Full-Section Fiber Reinforced Plastic (FRP) Pultruded Beams.” J. Test. Eval., 17(1), 40-45.

Bank, L. C. (2006). Composites for Construction: Structural Design with FRP Material. John Wiley \& Sons, Inc., Hoboken, NJ. 
Barbero, E. J. and Raftoyiannis, I. G. (1994). "Lateral and distortional buckling of pultruded Ibeams.” Compos. Struct., 27, 261-268.

Barbero, E. J. (2000). "Prediction of buckling-mode interaction in composite columns.” Mech. Compos. Mater. Struct., 7, 269-284.

Beer, F. P., Johnston, E. R., and DeWolf, J. T. (2002). Mechanics of Materials, 3rd ed., McGraw-Hill, New York, NY, 248-249, 473.

Bendidi, R. (1996). "Modeling and testing of FRP structural shapes under bending." M.S. thesis, West Virginia University, Morgantown, WV.

Bleich, F. (1952). Buckling of Metal Structures, McGraw-Hill Publishing Company, New York, NY.

Blodgett, O. W. (1966). Design of Welded Structures. James F. Lincoln Arc Welding Foundation, Cleveland, OH, 6.2-1-4.

Callister, W.D. (2007). Materials Science and Engineering: An Introduction, 7th ed., John Wiley \& Sons, New York, NY, 215-218.

Chen, D. and El-Hacha, R. (2012). "Buckling Analysis of Pultruded GFRP Hollow Box Beam." $6^{\text {th }}$ International Conference on FRP Composites in Civil Engineering, 1-7.

Clark, J. W. and Hill, H. N. (1960). “Lateral buckling of beams.” J. Struct. Div., ASCE, 86(ST7), 175-196.

Hart-Smith, L. J. (1989). “A New Approach to Fibrous Composite Laminate Strength Prediction." Eighth DOD/NASA/FAA Conference on Fibrous Composites in Structural Design, NASA CP-3087, Part 2, 663-693.

Johnson, A. F. (1985). "Simplified buckling analysis for RP beams and columns." $1^{\text {st }}$ European Conference on Composite Materials, Bordeaux, France, 541-549. 
Kassimali, A. (2012). Matrix Analysis of Structures, 2nd ed., Cengage Learning, Stamford, CT, $541-544$.

Kollár, L. P. (2002). "Discussion of 'Local buckling of composite FRP shapes by discrete plate analysis' by P. Qiao, J. F. Davalos, and J. Wang.” J. Struct. Eng., 128(8), 1091-1093.

Kollár, L. P. (2003). "Local Buckling of Fiber Reinforced Plastic Composite Structural Members with Open and Closed Cross Sections." J. Struct. Eng.-ASCE, 129(11), 1503-1513.

Lekhnitskii, S. G. (1968). Anisotropic Plates, Gordon and Breach Science Publishers, New York, NY.

Liang, R. and GangaRao, H. V. S. (2013). "Fiber-reinforced polymer (FRP) composites in environmental engineering applications." Developments in fiber-reinforced polymer (FRP) composites for civil engineering, N. Uddin, ed., Woodhead Publishing, Philadelphia, PA, 410-468.

Lopez-Anido, R. A., and GangaRao, H. V. S. (1996). "Warping Solution for Shear Lag in ThinWalled Orthotropic Composite Beams.” J. Eng. Mech., ASCE, 122(5), 449-457.

Mottram, J. T. (1991). "Evaluation of design analysis for pultruded fibre-reinforced polymeric box beams." The Structural Engineer, 69(11), 211-220.

Mottram, J. T. (1992). "Lateral-torsional buckling of a pultruded I-beam.” Composites, 23(2), $81-92$.

Nagaraj, V. and GangaRao, H. V. S. (1997). "Static Behavior of Pultruded GFRP Beams." J. Compos. Constr., ASCE, 1(3), 122-129.

Nguyen, T. T., Chan, T. M., and Mottram, J. T. (2014). "Lateral-torsional buckling resistance by testing for pultruded FRP beams under different loading and displacement boundary conditions." Composites: Part B, 60, 306-318. 
Palmer, D. W., Bank, L. C., and Gentry, T. R. (1998). "Progressive tearing failure of pultruded composite box beams: experiment and simulation." Compos. Sci. Technol., 58, 13531359.

Popov, E.P. (1954). Mechanics of Materials, 3rd ed., Prentice-Hall, Inc., New York, NY.

Qiao, P. (1997). "Analysis and design optimization of fiber-reinforced plastic (FRP) structural beams." Doctoral dissertation, West Virginia University, Morgantown, WV.

Qureshi, M. A. (2012). "Failure Behavior of Pultruded GFRP Members Under Combined Bending and Torsion.” Doctoral dissertation, West Virginia University, Morgantown, WV.

Roberts, T. M. and Al-Ubaidi, H. (2001). "Influence of shear deformation on restrained torsional warping of pultruded FRP bars of open cross-section.” Thin-Walled Struct., 39, 395-414.

Roberts, T. M. (2002). "Influence of shear deformation on buckling of pultruded fiber reinforced plastic profiles." J. Compos. Construct., 6(4), 241-248.

Roberts, T. M. and Masri, H. M. K. J. A. H. (2003). "Section Properties and Buckling Behavior of Pultruded FRP Profiles.” J. Reinf. Plast. Compos., 22, 1305-1317.

Salmon, C.G. and Johnson, J.E. (1996). Steel Structures: Design and Behavior, 4th ed., HarperCollins College Publishers, New York, NY.

Southwell, R. V. (1932). "On the analysis of experimental observations in problems of elastic stability." Proc. R. Soc. Lond. Ser. A., 135(828), 601-616.

Timoshenko, S.P. and Gere, J.M. (1961). Theory of Elastic Stability, 2nd ed., McGraw-Hill Book Company, Inc., New York, NY.

Timoshenko, S. and Young, D. H. (1962). Elements of Strength of Materials, 4th ed., D. Van Nostrand Company, Inc., Princeton, NJ, 125-133. 
Timoshenko, S. (1966). Strength of Materials Part II: Advanced Theory and Problems, 3rd ed., D. Van Nostrand Company, Inc., Princeton, NJ, 300-345.

Trefftz, E. (1922). “Über die Wirkung einer Abrundung auf die Torsionsspannungen in der inneren Ecke eines Winkeleisens.” Z. Angew. Math. Mech., 2(4), 263-267.

Vlasov, V. Z. (1961). Thin-walled Elastic Beams. Pergamon Press, New York, NY.

Young, W. C. (1989). Roark's Formulas for Stress and Strain, 6th ed., McGraw-Hill International, 201.

Zweben, C., Smith, W. S., and Wardle, M. W. (1979). "Test Methods for Fiber Tensile Strength, Composite Flexural Modulus, and Properties of Fabric-Reinforced Laminates.” Composite Materials: Testing and Design (Fifth Conference), ASTM STP 674, S. W. Tsai, Ed., American Society for Testing and Materials, 228-262. 


\section{APPENDIX A - SAMPLE CALCULATION OF PRINCIPAL STRAINS}

The purpose of Appendix A is to provide sample calculations of maximum shear strain, $\gamma_{\max }$, and the principal strain, $\varepsilon_{l}$, in the web for failure prediction of intermediate span-to-depth beams $(6 \leq L / h \leq 10)$. For an example, a 3 " $\times 3$ " $\times 0.25$ " sample tested at a span length, $L$, of 18 inches $(L / h=6)$ is considered. First, measure and record the total flange width $\left(b_{f}\right)$, total height $(h)$, wall thickness $(t)$, and inner corner radius $\left(r_{i}\right)$ of the specimen. In this example, $b_{f}=3$ in., $h$ $=3$ in., $t=0.25$ in., and $r_{i} \approx 0.0313$ in. Next, calculate the depth of the web of the specimen $\left(d_{w}\right)$. For a box section, $d_{w}$ is equal to $h-2 t$. For this example:

$$
d_{w}=3 \text { in. }-(2)(0.25 \text { in. })=2.5 \text { in. }
$$

Next, calculate the moment of inertia with respect to the axis of bending. For a box section, the strong-axis moment of inertia is calculated as shown in Equation (A-1).

$$
I_{x}=\frac{1}{12}\left(b_{f} h^{3}-b_{c} d_{w}^{3}\right)
$$

In which

$b_{f}=$ full width of flange (in.)

$h=$ total depth of section (in.)

$b_{c}=$ clear spacing of flange (in.)

$d_{w}=$ depth of web $=h-2 t$ (in.)

Plugging in dimensions of the example specimen cross-section, the moment of inertia is calculated to be 


$$
I_{x}=\frac{1}{12}\left[(3 \text { in. })(3 \text { in. })^{3}-(2.5 \text { in. })(2.5 \text { in. })^{3}\right]=3.4948 \text { in. }^{4}
$$

This is the extent of section properties necessary for the calculations. If material property data is not available from the manufacturer, material properties will need to be determined for the specimen under evaluation through coupon level tension testing. Material properties in the web necessary for principal strain calculations include the longitudinal elastic modulus $\left(E_{L}\right)$, Poisson's ratio $(v)$, and the shear elastic modulus $\left(G_{L T}\right)$. The laminate properties for the $3 " \times 3 " \times 0.25$ " sample used for these calculations are as follows:

$$
\begin{gathered}
E_{L}=5.12 \mathrm{Msi}=5,120,000 \mathrm{psi} \\
G_{L T}=0.49 \mathrm{Msi}=490,000 \mathrm{psi} \\
v=0.43
\end{gathered}
$$

The strains in the specimen can then be calculated from the shape and material properties. The longitudinal strain, $\varepsilon_{x x}$, the transverse strain, $\varepsilon_{y y}$, and the shear strain, $\gamma_{x y}$, are calculated using Equations (A-2) through (A-4)

$$
\begin{gathered}
\varepsilon_{x x}=\frac{P L c}{4 E_{L} I} \\
\varepsilon_{y y}=-v \varepsilon_{x x} \\
\gamma_{x y}=\frac{1.5 P}{2 t_{w} h G_{L T}} \times \frac{1}{2}
\end{gathered}
$$

In which

$P=$ applied load (lbf)

$L=$ total span length (in.)

$c=$ distance to the neutral axis from inside of flange (in.) $=d_{w} / 2$ for symmetric box sections 
$E_{L}=$ characteristic value of the longitudinal modulus (psi)

$v_{L T}=$ characteristic value of Poisson's ratio

$G_{L T}=$ characteristic value of the in-plane shear modulus (psi)

$t_{w}=$ thickness of one web (in.)

$h=$ total height of section (in.)

It should be noted that the factor of is applied to the shear strain equation due to the presence of two webs in a box section. Based on experimental data as shown in Section 3.6.2, the critical flexural load of this specimen is taken as $5749 \mathrm{lbf}$. Taking this value as the applied load, $P$, the strain components in the example specimen, calculated using Equations (A-2) through (A4), are found to be

$$
\begin{gathered}
\varepsilon_{x x}=\frac{(5749 \mathrm{lbf})(18 \mathrm{in} .)(2.5 \mathrm{in} . / 2)}{(4)(5,120,000 \mathrm{psi})\left(3.4948 \mathrm{in} .^{4}\right)}=0.001807 \\
\varepsilon_{y y}=-(0.43)(0.001807)=-0.000777 \\
\gamma_{x y}=\frac{1.5(5749 \mathrm{lbf})}{(2)(0.25 \mathrm{in} .)(3 \mathrm{in} .)(500,000 \mathrm{psi})} \times \frac{1}{2}=0.005749
\end{gathered}
$$

From these values, the center and radius of Mohr's Circle for strain can be calculated using Equations (A-5) and (A-6).

$$
\begin{gathered}
C=\varepsilon_{\text {avg }}=\frac{\varepsilon_{x x}+\varepsilon_{y y}}{2} \\
R=\frac{\gamma_{\max }}{2}=\sqrt{\left(\frac{\varepsilon_{x x}-\varepsilon_{y y}}{2}\right)^{2}+\left(\frac{\gamma_{x y}}{2}\right)^{2}}
\end{gathered}
$$

Substituting in the calculated values for this specimen, the center and radius of Mohr's Circle for strain are calculated to be 


$$
\begin{gathered}
C=\varepsilon_{\text {avg }}=\frac{0.001807+(-0.000777)}{2}=0.000515 \\
R=\frac{\gamma_{\max }}{2}=\sqrt{\left[\frac{0.001807-(-0.000777)}{2}\right]^{2}+\left(\frac{0.005749}{2}\right)^{2}}=0.003152
\end{gathered}
$$

From these values the maximum shear strain, $\gamma_{\max }$, and the principal strain, $\varepsilon_{l}$, in the web can be calculated using Equations (A-7) and (A-8)

$$
\begin{aligned}
& \gamma_{\max }=2 R \\
& \varepsilon_{1}=C+R
\end{aligned}
$$

Plugging in the calculated values for the 3 " $\times 3$ " $\times 0.25$ " sample, the maximum shear strain and maximum principal strain in the web of this specimen are found to be

$$
\begin{gathered}
\gamma_{\max }=(2)(0.003152)=0.006304=6,304 \mu \varepsilon \\
\varepsilon_{1}=0.000515+0.003152=0.003667=3,667 \mu \varepsilon
\end{gathered}
$$

To complete the prediction model presented in Section 3.3.2, these values must be multiplied by the stress intensity factor, SIF, as calculated using Equation (3-14).

$$
\begin{gathered}
\operatorname{SIF}=\frac{k d}{\sqrt{L t}}=\frac{\sqrt[3]{(0.25 \text { in. } / 0.0313 \text { in. })}(3 \text { in. })}{\sqrt{(18 \text { in. })(0.25 \text { in. })}}=2.8269 \\
\gamma_{\max } \times \operatorname{SIF}=(0.006304)(2.8269)=0.017821=17,821 \mu \varepsilon \\
\varepsilon_{1} \times \operatorname{SIF}=(0.003667)(2.8269)=0.010366=10,366 \mu \varepsilon
\end{gathered}
$$




\section{APPENDIX B - DERIVATION OF STRESS INTENSITY FACTOR}

The shape factor at failure (not service condition) under bending, $\varphi_{B}^{f}$, which is different from the shape factor within elastic response ranges, is given as a function of the $h / t$ ratio using Equations (B-1) and (B-2) from Ashby (2005)

$$
\begin{gathered}
\varphi_{B}^{f}=\frac{1}{\sqrt{2}} \sqrt{\frac{h}{t}} \frac{\left(1+3 b_{f} / h\right)}{\left(1+b_{f} / h\right)^{3 / 2}} \\
E I=\varphi_{B}^{f} E I_{0}
\end{gathered}
$$

For simplicity, let the quantity $\frac{\left(1+3 b_{f} / h\right)}{\left(1+b_{f} / h\right)^{3 / 2}}$ be represented by the variable, $\alpha$. Then, by combining Equations (B-1) and (B-2), it can be shown that

$$
E I=\frac{\alpha}{\sqrt{2}} \sqrt{\frac{h}{t}} E I_{0}
$$

Equation (B-4) is used to calculate the maximum principal strain in a continuum. From this, it can be shown that the strain is a function of the span-to-depth $(L / h)$ ratio.

$$
\varepsilon_{\max }=-\frac{\varepsilon_{x}+\varepsilon_{y}}{2} \pm \sqrt{\left(\frac{\varepsilon_{x}-\varepsilon_{y}}{2}\right)^{2}+\left(\frac{\gamma_{x y}}{2}\right)^{2}}
$$

In which

$\varepsilon_{x}=$ longitudinal tensile strain

$\varepsilon_{y}=$ tranverse strain

$\gamma_{x y}=$ shear strain 
Based on experimental findings, it can be assumed that $\varepsilon_{y}=v \varepsilon_{x}$, where $v$ is Possion's ratio, which for simplicity is assumed to be equal to 0.3 . By substituting this value into Equation (B-4), the equation becomes

$$
\varepsilon_{\max }=-0.65 \varepsilon_{x}+\sqrt{\left(0.35 \varepsilon_{x}\right)^{2}+\left(\frac{\gamma_{x y}}{2}\right)^{2}}
$$

The longitudinal tensile strain, $\varepsilon_{x}$, can be expressed by taking the classic flexure formula and including the longitudinal elastic modulus, $E_{x}$, in the denominator. The result is shown in Equation (B-6).

$$
\varepsilon_{x}=\frac{M c}{E_{x} I}
$$

Assuming, for simplicity, that for thin-walled box sections $b_{c} \approx 0.8 b_{f}$ and $d_{w} \approx 0.9 h$, where $b_{c}=$ clear spacing of the flange, $b_{f}=$ total width of the flange, $d_{w}=$ clear depth of the web, and $h=$ total height of the box section, the moment of inertia of the section can be approximated as shown in Equation (B-7).

$$
I=\frac{b_{f} h^{3}-b_{c} d_{w}^{3}}{12} \cong \frac{b_{f} h^{3}-\left(0.8 b_{f}\right)(0.9 h)^{3}}{12} \cong 0.0347 b_{f} h^{3}
$$

By substituting Equation (B-7) into (B-6), $\varepsilon_{x}$ can be rewritten for a three point load case as shown in Equation (B-8).

$$
\varepsilon_{x}=\frac{(P L / 4)(h / 2)}{E_{x}\left(0.0347 b_{f} h^{3}\right)} \cong \frac{P L}{0.2779 E_{x} b_{f} h^{2}}
$$


Substituting Equation (B-8) into Equation (B-5) and simplifying leads to

$$
\varepsilon_{\max } \cong \frac{-2.34 P L}{E_{x} b_{f} h^{2}}+\sqrt{\left(\frac{1.26 P L}{E_{x} b_{f} h^{2}}\right)^{2}+\left(\frac{\gamma_{x y}}{2}\right)^{2}}
$$

Next, multiply both sides of the equation by $E_{x}$. In order to do so, the shape factor as shown in Equation (B-3) must be applied to the shear strain term in order to relate the elastic modulus, $E_{x}$, to the shear modulus, $G$. The moment of inertia may be factored out of each of the terms, thus leaving the equation as

$$
E_{x} \varepsilon_{\max } \cong \frac{-2.34 P L}{b_{f} h^{2}}+\sqrt{\left(\frac{1.26 P L}{b_{f} h^{2}}\right)^{2}+\left(\frac{E_{x} \gamma_{x y} \alpha \sqrt{h / t}}{2 \sqrt{2}}\right)^{2}}
$$

Then, isolate the square root function on the right side of Equation (B-10). Taking the square of both sides of this equation then reveals Equation (B-11).

$$
\begin{aligned}
& E_{x}^{2} \varepsilon_{\text {max }}^{2}+4.68 E_{x} \varepsilon_{\max }\left(\frac{P}{b_{f} h}\right)\left(\frac{L}{h}\right)+5.48\left(\frac{P}{b_{f} h}\right)^{2}\left(\frac{L}{h}\right)^{2} \\
& =1.26\left(\frac{P}{b_{f} h}\right)^{2}\left(\frac{L}{h}\right)^{2}+\frac{E_{x}^{2} \gamma_{x y}^{2} \alpha^{2}}{8}\left(\frac{h}{t}\right)
\end{aligned}
$$

Combining like terms yields Equation (B-12).

$$
E_{x}^{2} \varepsilon_{\max }^{2}+4.68 E_{x} \varepsilon_{\max }\left(\frac{P}{b_{f} h}\right)\left(\frac{L}{h}\right)+4.22\left(\frac{P}{b_{f} h}\right)^{2}\left(\frac{L}{h}\right)^{2}=\frac{E_{x}^{2} \gamma_{x y}^{2} \alpha^{2}}{8}\left(\frac{h}{t}\right)
$$

Dividing both sides of the equation by $E_{x}^{2}$ leads to Equation (B-13).

$$
\varepsilon_{\max }^{2}+\left(\frac{4.68 \varepsilon_{\max }}{E_{x}}\right)\left(\frac{P}{b_{f} h}\right)\left(\frac{L}{h}\right)+\left(\frac{4.22}{E_{x}^{2}}\right)\left(\frac{P}{b_{f} h}\right)^{2}\left(\frac{L}{h}\right)^{2}=\frac{\gamma_{x y}^{2} \alpha^{2}}{8}\left(\frac{h}{t}\right)
$$


Because $\varepsilon_{\max }^{2}$ is very small and $E_{x}^{2}$ is very large, the first and third terms on the left side Equation (B-13) can be considered negligible. For greater accuracy, the third term could be retained, but overall it does not contribute much to $\gamma_{x y}$. By removing these terms, Equation (B13) can be rewritten as shown in Equation (B-14).

$$
\left(\frac{4.68 \varepsilon_{\max }}{E_{x}}\right)\left(\frac{P}{b_{f} h}\right)\left(\frac{L}{h}\right)=\frac{\gamma_{x y}^{2} \alpha^{2}}{8}\left(\frac{h}{t}\right)
$$

By isolating the shear strain, $\gamma_{x y}$, to one side of the equation, it can be shown in Equation (B-15) that the shear strain is a function of the stress intensity factor component $\frac{h}{\sqrt{L t}}$, which is found in Equation (3-13).

$$
\gamma_{x y} \cong 6.12 \sqrt{\frac{\varepsilon_{\max }}{E_{x} \alpha^{2}}} \sqrt{\frac{P}{b_{f} h}} \frac{\sqrt{L t}}{h}, \text { where } \alpha=\frac{\left(1+3 b_{f} / h\right)}{\left(1+b_{f} / h\right)^{3 / 2}}
$$




\section{APPENDIX C - COUPON LEVEL TENSION TEST RESULTS}

Many of the equations proposed and discussed in Chapters 3 and 4 are inherently dependent on the laminate properties of the full-size GFRP member. Due to the high cost of fullsize member testing, these properties are obtained by coupon level testing. The goal of this testing was to acquire average values for the longitudinal elastic modulus of the flange and web, transverse elastic modulus of the flange and web, and characteristic longitudinal strength, $F_{L f}$, in the flange of specimens included in this study. For determining the laminate properties in the longitudinal direction, specimens were cut along the pultruded fiber axis. Conversely, for determining the transverse elastic modulus, specimens were cut perpendicular to the pultruded fiber axis, so that the fibers were in the transverse direction with respect to the length of the samples. Additionally, coupon samples cut from the web of both a $6 " \times 6 " \times 0.375^{\prime \prime}$ and a $6 " \times 4 " \times 0.25 "$ closed section at an angle of 35 degrees with respect to the pultruded fiber axis were tested to confirm the range of principal strain to failure in the tension zone of the web observed for square and rectangular GFRP box sections tested with the threshold range of $6 \leq$ $L / h \leq 10$, as discussed in Section 3.3.2.

Tension testing at the coupon level for thin-walled laminate composites is performed according to ASTM Standard D3039/D3039M-08, Standard Test Method for Tensile Properties of Polymer Matrix Composite Materials. Coupon samples tested for the purposes of this study were cut from pultruded GFRP box, channel, and WF sections. It should, however, be noted that due to geometric constraints, length requirements set forth by ASTM D3039 could not always be met. Each of the coupon samples used to calculate the modulus of elasticity were equipped with one $350 \mathrm{ohm}$ linear strain gage having a $1 / 4$ inch gage length, placed at the center of the length and width of the sample in the longitudinal direction. Some samples were also tested using an 
extensiometer to record strain values, but this piece of equipment must be removed from the sample prior to failure. The extensiometer has also been shown in the past by WVU-CFC researchers to be less accurate than strain gages. All coupon level testing was performed using either an Instron 8501 or Instron Industrial Series 1000HDX Model Servohydraulic Testing Machine. Test samples were fixed within two grips, one on each end of the specimen, being sure to align the long axis of the specimen with the test direction. One end of the sample was held stationary, while the hydraulic actuator was used to pull on the other end at a standard head displacement rate of $0.05 \mathrm{in} . / \mathrm{min}$.. Testing was performed until rupture, at which point the mode of failure is to be evaluated. It should be noted that any specimen that breaks at an obvious flaw, or that fails within one specimen width of the grip, should not be evaluated in calculating the laminate properties. Based on ASTM D3039, the elastic modulus is to be taken as the tensile chord modulus of elasticity, equal to the slope of the stress-strain curve between $1000 \mu \varepsilon$ and $3000 \mu \varepsilon$. A simple evaluation of the accuracy of the tensile chord modulus of elasticity can be obtained by calculating the coefficient of determination $\left(R^{2}\right)$, which represents the how well the experimental data matches with the slope indicated by the calculated E modulus.

The test results shown in Table B-1 are from tension testing of coupon samples cut in the longitudinal direction from the flange of a $3 " \times 3 " \times 0.25 "$ box section. These samples were cut to a width of $3 / 4$ inch. The tensile chord modulus of elasticity was calculated for use in the prediction model shown in Section 3.3. Because the results for specimen $3 \times 3 \times 0.25-1$ indicated that the sample was damaged, the longitudinal modulus of elasticity of this box section is taken to be 5.12 , which is the average of the results of the two other specimens which were equipped with strain gages. Similarly, the longitudinal strength in the flange is taken to be $48.7 \mathrm{ksi}$ for the 
$3^{\prime \prime} \times 3^{\prime \prime} \times 0.25$ " box section based on the results shown in Table C-1. Some typical failure modes exhibited during this testing are shown in Figure C-1.

Table C-1 Tension test results for longitudinal coupon samples cut from flange of a $3 " \times 3 " \times 0.25 "$ box section

\begin{tabular}{|c|c|c|c|c|c|l|}
\hline Sample & $\begin{array}{c}\text { Maximum } \\
\text { Deflection } \\
\text { (in.) }\end{array}$ & $\begin{array}{c}\text { Peak } \\
\text { Load } \\
\text { (lbf) }\end{array}$ & $\begin{array}{c}\text { Stress at } \\
\text { Peak Load } \\
\text { (psi) }\end{array}$ & $\begin{array}{c}\text { Strain Gage } \\
\text { Modulus } \\
\text { (Msi) }\end{array}$ & $\mathbf{R}_{\text {gage }}^{2}$ & Notes \\
\hline \hline $\mathbf{3 \times 3 \times 0 . 2 5 - 4}$ & 0.186 & 8357 & 41167.49 & 3.7819 & $\theta .9993$ & Sample damaged prior to testing \\
\hline $\mathbf{3 \times 3 \times 0 . 2 5 - 2}$ & 0.2395 & 10618 & 54536.56 & 5.1679 & 0.9986 & \\
\hline $\mathbf{3 \times 3 \times 0 . 2 5 - 3}$ & 0.239 & 10866 & 54883.72 & 5.0749 & 0.9991 & \\
\hline $\mathbf{3 \times 3 \times 0 . 2 5 - 4}$ & 0.2262 & 8720 & 47676.33 & N/A & N/A & No strain recorded \\
\hline $\mathbf{3 \times 3 \times 0 . 2 5 - 5}$ & 0.2115 & 8816 & 43471.4 & N/A & N/A & No strain recorded \\
\hline $\mathbf{3 \times 3 \times 0 . 2 5 - 6}$ & 0.202 & 8942 & 50691.61 & N/A & N/A & No strain recorded \\
\hline
\end{tabular}

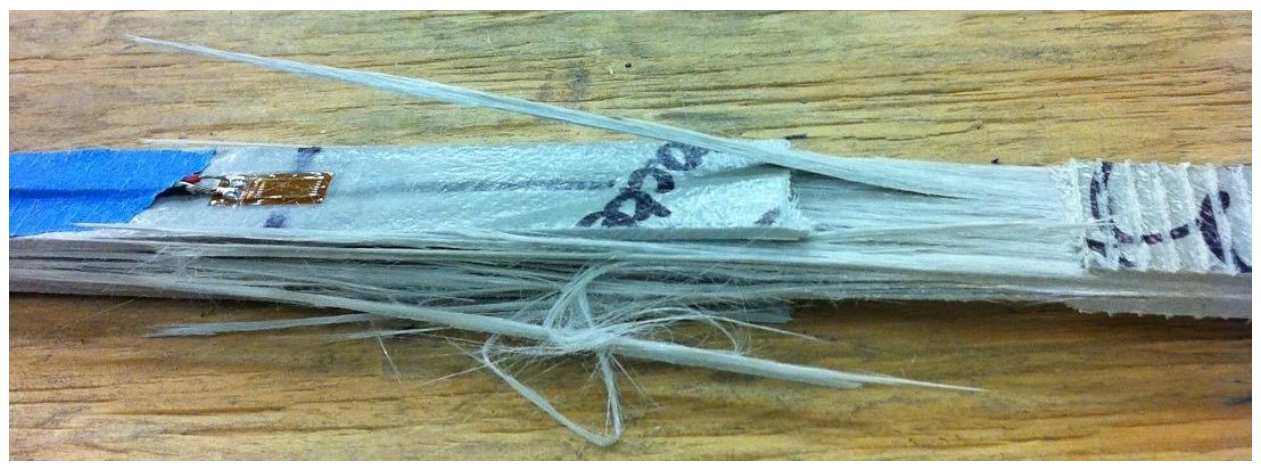

(a)

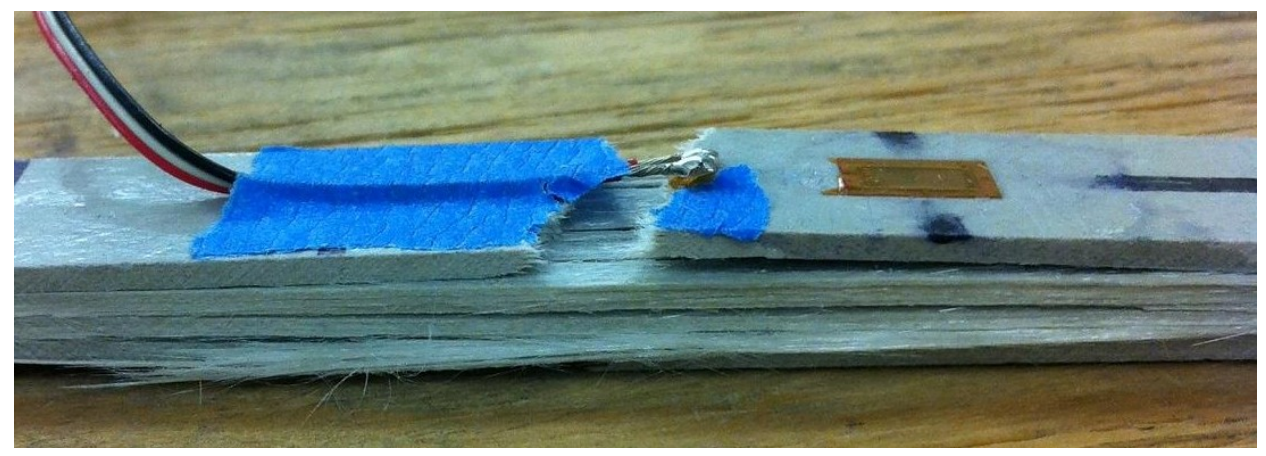

(b)

Figure C-1 (a) and (b) Typical failure modes of longitudinal coupon samples cut from the flange of a $3 " \times 3 " \times 0.25 "$ box section 
As previously mentioned, additional coupon samples were cut from the web of both a $6^{\prime \prime} \times 6 " \times 0.375^{\prime \prime}$ and a $6 " \times 4 " \times 0.25^{\prime \prime}$ closed section at an angle of 35 degrees with respect to the pultruded fiber axis. These samples were tested in tension to confirm the range of principal strain to failure observed during full-size section testing within the threshold range of $6 \leq L / h \leq 10$. The strains to failure obtained through this testing matched well with those captured during full-size section testing by a strain gage installed in the tension zone of the web at an angle of approximately 40 degrees with respect to the pultruded fiber axis. The chord elastic modulus was also calculated for these samples based on strain resins from both an extensiometer, as well as strain gages on a few of the samples. The results are shown in Table C-2, with photos from the testing shown in Figures C-2 and C-3.

Table C-2 Tension test results for transverse coupon samples cut from the web of box sections at a $35^{\circ}$ angle with respect the pultruded fiber axis

\begin{tabular}{|c|c|c|c|c|c|c|c|c|}
\hline Sample & $\begin{array}{c}\text { Peak } \\
\text { Load } \\
\text { (lbf) }\end{array}$ & $\begin{array}{c}\text { Stress at } \\
\text { Peak } \\
\text { Load } \\
\text { (psi) }\end{array}$ & $\begin{array}{l}\text { Extensiometer } \\
\text { Modulus (Msi) }\end{array}$ & $\mathbf{R}_{\text {extens }}^{2}$ & $\begin{array}{c}\text { Strain } \\
\text { Gage } \\
\text { Modulus } \\
\text { (Msi) }\end{array}$ & $\mathbf{R}_{\text {gage }}^{2}$ & $\begin{array}{c}\text { Strain at } \\
\text { Failure } \\
(\mu \varepsilon)\end{array}$ & Notes \\
\hline $6 \times 4-1$ & 2360.7 & 12349.0 & 1.2807 & 0.9988 & 1.4446 & 0.9988 & 11744.45 & \\
\hline $6 \times 4-2$ & 2472.9 & 13121.5 & 1.3924 & 0.9991 & 1.4117 & 0.9995 & 12012.82 & \\
\hline $6 \times 4-3$ & 2377.8 & 12411.3 & 1.3700 & 0.9993 & $\mathrm{~N} / \mathrm{A}$ & $\mathrm{N} / \mathrm{A}$ & N/A & Failed at grip \\
\hline $6 \times 4-4$ & 2432.3 & 12807.4 & 1.2948 & 0.9994 & N/A & N/A & N/A & \\
\hline $6 \times 4-5$ & 2456.9 & 13020.4 & 1.3919 & 0.9992 & $\mathrm{~N} / \mathrm{A}$ & $\mathrm{N} / \mathrm{A}$ & $\mathrm{N} / \mathrm{A}$ & Failed at grip \\
\hline $6 \times 4-6$ & 2500.3 & 13176.4 & 1.2896 & 0.9993 & N/A & $\mathrm{N} / \mathrm{A}$ & $\mathrm{N} / \mathrm{A}$ & \\
\hline $6 \times 4-7$ & 2557.4 & 13528.3 & 1.3844 & 0.9992 & N/A & N/A & N/A & \\
\hline $6 \times 4-8$ & 2204.1 & 11550.3 & 1.3558 & 0.9990 & N/A & $\mathrm{N} / \mathrm{A}$ & N/A & Failed at grip \\
\hline $6 \times 4-9$ & 2331.6 & 12658.6 & 1.3831 & 0.9989 & N/A & N/A & N/A & Failed at grip \\
\hline $6 \times 6-1$ & 2664.0 & 9178.1 & 1.0065 & 0.9779 & 1.3768 & 0.9989 & 8734.23 & \\
\hline $6 \times 6-2$ & 2808.7 & 9697.9 & 1.2015 & 0.9931 & 1.1881 & 0.9980 & 12525.57 & \\
\hline $6 \times 6-3$ & 2452.2 & 8406.2 & 1.2594 & 0.9802 & N/A & N/A & N/A & \\
\hline $6 \times 6-4$ & 2306.6 & 7910.2 & 1.0392 & 0.9935 & $\mathrm{~N} / \mathrm{A}$ & $\mathrm{N} / \mathrm{A}$ & N/A & Failed at grip \\
\hline $6 \times 6-5$ & 2257.8 & 7762.0 & 1.0336 & 0.9916 & N/A & N/A & N/A & \\
\hline $6 \times 6-6$ & 2158.3 & 7462.0 & 1.1339 & 0.9947 & N/A & N/A & N/A & Failed at grip \\
\hline $6 \times 6-7$ & 2335.1 & 8128.9 & 1.0853 & 0.9901 & N/A & $\mathrm{N} / \mathrm{A}$ & $\mathrm{N} / \mathrm{A}$ & \\
\hline $6 \times 6-8$ & 2659.8 & 9157.8 & 1.2160 & 0.9943 & N/A & N/A & N/A & \\
\hline
\end{tabular}




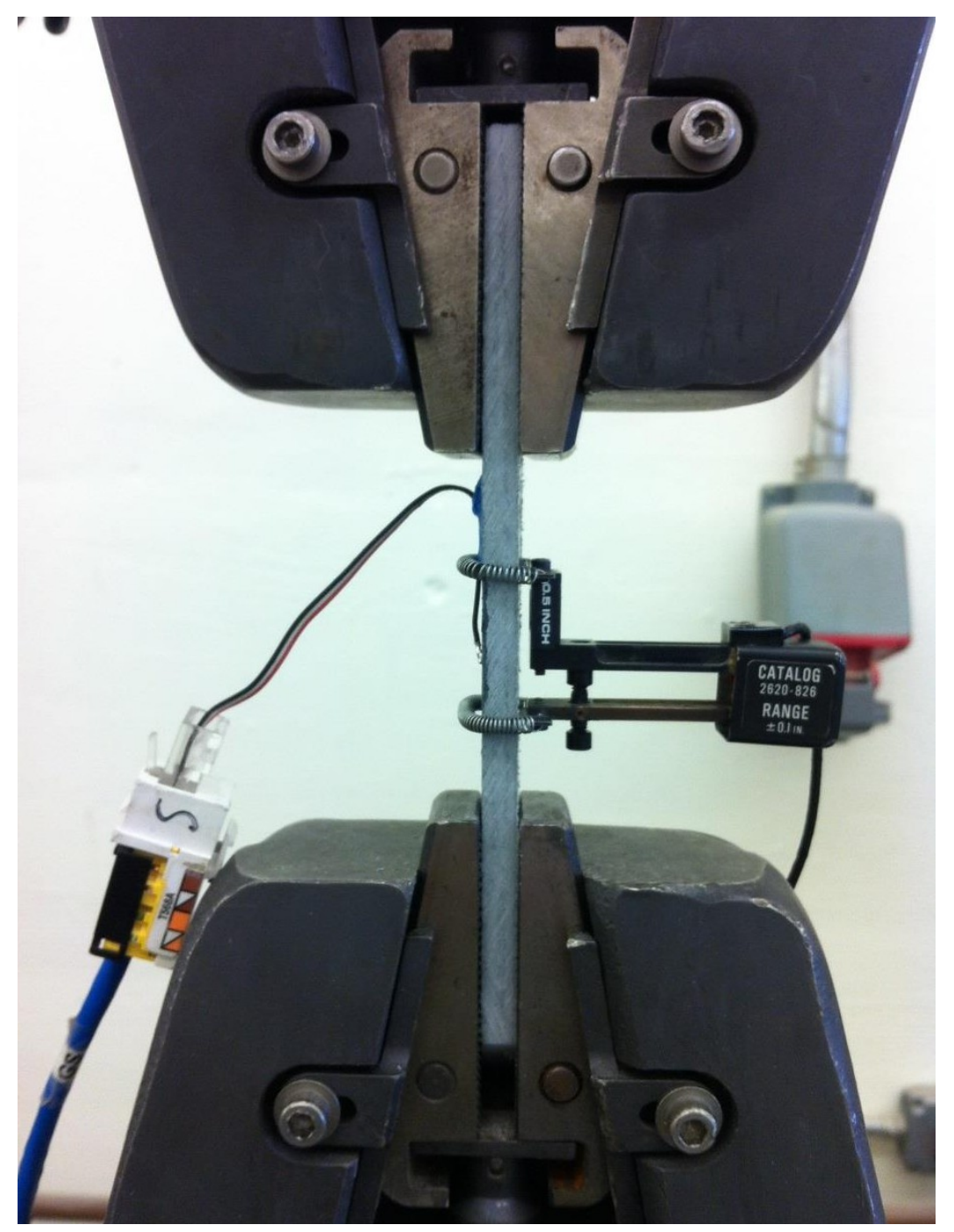

Figure C-2 Specimen 6x4-2 undergoing tension test equipped with strain gage and extensiometer

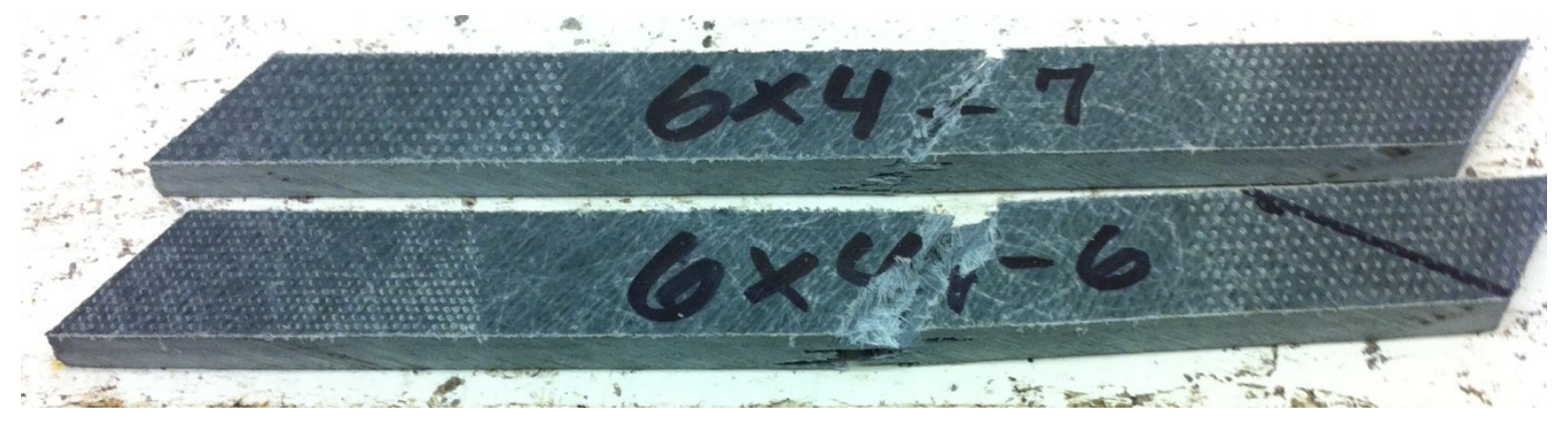

Figure C-3 Typical failure mode exhibited by tension coupon samples cut at an angle of 35 degrees with respect to the pultruded fiber axis 
The final set of coupon samples tested by the author was cut in the transverse direction from the webs of open sections. The results from these transverse coupon samples are shown in Table B-3. The samples are labelled according to the specimen from which they were cut, where $\mathrm{CW}$ corresponds to the web of the $10 " \times 2.75$ " $\times 0.5$ " channel specimen and IW corresponds to the web of the 10 " $\times 5$ " $\times 0.5$ " WF section. The first number in the label represents the width of the sample, i.e., 0.5 in. or 1 in. width. The second number in the label is the sample number, with samples from each group labelled 1 through 5. Two of the samples experienced cracking which was audible during placement into the fixture. These samples are noted within Table C-3, although results suggest that the damage was not significant. One sample (IW-0.5-1) was significantly damaged during placement into the fixture and was not tested, as shown in the table. The chord elastic modulus was calculated according to ASTM D3039 based on strain readings from a strain gage installed on each of the specimens, as shown in Table C-3. Figure C4 shows acceptable failure modes observed during this testing. An example of a typical tensile stress-strain curve resulting from this testing can be shown in Figure C-5. Notice that the tensile elastic modulus is calculated in the linear region prior to the transition region.

The resulting average elastic modulus, $\mathrm{E}$, for each of the sample groups is shown in Table C-4. As shown by the results, the elastic modulus in the transverse direction of the web is larger for WF sections as compared to channel specimens. This is due to a large fiber-volume fraction characteristic of the web of pultruded WF sections. The transverse elastic moduli results from this testing validated the average value used during development of the critical LTB load prediction model. 
Table C-3 Tension test results for coupon samples cut from the web of channel and WF sections

\begin{tabular}{|c|c|c|c|c|c|l|}
\hline Sample & $\begin{array}{c}\text { Maximum } \\
\text { Deflection } \\
\text { (in) }\end{array}$ & $\begin{array}{c}\text { Peak } \\
\text { Load } \\
\text { (lbf) }\end{array}$ & $\begin{array}{c}\text { Stress } \\
\text { at Peak } \\
\text { Load } \\
\text { (psi) }\end{array}$ & $\begin{array}{c}\text { Strain } \\
\text { Gage } \\
\text { Modulus } \\
\text { (Msi) }\end{array}$ & $\mathbf{R}_{\text {gage }}^{2}$ & Notes \\
\hline \hline CW-1-1 & 0.2089 & 6695.6 & 13311.4 & 1.4421 & 0.9922 & \\
\hline CW-1-2 & 0.2177 & 6865.1 & 13716.5 & 1.1095 & 0.9406 & \\
\hline CW-1-3 & 0.2324 & 6751.0 & 13441.4 & 1.0289 & 0.9396 & \\
\hline CW-1-4 & 0.2367 & 6787.9 & 13603.0 & 1.7087 & 0.9007 & \\
\hline CW-1-5 & 0.235 & 6849.6 & 13676.3 & 1.5016 & 0.9589 & \\
\hline CW-0.5-1 & 0.1973 & 3004.9 & 11940.0 & 1.6890 & 0.9379 & Sample was cracked placing into the fixture \\
\hline CW-0.5-2 & 0.2035 & 3141.4 & 12482.1 & 1.5349 & 0.9927 & Sample was cracked placing into the fixture \\
\hline CW-0.5-3 & 0.1885 & 2758.4 & 11030.3 & 1.8030 & 0.9475 & \\
\hline CW-0.5-4 & 0.2287 & 3376.2 & 13352.9 & 1.4436 & 0.9630 & \\
\hline CW-0.5-5 & 0.215 & 3410.8 & 13427.6 & 1.3072 & 0.9961 & \\
\hline IW-1-1 & 0.279 & 11398.8 & 22260.7 & 1.8145 & 0.9636 & \\
\hline IW-1-2 & 0.2831 & 11331.5 & 22009.1 & 1.9167 & 0.9886 & \\
\hline IW-1-3 & 0.2794 & 11233.6 & 21974.5 & 2.0026 & 0.9650 & \\
\hline IW-1-4 & 0.2985 & 11773.7 & 22898.9 & 2.0418 & 0.9690 & \\
\hline IW-1-5 & 0.2924 & 11811.0 & 23050.7 & 2.1311 & 0.9940 & \\
\hline IW-0.5-1 & --- & --- & --- & --- & --- & Sample was damaged placing into the fixture \\
\hline IW-0.5-2 & 0.2797 & 5101.9 & 20288.6 & 1.7808 & 0.9631 & \\
\hline IW-0.5-3 & 0.2645 & 4974.7 & 19659.7 & 1.8918 & 0.9701 & \\
\hline IW-0.5-4 & 0.2619 & 4715.2 & 18613.6 & 1.7431 & 0.9132 & \\
\hline IW-0.5-5 & 0.277 & 4949.3 & 19638.4 & 1.7503 & 0.9577 & \\
\hline
\end{tabular}

Table C-4 Averaged tension test results by specimen group for coupon samples cut from the web of channel and WF sections

\begin{tabular}{|c|c|c|c|c|}
\hline Sample & $\begin{array}{c}\text { Average } \\
\text { Maximum } \\
\text { Deflection } \\
\text { (in) }\end{array}$ & $\begin{array}{c}\text { Average } \\
\text { Peak } \\
\text { Load } \\
\text { (lbf) }\end{array}$ & $\begin{array}{c}\text { Average } \\
\text { Stress at } \\
\text { Peak } \\
\text { Load } \\
\text { (psi) }\end{array}$ & $\begin{array}{c}\text { Average } \\
\text { Elastic } \\
\text { Modulus } \\
\text { (Msi) }\end{array}$ \\
\hline \hline CW-1 & 0.2261 & 6789.8 & 13549.7 & 1.3581 \\
\hline CW-0.5 & 0.2066 & 3138.3 & 12446.6 & 1.5555 \\
\hline IW-1 & 0.2865 & 11509.7 & 22438.8 & 1.9813 \\
\hline IW-0.5 & 0.2708 & 4935.3 & 19550.1 & 1.7915 \\
\hline
\end{tabular}




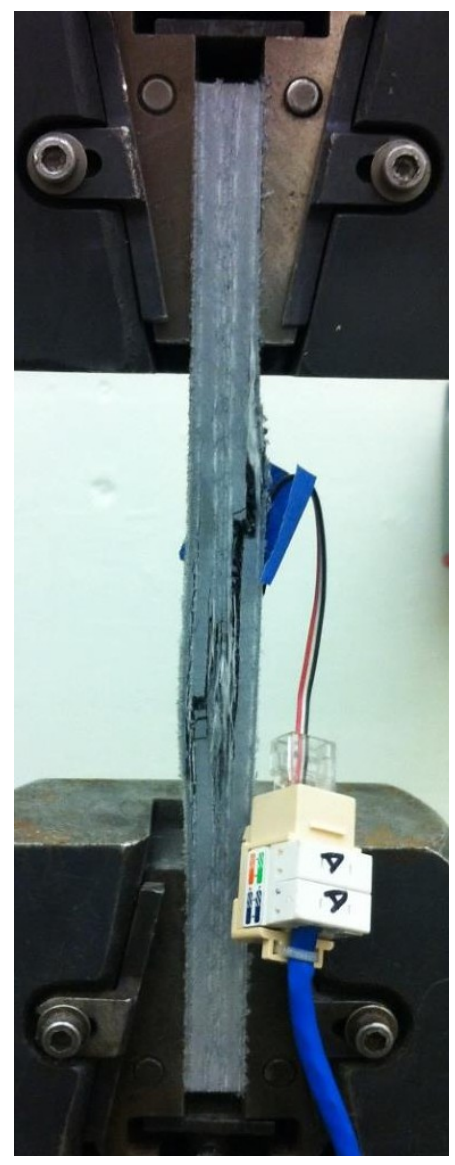

(a)

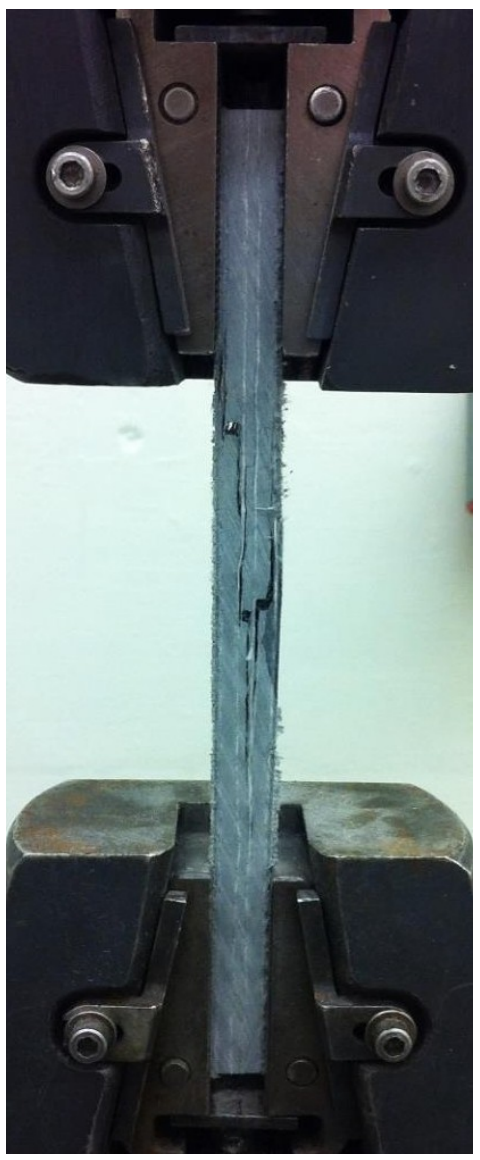

(b)

Figure C-4 (a) and (b) Typical angled failure modes observed during tensile testing of transverse coupon samples cut from the webs of open GFRP sections

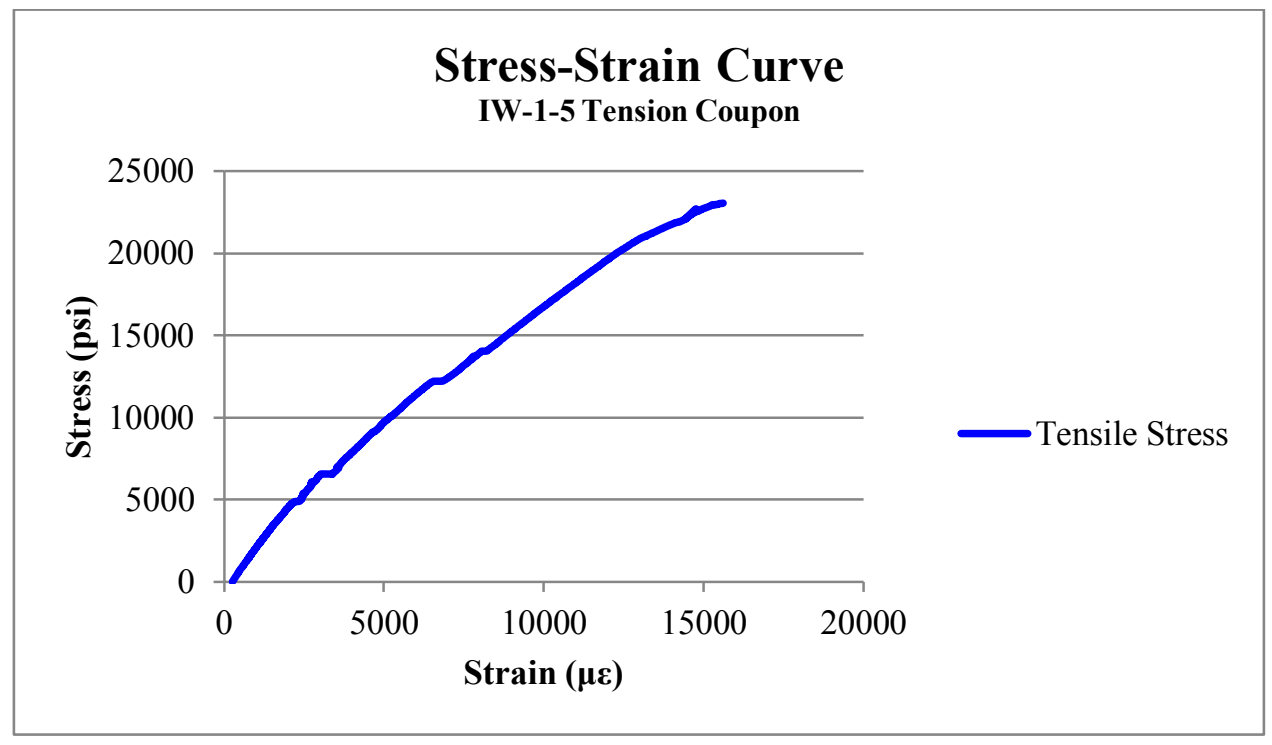

Figure C-5 Tensile stress-strain curve of IW-1-5 transverse coupon sample 


\section{APPENDIX D - PLOTS USED FOR DETERMINATION OF CRITICAL LTB LOAD RANGE}

This appendix serves to show how the lower and upper bounds of the critical LTB load range was determined based on plotted experimental data, similar to Figure 4-6 (a) and (b). The critical LTB load range was developed by evaluating the experimental test data based on what was considered to be the worst case scenario meaning the specimen was tested at the maximum span length with midspan loading, and no intermediate lateral bracing.

As shown in the plots following this commentary, development of the critical LTB load range is performed by evaluating only plots of vertical applied load versus 45 degree strain and vertical applied load versus horizontal deflection. In general, plots of strain measured at 45 degrees at mid-depth of the web will be linear leading up to the point at which buckling initiates. Similarly, the horizontal deflection should show minor lateral deflection up until the point of buckling, where the deflection will suddenly become more significant. It should be noted that in many tests, the measurable range of the horizontal LVDTs $( \pm 0.3 \mathrm{in}$.) was exceeded, leading the plot of load versus horizontal deflection to go vertical at this point. By tracing the initial linear portion of these plots, an initial minor change in slope can be identified - this point serves as the lower bound of the critical LTB load range. Then, by tracing the linearity of the portion of the

plot just beyond the lower bound, a second major change in slope can be pinpointed - this point serves as the upper bound of the critical LTB load range. This technique could be applied to most of the collected experimental data, although some variations in the trend did exist. A distinct percent change in slope which would indicate the exact location of the lower and upper bounds of the critical LTB load range has not been quantified. 


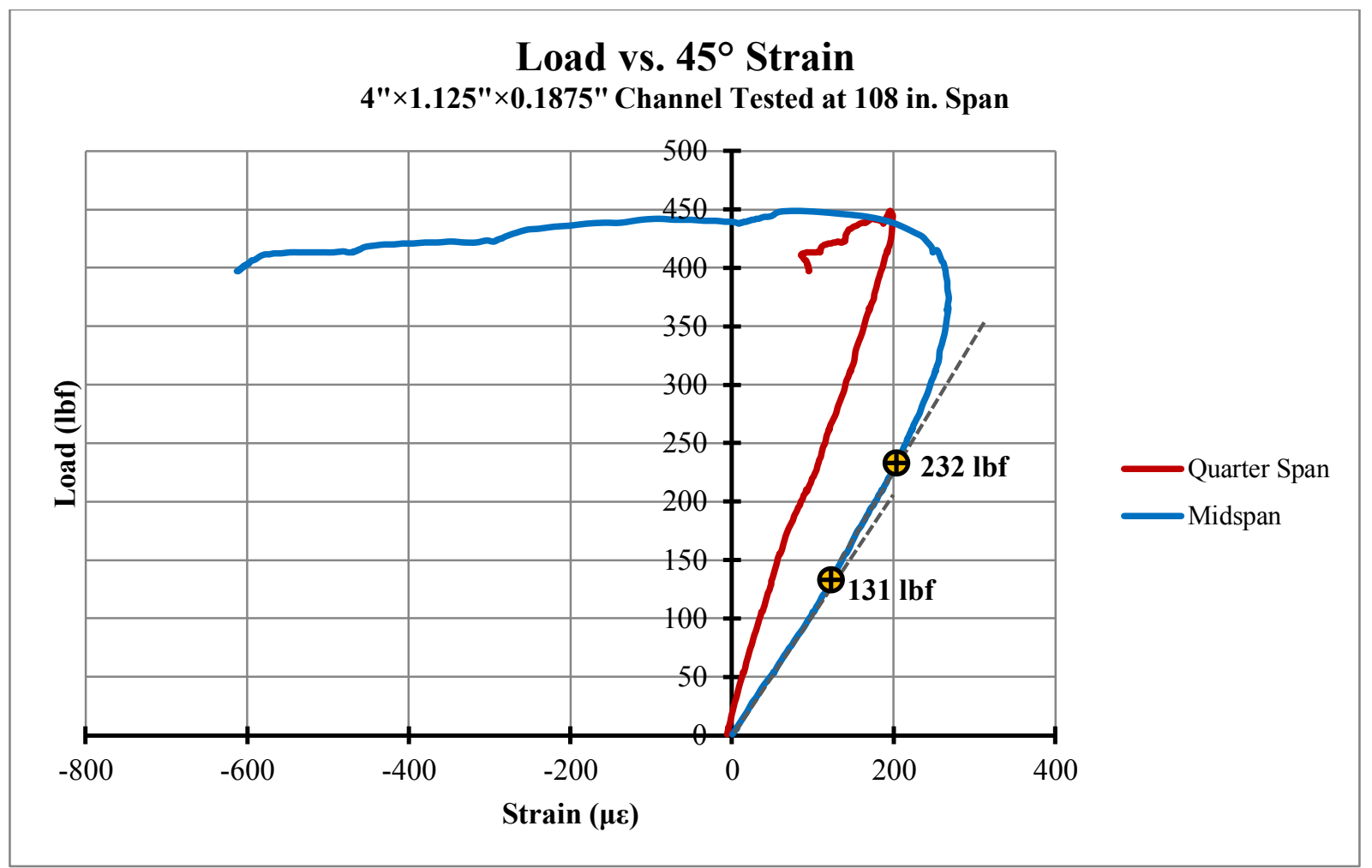

(a)

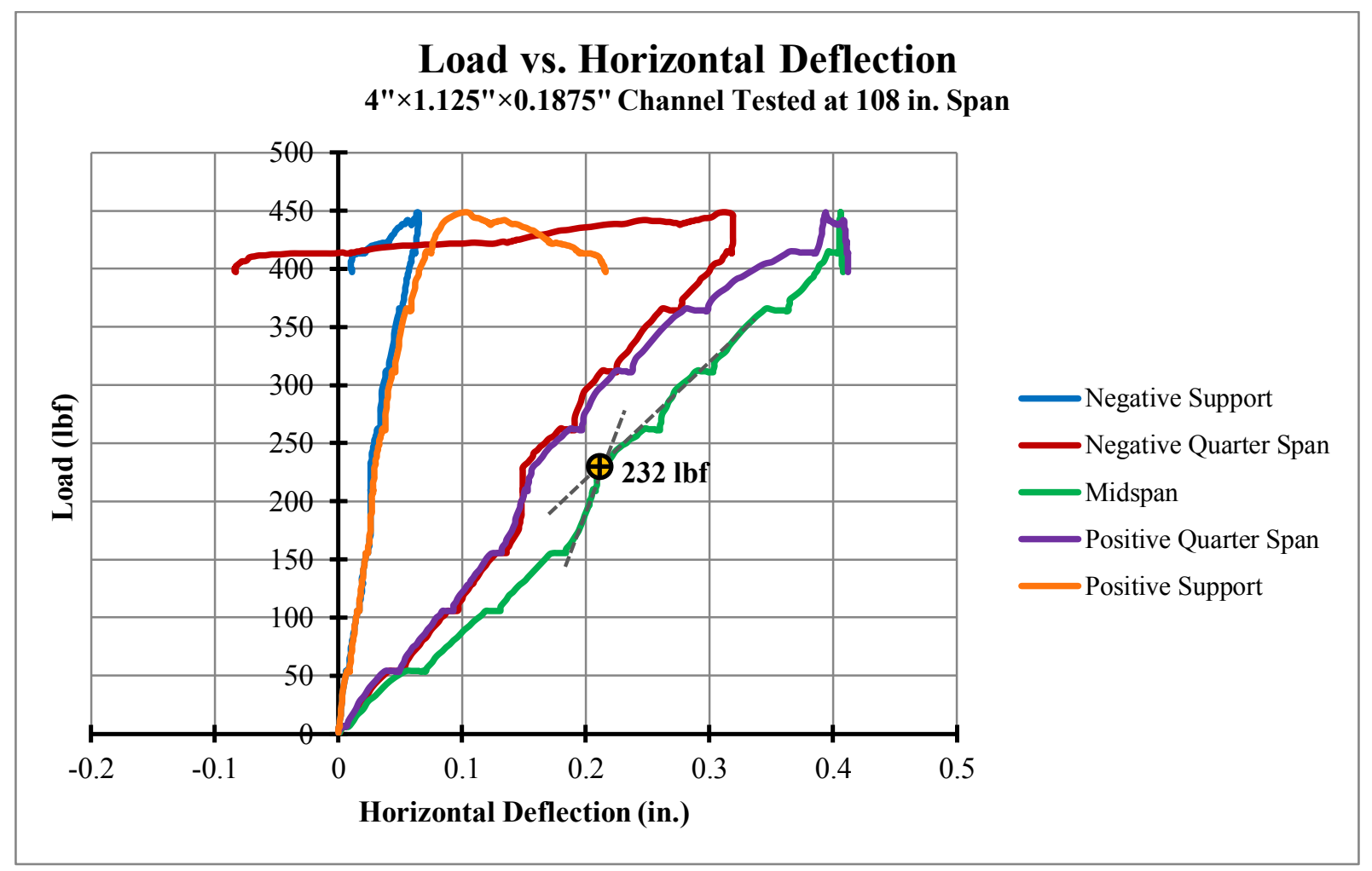

(b)

Figure D-1 (a) Load vs. $45^{\circ}$ Strain plot and (b) Load vs. Horizontal Deflection plot for $4 " \times 1.125^{\prime \prime} \times 0.1875^{\prime \prime}$ Channel Tested at 108 in. Span 


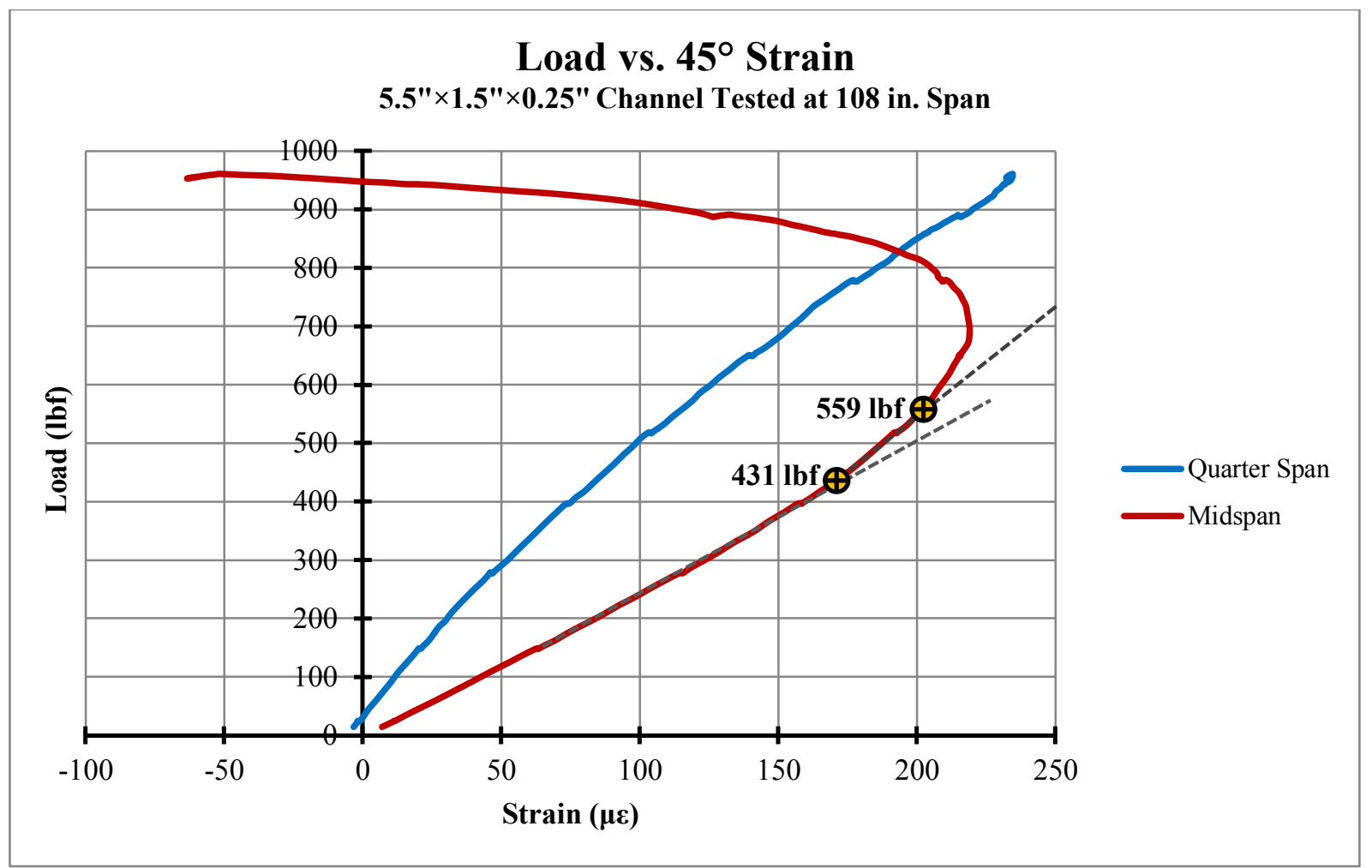

(a)

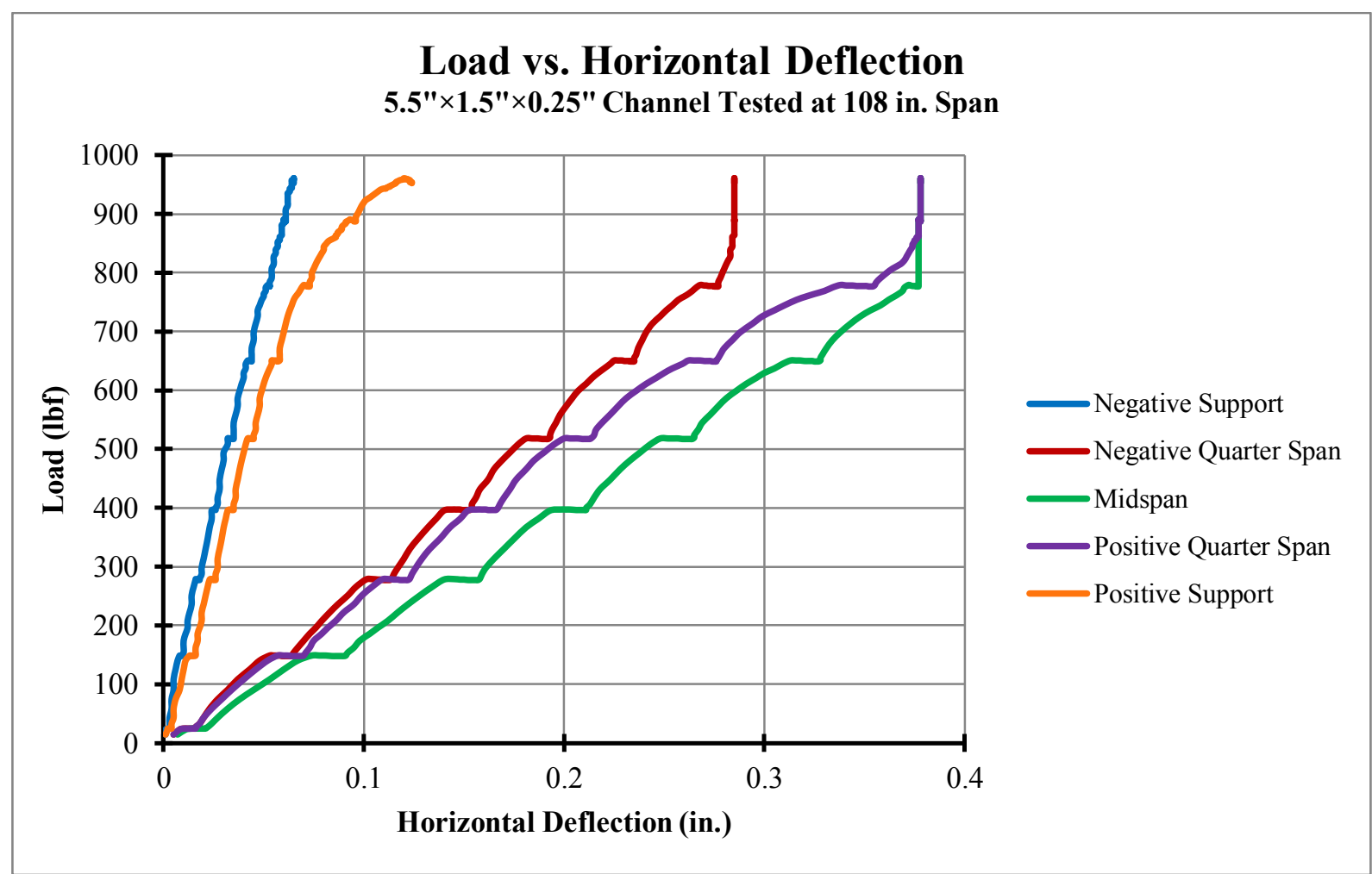

(b)

Figure D-2 (a) Load vs. $45^{\circ}$ Strain plot and (b) Load vs. Horizontal Deflection plot for $5 " \times 1.5 " \times 0.25 "$ Channel Tested at 108 in. Span 


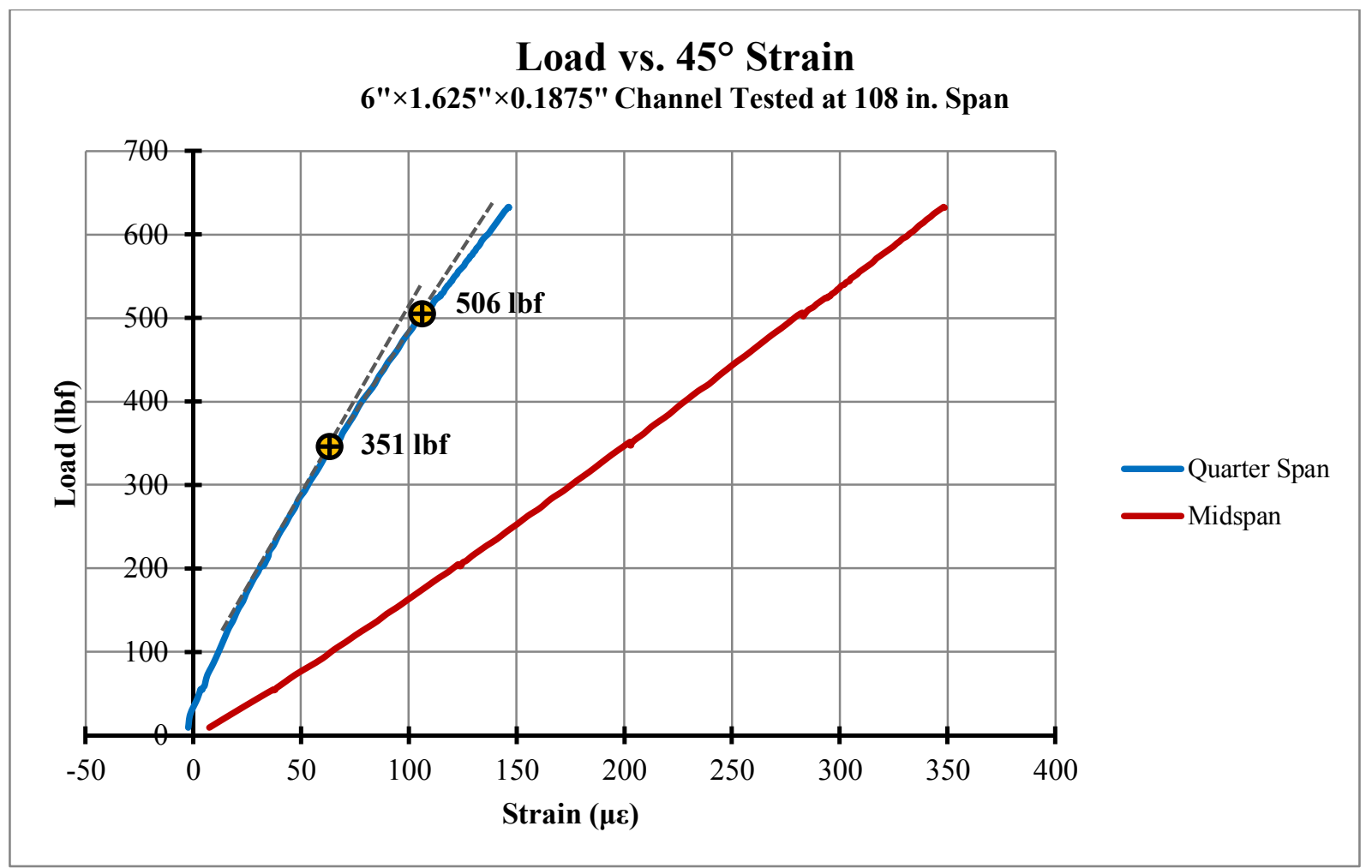

(a)

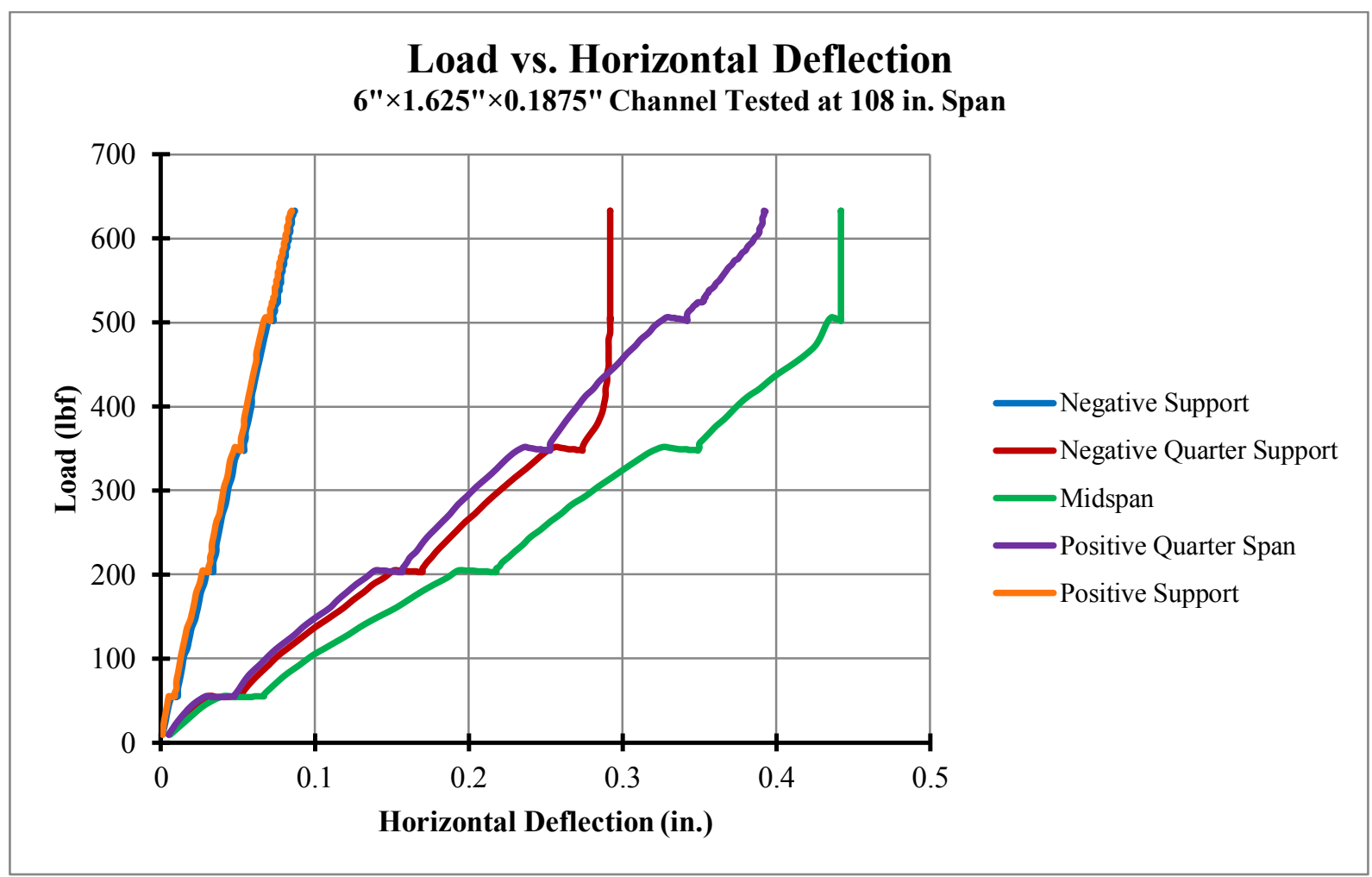

(b)

Figure D-3 (a) Load vs. $45^{\circ}$ Strain plot and (b) Load vs. Horizontal Deflection plot for $6 " \times 1.625^{\prime \prime} \times 0.1875^{\prime \prime}$ Channel Tested at 108 in. Span 


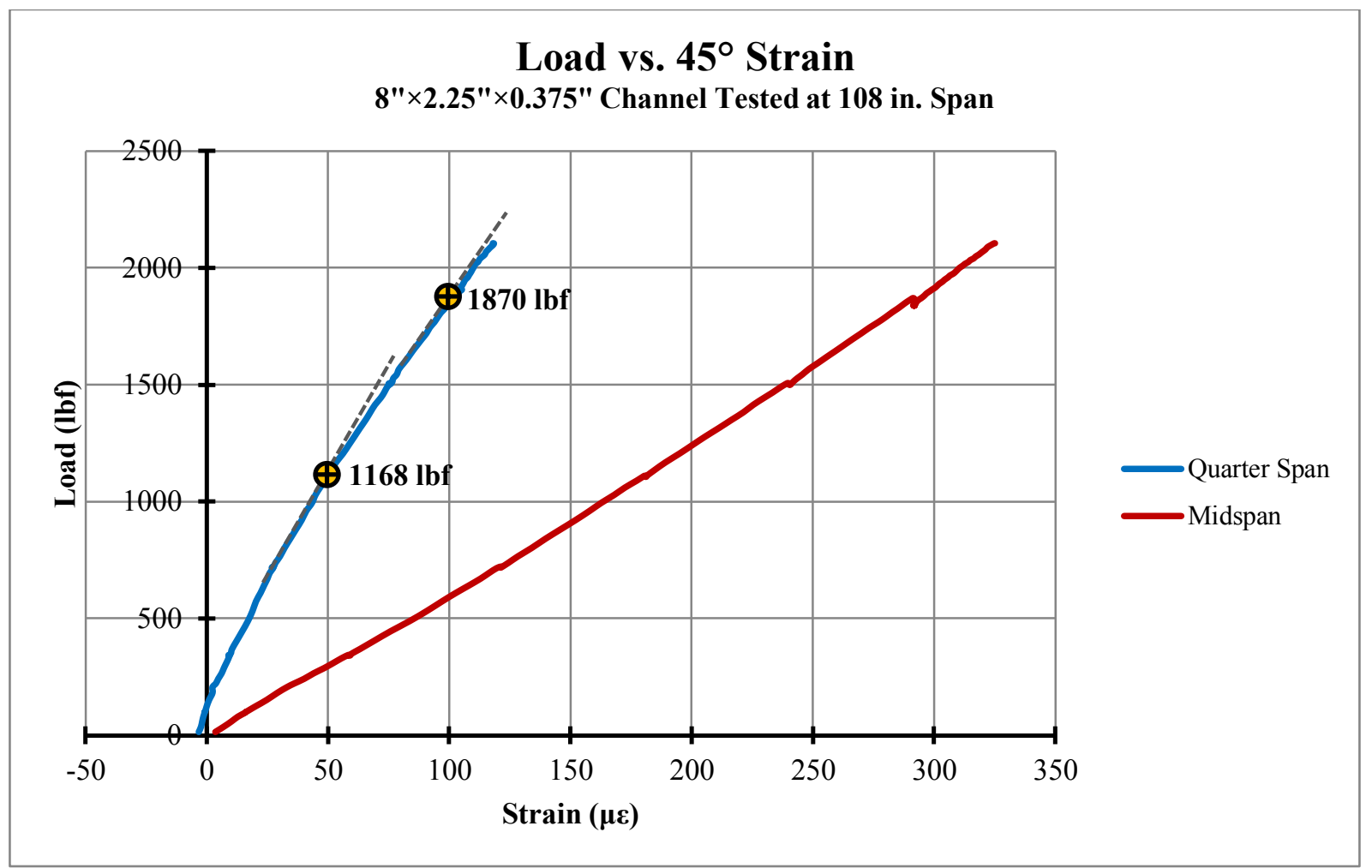

(a)

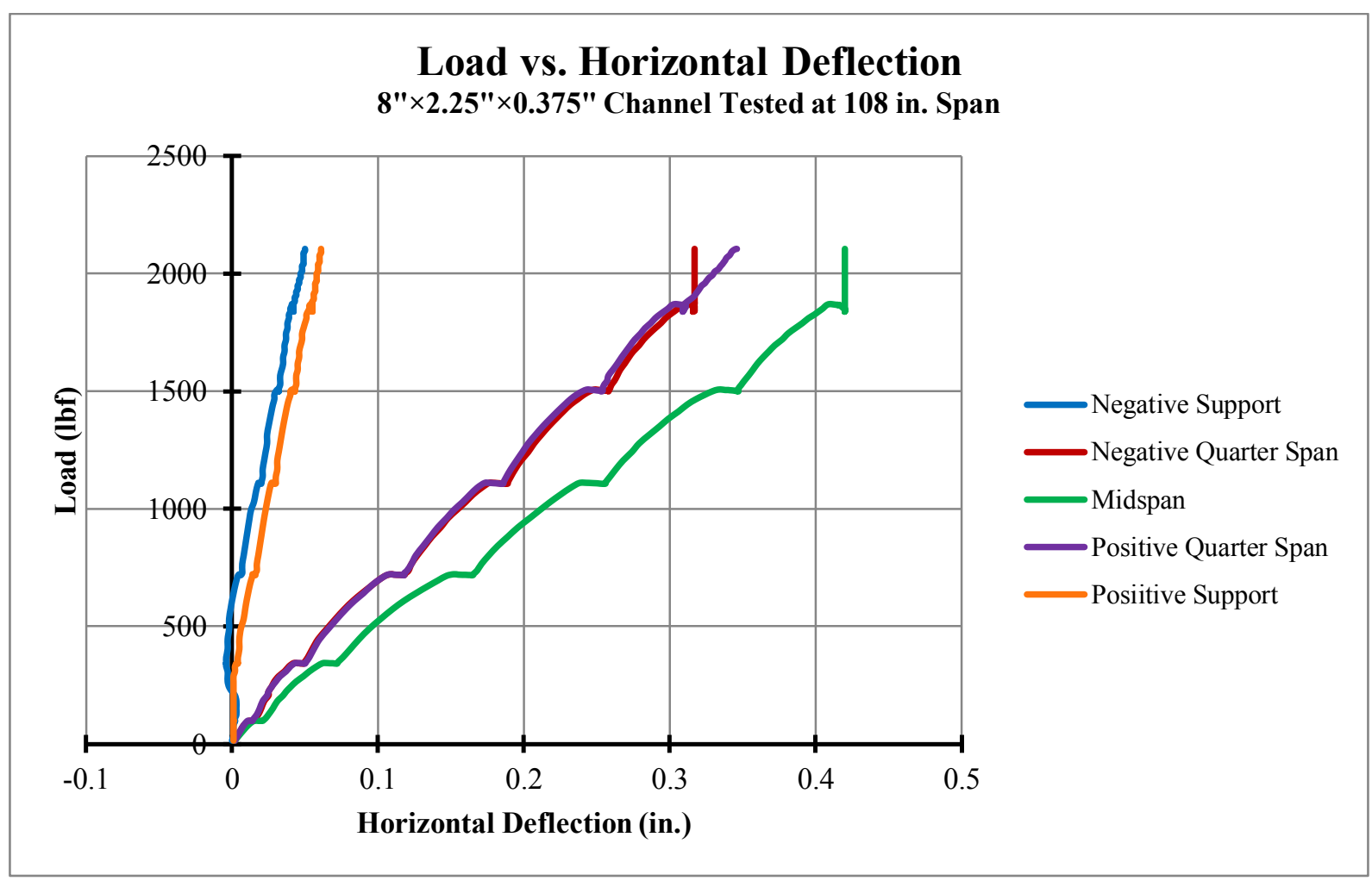

(b)

Figure D-4 (a) Load vs. $45^{\circ}$ Strain plot and (b) Load vs. Horizontal Deflection plot for 8" $\times 2.25 " \times 0.375 "$ Channel Tested at 108 in. Span 


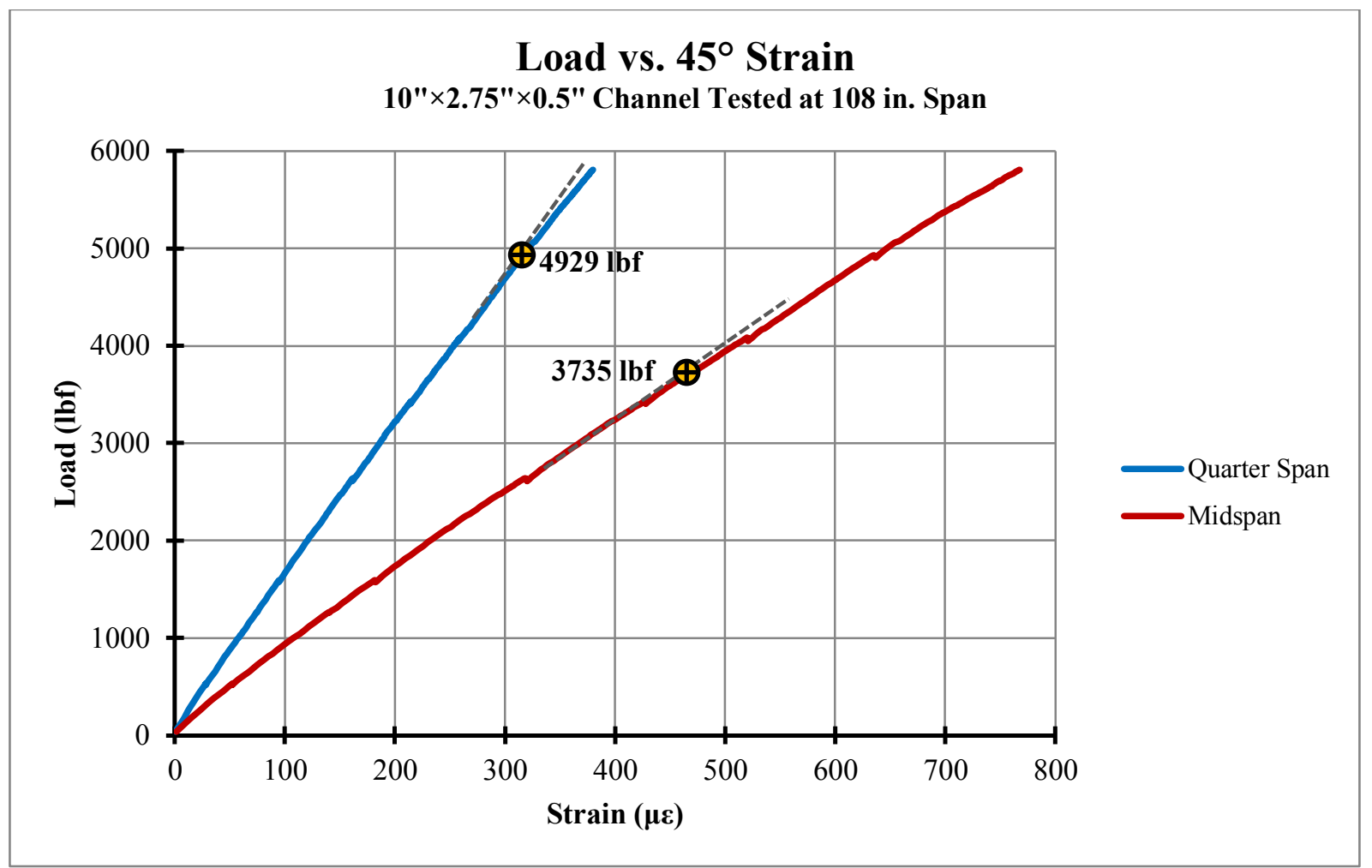

(a)

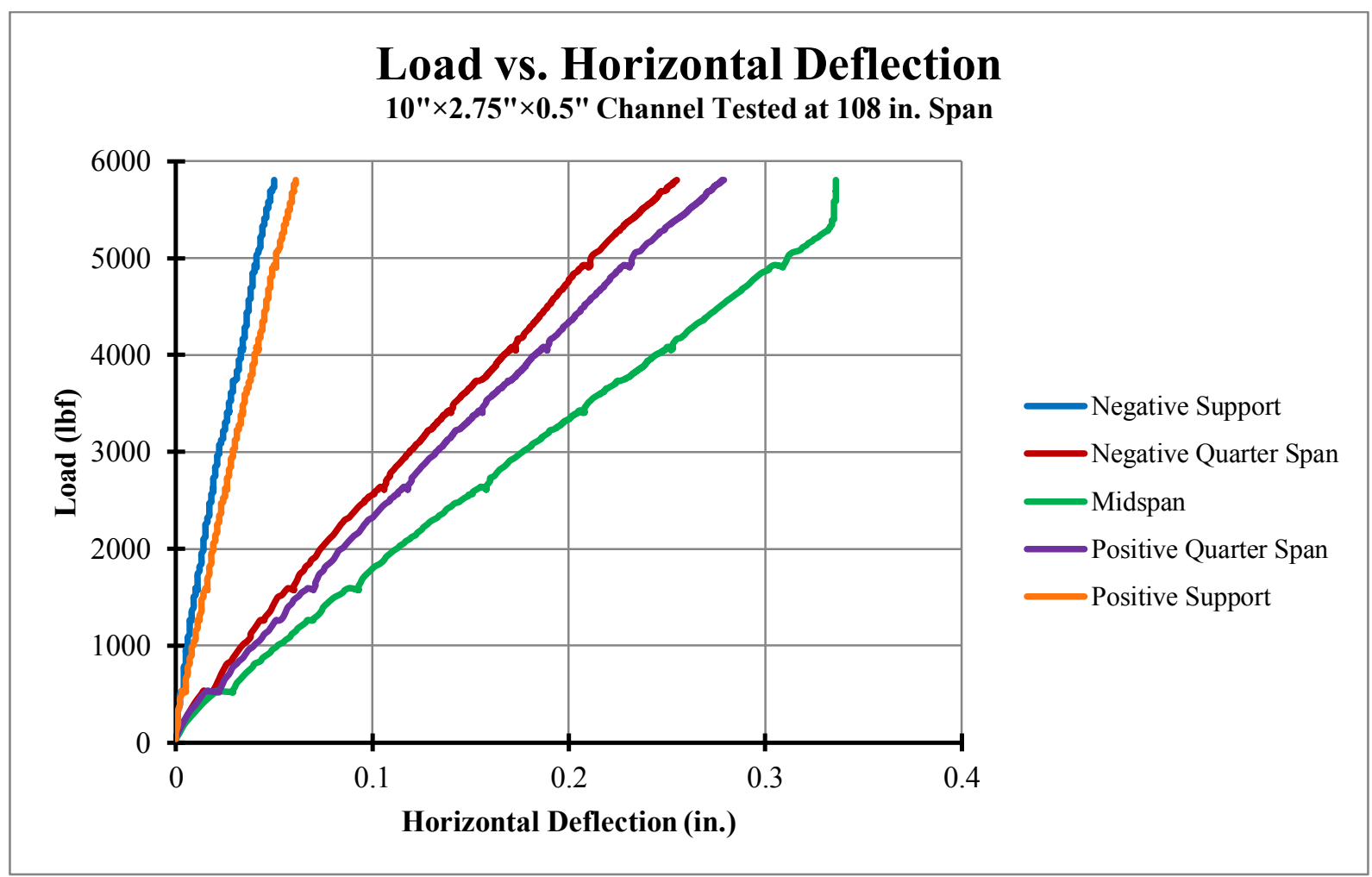

(b)

Figure D-5 (a) Load vs. $45^{\circ}$ Strain plot and (b) Load vs. Horizontal Deflection plot for $10 " \times 2.75^{\prime \prime} \times 0.5^{\prime \prime}$ Channel Tested at 108 in. Span 


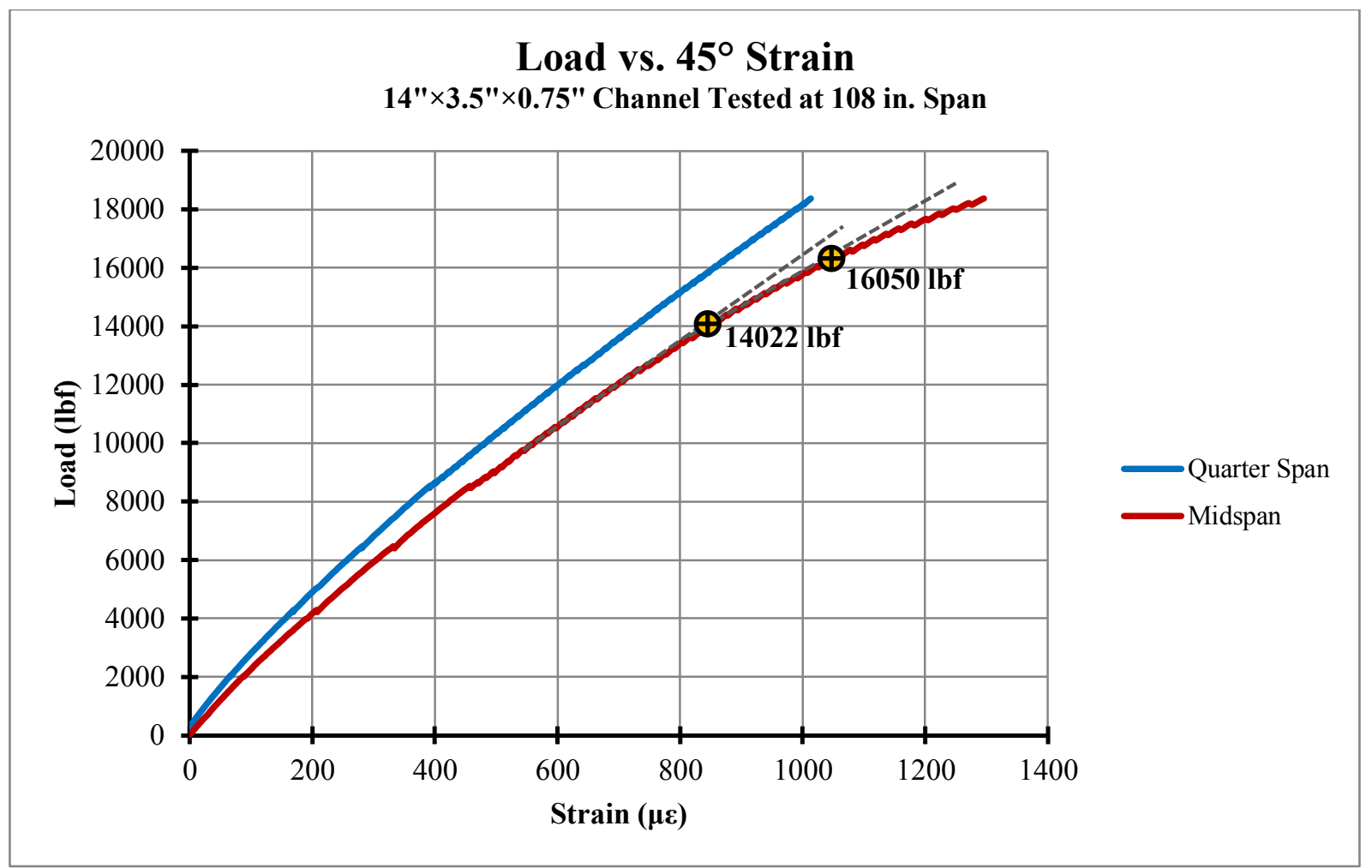

(a)

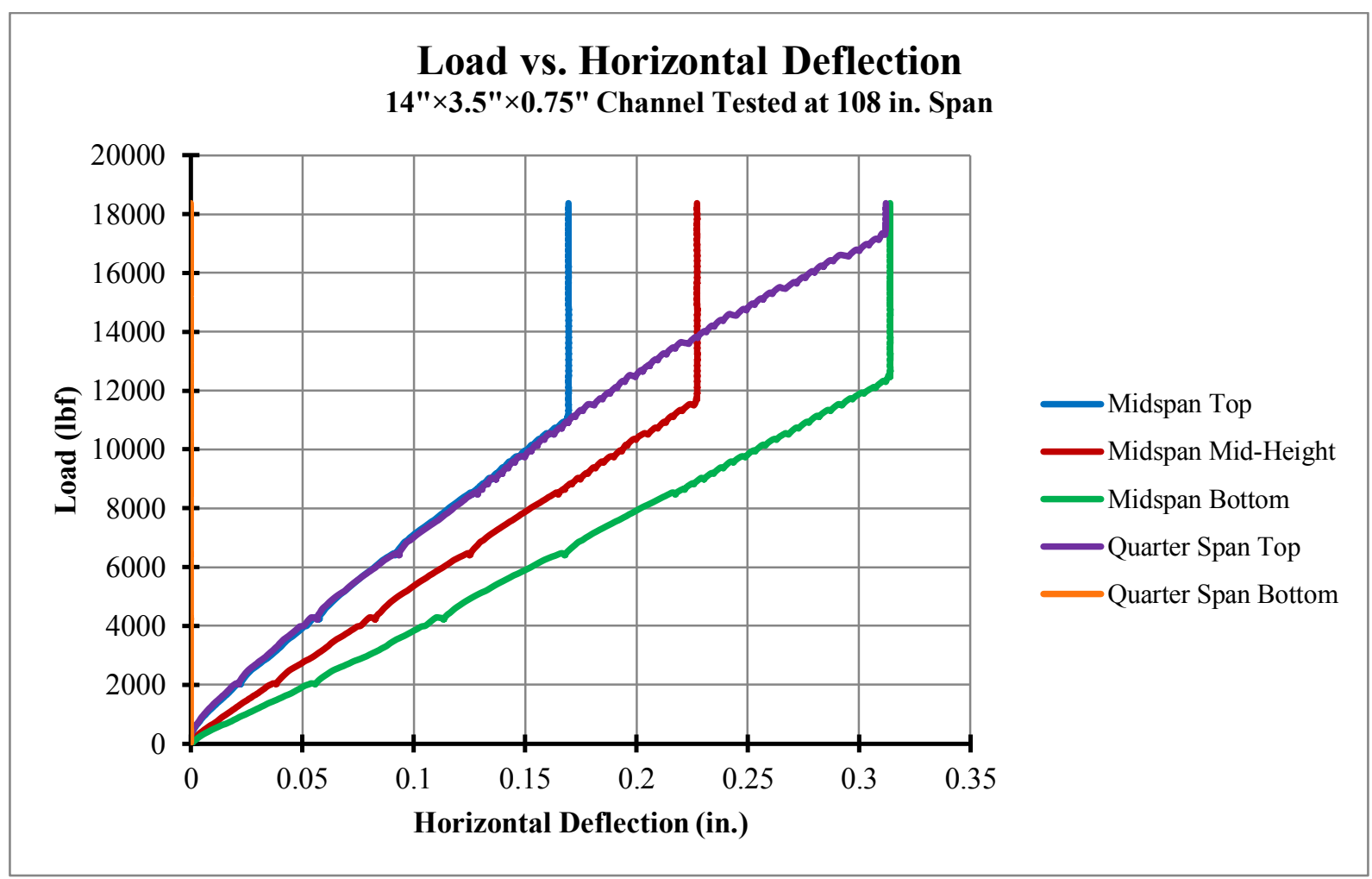

(b)

Figure D-6 (a) Load vs. $45^{\circ}$ Strain plot and (b) Load vs. Horizontal Deflection plot for $14 " \times 3.5^{\prime \prime} \times 0.75 "$ Channel Tested at 108 in. Span 


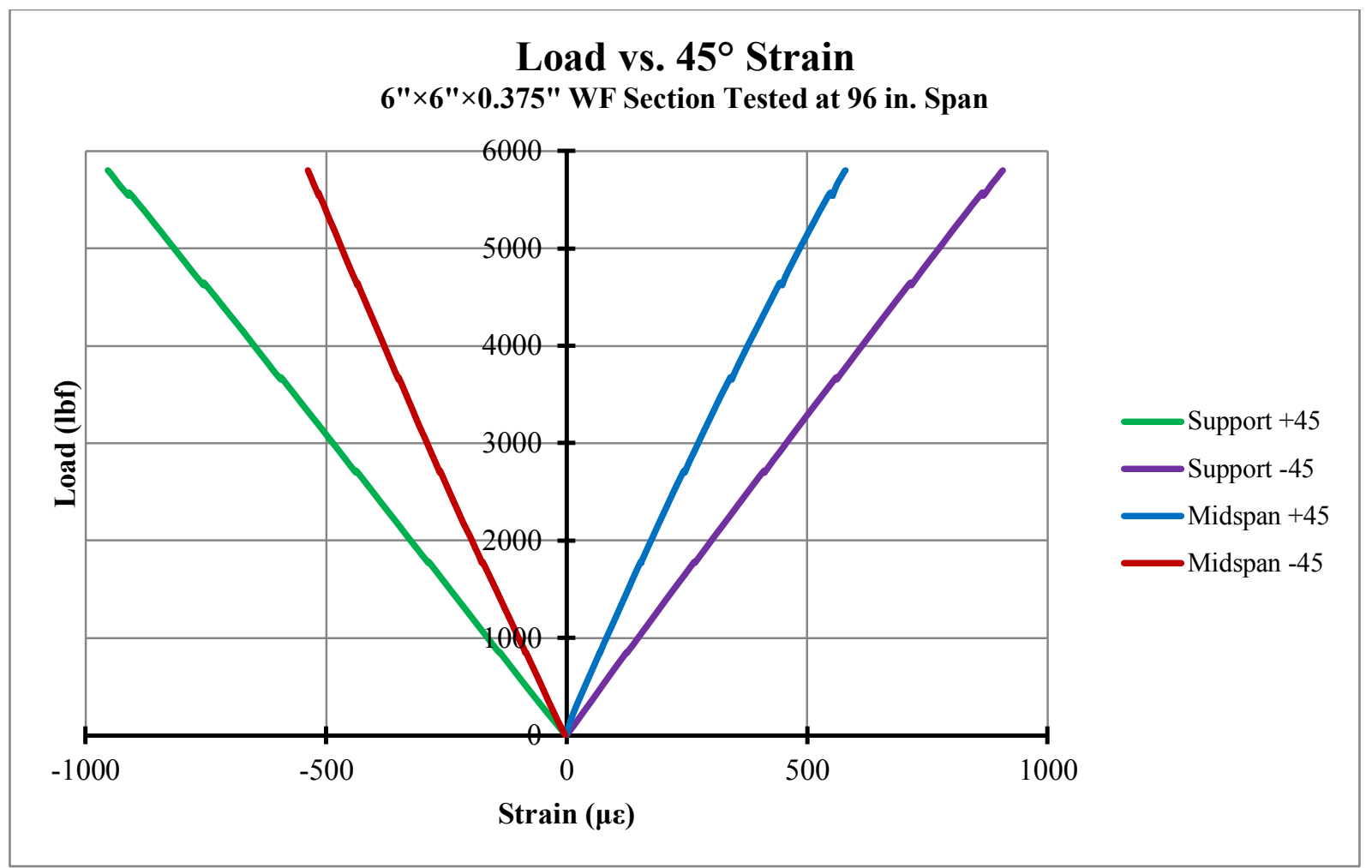

(a)

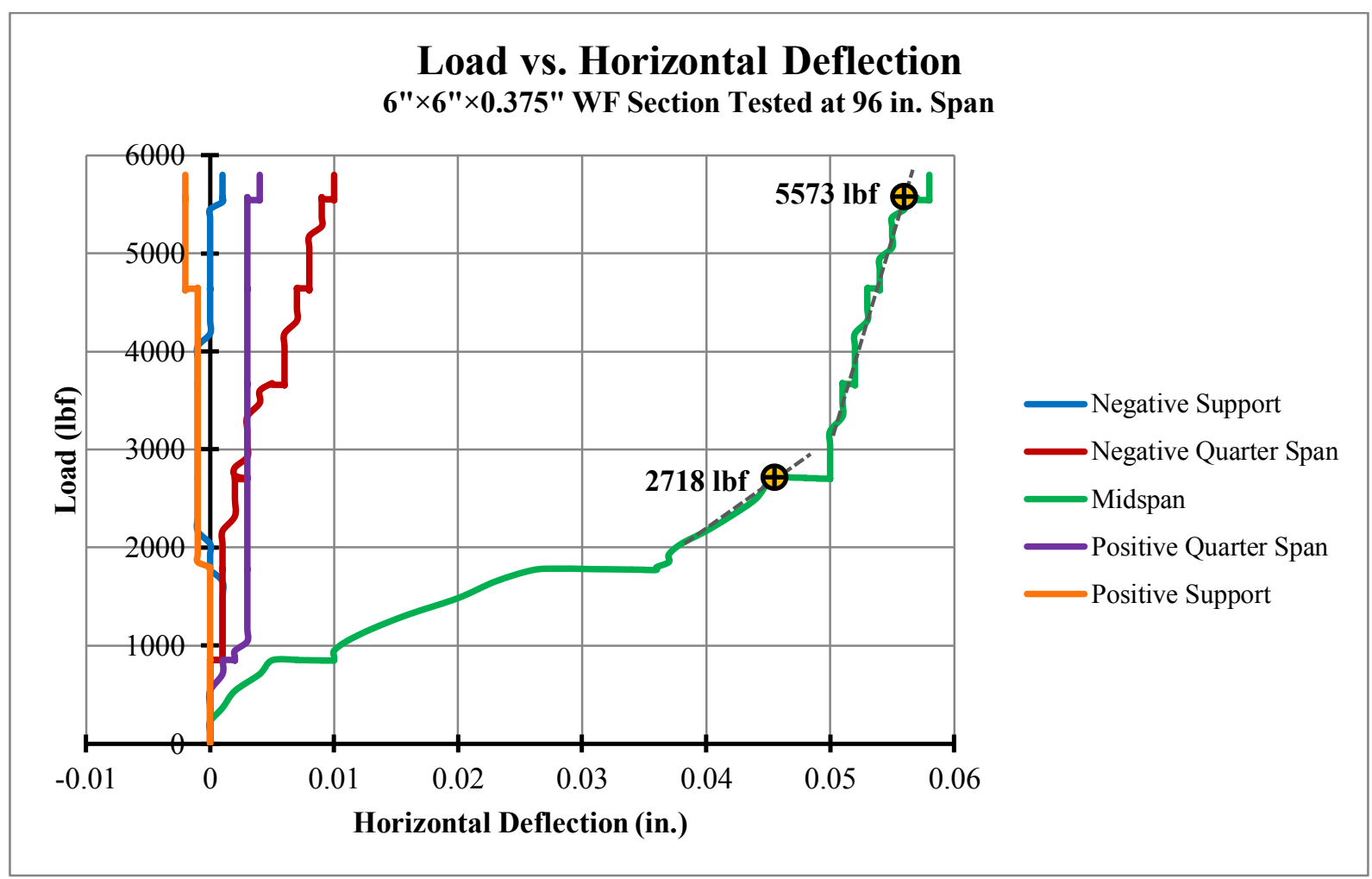

(b)

Figure D-7 (a) Load vs. $45^{\circ}$ Strain plot and (b) Load vs. Horizontal Deflection plot for $6 " \times 6 " \times 0.375 "$ WF Section Tested at 96 in. Span 


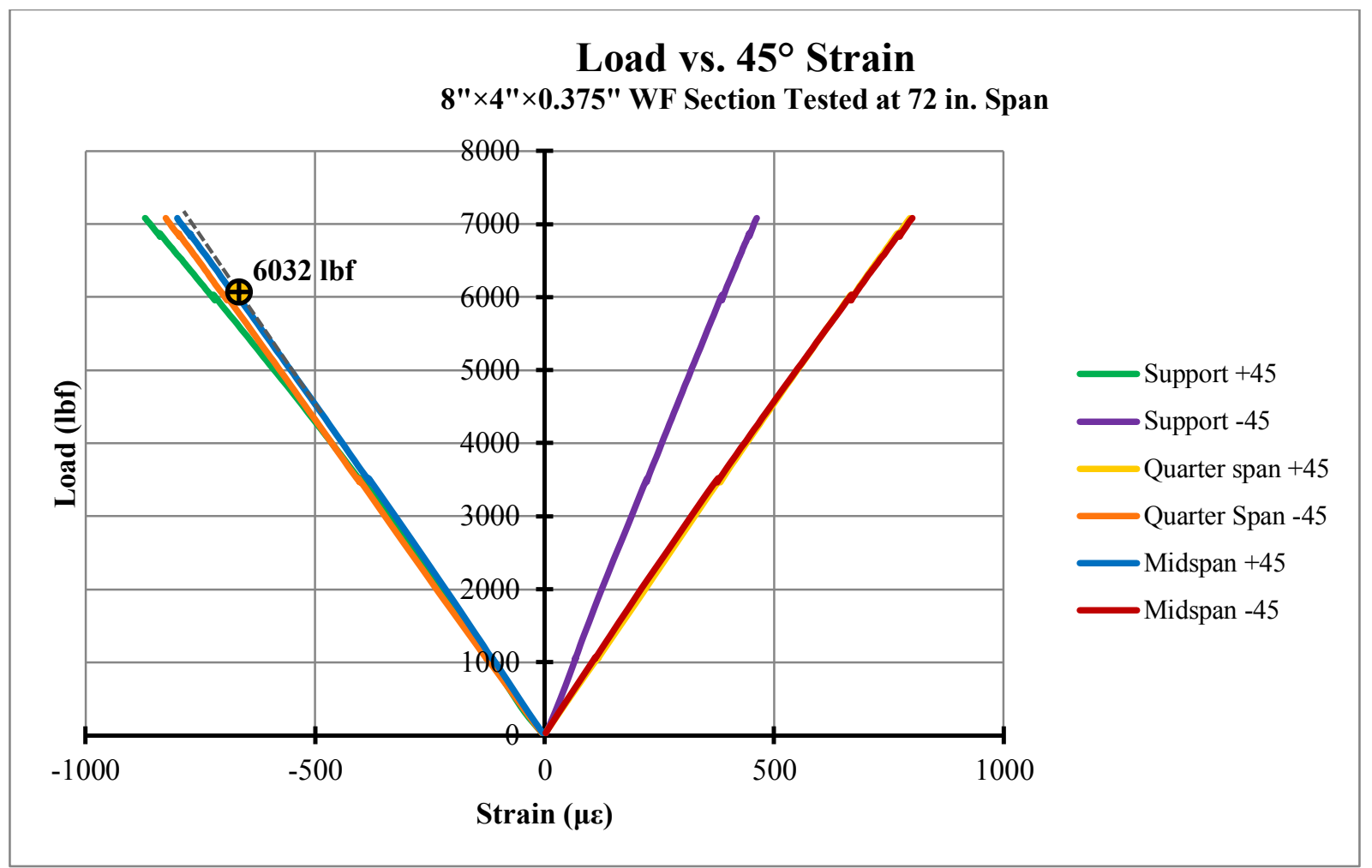

(a)

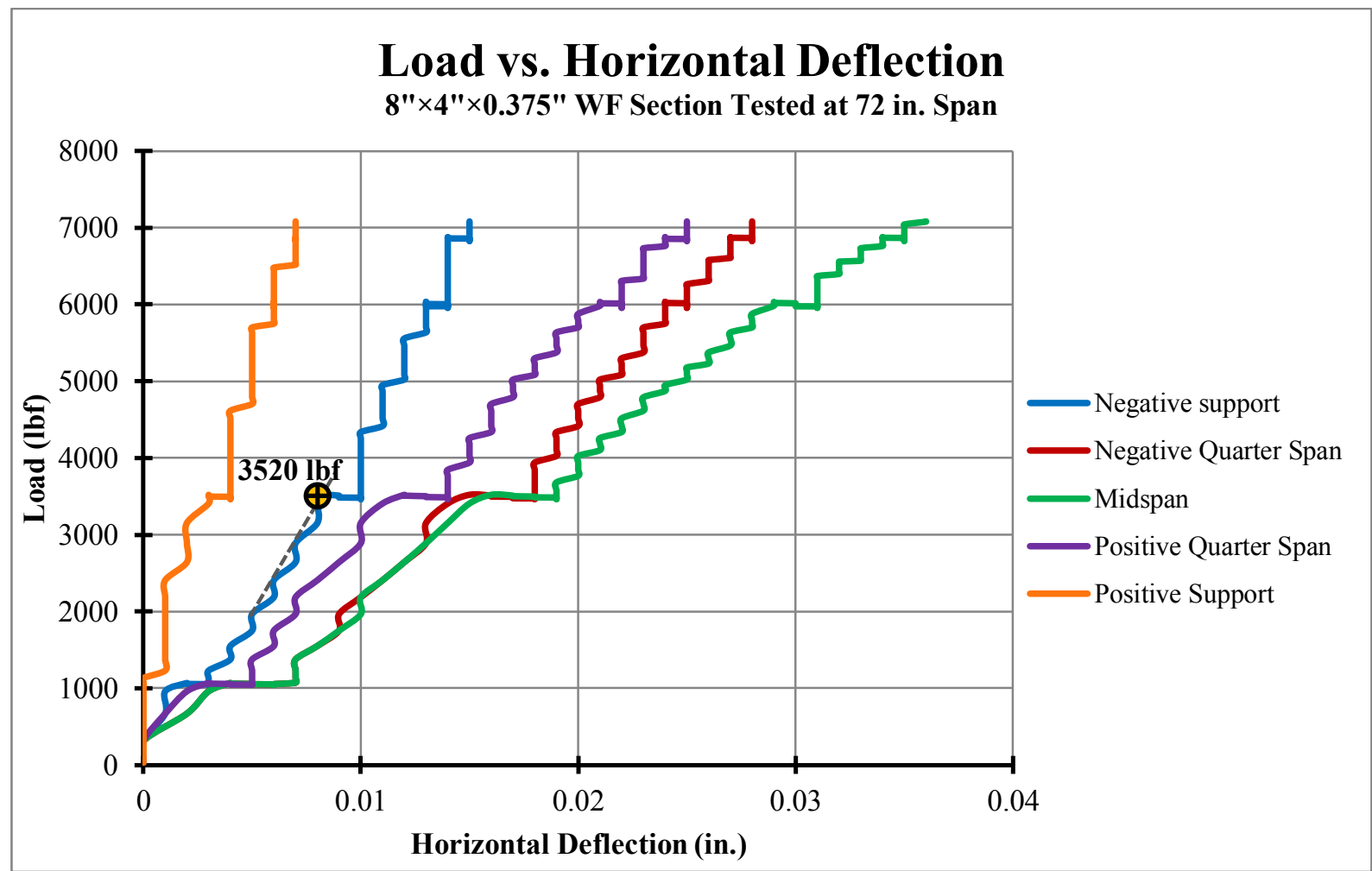

(b)

Figure D-8 (a) Load vs. $45^{\circ}$ Strain plot and (b) Load vs. Horizontal Deflection plot for 8" $\times 4 " \times 0.375^{\prime \prime}$ WF Section Tested at 72 in. Span 


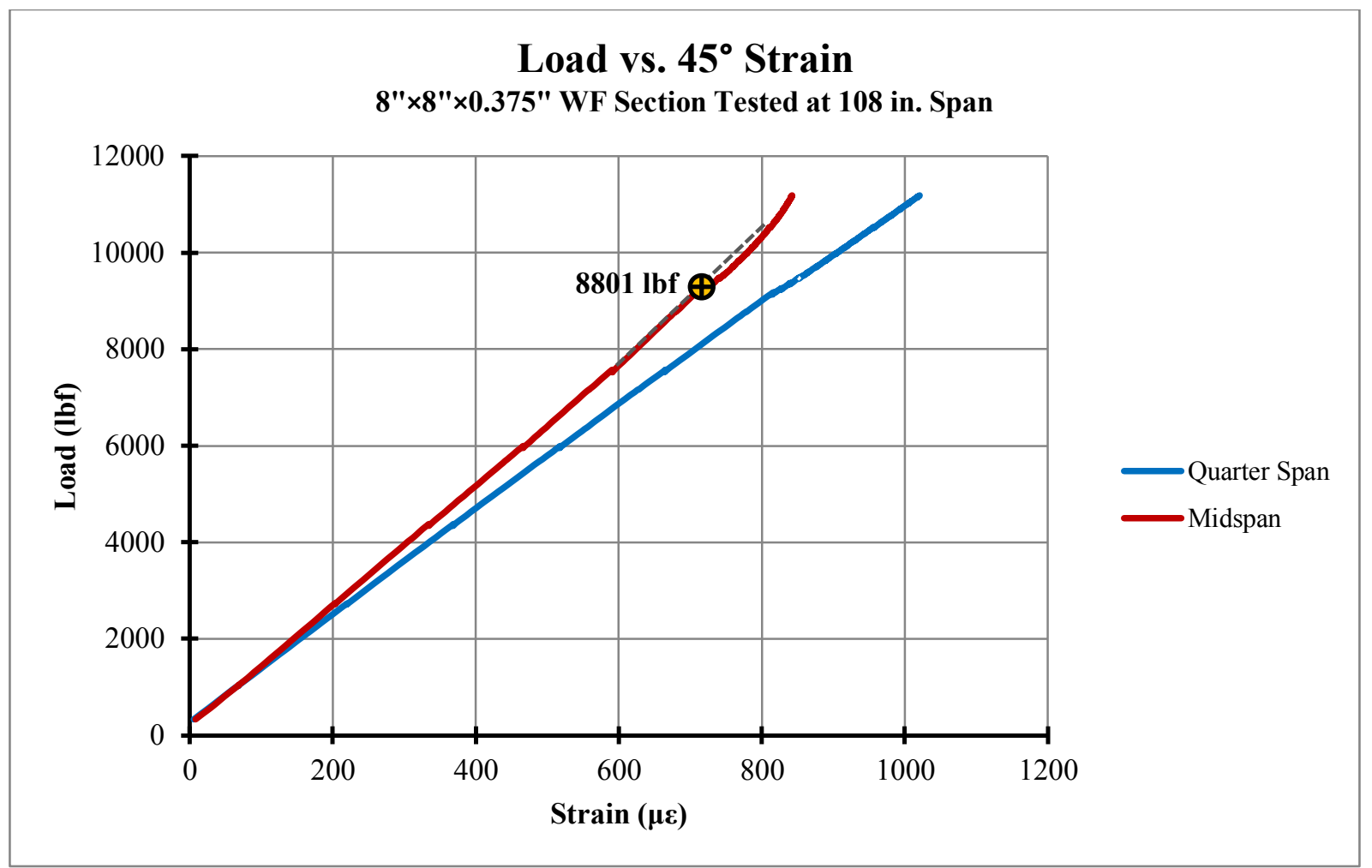

(a)

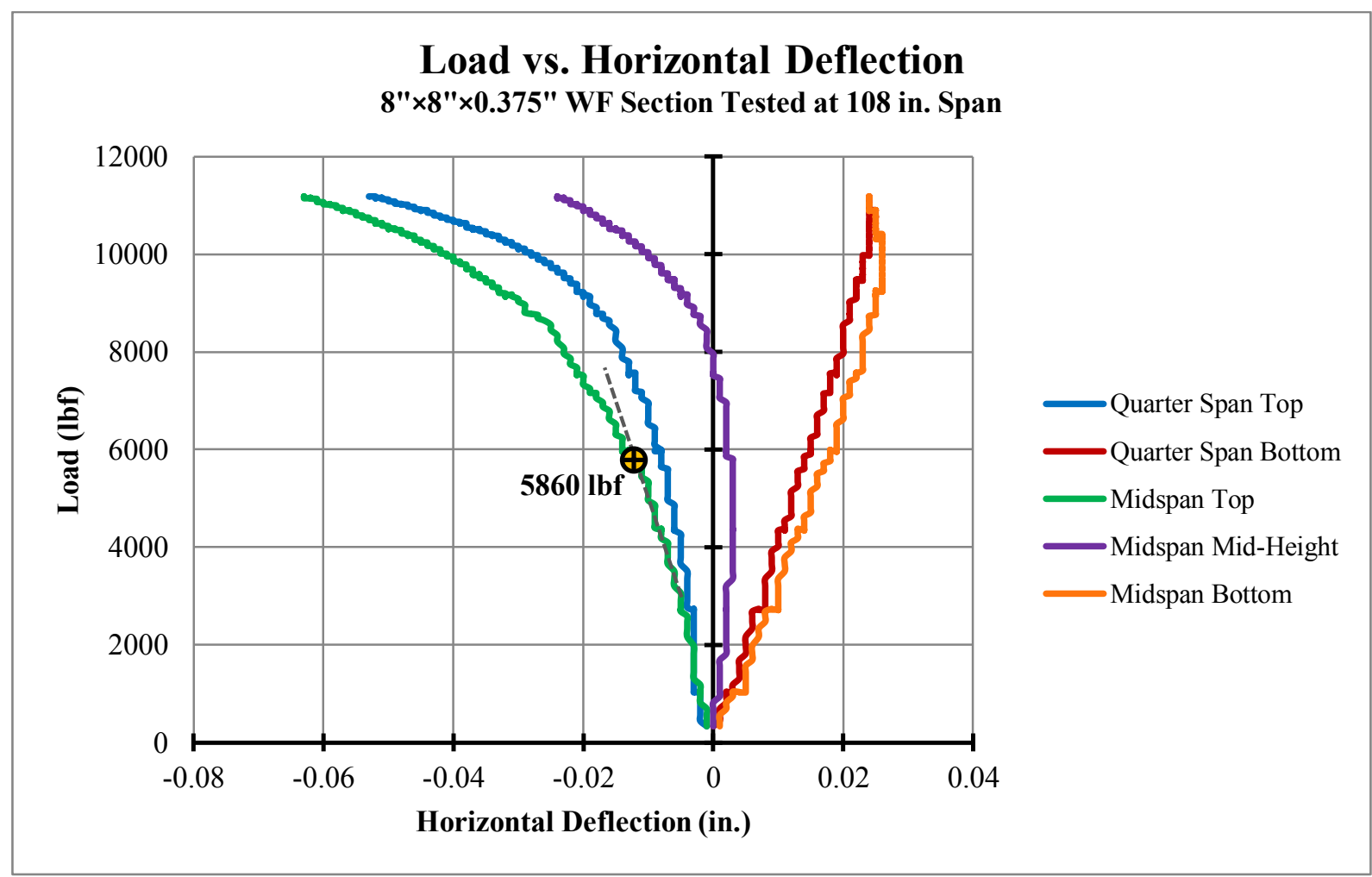

(b)

Figure D-9 (a) Load vs. $45^{\circ}$ Strain plot and (b) Load vs. Horizontal Deflection plot for 8" $\times 8 " \times 0.375^{\prime \prime}$ WF Section Tested at 108 in. Span 


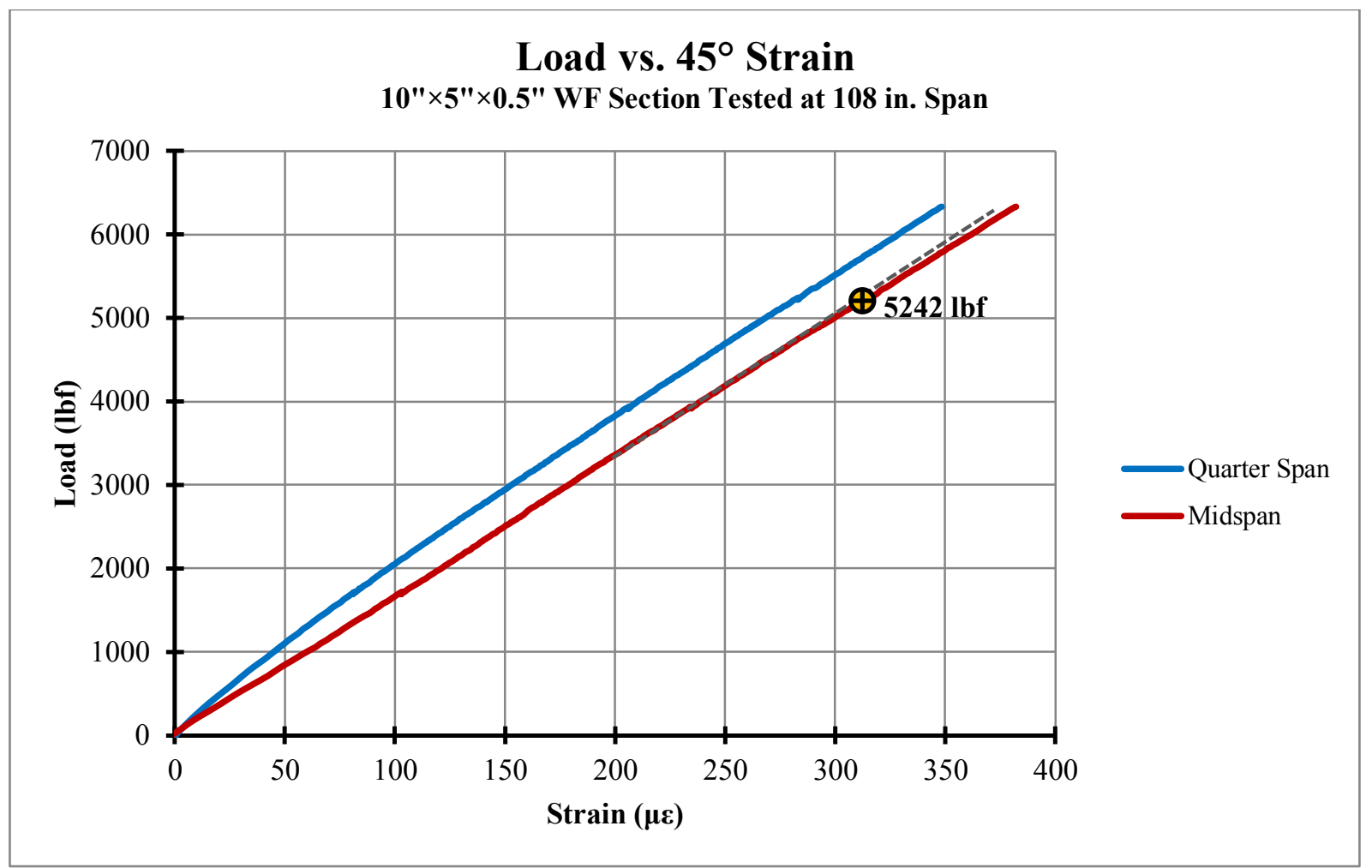

(a)

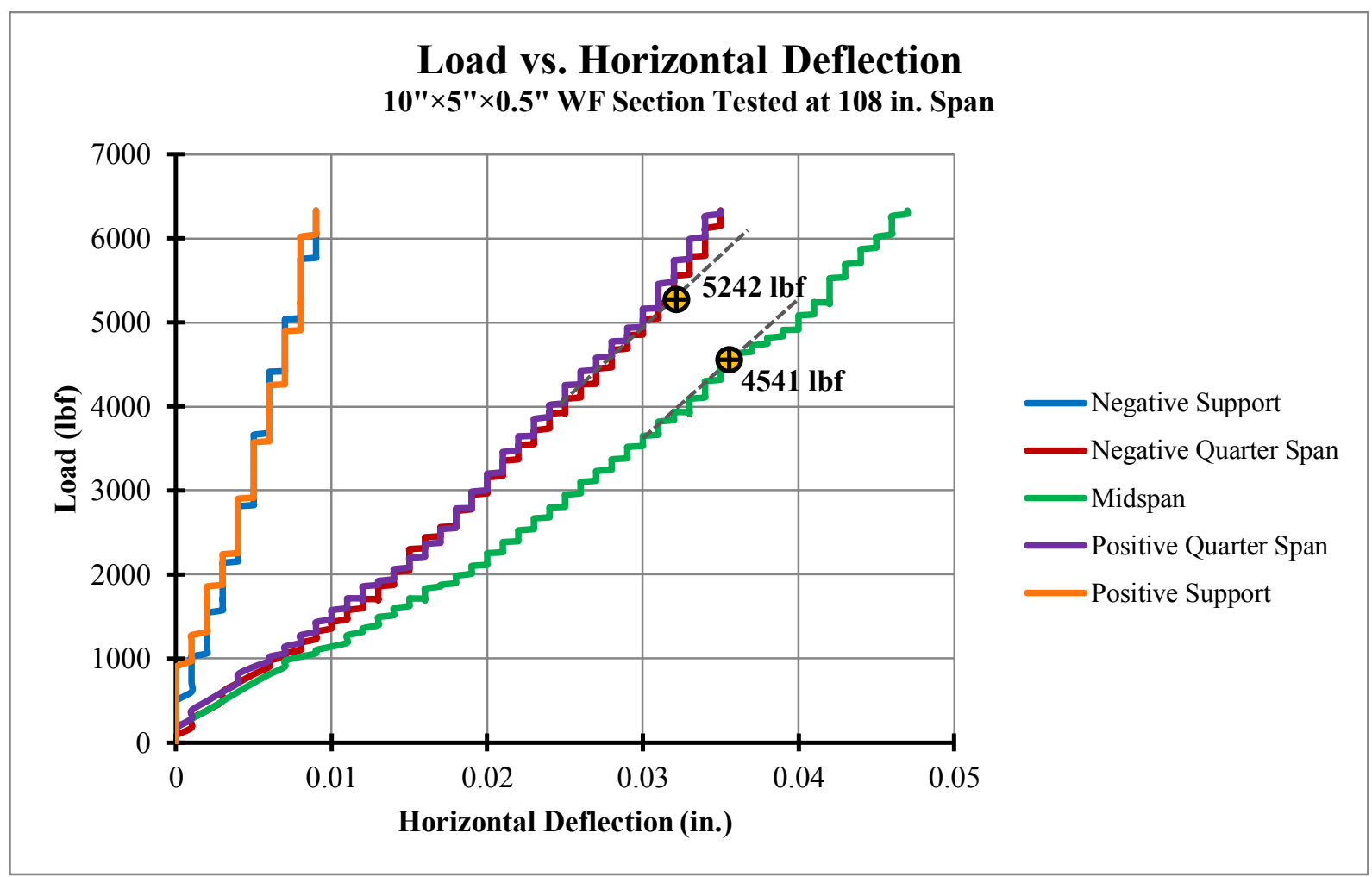

(b)

Figure D-10 (a) Load vs. $45^{\circ}$ Strain plot and (b) Load vs. Horizontal Deflection plot for $6 " \times 6 " \times 0.375^{\prime \prime}$ WF Section Tested at 96 in. Span 


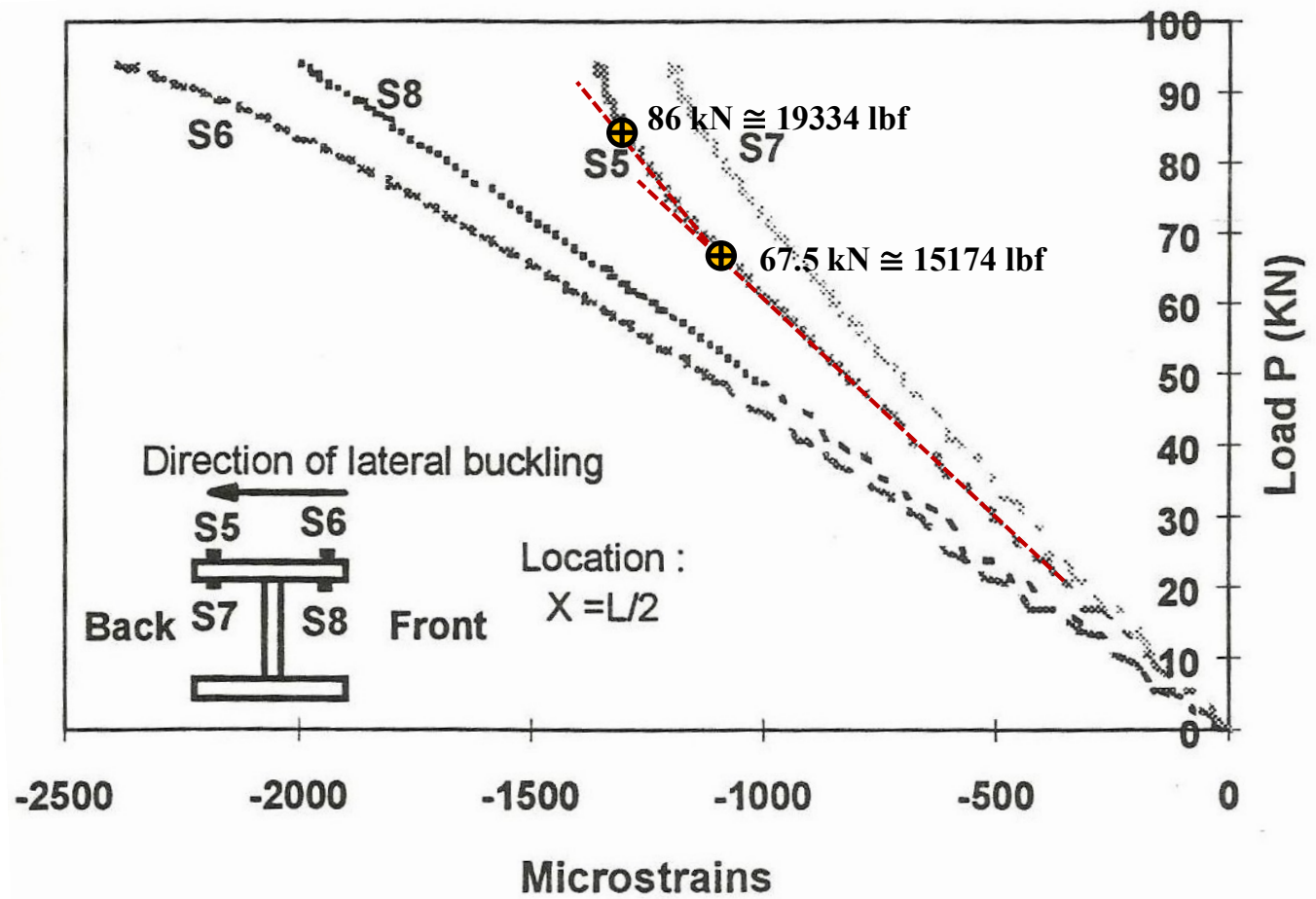

Figure D-11 Load vs. Strain in the Compression Flange for $12 " \times 12 " \times 0.5 "$ WF Section Tested Under Four-point Bending at 180 in. Span by Bendidi (1996) 